\title{
Low Temperature Phase of the $m$-component Spin Glass
}

\author{
Dissertation \\ zur Erlangung des Doktorgrades \\ der Mathematisch-Naturwissenschaftlichen Fakultäten \\ der Georg-August-Universität Göttingen
}

vorgelegt von

Axel Braun

aus Kassel

Göttingen, 2011 
D7

Referent: Prof. Dr. Annette Zippelius Korreferent: PD Dr. Timo Aspelmeier Tag der mündlichen Prüfung: 


\section{Contents}

$\begin{array}{lll}1 & \text { Introduction } & 1\end{array}$

1.1 The Sherrington-Kirkpatrick Ising spin glass . . . . . . . . . . 4

1.2 Return to Introduction . . . . . . . . . . . . . . . . . . . 11

1.3 State of the Art . . . . . . . . . . . . . . . . . . . 14

1.4 Motivation . . . . . . . . . . . . . . . . . . 18

$\begin{array}{lll}2 & \text { Phase Transition } & 21\end{array}$

2.1 Cavity method and effective-field approach . . . . . . . . . . . 22

2.2 Finite component numbers . . . . . . . . . . . . . . . . 33

2.3 Configurations of effective fields . . . . . . . . . . . . . 35

2.4 Parallel Tempering Monte Carlo algorithm . . . . . . . . . . . 42

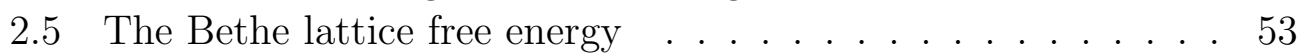

\begin{tabular}{|lll}
\hline & Generalized Bose-Einstein condensation & 61
\end{tabular}

$3.1 \quad$ Effective Component Number at $T=0$. . . . . . . . . . . . . 62

3.2 Effective Component Number for finite $T$. . . . . . . . . . . . 66

\begin{tabular}{|lll}
4 & Breaking Replica Symmetry & 81
\end{tabular}

4.1 Self-consistent effective field approach . . . . . . . . . . . . . . 82

4.2 Parallel Tempering . . . . . . . . . . . . . . . . . . . . . . . 87

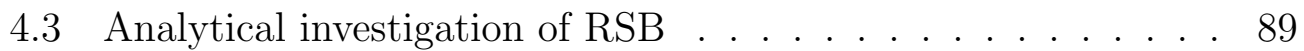

5 Fluctuations of the Free Energy 115

5.1 Connection between Fluctuations and Bond Chaos . . . . . . 119

$5.2 \quad$ Calculating $P_{\epsilon}\left(q_{L}\right) \quad \ldots \ldots \ldots \ldots \ldots \ldots$

5.3 Solving the Saddle Point Equations . . . . . . . . . . . . . . 127

5.4 Calculate the sample-to-sample Fluctuations . . . . . . . . . . 137

\begin{tabular}{lll}
\hline 6 & Conclusion & 143
\end{tabular}

\section{Appendices}


\begin{tabular}{|ll}
\hline A Effective field approach & 149
\end{tabular}

\begin{tabular}{llr}
\hline B & Appendix to Section 5.3 & 153
\end{tabular}

B.1 Additional expression for $g_{n}^{k}(\vec{\sigma})$. . . . . . . . . . . . . . 153

B.2 Convenient form of the free energy . . . . . . . . . . . . . . . 154

B.3 $\quad$ The free energy in terms of the overlaps . . . . . . . . . . . . 156

B.4 Calculating $q_{\alpha \beta}^{\mu \nu(0)}$ in spherical coordinates . . . . . . . . . . . 160

B.5 Wick's theorem . . . . . . . . . . . . . . . . 163

B.6 Diagonalisation and inverse of $M_{0} \ldots \ldots$. . . . . . . . . 165

B.7 $\quad$ Maximizing the free energies with respect to $r \ldots . . .168$

\begin{tabular}{ll}
\hline C Introducing bond chaos & 171
\end{tabular}

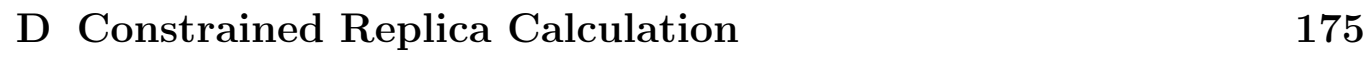

\begin{tabular}{lc}
\hline List of Figures & 183
\end{tabular}

\begin{tabular}{ll}
\hline List of Tables & 185
\end{tabular}

\begin{tabular}{lr}
\hline Bibliography & 185
\end{tabular} 


\section{Chapter 1}

\section{Introduction}

Spin glasses are a fascinating topic in statistical physics ever since the first attempts to describe them analytically by Edwards and Anderson [1] and Sherrington and Kirkpatrick [2] in 1975. Starting by trying to understand experimentally observed properties of a special class of magnetic alloys, see Ref. [3], and summarizing the characteristic features of systems that exhibit such properties under the name of spin glasses, they soon became a challenging field in theoretical physics, see Ref. [4, 5]. The most prominent phenomenon of spin glasses is a transition into a phase where the spins become frozen in time, following the phase transition of a ferromagnet. Such a freezing of the spins is expected to happen in a disordered way, in contrast to the uniform freezing of spins in a ferromagnet.

A standard example of such a magnetic alloy is the diluted solution of manganese $(\mathrm{Mn})$ in copper $(\mathrm{Cu})$. The magnetic $\mathrm{Mn}$ atoms occupy random positions in a non-magnetic matrix of $\mathrm{Cu}$ atoms. Therefore the distance between them is random as well. Each atom carries a magnetic moment, the spin, which can be simply understood as a vector. Generally, two atoms (or ions, etc.) with certain magnetic moments interact magnetically through an exchange interaction between them, which is a scalar described by the RKKY potential (named after Ruderman, Kittel, Kasuya, Yosida) in case of a metal. This potential oscillates with respect to the distance between both particles. Due to the random positions of the Mn atoms, the interaction between each pair of them can be ferromagnetic (positive) or antiferromagnetic (negative), preferring parallel or antiparallel alignment of the spins concerned. This special disorder due to the mixture of ferromagnetic and antiferromagnetic bonds is characteristic of all members of the class of spin glasses and can have different causes (the RKKY potential between spins is not necessary in general). The magnetic properties of such systems are solely determined by the configuration of the spins of these atoms, therefore a state in thermal 
equilibrium of a spin glass is the result of these competing ferromagnetic and antiferromagnetic exchange interactions, which we call a bond or a link in the following. In theoretical physics, such a bond between spin $s_{i}$ and $s_{j}$ is described as a random variable, denoted by $J_{i j}$, which is distributed according to a probability distribution, which allows for ferromagnetic and antiferromagnetic bonds. In this way one approximates experimentally observed interactions described, for instance, by the RKKY potential. The $J_{i j}$ 's are called quenched variables, as they are expected not to change on experimental time scales (in contrast, thermal fluctuations of the spins do happen on these scales). Modelling a spin glass in that way makes the exact position of a single atom redundant, which would be necessary to know in order to calculate the interactions according to their RKKY potential (which seems impossible). Following from this, the generic Hamiltonian describing a spin glass is remarkably simple compared to the enormous amount of scientific contributions to this field. It is

$$
H=-\frac{1}{2} \sum_{i j} J_{i j} s_{i} s_{j} .
$$

We see that it answers the purpose of lowering the energy of a sample, if two spins $s_{i}$ and $s_{j}$ align parallel for a ferromagnetic bond between them, $J_{i j}>0$, and antiparallel for an antiferromagnetic bond, $J_{i j}<0$. The factor of $\frac{1}{2}$ prevents counting a single link twice. Over the years, various models have emerged, concerning for instance the dimension of the underlying lattice structure, degrees of connectivity and dimension of the spins itself, but the common basis has remained the Hamiltonian in equation (1.1). In any case, the basic ingredient of a spin glass is the existence of frustration due to the special choice of quenched disorder in these systems. Disorder is characterized by the set of bonds of a chosen sample. Frustration occurs, if it is not possible to satisfy every single bond of a chosen model at the same time (i.e. to find a configuration of spins for which each pair of nearest neighbours $s_{i}$ and $s_{j}$ contributes as $-\frac{1}{2} J_{i j}$ in equation (1.1)). We find such a situation in Figure 1.1, which shows a small loop of four one-dimensional spins (Ising spins, sketched as arrows) with positive or negative bonds between them. In the sketched situation not all four bonds can be satisfied at the same time, as the spin in the lower left corner should point up according to the interaction with its neighbour on top resp. point down according to the interaction with its neighbour to the right.

It is not surprising that disorder and frustration complicate the investigation of spin glasses in contrast to pure ferro- or antiferromagnets. However, both in experimental and theoretical spin glasses a phase transition from a 


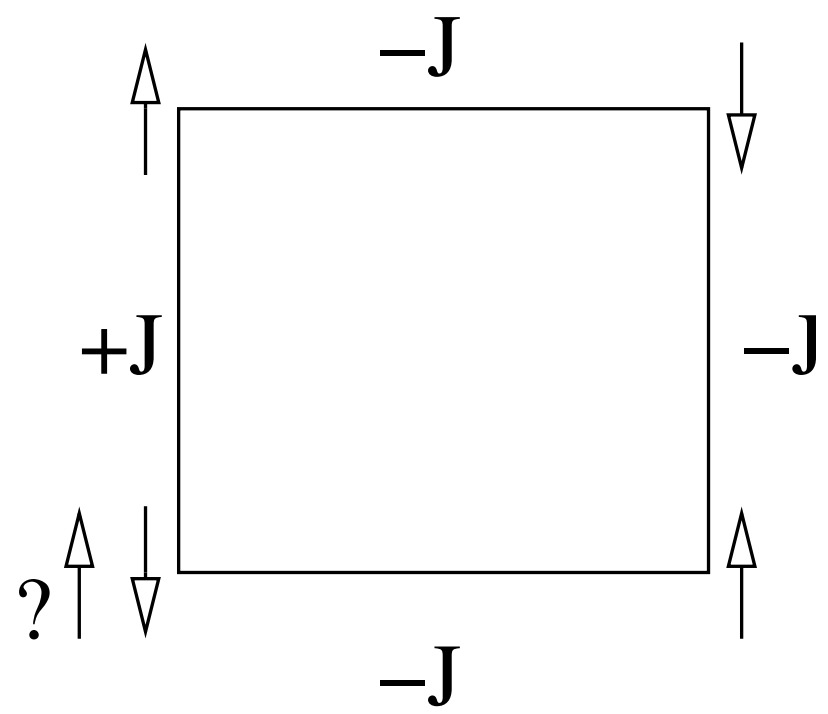

Figure 1.1: Example of a frustrated loop of four Ising spins.

paramagnetic phase to a spin glass phase is observed (though not in all models as we will see). In the paramagnetic phase, thermal fluctuations of the spins dominate and prevent to establish a preferred direction a single spin points into. In contrast, in the spin glass phase the spins become frozen in time, which means that the force of ordering to lower the energy dominates the thermal fluctuations. Due to the disorder of the bonds $J_{i j}$, the low temperature configuration of spins cannot be uniform or periodical, but is frozen in disorder. However, for finite temperatures, the spins still fluctuate, but each single one does fluctuate around its preferred direction. The analogy of the direction of the spins to the location of atoms in the glass transition (where the atoms become frozen in disorder) is responsible for the term spin glass.

Within the framework of statistical physics, the properties of a spin glass consisting of $N$ spins are described by the extensive free energy of this system, $F_{J}$, where the index $J$ denotes the special choice of disorder of this system. The free energy $F_{J}$ is equal to minus the logarithm of the partition function $Z_{J}$ times the inverse temperature $\beta=\frac{1}{T}$ as $F_{J}=-\frac{1}{\beta} \ln Z_{J}$. The partition function is found by calculating the Trace (the integral over all possible configurations of the spins) of the Boltzmann probability of all possible spin configurations, which gives a single spin configuration a statistical weight proportional to $e^{-\beta H}$ according to the Hamiltonian in equation (1.1). However, to obtain the full statistical information of a spin glass model (in order to describe, for instance, the phase transition), we have to average 
over the disorder (denoted by $E_{J}$ ) to find the mean value of the free energy $F=E_{J} F_{J}$. For large system sizes the free energy per degree of freedom of a special configuration of bonds, $f_{J}=F_{J} / N$, approaches the mean value, or the extensive part, $f_{\text {ext }}=F / N$, so that the distribution of $f_{J}$ gets peaked around $f_{\text {ext }}$ (i.e. the fluctuations around the mean value vanish with the system size. We will consider their order of magnitude in the last chapter of this thesis). In the thermodynamic limit $f_{J}$ is equal to $f_{\text {ext }}$. This is the property of self-averaging of the free energy. It is then possible to find nonanalyticities of the free energy $f_{\text {ext }}$, which separate the phase space of the spins into different phases.

The spin glass phase transition can be characterized by an order parameter. Among others we use the Edwards-Anderson order parameter $q_{E A}=\sum_{i=1}^{N}\left\langle s_{i}\right\rangle^{2}$. The thermal mean of a spin $s_{i},\left\langle s_{i}\right\rangle$, is zero in the high temperature phase due to the dominating thermal fluctuations, whereas it takes a nonzero value in the low temperature phase where the spins become frozen in time. However, in the low temperature phase the thermal mean points into various directions for the different spins and the sum $\sum_{i}\left\langle s_{i}\right\rangle$ is zero. The same is true when we regard a single spin and average its thermal mean over the disorder. This reflects the self-averaging property. Therefore we have to sum over the squared thermal mean of all spins, which is a sum of positive contributions in the spin glass phase.

In the following section we introduce the most prominent spin glass model, the Sherrington-Kirkpatrick Ising spin glass. As it is the first and most investigated spin glass model, it provides a variety of methods, notations and results which can be considered as the basis of spin glass physics. Therefore, we devote an own section to this model within this introduction and even present some of the basic technical subtleties which we expect to be helpful in order to get to know spin glasses.

\subsection{The Sherrington-Kirkpatrick Ising spin glass}

The first spin glass model which could be solved, was the infinite range model with Ising spins (one dimensional spins of length one, which can only point "up" or "down") of Sherrington and Kirkpatrick, known as the SK model [2] (infinite range means that each spin is connected to every other spin. It is assumed that infinitely many neighbours correspond to an infinite dimensional lattice). The authors were making use of the replica method, which is nowadays a standard method in statistical physics to calculate the mean free energy when it is not possible to calculate $F_{J}$ directly and average it over the disorder. There, one introduces $n$ uncoupled copies of the same 
system (the replicas) and calculates the disorder average of the product of all $n$ partition functions $Z_{J, \alpha}$, as $E_{J} \prod_{\alpha=1}^{n} Z_{J, \alpha}=E_{J}\left(Z_{J}^{n}\right)$. For the infinite range Ising spin glass $E_{J}\left(Z_{J}^{n}\right)$ is

$$
E_{J}\left(Z_{J}^{n}\right)=E_{J} \operatorname{Tr}_{s_{\alpha}} \exp \left(\sum_{\alpha=1}^{n}\left(\beta \sum_{i<j} J_{i j} s_{i}^{\alpha} s_{j}^{\alpha}+\beta h \sum_{i} s_{i}^{\alpha}\right)\right),
$$

where $\operatorname{Tr}_{s \alpha}$ denotes the Trace over all spin configurations and replicas, $h$ is an external field and $s_{i}^{\alpha}$ denotes the spin $i(i=1, \cdots, N)$ of replica $\alpha$ $(\alpha=1, \cdots, n)$. So far $n$ is integer, but we have to extend $E_{J}\left(Z_{J}^{n}\right)$ to an analytic function of $n \in \mathbb{C}$ and to take the limit $n \rightarrow 0$, to find the disorder averaged extensive free energy $F=\lim _{N \rightarrow \infty} E_{J} \log Z_{J}$ from the identity

$$
E_{J}\left(\log Z_{J}\right)=\lim _{n \rightarrow 0} \frac{1}{n}\left(E_{J}\left(Z_{J}^{n}\right)-1\right)
$$

where $Z_{J}$ is the partition function of the single spin glass. It is further necessary to take the thermodynamic limit $N \rightarrow \infty$ to obtain $F=N f_{\text {ext }}$. In the replica calculation this is done implicitly when evaluating the Trace. In principle, the replica limit $n \rightarrow 0$ should be taken first, but in practice the thermodynamic limit is taken first. This exchange of limits is still suspected to be a source of trouble in spin glass physics. In the following we will have a closer look at this pioneering computation.

\subsubsection{Replica calculation of the SK Ising spin glass and Replica Symmetry Breaking}

For the SK model of the Ising spin glass, Sherrington and Kirkpatrick calculated the $n$-times replicated partition function in equation 1.2 by averaging over the disorder, which meant to integrate over the same Gaussian distribution with mean zero and variance $\frac{J}{N}$ for each single bond $J_{i j}$, with $P\left(J_{i j}\right)=\frac{\sqrt{N}}{\sqrt{2 \pi} J} \exp \left(-\left[\frac{N J_{i j}^{2}}{2 J^{2}}\right]\right.$ ) (Scaling the variance with $\frac{1}{N}$ is necessary to obtain extensive quantities proportional to $N$ in the end), and gives

$$
E_{J}\left(Z_{J}^{n}\right) \propto \operatorname{Tr}_{s_{\alpha}} \exp \left(\frac{\beta^{2} J^{2}}{2 N} \sum_{\alpha<\beta}\left(\sum_{i} s_{i}^{\alpha} s_{i}^{\beta}\right)^{2}+\beta h \sum_{\alpha} \sum_{i} s_{i}^{\alpha}\right) .
$$

It is not possible to carry out the Trace over the spins if the partition function is of this form, due to the dependence on the squared spin variables $\left(\sum_{i} s_{i}^{\alpha} s_{i}^{\beta}\right)^{2}$. Therefore it is a useful procedure to reduce the dependence of 
the squared spin variables to a linear dependence by introducing the Gaussian integrals over the parameter $q_{\alpha \beta}$

$$
\begin{aligned}
& \exp \left(\frac{\beta^{2} J^{2}}{2 N} \sum_{\alpha<\beta}\left(\sum_{i} s_{i}^{\alpha} s_{i}^{\beta}\right)^{2}\right)= \\
& \int \prod_{\alpha<\beta} d q_{\alpha \beta} \exp \left(-\frac{N \beta^{2} J^{2}}{2} \sum_{\alpha<\beta} q_{\alpha \beta}^{2}\right) \operatorname{Tr} \exp \left(\beta^{2} J^{2} \sum_{\alpha<\beta} q_{\alpha \beta} \sum_{i} s_{i}^{\alpha} s_{i}^{\beta}\right),
\end{aligned}
$$

where we have set the external field to zero for simplicity. This reduction by Gaussian integrals is called a Hubbard-Stratonovich transformation. The integrals over the auxiliary parameters $q_{\alpha \beta}$ have to be carried out by saddle point methods, where this kind of integrals with an exponent proportional to a large parameter (in this case $N$ goes to $\infty$ in the thermodynamic limit) are approximated by taking the integrand at the value of the integration variable (here $q_{\alpha \beta}$ ), which maximizes the exponent. To include the term $\operatorname{Tr} \exp \left(\beta^{2} J^{2} \sum_{\alpha<\beta} q_{\alpha \beta} \sum_{i} s_{i}^{\alpha} s_{i}^{\beta}\right)$ from equation (1.5) into the saddle point calculation, one has to extract a factor of $N$. As taking the Trace in this expression can be done separately for each single site $i=1, \cdots, N$, one has $N$ integrals of the form $\operatorname{Tr} \exp \left(\beta^{2} J^{2} \sum_{\alpha<\beta} q_{\alpha \beta} s^{\alpha} s^{\beta}\right)$. Finally the $n$-times replicated partition function is

$$
\begin{aligned}
E_{J}\left(Z_{J}^{n}\right) \propto \int \prod_{\alpha<\beta} d q_{\alpha \beta} \exp \left(-\frac{N \beta^{2} J^{2}}{2} \sum_{\alpha<\beta} q_{\alpha \beta}^{2}\right. & \\
& \left.+N \log \operatorname{Tr} \exp \left(\beta^{2} J^{2} \sum_{\alpha<\beta} q_{\alpha \beta} s^{\alpha} s^{\beta}\right)\right) .
\end{aligned}
$$

The saddle point equations for the auxiliary parameter $q_{\alpha \beta}$ reveal a physical meaning of major importance. It is

$q_{\alpha \beta}=\frac{1}{\beta^{2} J^{2}} \frac{d}{d q_{\alpha \beta}} \log \operatorname{Tr} e^{\beta^{2} J^{2} \sum_{\alpha<\beta} q_{\alpha \beta} s^{\alpha} s^{\beta}}=\frac{\operatorname{Tr} s^{\alpha} s^{\beta} e^{\beta^{2} J^{2} \sum_{\alpha<\beta} q_{\alpha \beta} s^{\alpha} s^{\beta}}}{\operatorname{Tr} e^{\beta^{2} J^{2} \sum_{\alpha<\beta} q_{\alpha \beta} s^{\alpha} s^{\beta}}}=\left\langle s^{\alpha} s^{\beta}\right\rangle_{\text {ext }}$,

i.e. the parameters $q_{\alpha \beta}$ are the mean overlaps of the same spin $s_{i}^{\alpha}$ and $s_{i}^{\beta}$ from different replicas, where the index ext denotes the external exponent from the second line of equation (1.6). It can be shown that this definition of $q_{E A}$ is equal to the expectation value $\left\langle s_{i}^{\alpha} s_{i}^{\beta}\right\rangle$ with respect to the original partition function $E_{J} Z_{J}$. Even more, if one assumes that these parameters are independent of the special choice of $\alpha$ and $\beta$, it holds that 
$q_{\alpha \beta}=E_{J}\left\langle s_{i}^{\alpha} s_{i}^{\beta}\right\rangle=E_{J}\left\langle s_{i}^{\alpha}\right\rangle\left\langle s_{i}^{\beta}\right\rangle=E_{J}\left\langle s_{i}\right\rangle^{2}$ (where we have used that the $n$-times replicated partition function from equation $(1.2)$ factorises with respect to the different replicas). The last term is precisely the EdwardsAnderson overlap in the thermodynamic limit $q_{E A}=\sum_{i=1}^{N}\left\langle s_{i}\right\rangle^{2}$ introduced above, which indicates the phase transition. The assumption of indistinguishable replicas is called replica symmetry. It implies that there is a single equilibrium state for each temperature that each replica finds in the thermodynamic limit, i.e. a single state with a non-vanishing statistical weight in the thermodynamic limit. At first glance, it seems reasonable to assume this property, but it turned out to be wrong afterwards, as we will see below.

To find the free energy, one has to take the replica limit from equation (1.3), which means to continue this function analytically for $n \rightarrow 0$. The expansion of the exponential in equation (1.6) yields $1+N n\left(\frac{-\beta^{2} J^{2}}{2 n} \sum_{\alpha<\beta} q_{\alpha \beta}^{2}+\right.$ $\left.\frac{1}{n} \log \operatorname{Tr} e^{\beta^{2} J^{2} \sum_{\alpha<\beta} q_{\alpha \beta} s^{\alpha} s^{\beta}}\right)$, which leads to

$-\beta f_{\mathrm{ext}}=-\beta E_{J} f_{J}=\lim _{n \rightarrow 0}\left(-\frac{\beta^{2} J^{2}}{4 n} \sum_{\alpha \neq \beta} q_{\alpha \beta}^{2}+\frac{1}{n} \log \operatorname{Tr} e^{\beta^{2} J^{2} \sum_{\alpha<\beta} q_{\alpha \beta} s^{\alpha} s^{\beta}}+\frac{\beta^{2} J^{2}}{4}\right)$.

Under the assumption of replica symmetry $\left(q_{E A}\right.$ is abbreviated by $\left.q\right)$ Sherrington and Kirkpatrick found (before taking the replica limit)

$$
-\beta f_{\text {ext }}=\lim _{n \rightarrow 0}\left(-\frac{\beta^{2} J^{2}}{4 n} n(n-1) q^{2}+\frac{1}{n} \log \operatorname{Tr} e^{\beta^{2} J^{2} q \sum_{\alpha<\beta} s^{\alpha} s^{\beta}}+\frac{\beta^{2} J^{2}}{4}\right) .
$$

We note that the coefficient of $q^{2}$ is negative in the replica limit. Therefore extremising the free energy with respect to $q$ maximizes the free energy instead of the natural expectation to minimise it. This is one of the peculiar properties of the replica method. In equation 1.9 one can carry out the Trace (which introduces a Gaussian integral with parameter $z$ through a Hubbard-Stratonovich transformation) and take the replica limit $n \rightarrow 0$. The free energy then is given by

$$
-\beta f_{\mathrm{ext}}=\frac{\beta^{2} J^{2}}{4}(1-q)^{2}+\int \frac{1}{\sqrt{2 \pi}} d z e^{-\frac{z^{2}}{2}} \log \left(2 \cosh ^{2}(\beta J \sqrt{q} z)\right) .
$$

It is further possible to calculate the overlap $q$ explicitly, by extremising the free energy from equation (1.9) with respect to $q$. This gives

$$
q=\int \frac{1}{\sqrt{2 \pi}} d z e^{-\frac{z^{2}}{2}} \tanh ^{2}(\beta J \sqrt{q} z) .
$$


From the above discussion we know that the solution in the high temperature phase is $q=0$, while below the critical temperature it is non-zero. We expand the equation for small $q$, which yields $q=\int \frac{1}{\sqrt{2 \pi}} d z e^{-\frac{z^{2}}{2}}\left(\beta^{2} J^{2} q+\cdots\right)=$ $\beta^{2} J^{2} q-\mathcal{O}\left(q^{2}\right)$ (the dots and the symbol $\mathcal{O}$ indicate higher order terms in $q$ ). As long as $\beta J$ is smaller than one, the paramagnetic solution $q=0$ is the only solution of this equation. However, for $\beta J \leq 1$ a second non-zero solution for $q$ emerges and maximizes the free energy. The critical temperature of the phase transition of the SK Ising spin glass therefore is $T_{c}=J$. This result holds as long as the external field $h$ is equal to zero. If $h \neq 0$, there is no additional solution to the paramagnetic one, $q=0$, and the phase transition is removed.

However, the replica symmetric solution found by Sherrington and Kirkpatrick turned out to be wrong for the reason that it produces a negative entropy at low temperatures equal to $-\frac{1}{2 \pi}$. Even more, de Almeida and Thouless explicitly proved the failing of the replica symmetric solution by their calculation presented in Ref. [6], which showed that the replica symmetric solution is unstable against the breaking of replica symmetry. This calculation involves to expand the free energy from equation (1.8) around the replica symmetric solution (with the free energy $f_{R S}$ ) up to second order in the perturbation terms $y_{\alpha \beta}$, by substituting $q_{\alpha \beta}=q+y_{\alpha \beta}$. This leads to the partition function

$$
E_{J}\left(Z_{J}^{n}\right) \propto \int \prod_{\alpha<\beta} d q_{\alpha \beta} \exp \left[-N \beta\left(f_{R S}+\sum_{\alpha<\beta} \sum_{\gamma<\delta} y_{\alpha \beta} G_{\alpha \beta \gamma \delta} y_{\gamma \delta}\right)\right],
$$

where the quadratic form can be abbreviated by $y^{T} G y$ ( $y$ is the vector containing the different $y_{\alpha \beta}$ and $G$ is the matrix with entries $\left.G_{\alpha \beta \gamma \delta}\right)$. The matrix $G$ therefore describes the second derivative of the free energy at the replica symmetric SK solution, or in other words it is the matrix of coefficients governing the fluctuations about the SK solution. If the replica symmetric solution were stable against infinitesimal deviations from it, the matrix of coefficients of the quadratic form, $G$ (the Hessian), must not have negative eigenvalues. However, one of the eigenvalues becomes negative in the replica symmetric spin glass phase. Therefore it is necessary to break replica symmetry in a consistent way.

We point out that a related calculation in case of Ising spins on a regular lattice with short range interactions of Bray and Moore [7] yields this eigenvalue to be zero, which corresponds to a locally flat free energy along the eigenmodes of that eigenvalue (the second derivatives of the free energy at the saddle point must be positive to have a local minimum there). These directions were called massless modes. A flat free energy means that the 
question of stability cannot be answered up to second order in the deviations $y_{\alpha \beta}$.

The true solution of the SK model was found by Parisi in a series of publications [8, 9, 10] and summarized in Ref. [4] (but was confirmed to be the true solution much later in Ref. [11]). As replica symmetry is based on the assumption of a single equilibrium state each replica can find for a given temperature (leading to a single possible overlap $q_{E A}$ between the same spin taken from different replicas), it was the natural suggestion to have many different equilibrium states and multiple different overlaps $q_{\alpha \beta}$ when breaking replica symmetry. The complicated breaking scheme is based on a sequence of approximated solutions and leads to a probability distribution of the overlaps $q_{\alpha \beta}$ with support on the interval $0-q_{E A}$, in contrast to the corresponding distribution in the replica symmetric case, which consists of a single $\delta$-peak at $q_{E A}$. We try to understand, how replica symmetry breaking (RSB) works, by considering the first stage of it, one step broken replica symmetry (1-RSB). It involves to divide the $n$ replicas into $n / r_{1}$ blocks each of $r_{1}$ replicas, where $n / r_{1}$ must be integer (with $1 \leq r_{1} \leq n$ ). The overlap between replicas from the same block (which are $n\left(r_{1}-1\right)$ overlaps) is set to $q_{1}$ and to $q_{0}$ if the replicas are taken from different blocks $\left(n\left(n / r_{1}-1\right) r_{1}\right.$ overlaps). The free energy from equation (1.8) then is (compare to the replica symmetric free energy from equation (1.9) )

$$
\begin{aligned}
-\beta f_{\mathrm{ext}}=\lim _{n \rightarrow 0}\left(-\frac{\beta^{2} J^{2}}{4 n}(n\right. & \left.\left(n-r_{1}\right) q_{0}^{2}+n\left(r_{1}-1\right) q_{1}^{2}\right) \\
+ & \left.\frac{1}{n} \log \operatorname{Tr} e^{\left(\beta^{2} J^{2} \sum_{\alpha<\beta} q_{\alpha \beta} s^{\alpha} s^{\beta}\right)}+\frac{\beta^{2} J^{2}}{4}\right),
\end{aligned}
$$

where $q_{\alpha \beta}$ in the exponential has to be divided into terms with overlap $q_{0}$ and overlap $q_{1}$. It is possible to calculate equations of state determining $q_{0}$ and $q_{1}$, similar to equation (1.11). In the replica limit $r_{1}$ is in between $n \rightarrow 0$ and 1 and the free energy has to be maximized with respect to it. One finds that the transition temperature is not changed. Analysing the stability of this solution, the negative eigenvalue found by de Almeida and Thouless increases from the replica symmetric value of -0.16 to -0.01 in case of 1 RSB. In case of full RSB (which we describe in the following) it is expected to vanish and in that way produce massless modes, according to the discovery of Bray and Moore described above. Furthermore, the ground state energy of the 1-RSB solution is -0.7652 and agrees much better with numerical data than the replica symmetric one, which is approximately -0.798 [4].

The second step of replica symmetry breaking divides each of the $n / r_{1}$ blocks further into $r_{1} / r_{2}$ blocks with $r_{2}$ replicas apiece. Replicas within the same of these "new" blocks have overlap $q_{2}$, while replicas from two different 
blocks (but within the same of the $n / r_{1}$ blocks from 1-RSB) still have overlap $q_{1}$. We see that each stage of replica symmetry breaking introduces a new overlap $q_{i}$ and a new parameter $r_{i}$. They obey $n \leq r_{1} \leq r_{2} \leq \cdots \leq 1$, which reverses in the replica limit to $0 \leq r_{1} \leq r_{2} \leq \cdots \leq 1$. The free energy has to be maximized with respect to each of the overlaps $q_{i}$ and $m_{i}$. As already indicated, the order parameter $q_{\alpha \beta}$ can be characterized by the probability distribution of observing a certain overlap $q$. In case of replica symmetry there is only a single possible overlap and the probability distribution consists of a single $\delta$-peak at $q_{E A}$ (omitting the symmetry of a total reflection of all spins). Breaking replica symmetry subsequently introduces an overlap $q_{i}$ for each breaking step. After $k$ breaking steps the overlap distribution consists of $k+1 \delta$-peaks, each with a weight corresponding to the fraction of combinations of replicas with overlap $q_{i}$. With the total number of possible overlaps being $n(n-1)$, the number of combinations with overlap $q_{1}$ being $n\left(r_{1}-1\right)$ and the number of combinations with overlap $q_{0}$ being $n\left(n-r_{1}\right)$ (see above), the probability distribution of finding a given overlap $q, P(q)$, in case of $1-\mathrm{RSB}$ in the limit $n \rightarrow 0$ is

$$
P(q)=r_{1} \delta\left(q-q_{0}\right)+\left(1-r_{1}\right) \delta\left(q-q_{1}\right) .
$$

After $k$ steps of replica symmetry breaking the overlap distribution is $P(q)=$ $\sum_{i=0}\left(r_{i}-r_{i+1}\right) \delta\left(q-q_{i}\right)$, where $r_{0}=n \rightarrow 0$ and $r_{k+1}=1$. However, in case of full RSB, the number of replica symmetry breaking steps goes to infinity. In that case, the order parameter becomes a continuous function $q(x)$ and the probability distribution $P(q(x))$ has non-vanishing support on the interval 0 to $q_{E A}$, see Figure 1.2 .
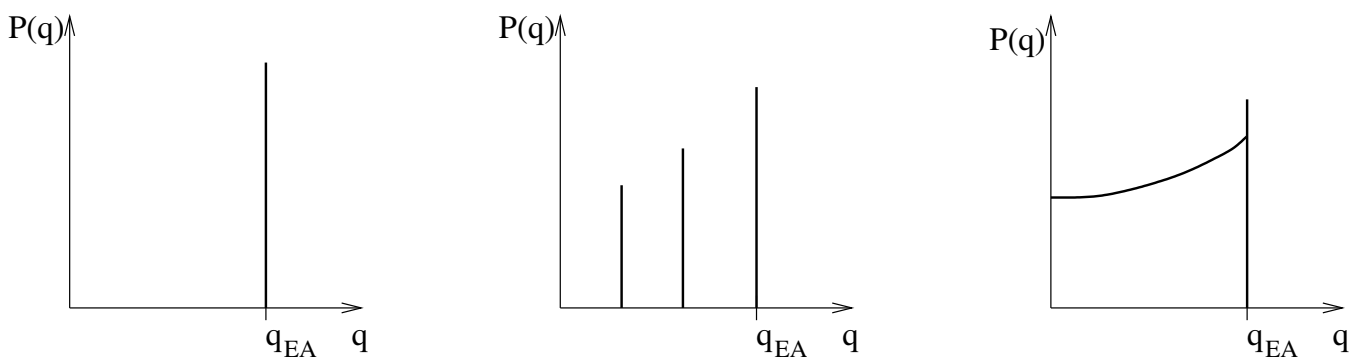

Figure 1.2: Distribution of the overlap in three different cases. Left: Replica symmetry, a single overlap at $q_{E A}$; Middle: 2-RSB, three different overlaps are possible; Right: Full RSB, the distribution has non-vanishing support between 0 and $q_{E A}$

The physical significance of breaking replica symmetry of the overlap $q_{\alpha \beta}$ into infinitely many overlaps between 0 and $q_{E A}$ lies in a multi valley structure of the phase space, as the RSB solution implies to have many different 
equilibrium states. An equilibrium state in the thermodynamic limit is defined such that its energy can not be lowered by flipping any finite fraction of spins. In finite systems these states become metastable. In a dynamical interpretation we find, that within a single valley the overlap between a certain spin at different times is equal to $q_{E A}$. If it is possible to overcome the free energy barriers separating the different valleys we find different overlap values between the current and initial state. In the thermodynamic limit the free energy barriers become infinitely high, so that the system is trapped in a single valley. Accordingly, for a large but finite system, it is possible to overcome the barriers, but it takes very long times. This property makes it hard to measure physical quantities experimentally on reasonable time scales.

\subsection{Return to Introduction}

We have already mentioned that the RSB ansatz of Parisi was proved to solve the infinite range SK model correctly in Ref. [11]. However, there has been continuing doubt that this picture holds in finite dimensions. Even more, in Ref. [12] it was suggested that broken replica symmetry can not hold in any dimension, though this proof does not give insight into the failing of the broken replica symmetry calculation. The most famous competing picture to explain the low temperature behaviour of spin glasses is the Droplet picture developed by Fisher and Huse and Bray and Moore in Ref. [13, 14, 15]. It is a phenomenological theory, which is based on the assumption of a single unique groundstate (up to the total spin reflection), i.e. it is a replica symmetric theory. Within the Droplet theory it is assumed that low-lying excitations of the groundstate follow from coherent spin flips of finite spin clusters (the droplets). These excitations dominate the equilibrium and dynamical properties of spin glasses at low temperatures. As this theory will not be subject of this thesis we do not go into detail here. However, both theories have been applied to explain fascinating non equilibrium properties of spin glasses, like aging, rejuvenation and memory effects, see Ref. [16, 17, 18, 19]. They all describe aspects of the dependence of the non-equilibrium behaviour of spin glasses on the history of the sample (as for instance waiting times, temperature dependence and external field dependence) and are strongly influenced by the slow relaxation due to the complicated free energy landscape spin glasses exhibit. For instance, aging denotes the slower relaxation for longer waiting times [19]. Rejuvenation means that a sample, initially almost equilibrated at a certain temperature has a suddenly increasing susceptibility when lowering the temperature. This increasing susceptibility describes a stronger response to this temperature change than before, i.e. it corresponds to a large 
change of the sample, which is then said to be younger (therefore the term rejuvenation). After bringing the sample back to the initial temperature $T_{1}$ it finds the initial state at that temperature, i.e. it seems to remember its own history (memory effect). A related phenomenon in spin glass physics is chaos [16]. It describes, that a small change in the temperature or in the interaction bonds $J_{i j}$ results in a large change of the spin glass sample. In the thermodynamic limit the initial and the final state are totally uncorrelated, which corresponds to a strongly changing energy landscape. We will be concerned with bond chaos in chapter 5 .

Due to the simplicity of the general microscopic spin glass model it is possible to map different problems from other areas of research onto spin glasses as a prototypical example of a disordered system and use various of the methods developed in spin glass physics in these contexts. Prominent examples of such influence of spin glass physics are the Cavity method [20] and the message passing algorithm [21], the parallel tempering Monte Carlo algorithm [22] and error correcting codes [23, 24] (where the decoding of a bit sequence (sequence of 0 and 1 ) which has been submitted through a noisy channel can be facilitated by using the representation of the bit sequence in terms of Ising spins $( \pm 1)$ and the additional information of the ground state configuration; These ideas can also be applied to image restoration [23], in the simplest case by representing the bits of a monochrome image by Ising spins and adding further information on images in general, as smoothness etc.). Even for risk minimization in financial markets, spin glass models have been applied [25, 26, 27].

Up to now, the present introduction of this thesis serves the purpose of presenting the fundamental ingredients and phenomena of spin glasses. It focusses on the first solvable model of a spin glass, the infinite ranged Ising spin glass, as the main observations of these investigations have deeply influenced theoretical spin glass physics throughout the years. The description of the replica symmetry breaking scenario of Parisi should be very helpful to follow this thesis. Applications and competing theories are less relevant here, so that we have discussed them only briefly. Now, we are going to motivate analysing a spin glass with continuous vector spins, the $m$-component spin glass, and describe how this model has emerged in spin glass physics. Furthermore, this thesis deals with a different, slightly more realistic picture of neighbourhood of the spins than the infinite range model, closer to a real spin glass in finite dimensions. For this purpose, we will make use of the Cavity method, which will be introduced in chapter 2 .

The subject of this thesis is the $m$-component vector spin glass, in which the spins are $m$-component vectors with a fixed length and $m$ is in general large (later, we will define what exactly large means). The reader might ask, 
why this is a worthwhile research topic, as the main subject of interest in spin glass physics seems to be the Ising spin glass, following the introduction of this thesis up to now. To answer this question we are going to sketch the progress spin glass physics has made over the years, on which the present thesis is footed. We will see how the analysis of the SK Ising spin glass has been extended to vector spin glasses and, on the other hand, to finite dimensional or at least diluted lattices, such as the Bethe lattice, which we are going to introduce in the next chapter. Finally, we will learn in which way and to which extend the $m$-component spin glass is a promising subject of research.

To describe the limitation of the replica theory of Parisi we mention that Replica field theory [28] extends the replica theory of Parisi to finite dimensions. Below six dimensions all calculational tools break down, which marks the upper critical dimension $d_{u}$ (above which the mean field solution - in that case the SK solution of Parisi - is probably correct). In a recent publication of Bray and Moore [29], the authors show that the de AlmeidaThouless line vanishes rapidly as $\propto(d-6)^{4}$ when approaching $d_{u}=6$ from above. This line marks the spin glass phase transition of a spin glass in an external magnetic field and is a characteristic of the broken replica symmetry solution (in the replica symmetric case there is no phase transition of a spin glass in an external field, i.e. no de Almeida-Thouless line). Furthermore, in Ref. [12] it was proved that broken replica symmetry can not hold in any dimension, though this proof is non-constructive and does not analyse the failing of the broken replica symmetric calculation in detail, nor gives it insight into the nature of the spin glass phase. Nevertheless, it is one of several hints, that going beyond the mean field solution (i.e. the fully connected spin glass) towards finitely connected models (below $d_{u}=6$ ) might restore replica symmetry.

Furthermore, it seems impossible to calculate a partition function of a finitely connected spin glass directly. Therefore simplifications are necessary to make analytical progress as for instance with the Cavity method, which uses the picture of a finite number of nearest neighbours. The appropriate lattice for this method, the Bethe lattice, is somewhere in between the fully connected and finite dimensional lattices. For vector spin glasses as the XY spin glass (two dimensional spins) and the Heisenberg spin glass (three dimensional spins) there has so far been mainly numerical progress. Nevertheless, there are continuing discussions on the properties of the phase transition, which we will mention in the following section. 


\subsection{State of the Art}

Up to now we have seen that the solution of the SK Ising spin glass exhibits quite complicated properties, finally leading to the Parisi replica symmetry breaking scenario with all its implications. The Ising spin glass is a reasonable starting point to analyse the spin glass problem, but it is a restriction as well. Of course, one is similarly interested in vector spin glasses, such as the XY spin glass (in which the spins have two components) and the Heisenberg spin glass (spins are three component vectors). However, it has so far not been possible to calculate the partition function of a vector spin glass for small component numbers. Numerical work still deals with the question of the existence of a spin glass phase transition and its connection to "chiralities" [30, 31, 32, 33, 34, 35, 36]. The picture of chiralities is based on the property of continuous spin glass models to use the additional components to lower the energy of the system compared to the discrete spin variables in the Ising spin glass. A plaquette of four spins can have negative or positive chirality if it is frustrated, or no chirality if all bonds can be satisfied at the same time. In a frustrated plaquette (as in Figure 1.1) of four XY spins the ground state of the bimodal distribution of interaction bonds $J_{i j}$ can be calculated to be such that between two neighbouring spins (going clockwise around that plaquette) with a ferromagnetic bond between them, there is an angle of $\pi / 4$. Between two spins with an antiferromagnetic bond between them, there is an angle of $\frac{5}{4} \pi$, if the sense of rotation during a clockwise trip around the plaquette is clockwise as well (positive chirality) [5]. If the rotation is counter-clockwise the chirality is negative. Such spin configurations producing different chiralities are illustrated in figure 1.3. We see that there is an extra (discrete) degeneracy additional to the infinite degeneracy due to the invariance under rotations, which is a global reflection of the spins with respect to an arbitrary axis in spin space. For Heisenberg spins a similar but three dimensional quantity can be defined. In the low temperature phase the chiralities are supposed to differ significantly from zero. As chirality is an Ising like variable, it is supposed to be possible to describe the low temperature phase similar to the Ising spin glass [31. There is continuing debate about the question whether there is a chiral-spin decoupling in the sense that chiral order occurs above the critical temperature of spin glass ordering or if there is a common transition temperature for chiral and spin glass ordering. Answering this question is complicated by the lack of an analytical solution for the regarding spin glass models.

The natural generalization of XY- and Heisenberg spins is to investigate spins with arbitrarily many components $m$, since it seems reasonable that vector spins exhibit common features as they consist of spins with a contin- 

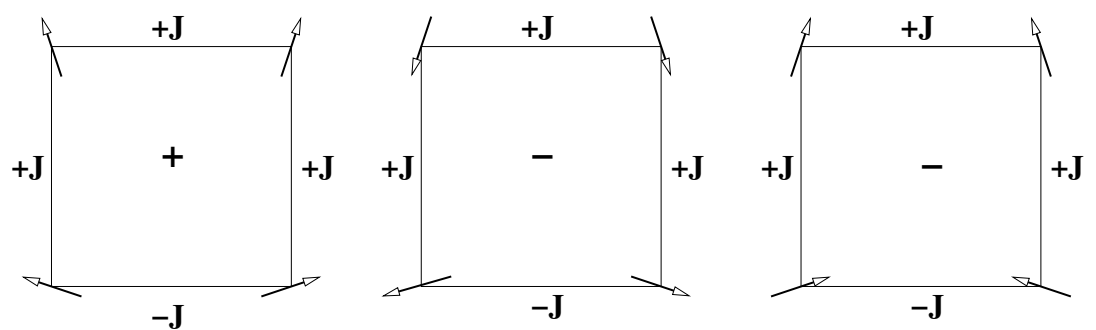

Figure 1.3: Spin configurations on frustrated plaquettes. The signs inside the plaquettes denote the sign of the local chirality. The different configurations can be generated from each other by a global reflection of the spins, but not by a global rotation.

uous symmetry and are rotational invariant in the space the spins live in. Furthermore the analysis of the $m$-component spin glass is simplified in the limit $m \rightarrow \infty$ (as we will see later), which makes it a promising starting point for investigating vector spin glasses. The motivation to study this model is even more supported by the finding that the $m$-component model of the ferromagnet is strongly related to the spherical model of the ferromagnet [37] and that the spherical model of a spin glass was solved in Ref. [38]. Therefore we will describe this model first.

Within the spherical spin glass the spins remain one dimensional but are relieved from the restriction of having a fixed length of 1 . The only constraint they have to fulfill is $\sum_{i} s_{i}^{2}=N$. As in the SK model the interactions between the spins are infinitely long ranged. The analysis is built on the Hamiltonian from equation (1.1) and on the known shape of the eigenvalue density of the connectivity matrix (whichs entries are the interaction bonds $J_{i j}$ ) in the thermodynamic limit. It corresponds to the Wigner semicircular law [39, 40]. The spherical model is solved in terms of the eigenbasis of the interaction bonds by making use of this law. This analysis results in the observation of a phase transition at $T_{c}=J$, similarly to the SK model (with $J^{2}$ being the variance of the distribution of the bonds). Furthermore it is possible to calculate the free energy and show that the ground state energy is dominated by the largest eigenvalue of the connectivity matrix. Even more, this model is replica symmetric as there is just a single order parameter and a single unique ground state.

The $m$-component spin glass is a spin glass model which consists of $N$ vector spins with $m$ components each. The spins have a fixed length $\sqrt{m}$. It was first studied within a replica calculation in the thermodynamic limit, analoguously to the one of the SK model in Ref. 41], mentioned in the introduction of this thesis. There, the authors found the spin glass phase transi- 
tion and a free energy equal to the one of the spherical spin glass in the low temperature phase by using the replica symmetric ansatz. However, they did also show that replica symmetry is broken for all finite component numbers $m$ (along the lines of the stability analysis of de Almeida and Thouless for the SK Ising spin glass sketched above). This observation was confirmed by finding massless modes in the $m$-component spin glass in the limit $m \rightarrow \infty$ as well [42, 43, 44].

Later on it was discovered that the $m$-component spin glass of finite size displays an unusual phase transition at $T=0$ in the limit $m \rightarrow \infty$, in which the spins use only a fraction of the $m$ available components and this fraction, $m_{0}$, scales with the system size as $m_{0} \sim N^{\mu_{m}}$ [45, 46, 47, 48]. In Ref. [46] this transition has been described as a generalization of a BoseEinstein condensation for which the spins condense into a $m_{0}$-dimensional subspace of the $m$-dimensional space which is actually available (in contrast to that, the condensate of the conventional Bose-Einstein condensation occupies a one-dimensional state). We keep the term generalized Bose-Einstein condensation throughout this thesis. The exponent $\mu_{m}$ depends on the underlying lattice. It is $\frac{2}{5}$ for the infinite range model and decreases for finite dimensional lattices. Apparently, this number of components, $m_{0}$, is sufficient to find the states of lowest energy, whereas for $m<m_{0}$ frustration along with a limited number of degrees of freedom leads to the complicated free energy landscape of the RSB scenario. We see that above $m_{0}$, the $m$ component spin glass becomes independent of $m$ and corresponds to the limit $m \rightarrow \infty$. In analogy to the finding of an unstable replica symmetric solution for finite $m$ in the thermodynamic limit, it is assumed to find replica symmetry in a finite system for $m>m_{0}$. For finite temperatures there is so far no reliable theory for this phenomenon, though it is expected to carry over to finite temperatures. The nature of this unusual phase transition at finite temperatures is the concern of chapter 3. The question of replica symmetry breaking when the maximally available number of components is smaller than the critical number of components for the large- $m$ limit will be treated in chapter 4. However, the generalized Bose-Einstein condensation contributes to the question of the nature of the usual spin glass phase transition, as at $T=0$ in Ref. [47] this condensation influences the nature of ordering of the spins. There, it was shown that correlations in the SK model decay with a power of the system size as $N^{-\mu_{m}}$ (whereas in a true spin glass phase transition the correlations should be finite, exhibiting long range order), which is reminiscent of the Kosterlitz-Thouless transition of the two-dimensional ferromagnet (where there is a finite temperature phase transition but no long range order below $T_{c}$ [49]). We will have a closer look at this phenomenon in chapter 2 and we will try to understand the nature 
of ordering in the low temperature phase. Furthermore the lower critical dimension of the SK model of the $m$-component spin glass has been investigated by field theoretical methods [50, 44, 43] and by calculating the finite size scaling of the zero temperature defect energy [51, 52] (the scaling of the defect energy describes the cost in energy to break up the ground state configuration). The lower critical dimension is the dimension of the lattice below which there is no finite temperature phase transition. Field theoretical methods predict a dimension of eight [44, 43], whereas numerical methods find a lower critical dimension slightly above five [51, 52]. This controversy may be at least partially resolved by the exchange of the thermodynamic limit and the large- $m$ limit. In numerical simulations the systems analyzed are of finite size, but the limit $m \rightarrow \infty$ (at $T=0: m \geq m_{0}$ ) can be taken. In analytical computations the thermodynamic limit is taken first. Though we will not be concerned with critical dimensions in this thesis (as the model we investigate is in between the infinite range and finitely connected models and not appropriate for this analysis), we will have to take care of the order of limits.

A different question for spin glasses has emerged by understanding the distribution of ground states of a spin glass as an application of extreme value statistics. In extreme value statistics there are three classical universality classes, the Weibull, Fréchet and the Gumbel distribution, and a fourth, only recently discovered distribution, the Tracy-Widom distribution (the distribution of the largest or smallest eigenvalue of a matrix with Gaussian random entries). Universality [53] has become a much-noticed property since renormalization group methods have shown that universal characteristics exist for many different models in many different phenomena, as for instance in critical scaling. The sample-to-sample fluctuations of the ground state energy (these are the fluctuations from sample to sample due to different realizations of disorder) are obviously an extreme value observable and have been investigated analytically [54, 55, 56, 57] and even more numerically [58, 59, 60, 58. The question of interest is the scaling of these fluctuations (and the sample-to-sample fluctuations of the free energy) with the system size, characterised by a scaling exponent $\mu$ and its universality. We will focus on this problem in chapter 5 and calculate the sample-to-sample fluctuations of the free energy of the $m$-component spin glass. In the literature, there have been investigations and results on three important spin glass models: The SK Ising spin glass, the Random Energy Model (REM) and the spherical spin glass. According to these, the Ising spin glass exhibits fluctuations which probably scale as $N^{1 / 6}$ (there is a competing exponent $\frac{1}{4}$ but currently also growing consensus on the value of $\frac{1}{6}[54,55]$ ). The REM consists of randomly independently distributed energies and is not described 
by a microscopic Hamiltonian, see Ref. [61, which makes it a very simple spin glass model. It has a phase transition at $T_{c}=\frac{J}{2(\ln 2)^{1 / 2}}$ with an energy of $-E_{0}$ and freezing in time below $T_{c}$. The REM is solved by a 1-RSB ansatz and its sample-to-sample fluctuations are distributed according to the Gumbel universality class and hence of order $\mathcal{O}(1)$ 62, 63].

As described above, the ground state energy of the spherical spin glass is determined by the largest eigenvalue of the interaction matrix, which contains the bonds between neighbouring spins. As the bonds are Gaussian distributed random variables, the ground state energies of the spherical spin glass are distributed according to the Tracy-Widom distribution, with $\Delta E \sim N^{1 / 3}$. Additionally it was proved that for finitely connected spin glasses the sample-to-sample fluctuations scale as $\Delta F \sim N^{1 / 2}$. We see, that different spin glass models belong to different universality classes of extreme value statistics and it is an interesting and open question whether there exist certain sub-classes of spin glasses (as for instance replica symmetric models with infinite ranged interaction) which belong to the same universality class.

\subsection{Motivation}

The reader may now understand why we have chosen the $m$-component spin glass as the subject of research. In the field of spin glasses, the SK model of the Ising spin glass has received most attention, even the sample-to-sample fluctuations have been calculated. Vector spin glasses have so far mostly been investigated numerically, but the $m$-component spin glass can be tackled analytically as well. Finite dimensional lattices are also hard to analyze. Therefore most work focussed on these lattices has been done numerically. The only way to make analytical progress seems to be the simplification resulting from tree like graphs with finite connectivity. Besides the $m$-component spin glass being a worthwhile subject to study in its own rights, the above reasons suggest that it might also have an impact on other spin glass models.

We see that there are various reasons to study the $m$-component spin glass. First of all it is a decisive question whether the $m$-component spin glass has a spin glass phase transition with a finite critical temperature below which the spins become frozen in disorder. Second, we want to know if the phase transition is accompanied by the generalized Bose-Einstein condensation and if we find replica symmetry breaking below the large- $m$ limit. Finite component numbers might be the necessary ingredient to discover the nature of the breaking of replica symmetry for finite system sizes. Third, we have to ask about the nature of the spin glass transition with respect to the generalized Bose-Einstein condensation. 
These questions will be mainly answered for the $m$-component spin glass on a Bethe lattice, i.e. for a lattice with finite connectivity appropriate to apply the Cavity method to it [20,64]. A Bethe lattice is indeed different from finite dimensional lattices such as a cubic or square lattice, but allows to make analytical progress. This different picture of neighbourhood compared to the fully connected model has so far not been intensively investigated for vector spin glasses. Only a few publications try to handle this issue [65, 66, 48]. To this end we use numerical methods such as the simulation of the configuration of effective fields (resulting from the Cavity method for the $m$-component spin glass) and the parallel tempering Monte Carlo method, but also analytical methods to gain deeper insight into the low temperature physics of the $m$ component spin glass. It will be interesting to see whether we find ordering in the low temperature phase which is reminiscent of other ordering phenomena as the picture of chirality or as Goldstone modes, which describe excitations by rotations of the (translationally invariant) spin configuration, which cost no energy and lead, for instance, to Kosterlitz-Thouless transitions [49, 53].

Furthermore we will calculate the sample-to-sample fluctuations of the free energy of the infinite range (SK) model of the $m$-component spin glass and ask how it is related to other models, especially the spherical model.

In total, this thesis gives some insight into the nature of the low temperature phase of the $m$-component spin glass. The outline is as follows. In chapter 2 we present the self-consistent effective-field approach, which adjusts the Cavity method to the case of $m$-component vector spins. The suitable lattice for this method is a Bethe lattice, i.e. a random graph where each spin has the same (finite) number of nearest neighbours. It is the distribution of effective fields which indicates the phase transition and reveals the nature of the low temperature phase. Though this derivation has already been described in [67, 48, we repeat it to enable the reader to understand this approach in detail. Based on this approach we present the numerical solution of it, which means to calculate the configuration of effective fields for single Bethe lattices, i.e. for single realizations of disorder. This numerical application is of further use in this thesis. As expected we find a phase transition. Furthermore we investigate this phase transition using a different numerical technique, the parallel tempering Monte Carlo algorithm. This method directly simulates the vector spins (whereas the self-consistent effective-field approach calculates mean effective fields acting onto the spins). Therefore, the results of both methods complement each other, though the comparison has to be done carefully.

In chapter 3 we use both numerical methods to investigate the unusual phase transition called the generalized Bose-Einstein condensation, where there is a maximum number of components the spins, resp. fields of a $\mathrm{m}$ - 
component spin glass of finite size use. In the groundstate, this phenomenon has already been observed and we review these results before we present the results for finite temperatures, where we find an interesting temperature dependence of this maximum component number. Besides this, the investigations reveal a further phenomenon, which is detected as rotations of the configurations of effective fields below the critical temperature. This strange observation is supported by similar findings from the parallel tempering algorithm. Together with the results from chapter 2 we develop a common picture of the phase transition.

In chapter 4 we show how replica symmetry is broken in our model. As already mentioned, replica symmetry is expected to hold as long as the available number of components is larger than the effective number of components the spin, resp. fields use (which we investigate in chapter 3). We confirm this conjecture with the results of simulations based on the self-consistent effective-field approach on the one hand and of the parallel tempering Monte Carlo algorithm on the other hand. Furthermore, we try to observe the breaking of replica symmetry analytically. For this purpose we finally present a method to calculate spin overlaps, which explicitly distinguishes between a replica symmetric and a broken replica symmetry ansatz (as described in the introduction). We compute and compare these overlaps for a replica symmetric and a 1-RSB ansatz.

In the final chapter 5 we present the calculation of the sample-to-sample fluctuations of the SK model of the $m$-component spin glass. This computation involves a basic analytical part as well as numerical support for a special scaling ansatz (for which we use the parallel tempering Monte Carlo algorithm). The calculation is based on an exact connection between the sampleto-sample fluctuations and bond chaos in the $m$-component spin glass. We show how our result relates to other important spin glass models and to the research field of extreme value statistics. 


\section{Chapter 2}

\section{Phase Transition}

One of the most interesting questions to answer for every spin glass model is whether it has a spin glass phase transition at a finite temperature below which the spins become frozen in disorder. The $m$-component spin glass at least has a phase transition. On the one hand, this was shown in Ref. [41] for the SK model by making use of the replica method and on the other hand in Ref. [48] for finite connectivities on a Bethe lattice using the Cavity method [20]. The transition temperature depends on the connectivity. It is unity for the SK model and decreases for decreasing connectivities [48]. In this chapter we review the self-consistent effective field approach of the $m$-component spin glass on a Bethe lattice of Aspelmeier and the author [48], as it is essential in order to understand the underlying concept of the simulations we use to analyze the low temperature physics of this model in this thesis. In addition, we will support the derivation of a spin glass phase transition by parallel tempering Monte Carlo simulations of the $m$-component spin glass. This method will be explained in detail in section 2.4. Within these simulations, the phase transition is indicated by a predicted finite size scaling of the correlations between the $N$ spins in the low temperature phase.

The definition of a Bethe lattice will be given in the following as a random graph with fixed connectivity. The self-consistent effective field approach adjusts the Cavity method on a Bethe lattice for Ising spins to the case of $m$-component vector spins. This method is based on the propagation of mean field quantities, the cavity fields, through the Bethe lattice. It is their distribution which reveals the physics of this spin glass model, as for instance the phase transition. Afterwards, we will see how it can be extended to finite component numbers. As the derivation from Ref. [48] makes use of the saddle point method for $m \rightarrow \infty$, we have to take corrections to the saddle point along (in a power series in $\frac{1}{m}$ ) to deduce the generic behaviour for large but finite component numbers. In both cases, the self-consistent effective-field 
approach yields an implicit equation determining the effective fields of the Bethe lattice, which is suited to simulate a configuration of these fields on single realizations of these Bethe lattices numerically. Results of such simulations, both for infinite and finite component numbers, will be presented. Throughout this thesis the simulation of a configuration of fields will be a major tool to investigate the low temperature phase. Concerning the phase transition we will see that the results of the simulation agree with the analytic results of Ref. 48]. However, in the following chapters we will learn that the criterion for the phase transition is not sufficient for vector spin glasses. Therefore we will have to take a closer look at the investigation of the effective fields and we will compare these results to those of a parallel tempering Monte Carlo simulation of the same model. Both investigations contribute in different ways. Whereas the effective fields are mean field quantities which are not affected by thermal fluctuations, the parallel tempering Monte Carlo algorithm uses the spins itself which are strongly affected by thermal fluctuations. Finally, we will find common support for a phase transition, but no standard freezing in disorder below $T_{c}$.

As the $m$-component spin glass was shown to be replica symmetric in the thermodynamic limit only for $m \rightarrow \infty$, the analytical basis of this chapter is restricted to that case. However, for finite $N$ we expect the $m$-component spin glass to be replica symmetric for finite, large $m$ as well. We will give further support for this conjecture in the following chapters and use it therefore already in this chapter. Finally, we present the computation of the free energy of the $m$-component spin glass on a Bethe lattice in terms of the cavity fields in section 2.5 .

\subsection{The Cavity method and the self-consistent effective-field approach}

The Cavity method cannot be used on any arbitrary lattice. It has to have a locally treelike and homogeneous structure, in order to have the same statistical properties at every site. A Bethe lattice is a random graph with fixed connectivity. Two realisation of such a graph for 6 spins with a fixed connectivity of 3 are provided in figure 2.1. For such random graphs, loops are large (of order $\log N$ ), so the structure is locally treelike and the feedback of a single spin onto itself is small. Nevertheless, loops are present and introduce frustration. A Bethe lattice is a homogeneous system, as it has no boundaries and every single spin has the same number of nearest neighbours. If the spins $s_{i}$ and $s_{j}$ are nearest neighbours, the exchange interaction between them is 

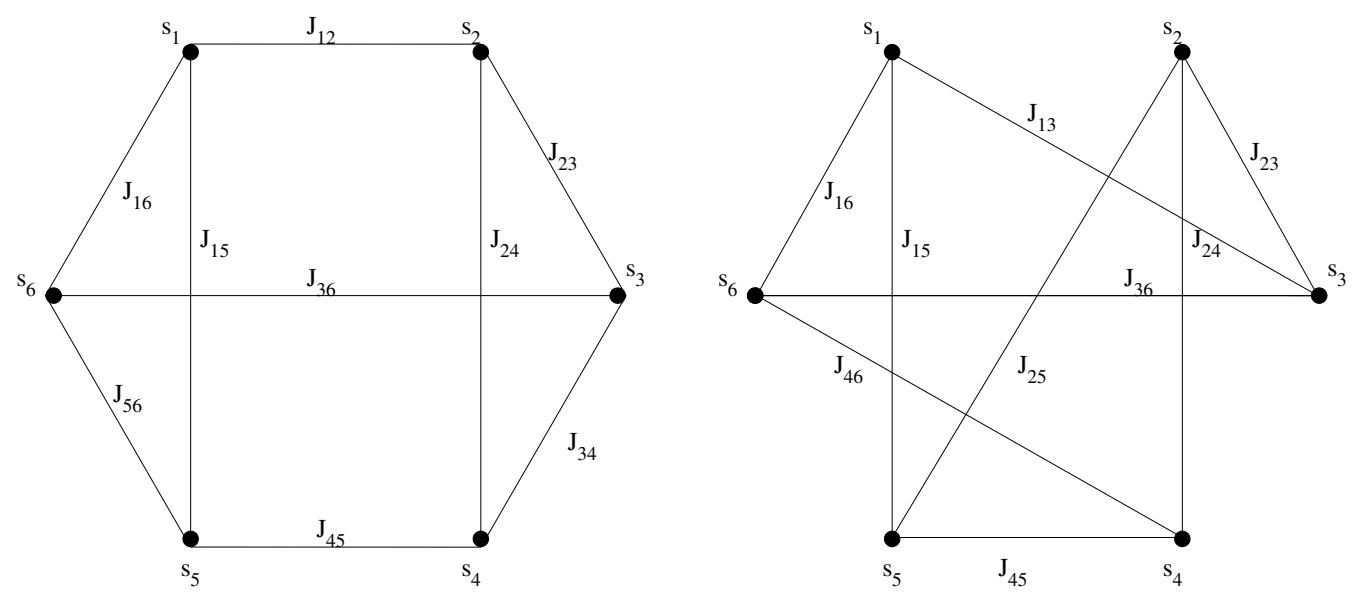

Figure 2.1: Two examples of six spins on a Bethe lattice with fixed connectivity of three.

denoted by $J_{i j}$, and it is drawn from a probability distribution $P\left(J_{i j}\right)$. Results of the following calculation will be shown for a Gaussian and a bimodal distribution, the $\pm J$ model. The width of this distribution, $J^{\prime}$, is scaled with the number of nearest neighbours like $J^{\prime}=J / \sqrt{k}$ with $J$ fixed to ensure easy comparison with the fully connected model, where $J^{\prime}=J / \sqrt{N}$ (where $k+1$ is the number of nearest neighbours on a Bethe lattice). The Hamiltonian of the model is

$$
H=-\sum_{\langle i j\rangle} J_{i j} \vec{s}_{i} \vec{s}_{j}
$$

where the sum runs over all pairs of nearest neighbours and the spins are $m$-component vectors with a fixed length, equal to $\sqrt{m}$.

The self-consistent effective field approach we use to analyse the $m$ component spin glass is an extension of the Cavity method for the Bethe lattice, developed for Ising spins by M. Mézard and G. Parisi [20]. There the key quantities are cavity fields. A cavity field $h_{i j}$ is the remaining field on a chosen spin $s_{i}$ when removing the link $J_{i j}$ to the neighbouring spin $s_{j}$ virtually from the lattice. It is furthermore understood to be the thermally averaged mean field of the remaining lattice in that situation. A cavity field is not the true field acting on a chosen spin $s_{i}$, but a technical construction. Regarding a Bethe lattice with connectivity $k+1$, all of the $k+1$ nearest neighbours contribute to produce a true local field $H_{i}$ onto spin $s_{i}$. In contrast, only each $k$ of them produce the different $(k+1$ possible) cavity fields. Propagating through a lattice of connectivity four as in figure 2.2(a), one finds the cavity field on cavity spin $s_{0}$ (virtually removing the link $J_{4}$ to the 

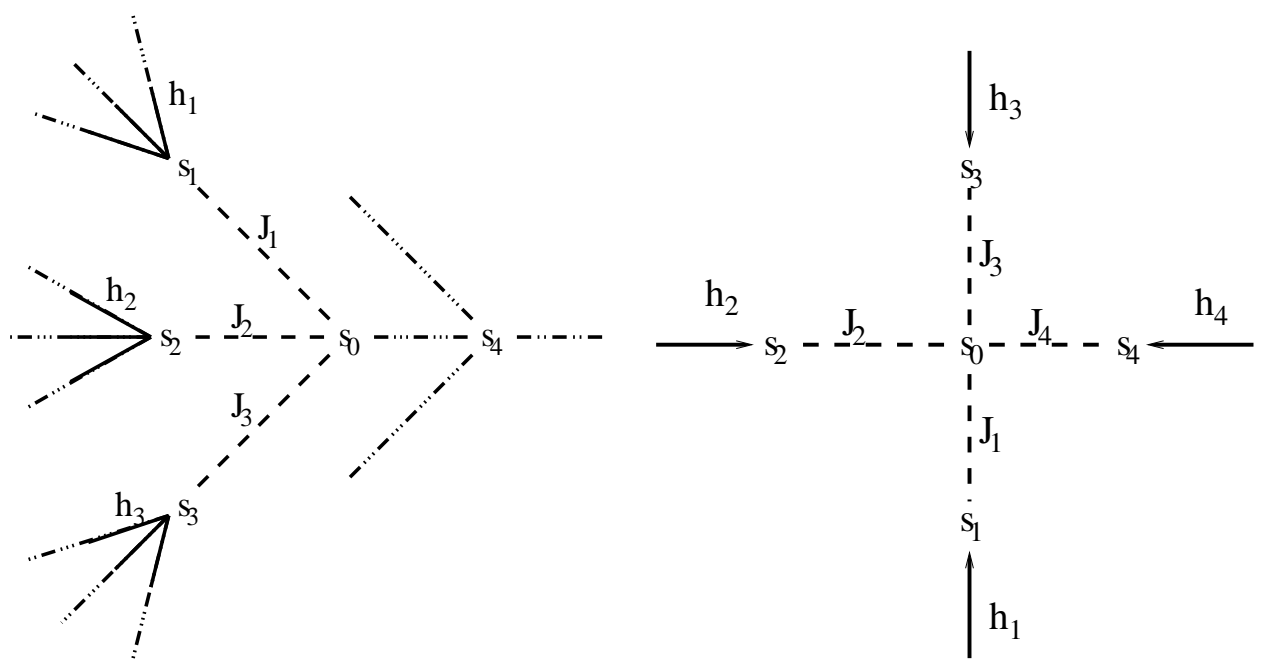

Figure 2.2: Left: The three spins $\vec{s}_{1}, \vec{s}_{2}$ and $\vec{s}_{3}$ and their back branches are merged onto spin $\vec{s}_{0}$ with coupling constants $J_{1}, J_{2}$ and $J_{3}$ in this example with $k=3$. In the next step of the iteration, $\vec{s}_{0}$ and two other branches will be merged onto the spin $\vec{s}_{4}$. Right: In contrast, the true local field $\vec{H}_{0}$ onto the spin $\vec{s}_{0}$ is the sum of the interaction with all $k+1$, here 4 , of its neighbours. See text for further explanation.

spin $s_{4}$ ) to depend on the cavity fields $h_{1}$ to $h_{3}$ of the spins $s_{1}$ to $s_{3}$. This is the essential procedure of the cavity method for finite conectivity lattices: One merges $k$ branches of the lattice onto the single cavity spin $s_{0}$, each assigned a cavity field $h_{1} \ldots h_{k}$ for its last spins $s_{1} \ldots s_{k}$, where each of those spins has only $k$ nearest neighbours before merging. The cavity field onto the spins $s_{1} \ldots s_{k}$ of each branch represents the interaction of the many back spins of that branch. Before merging, the cavity fields $h_{1} \ldots h_{k}$ are statistically independent in the thermodynamic limit (as loops are of order $\log N$ ). After merging, $s_{0}$ is the last spin of the emerging single branch and has $k$ nearest neighbours $s_{1} \ldots s_{k}$ just like these new nearest neighbours had before merging. Now we understand why the treelike and homogeneous structure of the underlying lattice is important. First of all, the fields $h_{1}, \cdots h_{k}$ are assumed to be statistically independent before merging, which is justified due to the rareness of small loops of the locally treelike lattice. Second, only for a homogeneous lattice we can characterize each of the cavity fields by the same statistical properties.

The merging introduces exchange interactions $J_{1} \ldots J_{k}$ between $s_{0}$ and its neighbours, drawn independently from some probability distribution $P\left(J_{i}\right)$. Therefore $s_{0}$ can be assigned a cavity field $h_{0}$, which is found by tracing 
out the neighbouring spins $s_{1} \ldots s_{k}$ from a partition function $Z_{0}$ with the Hamiltonian of the regarded branch with the cavity spin $s_{0}, \mathcal{H}_{0}$,

$$
\mathcal{H}_{0}=-\sum_{i=1}^{k} J_{i} s_{1} s_{0}-\sum_{i=1}^{k} h_{i} s_{i}
$$

The reader should notice that in general the spin $s_{0}$ contributes to its own cavity field via the loops of the lattice. However, in the thermodynamic limit, these loops become large and the feedback becomes small. The calculation of the Trace for $\operatorname{Tr}_{s_{1} \ldots s_{k}} e^{-\beta \mathcal{H}_{0}}$ gives $\left.\left[\prod_{i} c\left(J_{i}, h_{i}\right)\right)\right] \exp \left[\sum_{i=1}^{k} \beta u\left(J_{i}, h_{i}\right) s_{0}\right]$ with $c(J, h)=2 \frac{\cosh (\beta J) \cosh (\beta h)}{\cosh (\beta u(J, h))}$ and $\beta u(J, h)=\operatorname{atanh}[\tanh (\beta J) \tanh (\beta h)]$. We remark that the Cavity method is only successful if the partition function $Z_{0}$ is found to be of the form $\sim \operatorname{Tr}_{s_{0}} e^{\beta \sum_{i=1}^{k} v\left(J_{i}, h_{i}\right) s_{0}}$ after tracing out the spins $s_{1} \ldots s_{k}$ (with a function $v(J, h)$ that depends on the dimension of the spins and the resulting evaluation of the Trace from that dimension - in the Ising spin case $v(J, h)=u(J, h))$. As the partition function $Z_{0}$ of the spin $s_{0}$ can additionally be written in terms of a single cavity field in equation (2.2) $\mathcal{H}_{0}=$ $-h_{0} s_{0}$ after merging, we find the magnetization $m_{0}=\left\langle s_{0}\right\rangle_{c}$ to be equal to $\tanh \left(\beta h_{0}\right)$ in that case $\left(\left\langle s_{0}\right\rangle_{c}\right.$ is the thermal mean of $s_{0}$ in cavity, i.e. with $J_{k+1}$ removed). In the original description it is equal to $\tanh \left(\beta \sum_{i=1}^{k} u\left(J_{i}, h_{i}\right)\right)$, which leads to the cavity equation $h_{0}=\sum_{i=1}^{k} u\left(J_{i}, h_{i}\right)$, from which the field $h_{0}$ can be calculated recursively from the cavity fields $h_{1}, \cdots, h_{k}$ before merging. A similar consideration (with $k \rightarrow k+1$ ) allows one to derive the true local field $H_{0}=\sum_{i=1}^{k+1} u\left(J_{i}, h_{i}\right)$ by merging $k+1$ branches of the lattice instead of $k$, see figure 2.2(b). It should be emphasized that the cavity fields, as well as the local fields, are thermally averaged quantities by definition and are not subject to thermal fluctuations.

The method of choice in Ref. [20] to calculate the critical temperature of the Ising spin glass on a Bethe lattice uses the distribution of cavity fields. The existence of such a distribution is assumed, as all cavity fields for any realization of disorder should display the same statistical properties. As the relation $h_{0}=\sum_{i=1}^{k} u\left(J_{i}, h_{i}\right)$ describes how to calculate any of the $k+1$ cavity fields of any spin on the lattice, it restricts the distribution of cavity fields $Q(h)$ to those fields which satisfy this relation. Therefore, the distribution of cavity fields is the solution of the functional fixpoint equation

$$
Q(h)=E_{J} \int\left(\prod_{i=1}^{k} d h_{i} Q\left(h_{i}\right)\right) \delta\left(h-\sum_{i=1}^{k} u\left(J_{i}, h_{i}\right)\right) .
$$

The symbol $E_{J}$ denotes the disorder average. Equation (2.3) reflects the cavity procedure from above, for $h_{0} \rightarrow h$. Before merging, the fields $h_{i}$ on the 
right hand side are independently identically distributed random variables drawn from the probability distribution $Q\left(h_{i}\right)$. After merging, on the one hand the new cavity field can be calculated via $h=\sum_{i=1}^{k} u\left(J_{i}, h_{i}\right)$ and on the other hand it is a random variable from the same probability distribution $Q(h)$. On average, all sites have identical statistical properties, therefore we expect them to be elements of the same distribution. Equation (2.3) holds as long as there is a single distribution of cavity fields, which is true in case of replica symmetry. If replica symmetry is broken, as it is in the case of Ising spins below $T_{c}$, the distribution of cavity fields becomes a functional distribution of distributions of cavity fields, according to the first step of the complicated breaking scheme of replica symmetry [20]. As the concern of this thesis is the $m$-component spin glass in the replica symmetric large- $m$ limit, we will not give the details of this procedure, but remark that in the broken replica symmetric case, there are many states the system can be in. Therefore a single cavity field can take different values and it takes a distribution of fields for every cavity field to describe the situation correctly. This leads to a distribution of distributions of cavity fields for the whole Bethe lattice.

The distribution of true local fields, which regards the interaction of a spin with all $k+1$ of its neighbours, is completely determined by the distribution of cavity fields. In case of replica symmetry it is

$$
Q_{l}(H)=E_{J} \int\left(\prod_{i=1}^{k} d h_{i} Q\left(h_{i}\right)\right) \delta\left(H-\sum_{i=1}^{k+1} u\left(J_{i}, h_{i}\right)\right)
$$

i.e. once the distribution of cavity fields is found, the local fields are calculated by $H=\sum_{i=1}^{k+1} u\left(J_{i}, h_{i}\right)$.

The self-consistent effective field approach of the Cavity method for the $m$-component spin glass is based on the same procedure as above, but has to be adjusted for that case. The complication arises from the fact, that a formulation of the problem in terms of cavity vector fields, $\mathcal{H}_{0}=-\vec{h}_{0} \vec{s}_{0}$, is not sufficient. We will see below why this is the case. For this reason, we have to resort to a full formulation of the problem, which means to take along higher order terms of the interaction between the cavity spin and the cavity field of the form

$$
\mathcal{H}_{0}=-\vec{h}_{0} \vec{s}_{0}-{\overrightarrow{s_{0}}}^{T} A_{0} \overrightarrow{s_{0}}-B_{0}\left(\overrightarrow{s_{0}}\right)
$$

Additionally to the linear term $-\vec{h}_{0} \vec{s}_{0}$, we have introduced the second order term with a symmetric traceless matrix $A_{0}$ and collect terms of order $\vec{s}_{0}{ }^{3}$ and higher in the function $B_{0}\left(\vec{s}_{0}\right)$. Choosing the matrix $A_{0}$ to be traceless can be done without loss of generality, since the constraint $\vec{s}_{0}{ }^{2}=m$ would only 
give constant terms for $\operatorname{Tr}_{s_{0}}^{T} A_{0} \vec{s}_{0}$. The explicit dependence of the function $B_{0}\left(\vec{s}_{0}\right)$ on $\vec{s}_{0}$ can be omitted, since this term will be neglected in the vicinity of the phase transition, where we will be able to solve the cavity equations determining $h_{0}$ and $A_{0}$. We continue to repeat the procedure from above. Before merging the $k$ branches of the lattice onto the cavity spin $\vec{s}_{0}$, each last spin $\vec{s}_{1} \ldots \vec{s}_{k}$ of these branches is assigned a full cavity field, with the same Hamiltonian as for $\vec{s}_{0}$ in equation $2.5, \mathcal{H}_{i}=-\vec{h}_{i} \vec{s}_{i}-\vec{s}_{i}^{T} A_{i} \vec{s}_{i}-B_{i}\left(\vec{s}_{i}\right)$. We have to calculate the Trace of the partition function

$$
\begin{aligned}
Z_{0} & =\int\left(\prod_{i=0}^{k} d s_{i}^{m} \delta\left(m-\vec{s}_{i}{ }^{2}\right)\right) \exp \left[-\beta \sum_{i=1}^{k}\left(-J_{i} \vec{s}_{1} \vec{s}_{0}+\mathcal{H}_{i}\right)\right] \\
& =\int d s_{0}^{m} \delta\left(m-\vec{s}_{0}{ }^{2}\right) \prod_{i=1}^{k} Z_{i} \quad, \text { where } \\
Z_{i} & =\int d s_{i}^{m} \delta\left(m-\vec{s}_{i}{ }^{2}\right) \exp \left(-\left.\beta \mathcal{H}_{i}\right|_{\vec{h}_{i} \rightarrow J_{i} \vec{s}_{0}+\vec{h}_{i}}\right) .
\end{aligned}
$$

The task is to express equation 2.6 in the form $\sim e^{-\beta \mathcal{H}_{0}}$, with $\mathcal{H}_{0}$ from equation 2.5 and find self-consistent equations for $\vec{h}_{0}, A_{0}$, and higher orders, each depending on the fields before merging, $\vec{h}_{1} \ldots \vec{h}_{k}, A_{1} \ldots A_{k}$ etc. as it happens to be the case for Ising spins (where $h_{0}=\sum_{i=1}^{k} u\left(J_{i}, h_{i}\right)$ ). We will now proceed in two ways. First, we will analyse the problem solely in terms of the vector fields $\vec{h}$ and show that a phase transition occurs at finite temperature. To do so, we will derive the cavity equation for $\vec{h}_{0}$ by the approach, that $Z_{0}$ from equation (2.6) produces the same expectation value of $\vec{s}_{0},\left\langle\vec{s}_{0}\right\rangle_{c}$, as if this spin was only subject to the single field $\vec{h}_{0}$. We remark that this is the cavity expectation value and should not be mixed up with the true magnetisation for which $\vec{s}_{0}$ is subject to a true local field $\vec{H}_{0}$. This computation was achieved in Ref. [67, 48] and was called the self-consistent effective-field approach there. Second, we will solve the cavity equations by taking the matrix term $\left(\vec{s}_{i}^{T} A_{i} \vec{s}_{i}\right)$ along. This calculation is only possible in the vicinity of the phase transition. We will see that the phase transition temperature is not affected by this, and that the formulation in terms of the vector fields $\vec{h}_{i}$ is the first order approach to the full problem. Therefore it should yield the correct behaviour of the $m$-component spin glass close to the critical temperature.

\subsubsection{Self-consistent effective-field approach}

The self-consistent effective field approach assumes that there is a cavity field $\vec{h}_{0}$, which effectively produces the same spin expectation value $\left\langle\vec{s}_{0}\right\rangle_{c}$ as the 
cavity partition function (2.6), when the spin $\vec{s}_{0}$ is solely subject to this field. The calculation of both expectation values is done in appendix A. It is based on the saddle point approach where the large parameter is the number of components $m$. There we see, that a description solely in terms of cavity vector fields is not sufficient and why it is necessary to use the self-consistent effective field approach.

From the appendix A, the desired effective cavity field is

$$
\vec{h}_{0}=\sum_{i=1}^{k} \frac{2 \beta J_{i} \vec{h}_{i}}{4 z_{i}^{0}}
$$

with $z_{i}^{0}=\frac{1}{4}\left(1+\sqrt{1+4 \beta^{2}\left(J_{i}^{2}+\frac{2 J_{i} \vec{h}_{i}\left\langle\vec{s}_{0}\right\rangle_{c}}{m}+\frac{\vec{h}_{i}^{2}}{m}\right)}\right)$. The field depends on the interaction bonds $J_{1}, \cdots, J_{k}$ between $\vec{s}_{0}$ and $k$ of its neighbours and on the cavity fields $\vec{h}_{1}, \cdots, \vec{h}_{k}$ onto these neighbours (not taking the interaction with $\vec{s}_{0}$ into account). Furthermore, it determines the distribution of effective cavity fields in the functional fixpoint equation (compare to equation (2.3))

$$
Q(\vec{h})=E_{J} \int\left(\prod_{i=1}^{k} d^{m} h_{i} Q\left(\vec{h}_{i}\right)\right) \delta\left(\vec{h}-\vec{h}_{0}\left(\left\{J_{i}\right\},\left\{\vec{h}_{i}\right\}\right)\right),
$$

where $\vec{h}_{0}\left(\left\{J_{i}\right\},\left\{\vec{h}_{i}\right\}\right)$ is the solution of equation 2.7). As in the Ising spin glass case, the true local field $\vec{H}_{0}$ is obtained by taking all $k+1$ neighbours in the sum of equation (2.7) into account

$$
\vec{H}_{0}=\sum_{i=1}^{k+1} \frac{2 \beta J_{i} \vec{h}_{i}}{4 z_{i}^{0}}
$$

The distribution of local fields then is $Q_{l}(\vec{H})=E_{J} \int\left(\prod_{i=1}^{k+1} d^{m} h_{i} Q\left(\vec{h}_{i}\right)\right) \delta(\vec{H}-$ $\sum_{i=1}^{k+1} \frac{2 \beta J_{i} \vec{h}_{i}}{4 z_{i}^{0}}$.

Equation 2.7) is important for us for two reasons. On the one hand, the expansion for small fields of it can be used to observe the phase transition by finding the instability of the high temperature solution $Q(\vec{h})=\delta(\vec{h})$ of the functional fixpoint equation 2.8]. This has been achieved in Ref. [67] and we repeat it for the sake of completeness in the following. On the other hand, we will use it as a prescription to iterate the effective fields of a given Bethe lattice numerically and obtain their precise values. Besides the emerging of the phase transition, this numerical study will exhibit further insight into the low temperature physics of the $m$-component spin glass in the following 
chapters, as, for instance, the number of necessary components to form a stable replica symmetric configuration of effective fields and the breaking of replica symmetry.

To find the critical temperature, we have to answer the question how the distribution of effective fields $Q(\vec{h})$ is related to the spin glass phase transition. As the spin glass phase transition is indicated by the Edwards-Anderson order parameter $q_{E A} \sim E_{J}\left\langle\vec{s}_{i}\right\rangle^{2}$, which is zero above $T_{c}$ and increases below (while $E_{J}\left\langle\vec{s}_{i}\right\rangle$ remains zero even in the low temperature phase), we can link the order parameter to the variance of the distribution of effective fields, $(\Delta h)^{2}=\left[{\overrightarrow{h_{i}}}^{2}\right]-\left(\left[\overrightarrow{h_{i}}\right]\right)^{2}$ (the angular brackets denote the average with respect to the effective field distribution), with $\left\langle\vec{s}_{i}\right\rangle \sim \vec{H}_{i}$ and $\left\langle\vec{s}_{i}\right\rangle_{c} \sim \vec{h}_{i}$ from equation A.5 (and from that $E_{J}\left\langle{\overrightarrow{s_{i}}}^{2}\right\rangle \sim\left[{\overrightarrow{H_{i}}}^{2}\right]$ and $E_{J}\left\langle{\overrightarrow{s_{i}}}^{2}\right\rangle_{c} \sim\left[{\overrightarrow{h_{i}}}^{2}\right]$ ). As already pointed out, the distribution of the local fields $\vec{H}_{i}$ is fully determined, once the distribution of cavity fields is found. Therefore, $(\Delta h)^{2}$ is an appropriate order parameter with a single caveat due to the additional rotational degrees of freedom, as will be described in more detail below. Equation A.5 further says that the fields point into fixed directions in the low temperature phase for a given set of bonds $J_{i j}$, and the spins fluctuate around this preferred direction. Due to the fact, that the distribution of effective fields is averaged over the disorder, the first momentum, $\left[\vec{h}_{i}\right]$, will be zero, and we find the connection $q_{E A} \sim\left[\vec{h}^{2}\right]=\int d^{m} h \vec{h}^{2} Q(\vec{h})$. However, this criterion for the phase transition is not sufficient for vector spin glasses, due to the property of being invariant under rotations. In this case it is possible to find a stable variance $(\Delta h)^{2}$ but with a rotating configuration of fields. We will discuss this problem in the following sections.

To calculate $\left[\vec{h}^{2}\right]$, we expand equation (2.7) in first order for small fields, as should be valid close to the critical temperature and sufficient to observe the phase transition. With $z_{i}^{00}=\frac{1}{4}\left(1+\sqrt{1+4 \beta^{2} J_{i}^{2}}\right)$ (the solution of $z_{i}^{0}$ for small fields), the functional fixpoint equation (2.8) becomes

$$
Q(\vec{h})=E_{J} \int\left(\prod_{i=1}^{k} d^{m} h_{i} Q\left(\vec{h}_{i}\right)\right) \delta\left(\vec{h}-\sum_{i=1}^{k} \frac{2 \beta J_{i} \vec{h}_{i}}{4 z_{i}^{00}}\right) .
$$

The distribution of true local fields $Q_{l}(\vec{H})=E_{J} \int\left(\prod_{i=1}^{k+1} d^{m} h_{i} Q\left(\vec{h}_{i}\right)\right) \delta(\vec{h}-$ $\left.\sum_{i=1}^{k+1} \frac{2 \beta J_{i} \vec{h}_{i}}{4 z_{i}^{00}}\right)$ is closely related to the distribution of effective fields (2.10), as its variance will only be nonzero, if the variance of the distribution of effective fields is. To check whether the high temperature solution of equation (2.10), $\delta(\vec{h})$, is stable for a certain temperature, we regard $Q\left(\vec{h}_{i}\right)$ to be Gaussian prepared with a small variance $\epsilon^{2}$, i.e. we introduce a small perturbation 
Table 2.1: Critical temperature from the self-consistent effective field approach

\begin{tabular}{|c|c|c|}
\hline$k+1$ & $T_{c}$ Gaussian Bonds & $T_{c} \pm J$-model \\
\hline \hline 3 & 0.28 & $1 / 2$ \\
\hline 4 & 0.44 & $2 / 3$ \\
\hline 6 & 0.61 & $4 / 5$ \\
\hline 8 & 0.7 & $6 / 7$ \\
\hline 12 & 0.79 & $10 / 11$ \\
\hline
\end{tabular}

to the $\delta$-solution. With it, we calculate one iteration step via $(2.10)$ and compare the resulting variance of $Q(\vec{h}), \epsilon^{\prime 2}$, to the initial one, $\epsilon^{2}$. As the resulting variance depends on the temperature, we explicitly find the critical temperature, above which the variance diminishes, indicating the stability of the high temperature solution, and below which it increases, indicating the low temperature phase with a nonzero variance of the distribution. This line of argumentation is restricted to the statistical properties of the disorder averaged distribution of effective fields. Later on we will see, that it is possible to analyse the phase transition of the $m$-component spin glass on a single Bethe lattice by calculating the special non-disorder averaged configuration of effective fields on this lattice.

For small fields, the calculation of an iteration step of the distribution of effective fields involves only Gaussian integrals and we find

$$
\epsilon^{\prime 2}=4 \beta^{2} \epsilon^{2} k \int d J_{1} P\left(J_{1}\right) \frac{J_{1}^{2}}{\left(4 z_{1}^{00}\right)^{2}} .
$$

Equating $\epsilon$ and $\epsilon^{\prime}$, we obtain the critical temperature for the $\pm J$-model

$$
T_{c}=J\left(1-\frac{1}{k}\right) .
$$

In the limit $k \rightarrow \infty$, this agrees with the critical temperature of the fully connected model $T_{c}=J$ [41]. For a Gaussian distribution of bonds, the inverse critical temperature is given by the solution of the integral equation

$$
1=4 \beta^{2} \int \frac{d x}{\sqrt{2 \pi} J} \frac{x^{2} e^{-x^{2} / 2 J^{2}}}{\left(1+\sqrt{1+4 \beta^{2} x^{2} / k}\right)^{2}}
$$

which also yields $T_{c}=J$ in the limit $k \rightarrow \infty$. Equation 2.13 can be evaluated numerically. The results of the critical temperature for finite connectivities can be found in table 2.1. The reader should have in mind that 
we have used the expansion of the cavity equation for $\vec{h}_{0}=\sum_{i=1}^{k} \frac{\beta J_{i} \vec{h}_{i}}{2 z_{i}^{0}}$ for small fields, for which $z_{i}^{0} \rightarrow z_{i}^{00}$ in this equation becomes independent of the cavity fields. This approach is valid above and at the critical temperature, below which the components of the cavity fields adopt non-zero values and the original cavity equation is more appropriate to describe the physics of the system (though it is analytically much harder to handle).

Having calculated the phase transition of the $m$-component spin glass on the Bethe lattice with the self-consistent effective field approach, we have to ask whether the extension towards higher orders of the full formulation of the cavity method for this model in equation 2.5) (as $\vec{h}_{i} \vec{s}_{i} \rightarrow \vec{h}_{i} \vec{s}_{i}+\vec{s}_{i}^{T} A_{i} \vec{s}_{i}+$ $\left.B_{i}\left(\vec{s}_{i}\right)\right)$ confirms our findings. Therefore, we have to calculate the cavity partition function, equation (2.6), for the full Hamiltonian in the following section.

\subsubsection{Analysis of the full problem}

In this section we review the calculations involving higher order terms of Ref. [48] and described in equation (2.5), and see, that the transition temperature is solely determined by the formulation in terms of the vector fields $\vec{h}_{i}$ from the previous section. The general procedure of computing the critical temperature is the same here, but in detail it needs to be extended perturbatively. We use the Hamiltonian from equation (2.5) and calculate the partition functions $Z_{i}$ to

$$
\begin{aligned}
Z_{i} & =\int_{-i \infty+c}^{i \infty+c} \frac{d z}{2 \pi} \int d^{m} s_{i} \exp \left\{\beta\left(\tilde{\vec{h}}_{i} \vec{s}_{i}+\vec{s}_{i}^{T} A_{i} \vec{s}_{i}+B_{i}\left(\vec{s}_{i}\right)\right)+z\left(m-\vec{s}_{i}^{2}\right)\right\} \\
& =\int_{-i \infty+c}^{i \infty+c} \frac{d z}{2 \pi} \exp \left\{m z+\frac{\beta}{4} \tilde{\vec{h}}_{i}^{T}\left(\frac{z}{\beta}-A_{i}\right)^{-1} \tilde{\vec{h}}_{i}-\frac{1}{2} \ln \operatorname{det}\left(\frac{z}{\beta}-A_{i}\right)+\frac{m}{2} \ln \pi\right\} \\
& \times \exp \left\{\left\langle B_{i}\right\rangle_{i}+\frac{1}{2}\left(\left\langle B_{i}^{2}\right\rangle_{i}-\left\langle B_{i}\right\rangle_{i}^{2}\right)+\cdots\right\}
\end{aligned}
$$

The field $\tilde{\vec{h}}_{i}$ substitutes $\frac{\vec{h}_{i}}{m}$. The angular brackets $\langle\cdot\rangle_{i}$ denote the average with respect to the weight $\exp \left\{\beta\left(\tilde{\vec{h}}_{i} \vec{s}_{i}-\vec{s}_{i}^{T}\left(\frac{z}{\beta}-A_{i}\right) \vec{s}_{i}\right)\right\}$ and the term $\frac{z}{\beta}-A_{i}$ is a short hand notation for the matrix expression $\frac{z}{\beta} \mathbb{1}-A_{i}$. Similar to the previous section, the remaining integration over $z$ will be executed by saddle point methods. The saddle point equation for $z$ is

$$
1=\frac{1}{4}\left(\tilde{\vec{h}}_{i}^{\prime}\right)^{T}\left(\frac{z}{\beta}-A_{i}\right)^{-2} \tilde{\vec{h}}_{i}^{\prime}+\frac{1}{2 m \beta} \operatorname{Tr}\left(\frac{z}{\beta}-A_{i}\right)^{-1}-\frac{1}{m} \frac{\partial\left\langle B_{i}\right\rangle_{i}}{\partial z}+\cdots,
$$


It is possible to calculate $\left\langle B_{i}\right\rangle_{i}$ by saddle point methods. However, our concern is the approach close to $T_{c}$, where $B_{i}\left(\vec{s}_{i}\right)$ is very small and can be neglected. A perturbative calculation in second order of equation 2.15) reveals the cavity equations for $\vec{h}_{0}$ and $A_{0}$. This is achieved by attaching a bookkeeping factor $\lambda$ to $\vec{h}_{i}$ and $\vec{h}_{0}$ and a factor $\lambda^{2}$ to the matrices $A_{i}$ and $A_{0}$. As in the previous section, the cavity equations are found by calculating the spin expectation value $\left\langle\vec{s}_{0}\right\rangle_{c}$ from both the cavity partition function before merging, equation (2.6), and a partition function after merging (which is, in terms of $\vec{h}_{0}$ and $A_{0}$, Tre $e^{\beta \vec{h}_{0} \vec{s}_{0}+\beta \overrightarrow{s 0}^{T} A_{0} \vec{s}_{0}}$ ). To do so, we use the limit of the saddle point $z_{i}^{0}$ for $\vec{h}_{i} \rightarrow 0, z_{i}^{00}$. Up to second order in $\lambda$ we find

$$
\begin{aligned}
\vec{h}_{0} & =\sum_{i=1}^{k} \frac{\beta J_{i} \vec{h}_{i}}{2 z_{i}^{00}} \\
A_{0} & =\sum_{i=1}^{k}\left(\frac{\beta^{2} J_{i}^{2}}{4\left(z_{i}^{00}\right)^{2}} A_{i}-\frac{\beta^{3} J_{i}^{2}}{4 m\left(z_{i}^{00}\right)^{2} \sqrt{1+4 \beta^{2} J_{i}^{2}}} \vec{h}_{i} \otimes \vec{h}_{i}\right),
\end{aligned}
$$

where the symbol $\otimes$ denotes the tensor product. In first order (equation 2.16) we reproduce the result from above, the small field expansion in first order of equation (2.7). In second order (equation (2.17)), there is an explicit dependence of $A_{0}$ (after merging) on the effective fields $\vec{h}_{i}$ before merging. We note that these equations are still of a perturbative character. Up to second order, we do not find self-consistency of the cavity equations, which would have meant to find the cavity partition function of the form $\operatorname{Tr}_{\vec{s}_{0}} e^{\beta \vec{h} \vec{s}_{0}+\beta \vec{s}_{0}^{T} A \vec{s}_{0}}$. The cavity equations should become self-consistent for higher orders, probably a description of the cavity fields to infinite order would be necessary.

To find the critical temperature up to second order, we have to analyze the common distribution $Q(A, \vec{h})$, instead of $Q(\vec{h})$ in the previous section

$$
\begin{aligned}
Q(A, \vec{h})=E_{J} \int & \left(\prod_{i=1}^{k} d^{m \times m} A_{i} Q\left(A_{i}\right) d^{m} h_{i} Q\left(\vec{h}_{i}\right)\right) \\
& \delta\left(\vec{h}-\vec{h}_{0}\left(\left\{J_{i}\right\},\left\{\vec{h}_{i}\right\}\right)\right) \delta\left(A-A_{0}\left(\left\{J_{i}\right\},\left\{\vec{h}_{i}\right\},\left\{A_{i}\right\}\right)\right),
\end{aligned}
$$

where $\vec{h}_{0}\left(\left\{J_{i}\right\},\left\{\vec{h}_{i}\right\}\right)$ and $A_{0}\left(\left\{J_{i}\right\},\left\{\vec{h}_{i}\right\},\left\{A_{i}\right\}\right)$ are the solutions of equation 2.16), resp. equation (2.17). In order to find the instability of the trivial high temperature solution solely emerging from the matrices, we compute one iteration step of $Q_{A}(A)=E_{J} \int\left(\prod_{i} d^{m \times m} A_{i} Q\left(A_{i}\right)\right) \delta\left(A-A_{0}\left(\left\{J_{i}\right\},\left\{\vec{h}_{i}\right\},\left\{A_{i}\right\}\right)\right)$, similar to equation (2.8). This is not the true distribution of matrices, as it should depend on the vector fields $\vec{h}_{i}$ (from which follows $Q(A)=$ 
$\left.\int d h_{i}^{m} Q(A, \vec{h})\right)$, but exhibits the transition temperature $T_{c}^{A}$ in case the matrices would not depend on the vector fields. If $T_{C}^{A}$ is higher than $T_{c}$, there would be a second phase transition indicated by non-zero values of the matrices $A_{i}$, but if it is lower, the transition of the effective fields will determine the transition of the matrices and probably of all higher orders. This corresponds to a single, common critical temperature. The calculation is similar to the one of the previous section. The distribution of the entries of the cavity matrices before the iteration step, $A_{i}^{\alpha \beta}$ is assumed to be Gaussian with a small variance $\epsilon_{A}$. After the iteration step, the variance of the resulting distribution is $\epsilon_{A}^{\prime}$ and depends on the temperature. This yields the instability of the high temperature solution, which is the $\delta$-function around zero, at a critical temperature $T_{c}^{A}=\frac{J}{\sqrt[4]{k}}\left(1-\frac{1}{\sqrt{k}}\right)$. This temperature is lower than $T_{c}$ calculated solely from the cavity equation of the vector fields. As the instability of the matrix cavity equation is bound to the instability of the vector field cavity equation, there is a common transition temperature for the matrices $A_{i}$ and the vector fields $\vec{h}_{i}$. This is physically sensible and we expect this property to hold for all higher orders, if they were included in this calculation.

As the full formulation of this problem turns out to be necessary in order to describe the self-consistency of the cavity method for the whole low temperature phase, we have to regard the self-consistent effective field approach as the truncated model of this problem. It is correct in the vicinity of the phase transition and should yield the properties of the low temperature phase at least qualitatively. It therefore serves as the basis of the following sections of this chapter.

\subsection{Finite component numbers}

As equation 2.7 was derived using a saddle point approach, it only holds for $m \rightarrow \infty$. To analyse the problem for large, but finite component numbers, we have to introduce corrections around this saddle point. This is the starting point for investigating the breaking of replica symmetry, which we assume to happen for finite component numbers, as it does in the SK model, see Ref. [41. We will study this issue in chapter 4. Here we will show how to introduce corrections for finite component numbers for the implicit equation (2.7). To this end, we will implement these corrections to the saddle point in a power series in $\frac{1}{m}$ and take the first and second order corrections along. This changes equation (2.7) and shifts the critical temperature. With it, we have two equations at hand to study the phase transition numerically in the next sections and chapters, the one for $m \rightarrow \infty$ and the one for finite $m$.

The saddle point method for $m \rightarrow \infty$ was used to solve the $z$-integration 
in equation A.3. The technical details and how to introduce corrections to the saddle point and expand them in a power series in $\frac{1}{m}$ are described as Laplace's method in Ref. 68. There, the function $g(z)$ in the exponent of the integrand of the following integral which shall be evaluated by saddle point methods, is Taylor expanded around the saddle point at $z_{0}$, as (where for instance $g^{\prime \prime}\left(z_{0}\right)$ is the second derivative of $g(z)$ with respect to $z$, evaluated at the value $z_{0}$ )

$$
\int d z e^{m g(z)}=\int d z \exp \left\{m\left(g\left(z_{0}\right)+g^{\prime}\left(z_{0}\right)\left(z-z_{0}\right)+\frac{1}{2} g^{\prime \prime}\left(z-z_{0}\right)^{2}+\cdots\right)\right\}
$$

After expanding the exponential exp $m\left[\frac{1}{6} \frac{d^{3}}{d z^{3}} g\left(z_{0}\right)\left(z-z_{0}\right)^{3}+\frac{1}{24} \frac{d^{4}}{d z^{4}} g\left(z_{0}\right)(z-\right.$ $\left.\left.z_{0}\right)^{4}+\cdots\right]$ (not explicitly shown in equation (2.19) ) and solving Gaussian integrals for $\sqrt{m} z$, we sort the resulting terms according to their orders in a power series in $\frac{1}{m}$. The general result up to second order is

$$
\begin{aligned}
\frac{1}{m} \ln \int d z e^{m g(z)}=g\left(z_{0}\right) & +\frac{1}{m}\left(\frac{1}{2} \ln \left(\frac{2 \pi}{m}\right)-\ln \left(g^{\prime \prime}\left(z_{0}\right)\right)\right) \\
+ & \frac{1}{m^{2}}\left(\frac{1}{8} \frac{\frac{d^{4}}{d z^{4}} g\left(z_{0}\right)}{\left(g^{\prime \prime}\left(z_{0}\right)\right)^{2}}+\frac{5}{24} \frac{\left(\frac{d^{3}}{d z^{3}} g\left(z_{0}\right)\right)^{2}}{\left(g^{\prime \prime}\left(z_{0}\right)\right)^{3}}\right)+\cdots
\end{aligned}
$$

We now turn to the problem of introducing finite component numbers in the self-consistent effective field approach. To this end, we apply the technique from above to the partition function $\tilde{Z}$ of the spin $\vec{s}_{0}$ in a single cavity field $\vec{h}_{0}$ in equation A.3 and the following calculation of the expectation value $\left\langle\vec{s}_{0}\right\rangle_{c}$. We find

$$
\left\langle\vec{s}_{0}\right\rangle_{c}=\frac{\beta \vec{h}_{0}}{2 z_{0}} f\left(z_{0}\right)
$$

with $f(x)=1+\frac{1}{m} \frac{4 x-2}{(4 x-1)^{2}}-\frac{1}{m^{2}} \frac{864 x^{5}-1120 x^{4}+526 x^{3}-107 x^{2}-8 x}{8\left(2 x-\frac{1}{2}\right)^{7}}$. For the cavity partition function in equation A.6. corrections up to $\frac{1}{m^{2}}$ are introduced in the partition functions $Z_{i}$, analoguously to above. For the following saddle point integration for $\vec{s}_{0}$ we do not need corrections to the saddle point in $\frac{1}{m}$, as we precisely find the spin expectation value $\left\langle\vec{s}_{0}\right\rangle_{c}=\frac{\int d y d s_{0}^{m} \vec{s}_{0} e^{m g\left(s_{0}\right)}}{\int d y d s_{0}^{m} e^{m g\left(s_{0}\right)}}$ to be the saddle point. This expectation value is the quantity of interest. Instead 
of equation $\mathrm{A} .9$ and equation $\mathrm{A} .8 \mathrm{n}$ we find

$$
\begin{aligned}
z & =\frac{1}{2\left(1-\left\langle\overrightarrow{s_{0}^{\prime}}\right\rangle_{c}^{2}\right)} \\
2 z\left\langle\vec{s}_{0}\right\rangle_{c} & =\sum_{i=1}^{k} \frac{\beta^{2} J_{i} \vec{h}_{i}}{2 z_{i}^{0}} f\left(z_{i}^{0}\right) .
\end{aligned}
$$

We repeat the manipulations leading to the implicit equation for the effective field without corrections to the saddle points (2.7), and obtain

$$
\vec{h}_{0}=\frac{2 z_{0}}{f\left(z_{0}\right)}\left(1-\frac{\left\langle\vec{s}_{0}\right\rangle_{c}^{2}}{m}\right) \sum_{i=1}^{k} \frac{\beta J_{i} \vec{h}_{i}}{2 z_{i}^{0}} f\left(z_{i}^{0}\right),
$$

with $\left\langle\vec{s}_{0}\right\rangle_{c}$ and the function $f(x)$ from equation (2.21). Analoguously to the previous sections, the true local fields $\vec{H}_{i}$ are calculated by using $k+1$ effective fields instead of $k$ in equation (2.24)

$$
\vec{H}_{0}=\frac{2 z_{0}}{f\left(z_{0}\right)}\left(1-\frac{\left\langle\vec{s}_{0}\right\rangle_{c}^{2}}{m}\right) \sum_{i=1}^{k+1} \frac{\beta J_{i} \vec{h}_{i}}{2 z_{i}^{0}} f\left(z_{i}^{0}\right),
$$

A first observation after introducing corrections to the saddle point method in equation (2.24) is a shift of the critical temperature. To see this, we compute the corresponding higher orders of the inverse critical temperature perturbatively as $\beta_{c}=\beta_{c}^{0}+\frac{1}{m} \Delta \beta_{c}^{1}+\frac{1}{m^{2}} \Delta \beta_{c}^{2}$ (where $\beta_{c}^{0}$ is the solution of equation (2.11). The critical temperature $T_{c}$ in a power series in $\frac{1}{m}$ is found by expanding $\frac{1}{\beta_{c}}$, with $T_{c}^{0}=\frac{1}{\beta_{c}^{0}}=1-\frac{1}{k}$. The calculation of $\beta_{c}^{1}$ and $\beta_{c}^{2}$ follows the derivation of the phase transition in the previous section. For the $\pm J$-model we have the explicit solution in zeroth order, $\beta_{c}^{0}$, from equation 2.12 . The first order correction is found as $\Delta \beta_{c}^{1}=-\frac{2 k}{(k-1)(k+1)}$, which yields $\Delta T_{c}^{1}=\frac{2(k-1)}{k(k+1)}$. We see that $T_{c}^{0}>\left|T_{c}^{1}\right|$ for all connectivities $>1$. For $k=1$, the transition temperature remains zero, and in the limit $k \rightarrow \infty$, $\Delta T_{c}^{1}=0$. The second order correction can be calculated to $\Delta \beta_{c}^{2}=\frac{4 k^{2}(11 k+19)}{(k+1)^{4}}$, which leads to $\Delta T_{c}^{2}=-\frac{2(k-1)\left(7 k^{2}-23\right)}{(k+1)^{4}}$. Again, in the fully connected model $(k \rightarrow \infty)$ and for $k=1$ there is no shift of the critical temperature, which probably holds for all higher orders.

\subsection{Configurations of effective fields}

It is not possible to calculate the distribution of effective fields analytically. Therefore we are restricted to analyze it numerically. The cavity equations (2.7) (saddle point approach) and (2.24) (with corrections to the saddle 
point up to $\frac{1}{m^{2}}$ ) are appropriate to simulate the effective fields $\vec{h}_{i}$ on a given Bethe lattice of connectivity $k+1$. For this purpose we calculate each of the $N(k+1)$ effective fields as the solution of equation (2.7), resp. equation (2.24), and iterate this procedure until we reach a steady state, i.e. until convergence to a stable configuration. Replica symmetry corresponds to a single stable state the configuration of fields can be in. Hence, the iteration will converge to this state, as long as the spin glass is replica symmetric, which we expect to be the case for large $m$, in correspondence to the generalized Bose-Einstein condensation at $T=0$ discussed in the introduction. (For now, this is all we can say about the large- $m$ limit for finite temperatures. The dependence of replica symmetry on the number of components will be discussed in chapter 4). After convergence we use the resulting values of the effective cavity fields to calculate the true local fields $\vec{H}_{i}$, according to equation (2.9), resp. equation (2.25). Due to thermal fluctuations at finite temperatures each spin $\vec{s}_{i}$ fluctuates around its mean local field $\vec{H}_{i}$. Therefore, these fields determine the mean direction the spins point and we are able to investigate the space these fields span. Due to the invariance of rotation of vector spin glasses, each local field points in a different direction for different starting configurations of the spins and for different realisations of the Bethe lattice. This fact makes the algorithm we use the method of choice to observe the space of local fields of a single sample. We note that the basic idea of the algorithm is indeed different to a usual population dynamics algorithm [20]. There, no specific single lattice is selected, but one assumes to be in the thermodynamic limit where the distribution of effective fields is self-averaging. Here, we regard a finite number of spins on a single specific Bethe lattice and no disorder averaging takes place. Therefore, the results we will obtain (as for instance, the critical temperature and the space the local fields span) depend on the specific choice of disorder, i.e. on the selected Bethe lattice. It should be mentioned that the phase transition we find is indeed sharp, though the number of spins, $N$, is finite. The reason is that the number of components (though finite as well) corresponds to the limit $m \rightarrow \infty$. This is a characteristic of the large- $m$ limit. Due to that, the number of degrees of freedom is infinite and the phase transition is sharp.

The algorithm is sketched as follows and described in detail below:

1. Build a random Bethe lattice

2. Initialise the effective cavity fields with random values

3. Find new effective cavity fields $\vec{h}_{i j}$ for the whole lattice as the solution of the implicit equation (2.7), resp. 2.24 
4. Repeat the calculation of 3., until convergence to a stable state (criterion: convergence of the variance of the configuration)

5. Calculate the true local fields $\vec{H}_{i}$

The algorithm starts by choosing randomly a Bethe lattice configuration. This is done by selecting subsequently two different spins at random from a list of size $N(k+1)$ where $k+1$ entries correspond to the index of each spin $\vec{s}_{i}$ resp.. These two spins are connected by a link $J_{i j}$, taken from a Gaussian distribution. The chosen entries are deleted from the list afterwards. We do not allow to connect two spins twice to each other. When the list is empty, we have built a single random lattice with a fixed number of nearest neighbours, equal to $k+1$. With this given configuration we calculate the effective fields $\vec{h}_{i j}$, according to the equations (2.7) or 2.24). The notation $\vec{h}_{i j}$ denotes the effective field onto the spin $\vec{s}_{i}$, taking the links to all neighbouring spins into account except the link $J_{i j}$ to the spin $\vec{s}_{j}$. At first, we generate a random configuration of effective fields. Then, we iterate the implicit equations subsequently for each of the $N(k+1)$ effective fields $\vec{h}_{i j}$, keeping all other fields $\vec{h}_{i}$ fixed during the iteration. The iteration converges to a certain value of $\vec{h}_{i j}$. In that way, we renew each of the fields. After a single run we calculate mean, $\vec{h}=\frac{1}{2 N_{B}} \sum_{i=1}^{N} \sum_{j}^{k+1} \vec{h}_{i j}\left(N_{B}\right.$ is the number of bonds, which is $\left.\frac{N(k+1)}{2}\right)$, and variance, $(\Delta h)^{2}=\frac{1}{2 N_{B}} \sum_{i=1}^{N} \sum_{j}^{k+1}\left(\vec{h}_{i j}-\overrightarrow{\bar{h}}\right)^{2}$, of the new configuration of effective fields and check for convergence, which is assumed to be when the new variance is sufficiently close to the old variance. If convergence is reached, we calculate the true local fields $\vec{H}_{i}$ from equation $(2.9)$, resp. equation (2.25). The conjecture of finding a stable state when the absolute values of mean and variance of the configuration take stable values while iterating, is adapted from the analytic criterion of finding a stable distribution of fields, see equation (2.8). However, finding a stable mean and variance of a configuration of fields is not a sufficient criterion for a stable configuration itself, due to the invariance of the field configuration under rotation. It includes the possibility of a configuration which rotates in each single iteration step, but with stable absolute values of mean and variance.

In the forthcoming chapters we will regard different aspects of the configurations of effective fields. Here we will show that the algorithm indeed finds a phase transition in the large- $m$ limit (i.e. for large enough $m$ to be replica symmetric, as we will see in chapter 4). As described in section 2.1.1, the spin glass phase transition is indicated by a nonzero variance in the low temperature phase, while the mean remains zero, i.e. the fields become frozen into directions, which are fixed in time, but still random in space according to the configuration of disorder. The situation is different if the phase transition is 
described by a rotating configuration of fields while iterating. In that case the fields are not frozen in time, though the variance is non-zero as well, such that we do not see any difference to a usual spin glass phase transition at first glance. We will analyze this possibility in the following chapter. With the criterion of a non-zero variance indicating the phase transition, we find a critical temperature close to the one calculated in table 2.1. Though the system is finite, the transition for a given configuration of bonds is sharp, in that the high temperature solution, $\delta\left(\vec{h}_{i}\right)$, becomes unstable at a temperature, which depends on the configuration of bonds. In the figures 2.3 - 2.9, we find a transition for various system sizes and connectivities, including corrections in $\frac{1}{m^{2}}$. The inset in each of the figures 2.4 -2.10 is a double logarithmic plot of the order parameter against the difference between the inverse temperature and the inverse critical temperature, in order to evaluate critical scaling. The label in the inset plots gives the fitted value of the critical exponent $\beta^{\prime}$ of $q_{E A} \sim(\Delta h)^{2} \sim\left(\beta-\beta_{c}\right)^{\beta^{\prime}}$. We find that the order parameter probably scales as $q_{E A} \sim(\Delta h)^{2} \sim\left(\beta-\beta_{c}\right)^{1}$, with the critical exponent $\beta^{\prime}$ corresponding to the mean field exponent of the Ising universality class [53].

The problem of the true nature of the observed transition will be discussed in the following chapters. For the time being, we state that we do find a phase transition in these models at a nonzero temperature (keeping in mind the pitfall of a rotating configuration).
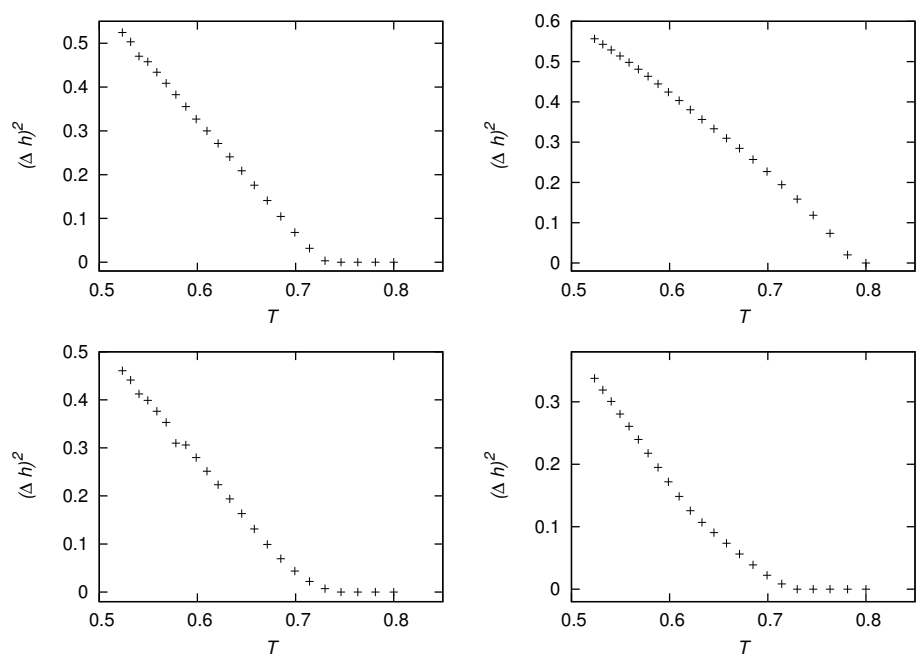

Figure 2.3: Results for 96 spins with 8 components on a Bethe lattice with connectivity equal to eight. The order parameter is plotted against the temperature for four different Bethe lattices. When $(\Delta h)^{2}$ becomes larger than zero, the phase transition happens. 


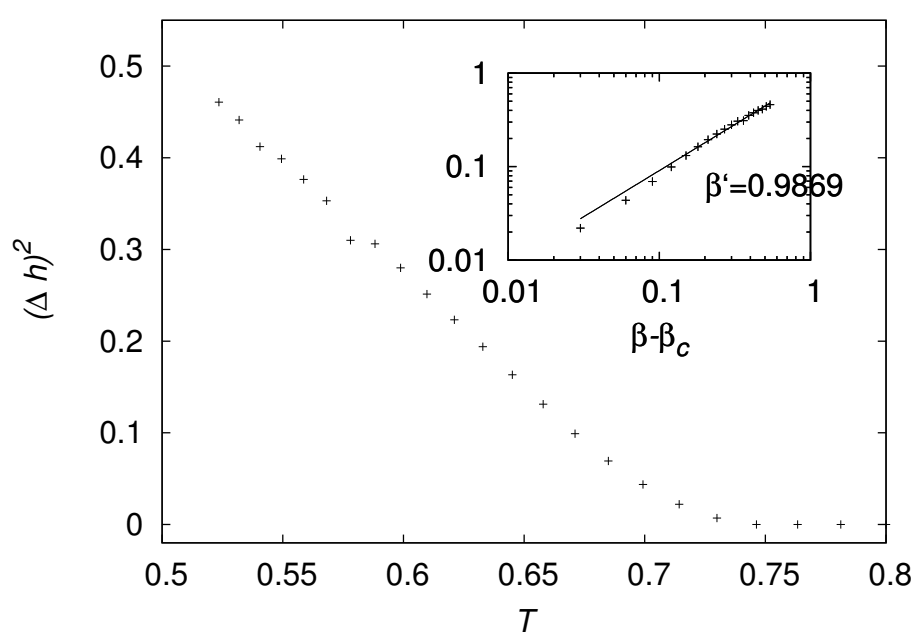

Figure 2.4: Critical scaling of the order parameter for 96 spins with 8 components on a Bethe lattice with connectivity equal to eight. The critical exponent is close to the 1 , the exponent of the Ising universality class.
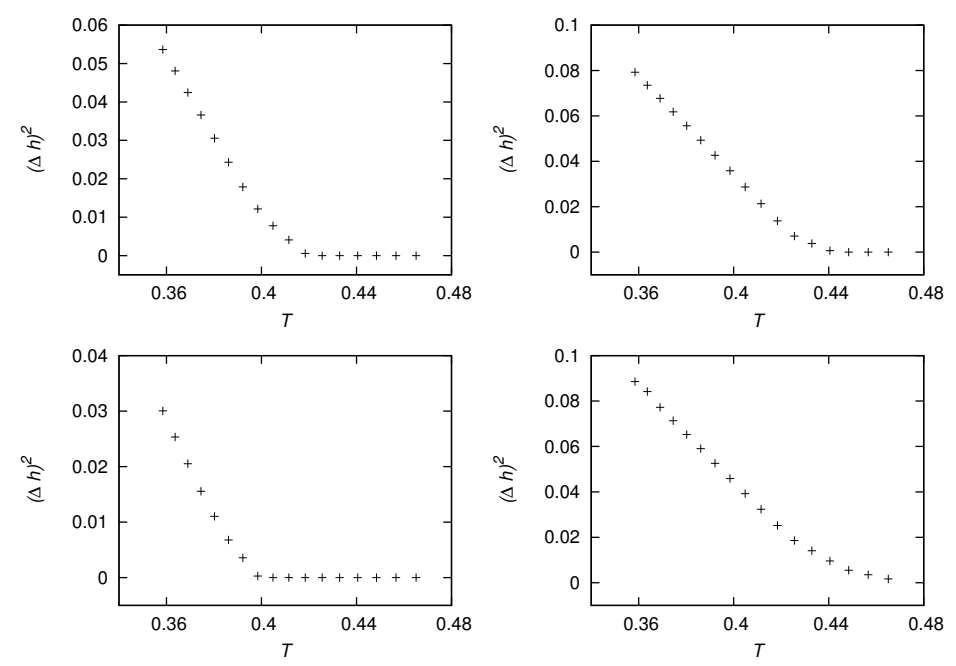

Figure 2.5: Phase transition for 256 spins with 8 components on four different Bethe lattices with connectivity equal to 4 . 


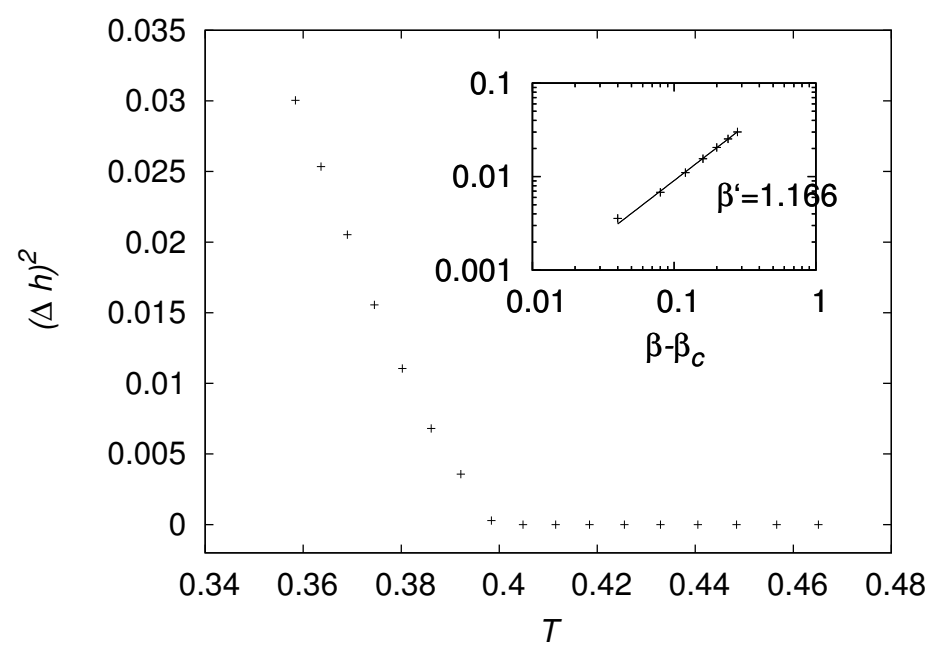

Figure 2.6: Critical scaling of the order parameter for 256 spins with 8 components on a Bethe lattice with connectivity equal 4. The critical exponent is comparable to that of the Ising universality class.
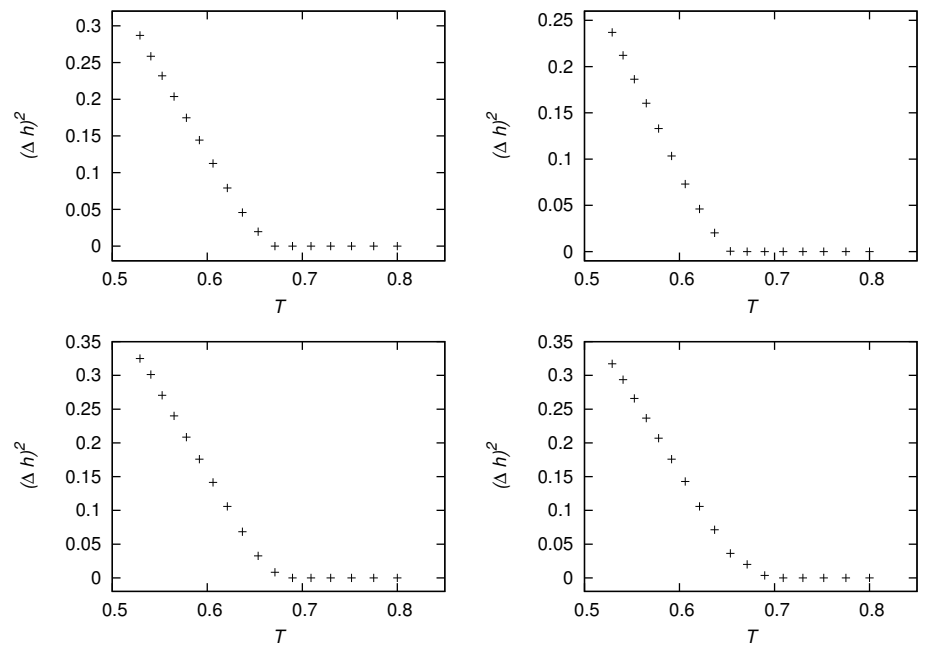

Figure 2.7: Phase transition for 256 spins with 8 components on four different Bethe lattices with connectivity equal to 8 . 


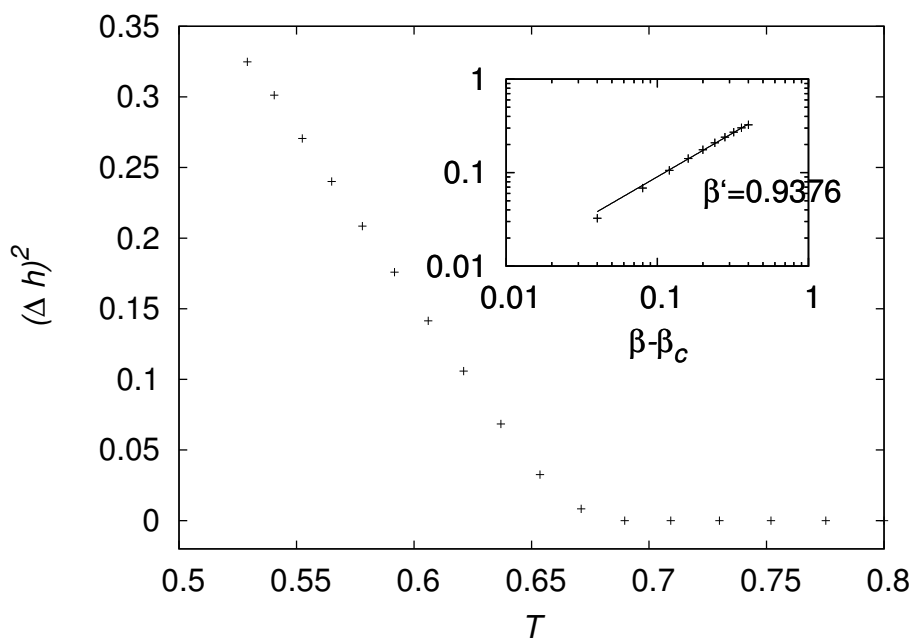

Figure 2.8: Critical scaling of the order parameter for 256 spins with 8 components on a Bethe lattice with connectivity equal 8. The critical exponent is close to 1 .
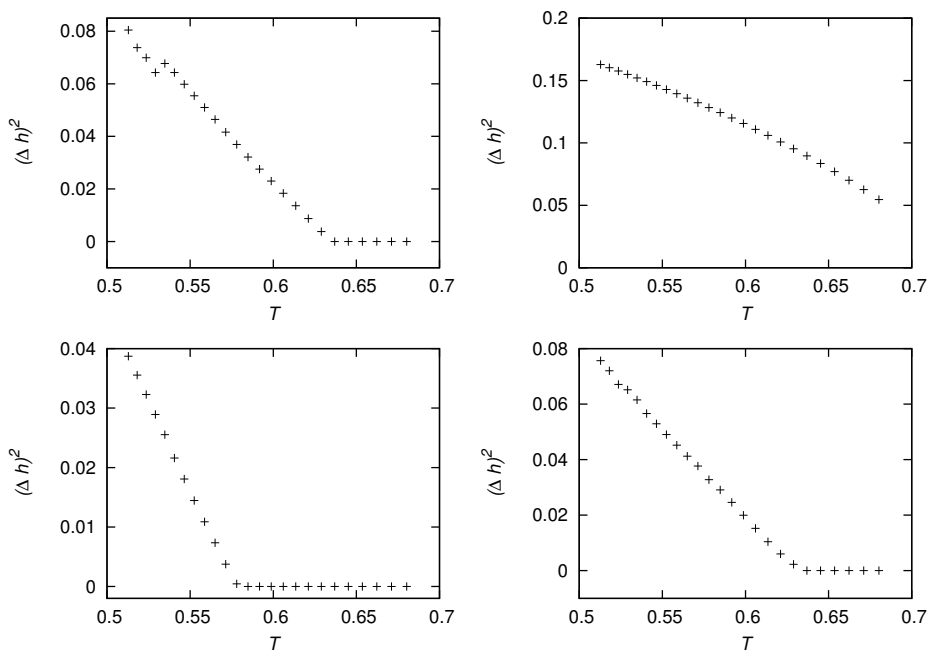

Figure 2.9: Phase transition for 512 spins with 4 components on four different Bethe lattices with connectivity equal to 6 . 


\subsection{PARALLEL TEMPERING MONTE CARLO ALGORITHM}

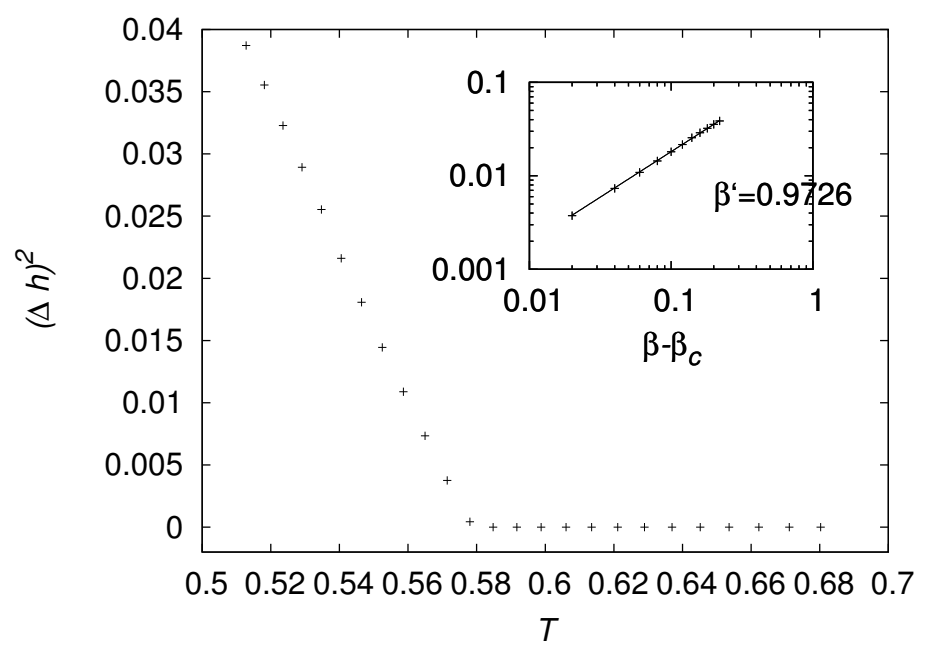

Figure 2.10: Critical scaling of the order parameter for 512 spins with 4 components on a Bethe lattice with connectivity equal 6. The critical exponent is close to 1 .

\subsection{Parallel Tempering Monte Carlo algorithm}

The parallel tempering Monte Carlo method has become a standard technique in statistical physics for analyzing disordered systems with a complicated free energy landscape such as spin glasses. It circumvents equilibrating a single system solely at one low temperature where it takes very long times to pass into different valleys of the free energy. Above the critical temperature the free energy barriers which separate the different valleys are not as hard to overcome as in the low temperature phase and the spin glass equilibrates fast. Thermal fluctuations dominate this temperature range and allow the spin glass to pass through the whole phase space. In other words, the spin glass is ergodic. On the other hand, below the critical temperature, the free energy landscape exhibits barriers, which are hard to overcome (in the thermodynamic limit of the SK-model they are infinitely high), such that the spin glass is easily trapped in a minimum (valley) in between two such energy barriers. A broken replica symmetry produces such a multi-valley structure, for which we find many stable equilibrium states and the phase space is broken into different ergodic regions. However, even for a replica symmetric system below $T_{c}$ (which we expect the $m$-component spin glass in the large- $m$ limit to be), the free energy landscape is complicated enough to impede the spin glass to pass through the whole phase space. In this section we use the parallel tempering Monte Carlo algorithm to calculate spin config- 
urations with low energy of two different copies $S_{1}$ and $S_{2}$ of a $m$-component spin glass with the same set of bonds, below the critical temperature. From each pair of these configurations we calculate the link overlap $q_{L}$ and analyze the probability distribution of $q_{L}$. In addition we will see that an appropriate order parameter can be derived by a slight extension of the link overlap. This method is capable of exhibiting the phase transition at $T_{c}$ and it does reveal the breaking of replica symmetry below $T_{c}$, which will be the subject of chapter 4 .

The parallel tempering Monte Carlo algorithm takes advantage of the ergodicity above $T_{c}$ in order to finally visit different valleys with the correct Boltzmann weight at temperatures below the critical temperature. In principle it consists of switching spin configurations of systems at different temperatures in such a way that every single system can overcome the free energy barriers, which separate the phase space, at high temperatures and then migrate down to lower temperatures and visit other valleys. To this end one takes several spin systems with the same set of bonds, here $N_{T}$ noninteracting copies of the same spin glass, which are simulated at different temperatures. Therefore each of the two copies $S_{1}$ and $S_{2}$ consists itself of a system of such $N_{T}$ copies of the same spin glass at different temperatures. Furthermore, due to averaging over the disorder, both copies $S_{1}$ and $S_{2}$ are simulated with a certain number of different configurations of bonds. The copies $1, \cdots, N_{T}$ are distributed over a set of temperatures $T_{1}, \ldots, T_{N_{T}}$, of which the lowest temperature $T_{1}$ is the temperature of interest and the highest temperature $T_{N_{T}}$ is sufficiently higher than the critical temperature. At high temperatures the spin system should be free to pass through the whole phase space and to adopt those states which it wouldn't have been able to reach from its current configuration of spins at the lowest temperature within an acceptable simulation time due to the free energy barriers. Though there may be quite many copies necessary to run it properly, the parallel tempering Monte Carlo algorithm speeds up simulations compared to running a simple Monte Carlo simulation of a single copy at $T_{1}$, by passing through the phase space of every single copy $1, \cdots, N_{T}$ and switching copies of neighbouring temperatures with an appropriate probability. Passing through the phase space is accomplished by allowing for a certain number of independent Monte Carlo moves of every single copy. A Monte Carlo move of a single copy chooses one of the $N$ spins of this copy and rotates this spin randomly into a new direction (keeping its length equal to $\sqrt{m}$ ). Denoting the initial configuration of spins with $\left\{\vec{s}_{i}\right\}$ and the final configuration, in which we substitute the original spin in favour of the rotated spin, with $\left\{\vec{s}_{f}\right\}$, we find two different values of the energy, the initial energy $E_{i}=E\left(\left\{\vec{s}_{i}\right\}\right)$ and the final energy $E_{f}=E\left(\left\{\vec{s}_{f}\right\}\right)$ (the energy is calculated according to $\left.-\sum_{\langle i j\rangle} J_{i j} \vec{s}_{i} \vec{s}_{j}\right)$. 
To decide whether the copy keeps the old configuration or takes the new configuration including the rotated spin instead of the original one, we use the Metropolis probability of accepting the new configuration $P\left(\left\{s_{i}\right\} \rightarrow\left\{s_{f}\right\}\right)$

$$
P\left(\left\{s_{i}\right\} \rightarrow\left\{s_{f}\right\}\right)=\left\{\begin{array}{l}
\exp \left(-\beta\left(E_{f}-E_{i}\right)\right) \quad \text { if } E_{f}-E_{i}>0 \\
1 \quad \text { otherwise }
\end{array} .\right.
$$

We see that if rotating the spin lowers the energy, i.e. $E_{f}-E_{i}<0$, the move is accepted with probability one. If it does not lower the energy, it is accepted according to the Boltzmann weight both configurations have. This probability satisfies the detailed balance condition

$$
Q_{i} P(i \rightarrow f)=Q_{f} P(f \rightarrow i)
$$

in that the transition probability of one state to the other, $P(i \rightarrow f)=$ $P\left(\left\{s_{i}\right\} \rightarrow\left\{s_{f}\right\}\right)$, times the probability of being in the original state $Q_{i}$ (which is proprotional to its Boltzmann weight $\exp \left(-\beta E_{i}\right)$ ) is equal to the probability of being in the other state, $Q_{f} \propto \exp \left(-\beta E_{f}\right)$, times the transition probability to the first state $P(f \rightarrow i)=P\left(\left\{s_{f}\right\} \rightarrow\left\{s_{i}\right\}\right)$. In the simulation, we repeat the attempt to lower the energy of every single copy (i.e. to let the system equilibrate at each temperature) $N$ times. In such a way, we let the copy find a new configuration, which increases the probability of passing to other valleys of the free energy at lower temperatures. To this end, we have to allow for swapping the temperatures of two copies at neighbouring temperatures $T_{i+1}$ and $T_{i}$ with $T_{i}<T_{i+1}$, resp. to switch the spin configurations. This is achieved by accepting such a swap with the probability $P_{T}\left(T_{i} \leftrightarrow T_{i+1}\right)$

$$
P_{T}\left(T_{i} \leftrightarrow T_{i+1}\right)=\left\{\begin{array}{ll}
\exp (-\Delta \beta \Delta E) & \text { if } \Delta \beta \Delta E>0 \\
1 & \text { otherwise }
\end{array},\right.
$$

where $\Delta E=E\left(\left\{\vec{s}_{T_{i+1}}\right\}\right)-E\left(\left\{\vec{s}_{T_{i}}\right\}\right)$ is the energy difference between both configurations and $\Delta \beta=\frac{1}{T_{i}}-\frac{1}{T_{i+1}}>0$ is the difference of the inverse temperatures. As above, the swap is accepted if the copy at $T_{i+1}$ produces a lower energy than the copy at $T_{i}$. Otherwise it is accepted according to the Boltzmann weight of both configurations. This acceptance rate satisfies the detailed balance condition as well. We see that the basic idea of a swap move is that the spin configuration at the higher temperature $T_{i+1}$ takes advantage of its less obstructive phase space to lower its energy in such a way, that the spin configuration of this low energy has a significant Boltzmann weight at $T_{i}$. 
By trying subsequently to swap the copies of each of two neighbouring temperatures, according to the probability $P_{T}\left(T_{i} \leftrightarrow T_{i+1}\right)$, each copy performs a random walk in the given temperature range. If, for instance, a copy is trapped at a certain valley at the lowest temperature, later on it will have been swapped to the highest temperature, where it is able to overcome the energy barriers easily and visit other valleys once it has been swapped to the lowest energy again. The copy at the highest temperature will migrate down to the lowest temperature if its spin configuration produces a sufficiently small energy (to be precise, every single system has the chance to lower its energy at its new temperature by a number of Monte Carlo moves as explained above. However, at lower temperatures it will not be able to pass through the whole phase space). If a copy visits the lowest temperature after having visited the highest one, we expect it to produce an independent configuration of spins with a low free energy at $T_{1}$. The number of parallel tempering Monte Carlo moves (swap trials) necessary for a copy to visit the lowest temperature twice while visiting the highest temperature in between is called the roundtrip time. We will measure this quantity in the starting phase of the simulations in order to store independent sets of spin configurations repeatedly after a number of parallel tempering Monte Carlo moves corresponding to the roundtrip time. Finally, we will be interested in certain properties of the link overlap distribution $P\left(q_{L}^{12}\right)$, of the link overlap $q_{L}^{12}=\frac{1}{N_{B}} \sum_{\langle i j\rangle}\left\langle\vec{s}_{i} \vec{s}_{j}\right\rangle_{1}\left\langle\vec{s}_{i} \vec{s}_{j}\right\rangle_{2}$ between the spin configurations with the same temperature of two copies $S_{1}$ and $S_{2}$ of the same spin glass with identical sets of bonds (where the index at the brackets $\langle\cdot\rangle$ denotes the thermal average with respect to only one of the two copies 1 and 2 and $N_{B}$ is the number of nearest neighbours, here $\left.\frac{N(k+1)}{2}\right)$. We see that the link overlap quantifies correlations between the two copies $S_{1}$ and $S_{2}$. Therefore we have to simulate these two copies, each with the same set of temperatures, simultaneously but independently and find the distribution $P\left(q_{L}^{12}\right)$ by averaging over the results of the simulation. The phase transition itself can not be observed from the link overlap distribution, as the link overlap takes only the correlations between neighbouring spins into account. However, the spin glass phase transition is indicated by long range order. That is why we have to take the overlap of each pair of spins into account and calculate the quantity $q^{2}=\frac{1}{N(N-1) m^{2}} \sum_{i, j}\left\langle\vec{s}_{i} \vec{s}_{j}\right\rangle_{1}\left\langle\vec{s}_{i} \vec{s}_{j}\right\rangle_{2}$, which is the mean correlation between each two of the $N$ spins.

In Ref. [47, the authors give an argument at $T=0$ why this quantity must vanish as a power law of the system size, as $N^{-\mu_{m}}$, with a connectivity dependent exponent $\mu_{m}$. Their line of argumentation is based on the phenomenon that the ground state spins use only components up to an upper bound, $m_{0}$, which scales with the system size as $N^{\mu_{m}}$ [45, 46, 47, 48]. The phenomenon 
of a reduced component number can therefore only be observed for finite system sizes. If the number of available components $m$ is larger than $m_{0}$, we call this the large- $m$ limit. The large- $m$ limit corresponds to taking the limit $m \rightarrow \infty$ before the thermodynamic limit (which restores replica symmetry according to Ref. [41]). It is due to this correspondence that it is assumed that the $m$-component spin glass is replica symmetric in the large- $m$ limit whereas replica symmetry is broken for component numbers smaller than $m_{0}$ (we will discuss this problem in chapter 4). The phenomenon of a reduced component number is called the generalized Bose-Einstein condensation [46] and we discuss it at $T=0$ and at finite temperatures in chapter 3 . For now, we simply assume that this phenomenon is not restricted to the ground state, but holds in the whole low temperature phase. The order parameter $q^{2}$ can be expressed as the average overlap $\left[\left\langle\vec{s}_{i} \vec{s}_{j}\right\rangle_{1}\left\langle\vec{s}_{i} \vec{s}_{j}\right\rangle_{2}\right]_{\text {av }}$. In case of replica symmetry we expect the equilibrium state for a given temperature to be the same for both copies 1 and 2 (up to a global rotation of all spins of each copy). Therefore the angle $\Theta$ between $\vec{s}_{i}$ and $\vec{s}_{j}$ should be the same in both copies. Furthermore, $\cos \Theta$ is the average of the (normalized) $z$-component $\left[s_{z}\right]_{\text {av }}$ of the spin $\vec{s}_{j}$, if we take the $z$-axis along $\vec{s}_{i}$ for both copies. Then we can express $q^{2}$ as $q^{2}=\left[\cos ^{2} \Theta\right]_{\mathrm{av}}=\frac{1}{{\overrightarrow{s_{j}}}^{2}}\left[s_{z}^{2}\right]_{\mathrm{av}}$. Using that the average $\left[s_{z}^{2}\right]_{\mathrm{av}}$ is approximately the same for all spin components $m_{0}$ the spins use in the large- $m$ limit, one finds $q^{2} \approx \frac{1}{m_{0}} \frac{\sum_{\alpha=1}^{m_{0}}\left[s_{\alpha}^{2}\right]_{\text {av }}}{{\overrightarrow{s_{j}}}^{2}}=\frac{1}{m_{0}} \sim N^{-\mu_{m}}$ (where we have used that the spins are normalized to $\sqrt{m}$ ). The authors in Ref. [47] call this observation "quasi-long-range order" as the correlations decay with a power of the system size, resp. with a power of distance for finitely connected lattices. This property is reminiscent of the Kosterlitz-Thouless transition of the two dimensional XY ferromagnet, where there is no long range order but a vanishing order parameter below the critical temperature [49, 53]. However, scaling the parameter $q^{2}$ with $m$ (which is the definition of the order parameter in Ref. [41]) gives a finite order parameter for $m>m_{0}$, as $m_{0}$ is at most equal to $m$. Therefore the phase transition is not of the KosterlitzThouless type in which there is no long range order, though both phenomena might have the same underlying explanation, Goldstone modes [53], which to find is beyond the scope of this thesis. However, in the following chapter we are going to discuss this relation in more detail.

There are two other settings to be made in the starting phase of the algorithm. On the one hand, generally in Monte Carlo simulations a system needs some time to equilibrate, i.e. to be in thermal equilibrium, when starting from a random configuration. We take care of this issue by a special test for equilibration. On the other hand, we have to choose an appropriate number of temperatures with an appropriate distance between them to speed 
up simulations. To achieve this, we have implemented a feedback algorithm, which chooses the distance between two temperatures in such a way to make the transition rate between them to be close to $\frac{1}{2}$, which seems a reasonable value to allow many transitions from the highest to the lowest temperature and vice versa.

In thermal equilibrium the spin configurations of both copies $S_{1}$ and $S_{2}$ satisfy the relation

$$
E_{J} q_{L}^{12}-E_{J} q_{s}=\frac{2}{(k+1)} T U
$$

at each temperature. It is valid for a Gaussian distribution of bonds and involves the average energy per spin $U=-E_{J} \sum_{\langle i j\rangle} J_{i j}\left\langle\vec{s}_{i} \vec{s}_{j}\right\rangle$, the link overlap $q_{L}^{12}=\frac{1}{N_{B}} \sum_{\langle i j\rangle}\left\langle\vec{s}_{i} \vec{s}_{j}\right\rangle_{1}\left\langle\vec{s}_{i} \vec{s}_{j}\right\rangle_{2}$ and the overlap $q_{s}=\frac{1}{N_{B}} \sum_{\langle i j\rangle}\left\langle\vec{s}_{i} \vec{s}_{j}\right\rangle^{2}$. Each of the sums is taken over all pairs of nearest neighbours $(\langle i j\rangle)$ and $\left\langle\vec{s}_{i} \vec{s}_{j}\right\rangle$ denotes the thermal average. The relation $(2.29)$ is derived by integrating by parts the expression for the average energy with respect to the bonds $J_{i j}$, which are taken to be independent Gaussian random variables [34]. Measuring each of those quantities at $T_{1}$ during the equilibration phase makes the left and right hand side of equation 2.29 approach the same value. Therefore, we have to simulate a certain number of different lattices (different configurations of disorder of $S_{1}$ and $\left.S_{2}\right)$ in order to average over the disorder $\left(E_{J}\right)$ in equation (2.29). In the beginning the right hand side is close to zero, as we initialize the spins in random directions. However, the left hand side will be too low in the beginning, as the link overlap between both copies $S_{1}$ and $S_{2}$ at the lowest temperature will be small due to the random initialisation and the overlap $q_{s}$ depends less on the Monte Carlo moves (for the Ising case it is simply a constant, equal to one. In case of $m$-component vector spins, it can be shown that $\vec{s}_{i} \vec{s}_{j}$ of a random initial spin configuration is equal to $\sqrt{m}$ on average. To see this, we regard the initial random configuration of spins. As each spin has a fixed length equal to $\sqrt{m}$, it has on average an entry of one in each single component. If we transform both spins $\vec{s}_{i}$ and $\vec{s}_{j}$ into a basis in which $\vec{s}_{i}$ is orientated along the $z$-axis, the spin $\vec{s}_{j}$ has an entry of one along this axis on average. With $\left|\vec{s}_{i}\right|=\sqrt{m}$ we find $\left\langle\vec{s}_{i} \vec{s}_{j}\right\rangle^{2} \approx m$, which leads to $q_{s} \approx m$ ). We expect the system to be in thermal equilibrium when both the right and left hand side come sufficiently close to each other and fluctuations are small.

The feedback algorithm starts by distributing the $N_{T}$ temperatures uniformly over the temperature range. In between two tests for equilibration of the whole spin glass system we need a sufficiently large number of parallel tempering Monte Carlo moves for proper averaging. This allows for measuring the transisition rate between each of two neighbouring temperatures, 
as $p_{i}=\frac{\text { accepted swap trials }(i+1 \rightarrow i)}{\text { total swap trials }(i+1 \rightarrow i)}$ for every temperature $T_{i}=T_{1}, \ldots, T_{N}-1$ within such single equilibration interval (A swap trial for $T_{i}$ is defined as the trial to switch it with the higher temperature $\left.T_{i+1}\right)$. We suppose that a transition rate of $p_{i} \approx \frac{1}{2}$ allows for an appropriate migration from the lowest to the highest temperature and vice versa. Therefore, we adjust the inverse temperatures after each equilibration test according to

$$
\beta_{i}^{\text {new }}=\beta_{i-1}+2 p_{i-1}\left(\beta_{i}^{\text {old }}-\beta_{i-1}^{\text {old }}\right),
$$

for $i=2, \ldots, N-1$, while $\beta_{1}=\frac{1}{T_{1}}$ and $\beta_{N}=\frac{1}{T_{N}}$ remain unchanged. The feedback algorithm (2.30) includes the transition rate found during the previous equilibration run. After repeatedly adjusting the temperatures, we reach a steady state in which the temperatures vary only little. It is crucial to choose the number of temperatures in such a way as to cover the temperature range properly. A general result is that the distance between two temperatures $\delta T$ scales with the inverse square root of the number of degrees of freedom, i.e. in the case of $m$-component vector spins as $\delta T \sim \frac{1}{\sqrt{N m}}$ [34]. We see that many more temperatures are needed for vector spin glasses than for the Ising spin glass, as the number of necessary components in the large- $m$ limit scales with the system size, which limits the simulations to rather small system sizes. Nevertheless, here we choose the number of temperatures such that the transition rates between neighbouring temperatures come close to $\frac{1}{2}$ (according to equation (2.30) and that the transition rate $p_{N_{T}-1}$ from the highest temperature to its neighbouring temperature is larger than $\frac{1}{2}$. The resulting set of temperatures enters the parallel tempering Monte Carlo algorithm, which calculates independent configurations of spins that minimize the energy at $T_{1}$.

After the equilibration phase in which we find a proper set of temperatures, the algorithm measures the roundtrip time of each single system with a different configuration of bonds and produces independent configurations of spins of both copies $S_{1}$ and $S_{2}$ at each temperature. The configurations are assumed to be independent, as we wait for a number of parallel tempering Monte Carlo moves corresponding to the measured roundtrip time before storing the following configuration of spins. From each two configurations of $S_{1}$ and $S_{2}$ at each temperature and for each configuration of disorder, we calculate the link overlap $q_{L}^{12}$ and the order parameter $q^{2}$. We have simulated systems from $N=48$ up to $N=1024$ (for $m=2$ ) in the temperature range of $[0.5: 1.5]$, and computed the overlaps at least 128 times. From the resulting data, we calculate histograms of the probability of observing a certain link overlap $P\left(q_{L}\right)$, which includes the thermal and the disorder averaging (i.e. averaging over the independent sets of spins and over all configurations 

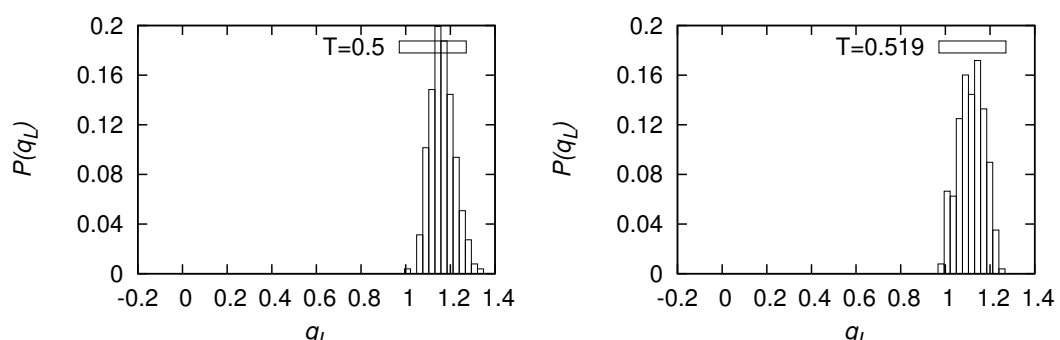

$q_{L}$
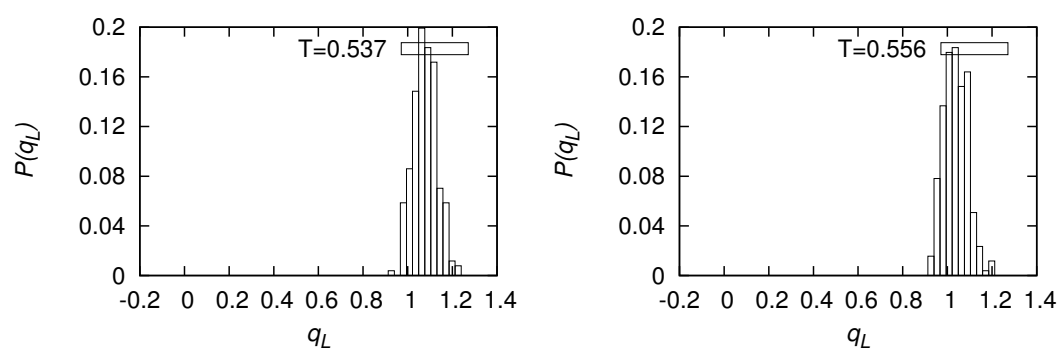

Figure 2.11: Histogram of the probability distribution of the link overlap for the four lowest temperatures simulated.

of disorder). These are presented in Figures 2.11-2.15 for 256 spins with 6 components on a Bethe lattice with a connectivity of 8 . We show the histograms for the four lowest and the four highest temperatures and for twelve temperatures in between (in total we have simulated 29 temperatures for this system size). Their properties are characteristic for the resulting histograms of all other system sizes. At all temperatures they get peaked around a typical mean value $\left[q_{L}\right]$ (which can be larger than one, due to not normalizing $q_{L}$ with respect to $m$ ). Further, from the bare shape of the histograms it is not possible to deduce a phase transition, nor is it possible to deduce the breaking of replica symmetry.

The reason that we do not see the phase transition is due to the finite size of the system, as a sharp phase transition can only occur in the thermodynamic limit (resp. for an infinite number of degrees of freedom). In finite systems the phase transition is smeared out. However, the quantity $q^{2}=\frac{1}{N(N-1)} \sum_{i, j}\left\langle\vec{s}_{i} \vec{s}_{j}\right\rangle_{1}\left\langle\vec{s}_{i} \vec{s}_{j}\right\rangle_{2}$ is an appropriate order parameter to observe the phase transition by finite size scaling methods. It should scale as $N^{-\mu_{m}}$ in the low temperature phase, where $\mu_{m} \approx .377$ for the Bethe lattice with a connectivity of eight [48]. The scaling ansatz for $q^{2}$ depends on the system size and the distance between the simulated temperature $T$ and the critical temperature $T_{c}$, which we expect at about 0.7 , see table 2.1. The scaling 

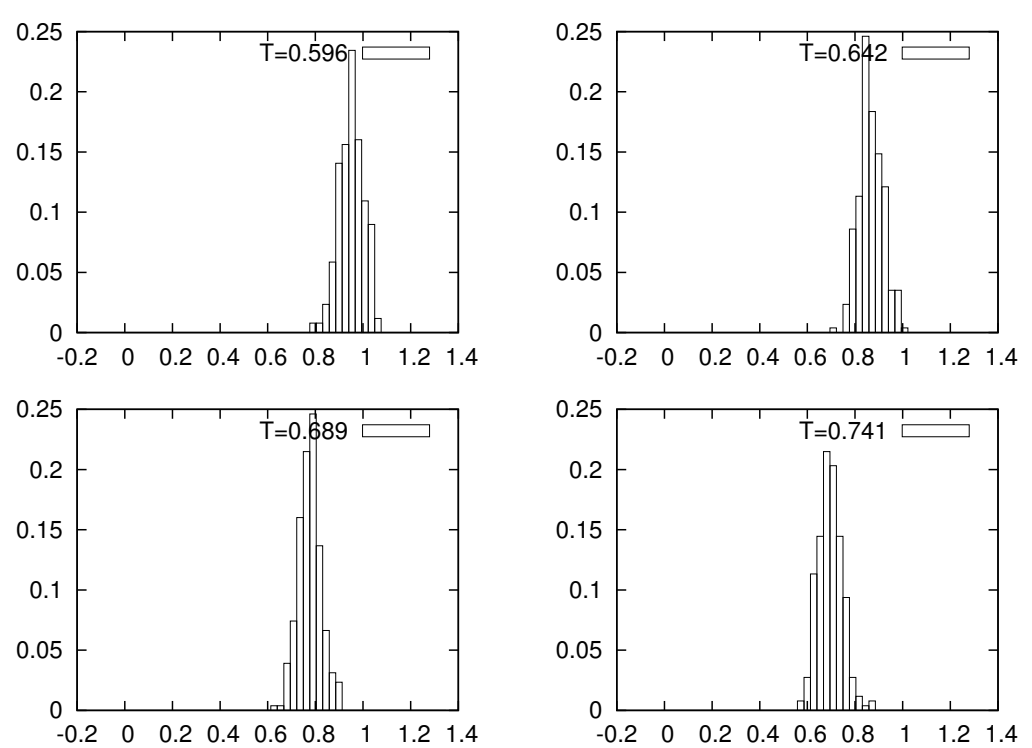

Figure 2.12: Histogram of the probability distribution of the link overlap for four temperatures covering a range including $T_{c}$.
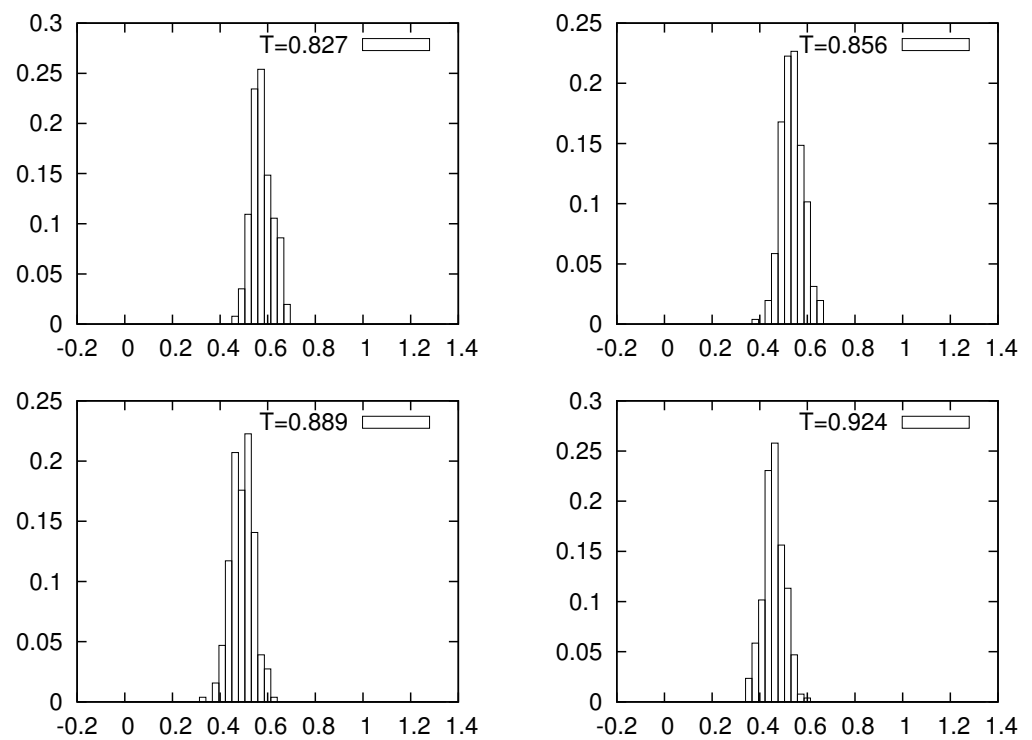

Figure 2.13: Histogram of the probability distribution of the link overlap for four temperatures above $T_{c}$. 

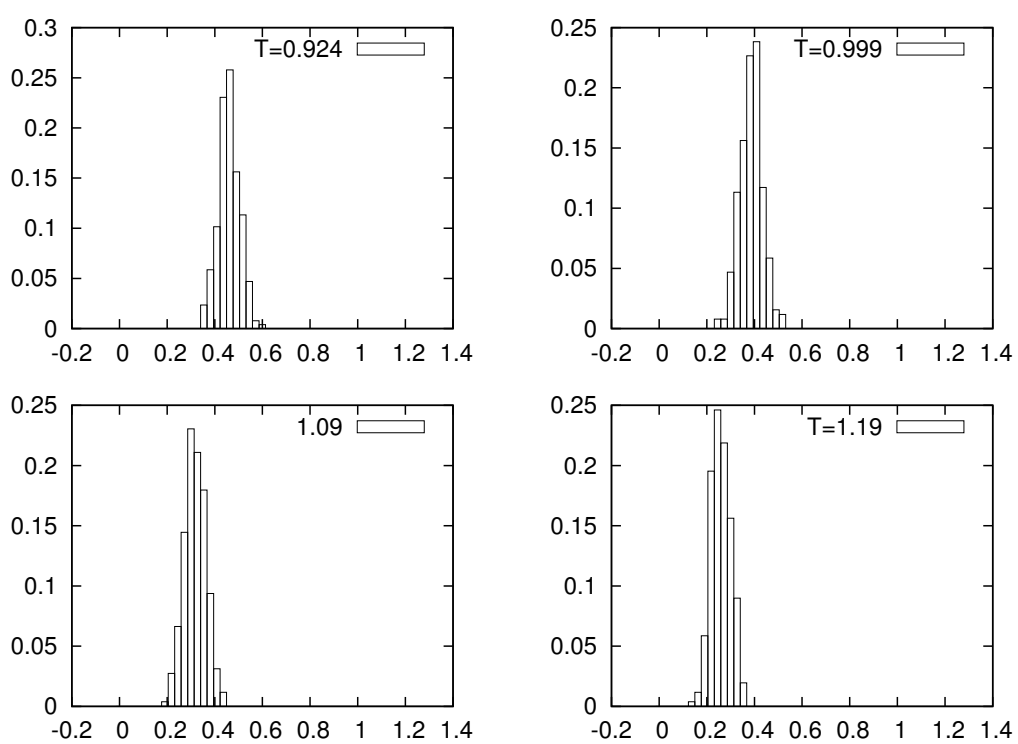

Figure 2.14: Histogram of the probability distribution of the link overlap for four temperatures above $T_{c}$.
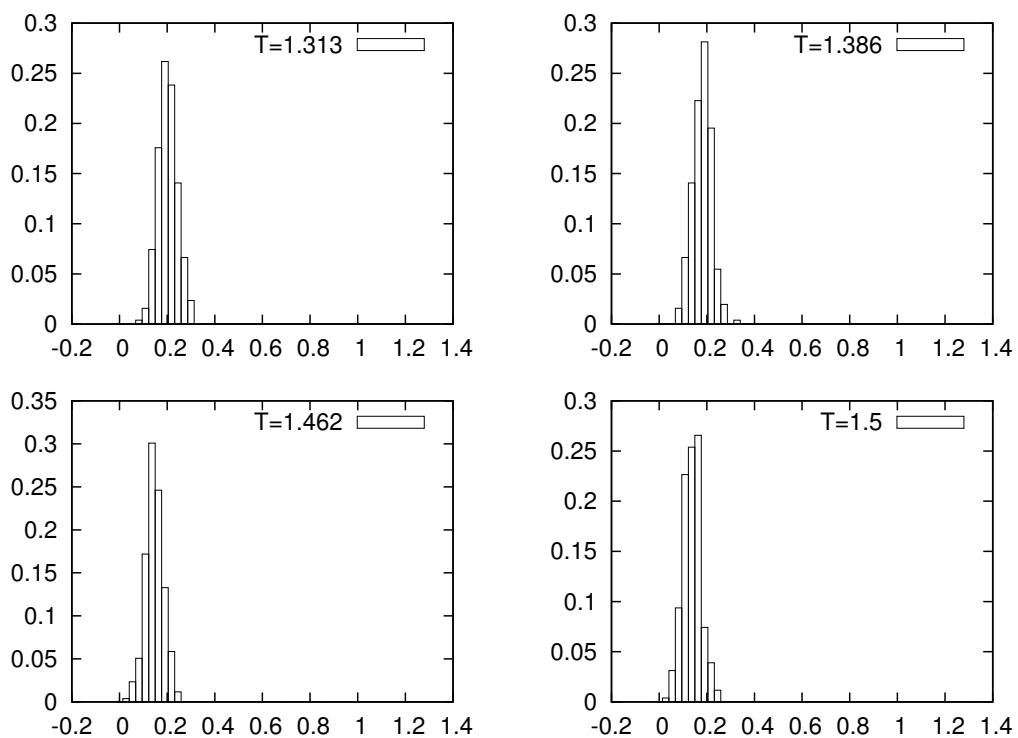

Figure 2.15: Histogram of the probability distribution of the link overlap for the four highest temperatures. 


\subsection{PARALLEL TEMPERING MONTE CARLO ALGORITHM}

ansatz therefore is

$$
q^{2}=N^{-\alpha^{\prime}} \mathcal{F}\left(\left(T-T_{c}\right) N^{\beta^{\prime}}\right),
$$

where the scaling function $\mathcal{F}(x)$ scales as $|x|^{\gamma^{\prime}}$ for $x \rightarrow-\infty$ (i.e. for small $T$ ). The scaling exponents therefore should satisfy the relation $\alpha^{\prime}-\beta^{\prime} \gamma^{\prime}=\mu_{m}$. If $q^{2}$ depends on only the two quantities $N$ and $T-T_{c}$, the data for different $N$ and $T$ should collapse onto a single function, when choosing $\alpha^{\prime}, \beta^{\prime}$ and $T_{c}$ appropriately. This means that the data should become independent of $N$ in the scaling plot. The phase transition is indicated by a fundamentally different scaling of $\mathcal{F}(x)$ for $x$ smaller, resp. larger than 0 (i.e. above and below the critical temperature), which we suggest as $\mathcal{F}(x) \rightarrow|x|^{\gamma^{\prime}}$ for $x \rightarrow-\infty$ and a decay as $\mathcal{F}(x) \rightarrow x^{-\gamma^{\prime \prime}}$ above $T_{c}$ (for $x \rightarrow \infty$ ). To investigate a larger temperature range, we have extended the simulated range of temperatures in the parallel tempering Monte Carlo simulations partially to [0.5 : 10.0], but did not observe a different behaviour as for the original temperature range of $[0.5,1.5]$. We indeed find critical finite size scaling for different components numbers $m$ from 2 to 6 , see figures 2.16, 2.18, 2.20 and 2.22. The parameter in these plots are $\alpha^{\prime}=0.6, \beta^{\prime}=0.2$ and $T_{c}=0.65$ and the data match very well. To support these findings, we check whether the scaling exponents satisfy the relation $\alpha^{\prime}-\beta^{\prime} \gamma^{\prime}=\mu_{m}$, which should be true according to the line of argumentation from Ref. [47] sketched above. To evaluate $\gamma^{\prime}$ we have plotted the data in the low temperature phase in a double logarithmic plot in figures 2.17, 2.19, 2.21 and 2.23, and deduce a common estimate of $\gamma^{\prime} \approx 1.1$, which leads to $\alpha^{\prime}-\beta^{\prime} \gamma^{\prime}=0.38$, very close to $\mu_{m}$ from Ref. [48]. Furthermore we find $\gamma^{\prime \prime} \approx 1.8$ (data not shown). In total, this is a strong indication of a phase transition at a critical temperature close (but slightly lower) to the one indicated by the simulation of a configuration of effective fields in the previous section (see figure 2.7). As we can resolve the problem of a vanishing order parameter $q^{2}$ by multiplying with $m$, we expect the transition to have freezing in disorder below $T_{c}$, even though the scaling property of $q^{2} \sim N^{-\mu_{m}}$ is reminiscent of a Kosterlitz-Thouless transition. The question whether the $m$-component spin glass has a phase transition with the spins freezing in time will be investigated in the following chapter.

In this section, the parallel tempering Monte Carlo algorithm has proved to be a very useful technique for vector spin glasses on Bethe lattices as well. We have been able to simulate spin systems in such a way to discover characteristic properties of the probability distribution of the link overlap. On the one hand, we have calculated histograms of the link overlap distribution. On the other hand, we investigated in detail whether the spin glass phase transition can be observed and used finite size scaling to support the finding of 


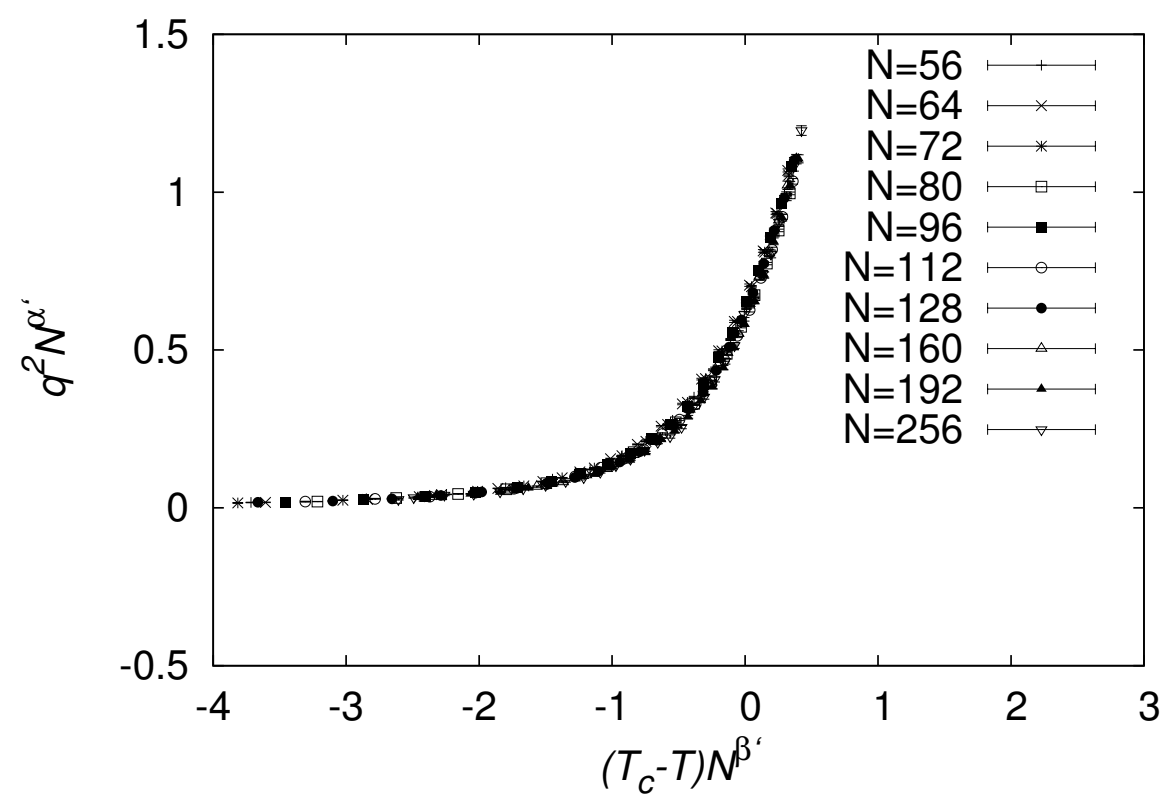

Figure 2.16: Scaling plot of the order parameter $q^{2}$ for a connectivity of eight and six components. See text for explanation.

a spin glass phase transition from sections 2.1.1 and 2.3, apparently slightly below $T_{c}$ from table 2.1. This further argument for a phase transition is very important, as the transition from the investigations of the configurations of effective cavity fields show some peculiar properties, as we will see in the next chapter. For now, the most convincing argument is that both methods almost coincide at the value of $T_{c}$.

\subsection{The Bethe lattice free energy}

Generally, it is a worthwhile task to study the free energy of any given system in statistical physics. Though there is no direct physical application of the result of this section, it might be helpful for future studies of the $\mathrm{m}$ component spin glass on a Bethe lattice to have the calculation of the free energy at hand.

The free energy on a Bethe lattice can be expressed in terms of the configuration of cavity fields [20]. In this section we present the calculation of the free energy for the $m$-component spin glass, not averaged over the disorder, but depending on a single configuration of effective fields, i.e. for a single set of bonds. 


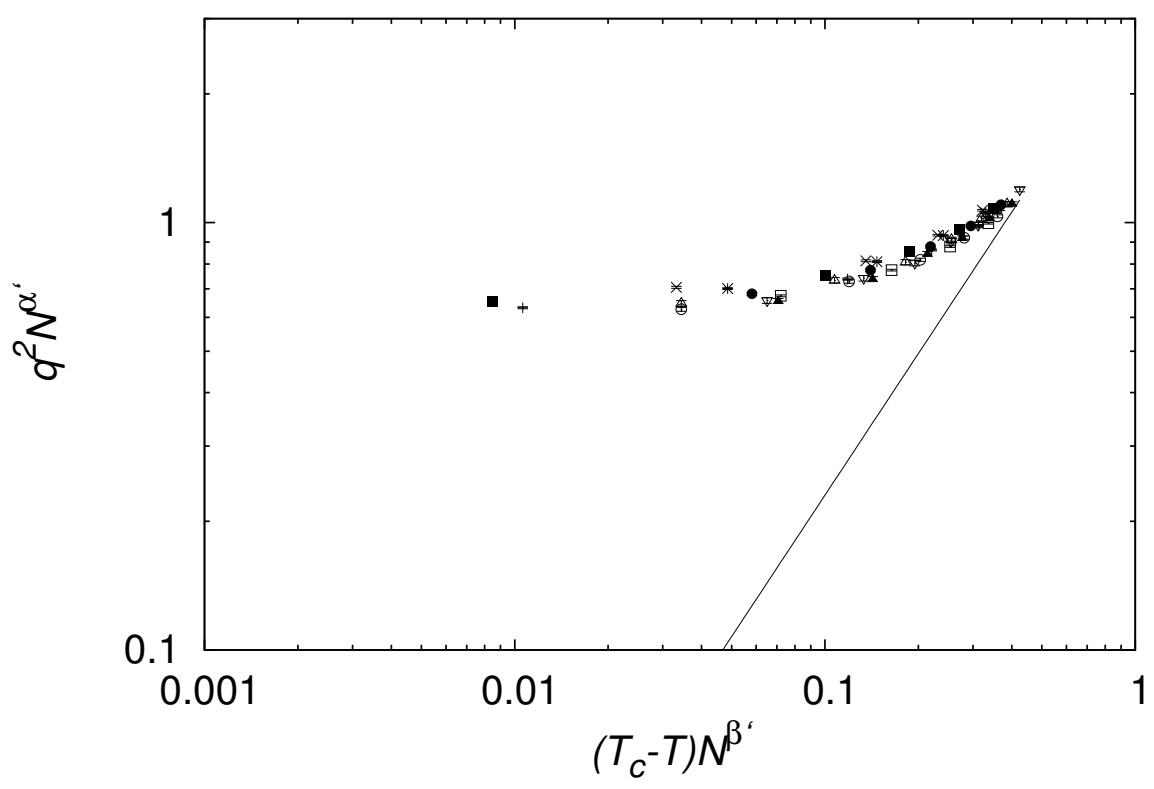

Figure 2.17: Double logarithmic scaling plot of $q^{2}$ in the low temperature phase with $k+1=8$ and $m=6$. The scaling function matches a scaling of $|x|^{\gamma^{\prime}}$ with $\gamma^{\prime} \approx 1.1$ (additionally plotted).

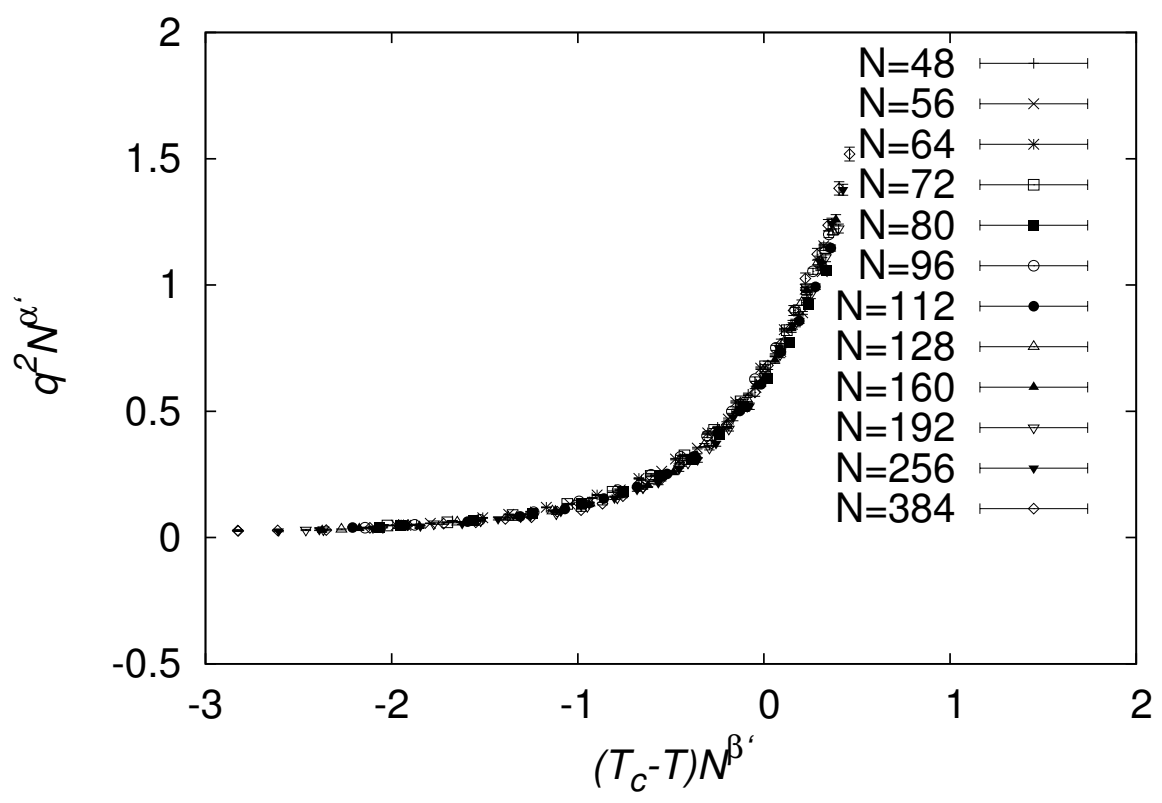

Figure 2.18: Scaling plot of $q^{2}$, with $k+1=8$ and $m=4$. 


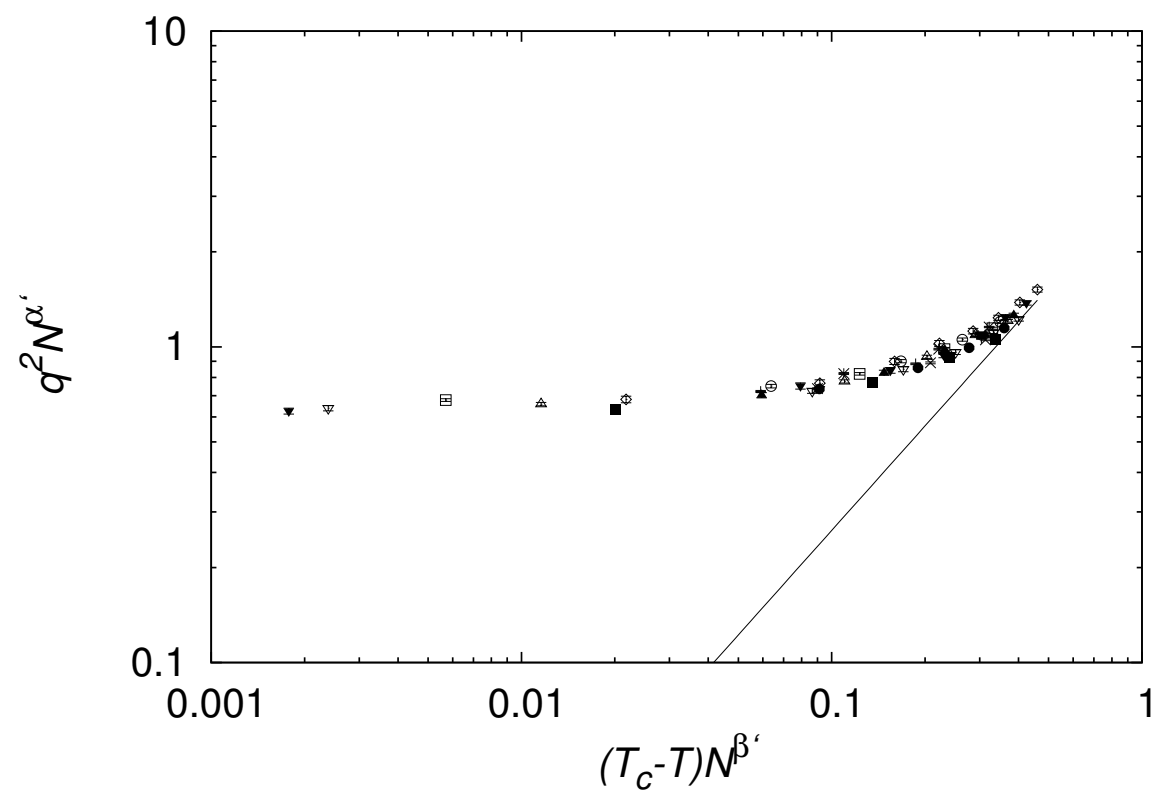

Figure 2.19: Double logarithmic scaling plot of $q^{2}$ with $k+1=8$ and $m=4$. Additionally plotted: $|x|^{1.1}$

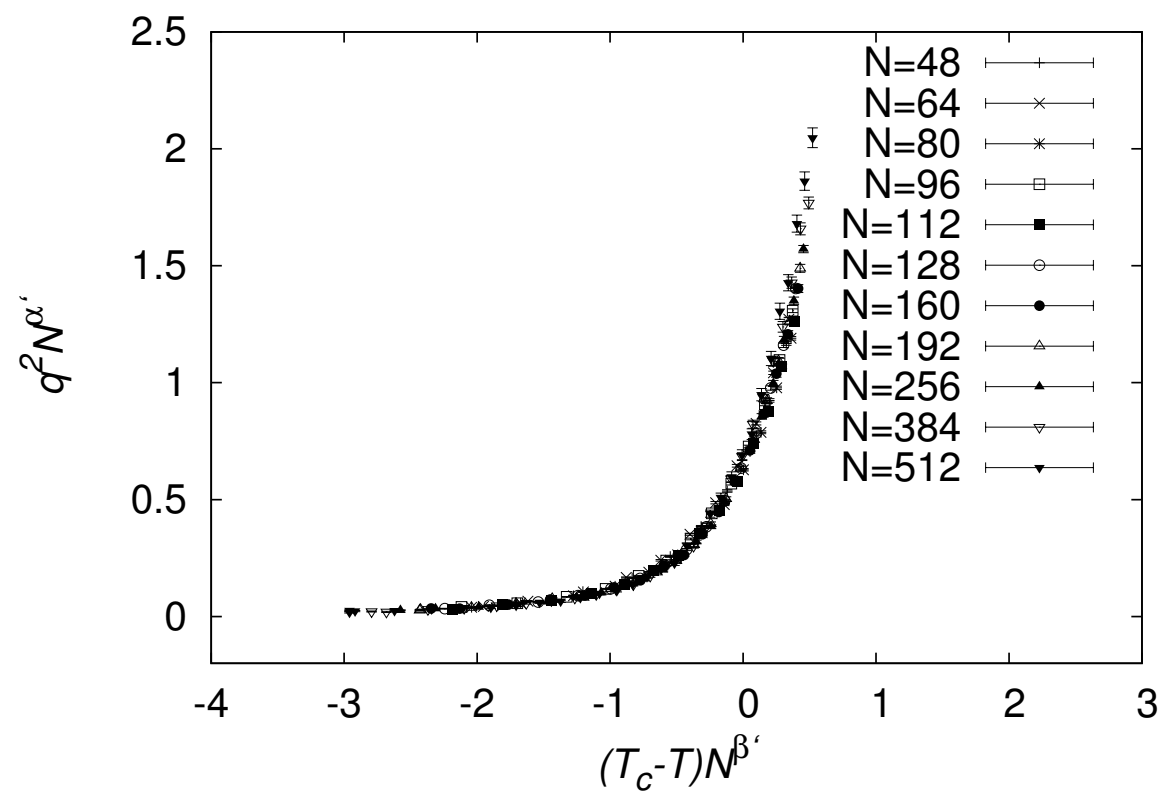

Figure 2.20: Scaling plot of $q^{2}$, with $k+1=8$ and $m=3$. 


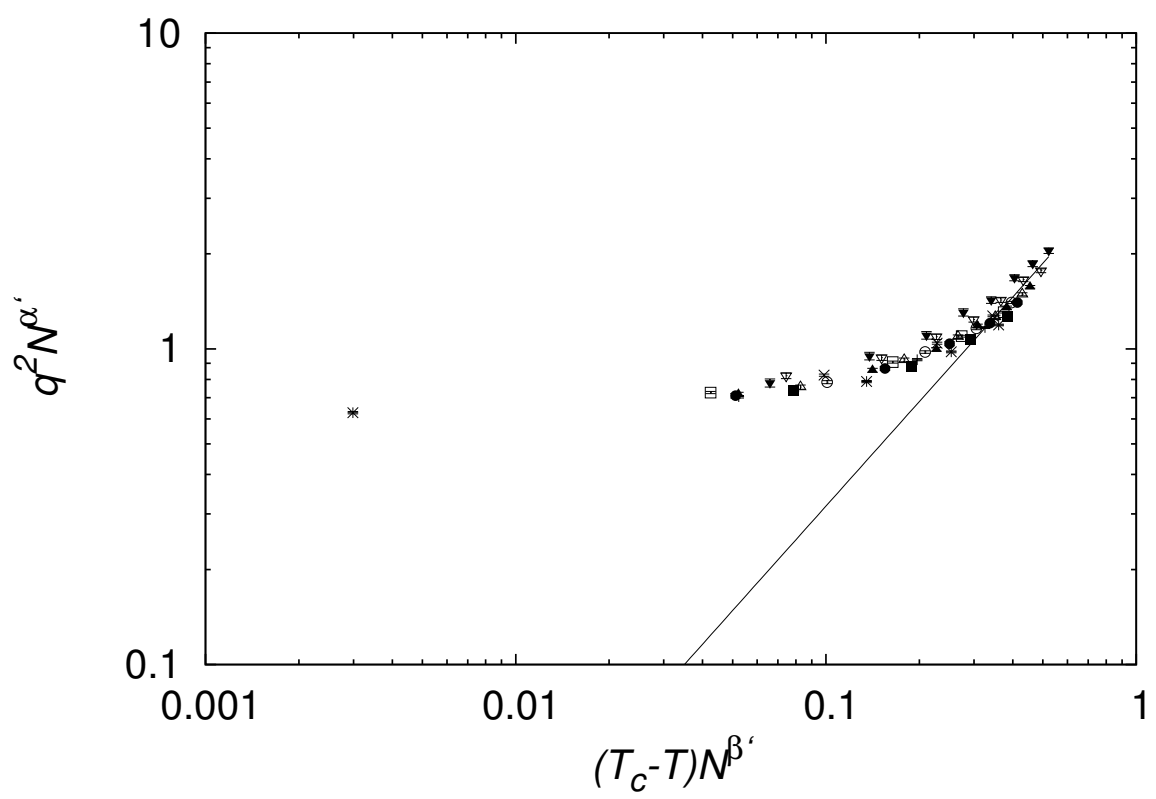

Figure 2.21: Double logarithmic scaling plot of $q^{2}$ with $k+1=8$ and $m=3$. Additionally plotted: $|x|^{1.1}$

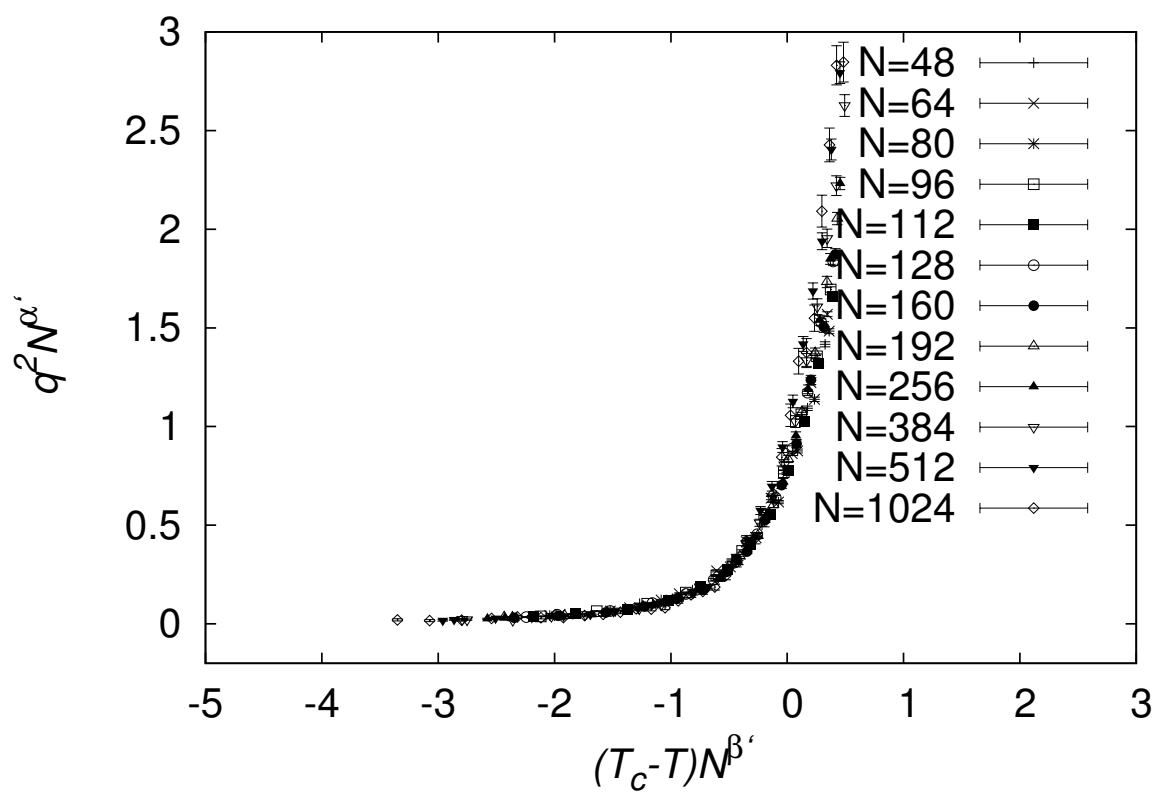

Figure 2.22: Scaling plot of $q^{2}$, with $k+1=8$ and $m=2$. 


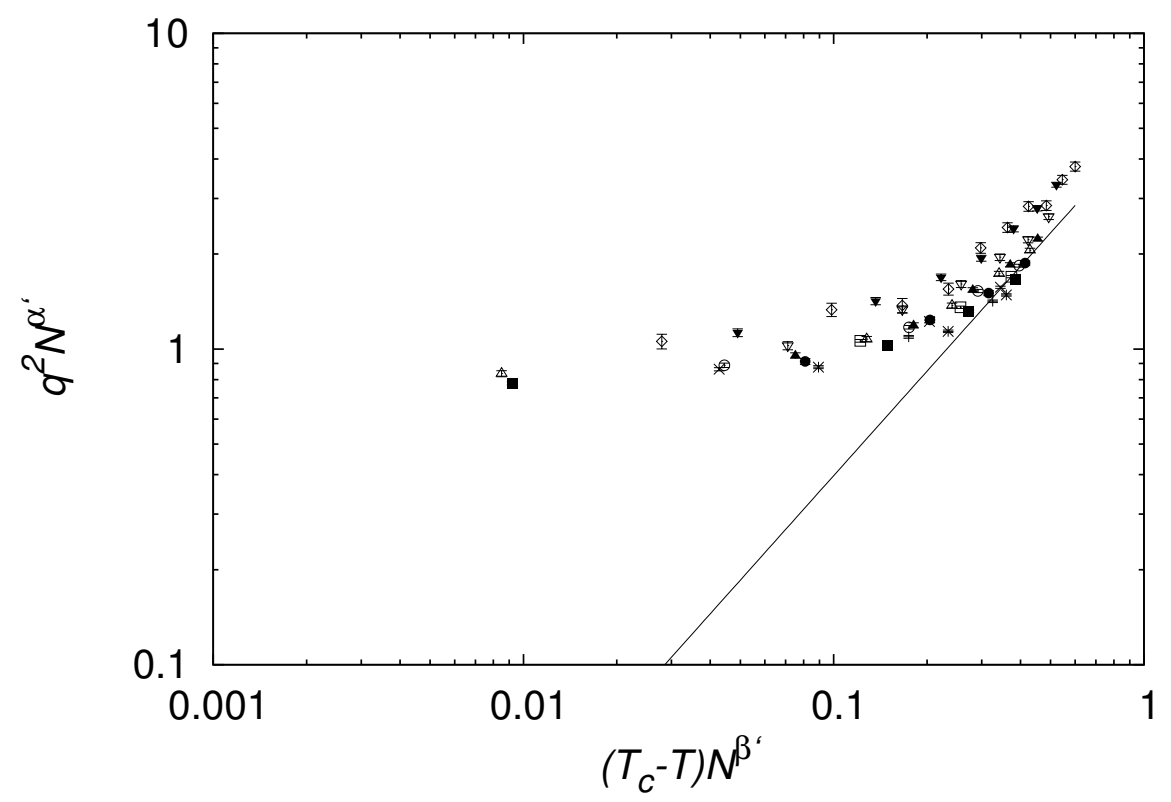

Figure 2.23: Double logarithmic scaling plot of $q^{2}$ with $k+1=8$ and $m=2$. Additionally plotted: $|x|^{1.1}$

Close to the critical temperature the self-consistent effective-field approach of the previous section is valid and we can define the free energy in terms of these fields. Generally, the free energy consists of site contributions $F_{i}^{(1)}$ of a single spin and bond contributions $F_{i j}^{(2)}$ of two neighbouring spins

$$
\begin{aligned}
F & =-k \sum_{i} F_{i}^{(1)}+\sum_{\langle i j\rangle} F_{i j}^{(2)} \\
& =-k \sum_{i}\left(-\frac{1}{\beta} \ln \operatorname{Tr} \rho_{i}^{(1)}\right)+\sum_{\langle i j\rangle}\left(-\frac{1}{\beta} \ln \operatorname{Tr} \rho_{i j}^{(2)}\right),
\end{aligned}
$$

with $\rho_{i}^{(1)}=\exp \left(\beta \sum_{j=1}^{k+1} \vec{\eta}_{i j} \vec{\sigma}_{i}\right)$ and $\rho_{i j}^{(2)}=\exp \left(\beta \sum_{l=1}^{k} \vec{\eta}_{i l} \vec{\sigma}_{i}+\beta \sum_{l=1}^{k} \vec{\eta}_{j l} \vec{\sigma}_{j}+\right.$ $\left.\beta J_{i j} \vec{\sigma}_{i} \vec{\sigma}_{j}\right)$, where we have split the sums defining the cavity field $\vec{h}_{i j}=\sum_{l \neq j}^{k} \vec{\eta}_{i l}$ and the local fields $\vec{H}_{i}=\sum_{l}^{k+1} \vec{\eta}_{i l}$ into contributions $\vec{\eta}_{i l}$ from each single neighbour $\vec{\sigma}_{l}$ (compare for instance to 2.7 ), where $\vec{\eta}_{i l}$ corresponds to $\frac{2 \beta J_{i l} \vec{h}_{i l}}{4 z_{i l}^{0}}$. Equation 2.32 can be derived starting from the usual definition $F=-\frac{1}{\beta} \ln e^{\beta \sum_{\langle i j\rangle} J_{i j} \vec{\sigma}_{i} \vec{\sigma}_{j}}$, explicitly not averaging over the disorder. Thereby the partition function of the single link between spin $\vec{\sigma}_{i}$ and $\vec{\sigma}_{j}$ can be written in terms of the cavity fields $\vec{h}_{i}$ (the field onto $\vec{\sigma}_{i}$ not taking into account 


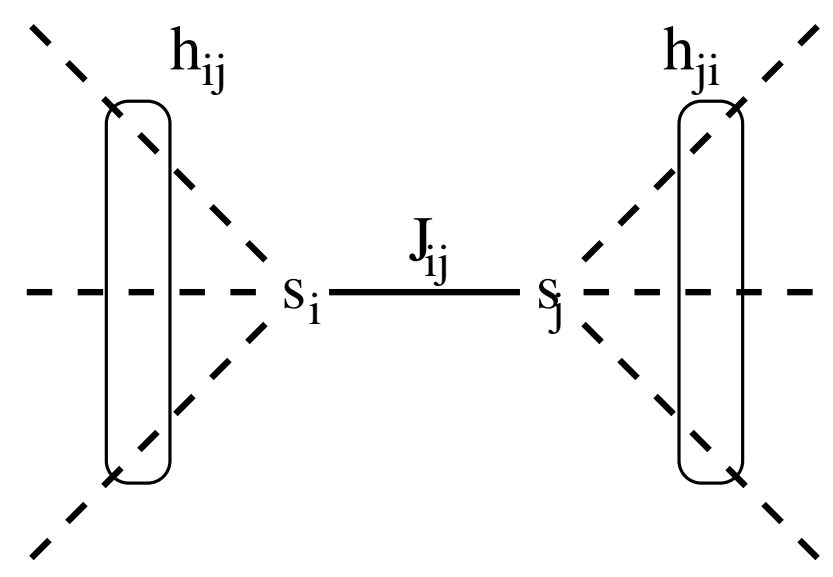

Figure 2.24: Two neighbouring spins $s_{i}$ and $s_{j}$ connected by $J_{i j}$ and the cavity fields onto them (indicated by the rectangular loops), not taking into account $J_{i j}$.

the link $J_{i j}$ ) and $\vec{h}_{j}$ (the field onto $\vec{\sigma}_{j}$ not taking into account the link $J_{i j}$ ), see figure 2.24. At first, we write the partition function as $\rho_{i j}^{(2)}$. However, summing over all links $J_{i j}$ of the lattice, we count the single contributions $\vec{\eta}_{i l}$ in $\vec{h}_{i j}=\sum_{l \neq j}^{k} \vec{\eta}_{i l}$ to every cavity field more than once, and we have to subtract redundant terms. In fact, the term $\vec{\eta}_{i l}$ appears in the sum of all but one of the $k+1$ cavity fields $\vec{h}_{i t}$ onto the spin $\vec{\sigma}_{i}$ (every single link $J_{i t}$ to one of the neighbours $\vec{\sigma}_{t}$ can be removed to describe one of these fields; If the link $J_{i l}$ is removed, the term $\vec{\eta}_{i l}$ does not contribute). Understanding further that the sum of all cavity fields onto a single spin $\vec{\sigma}_{i}, \sum_{l} \vec{h}_{i l}=\sum_{l} \sum_{j \neq l} \vec{\eta}_{i j}$, is $k$ times the true local field onto $\vec{\sigma}_{i}, \vec{H}_{i}$, the free energy resulting from $-\sum_{\langle i j\rangle} \frac{1}{\beta} \ln \operatorname{Tr} \rho_{i j}^{(2)}$ is

$$
\begin{aligned}
& -\frac{1}{\beta} \ln \operatorname{Tr} \exp \left(\beta \sum_{\langle i j\rangle} J_{i j} \vec{\sigma}_{i} \vec{\sigma}_{j}+\vec{h}_{i j} \vec{\sigma}_{i}+\vec{h}_{j i} \vec{\sigma}_{j}\right) \\
& \quad=-\frac{1}{\beta} \ln \operatorname{Tr} \exp \left(\beta \sum_{\langle i j\rangle} J_{i j} \vec{\sigma}_{i} \vec{\sigma}_{j}+\beta \sum_{i} \frac{k}{2} \vec{H}_{i} \vec{\sigma}_{i}+\beta \sum_{j} \frac{k}{2} \vec{H}_{j} \vec{\sigma}_{j}\right) .
\end{aligned}
$$

The factor $\frac{1}{2}$ in the last two contributions prevents to count each single link twice. As we find the usual expression for the free energy in the first part of equation (2.33), these additional contributions to the free energy have to be subtracted again. This is achieved by introducing $k$ times the site contribution $-k \sum_{i} F_{i}^{(1)}$ in equation (2.32). Therefore, we have to calculate $F_{i}^{(1)}=-\frac{1}{\beta} \ln \operatorname{Tr} \rho_{i}^{(1)}=-\frac{1}{\beta} \ln \operatorname{Tr} e^{\beta \vec{H}_{i} \vec{\sigma}_{i}}$ and $F_{i j}^{(2)}=-\frac{1}{\beta} \ln \operatorname{Tr} \rho_{i j}^{(2)}=$ 
$-\frac{1}{\beta} \ln \operatorname{Tr} e^{\beta J_{i j} \vec{\sigma}_{i} \vec{\sigma}_{j}+\beta \vec{h}_{i} \vec{\sigma}_{i}+\beta \vec{h}_{j} \vec{\sigma}_{j}}$. The first expression is

$$
\begin{aligned}
F_{i}^{(1)} & =\int d \sigma^{m} \delta\left(m-\vec{\sigma}^{2}\right) e^{\beta \vec{H}_{i} \vec{\sigma}_{i}} \\
& =\int d z_{i} e^{m z_{i}-\frac{1}{2} \ln \operatorname{det} M+\frac{\beta^{2}}{4} \vec{H}_{i}^{T} M^{-1} \vec{H}_{i}},
\end{aligned}
$$

where $M$ is a $m \times m$ matrix with only diagonal entries equal to $z_{i}$. The saddle point equations are $1-\frac{1}{2} z_{i}^{-1}-\frac{\beta^{2}}{4}\left(\frac{\vec{H}_{i}}{\sqrt{m}}\right)^{2} z_{i}^{-2}=0$. We solve them perturbatively with the ansatz $z_{i}=z_{i}^{0}+\Delta z_{i}$ and find $z_{i}^{0}=\frac{1}{2}$ and $\Delta z_{i}=\frac{\beta^{2} \vec{H}_{i}^{2}}{2 m}$. A site contribution then is

$$
-\beta F_{i}^{(1)}=m\left(\frac{1}{2}-\frac{1}{2} \ln \frac{1}{2}+\frac{\beta^{2} \vec{H}_{i}^{2}}{4 m z_{i}^{0}}\right) .
$$

The bond contribution is calculated from (substituting $\vec{h}_{i} \rightarrow \vec{h}_{i j}$ and $\left.\vec{h}_{j} \rightarrow \vec{h}_{j i}\right)$

$$
\begin{aligned}
F_{i j}^{(2)}=\int d \sigma_{i}^{m} d \sigma_{j}^{m} \delta\left(m-\vec{\sigma}_{i}^{2}\right) \delta\left(m-\vec{\sigma}_{j}^{2}\right) e^{\beta J_{i j} \vec{\sigma}_{i} \vec{\sigma}_{j}+\beta \vec{h}_{i j} \vec{\sigma}_{i}+\beta \vec{h}_{j i} \vec{\sigma}_{j}} \\
=\int d z_{i} d z_{j} e^{m\left(z_{i}+z_{j}\right)-\frac{1}{2} \ln \operatorname{det} A+\frac{\beta^{2}}{2} \vec{h}^{T} A^{-1} \vec{h}}
\end{aligned}
$$

where the $(2 m) \times(2 m)$ matrix $A$ has the entries $2 z_{i}$ and $2 z_{j}$ on the diagonal of the two diagonal $m \times m$ blocks resp., and $-\beta J_{i j}$ on the diagonal of the two $m \times m$ non-diagonal blocks

$$
A=\left(\begin{array}{cccccc}
2 z_{i} & & & -\beta J_{i j} & & \\
& \ddots & & & \ddots & \\
& & 2 z_{i} & & & -\beta J_{i j} \\
-\beta J_{i j} & & & 2 z_{j} & & \\
& \ddots & & & \ddots & \\
& & -\beta J_{i j} & & & 2 z_{j}
\end{array}\right) .
$$

It has the determinant $\operatorname{det} A=\left(z_{i} z_{j}-\left(\frac{\beta J_{i j}}{2}\right)^{2}\right)^{m}$. The vector $\vec{h}$ is a list of size $2 m$ with the entries of $\vec{h}_{i j}$ followed by the entries of $\vec{h}_{j i}$. The saddle point equations for $z_{i}$ and $z_{j}$ are

$$
\begin{aligned}
& 0=1-\frac{1}{2} \frac{z_{j}}{z_{i} z_{j}-\left(\frac{\beta J_{i j}}{2}\right)^{2}}+\frac{\beta^{2}}{4 m} \frac{\left(\frac{\beta J_{i j}}{2} \vec{h}_{j i}+z_{j} \vec{h}_{i j}\right)^{2}}{\left(z_{i} z_{j}-\left(\frac{\beta J_{i j}}{2}\right)^{2}\right)^{2}} \\
& 0=1-\frac{1}{2} \frac{z_{i}}{z_{i} z_{j}-\left(\frac{\beta J_{i j}}{2}\right)^{2}}+\frac{\beta^{2}}{4 m} \frac{\left(\frac{\beta J_{i j}}{2} \vec{h}_{i j}+z_{i} \vec{h}_{j i}\right)^{2}}{\left(z_{i} z_{j}-\left(\frac{\beta J_{i j}}{2}\right)^{2}\right)^{2}} .
\end{aligned}
$$


Again we solve them perturbatively with the ansatz $z_{i}=z_{i}^{0}+\Delta z_{i}$ and $z_{j}=$ $z_{j}^{0}+\Delta z_{j}$. As the equations in zeroth order are same, $0=1-\frac{1}{2} \frac{z_{j}^{0}}{z_{i}^{0} z_{j}^{0}-\left(\frac{\beta J_{i j}}{2}\right)^{2}}$ and $0=1-\frac{1}{2} \frac{z_{i}^{0}}{z_{i}^{0} z_{j}^{0}-\left(\frac{\beta J_{i j}}{2}\right)^{2}}$, we find $z_{i}^{0}=z_{j}^{0}=z_{0}=\frac{1}{4}+\frac{1}{4} \sqrt{1+4 \beta^{2} J_{i j}^{2}}$. The reduced $(m=1)$ determinant of $A$ in zeroth order simplifies to $\operatorname{det} A=z_{0}^{2}-\left(\frac{\beta J_{i j}}{2}\right)^{2}=$ $\frac{z_{0}}{2}$.

In first order, the saddle point equations are

$$
\begin{aligned}
& 0=-\frac{\Delta z_{j}}{z_{0}}+2\left(\Delta z_{i}+\Delta z_{j}\right)+\frac{\beta^{2}}{m} \frac{\left(\frac{\beta J_{i j}}{2} \vec{h}_{j i}+z_{0} \vec{h}_{i j}\right)^{2}}{z_{0}^{2}} \\
& 0=-\frac{\Delta z_{i}}{z_{0}}+2\left(\Delta z_{i}+\Delta z_{j}\right)+\frac{\beta^{2}}{m} \frac{\left(\frac{\beta J_{i j}}{2} \vec{h}_{i j}+z_{0} \vec{h}_{j i}\right)^{2}}{z_{0}^{2}} .
\end{aligned}
$$

We find the solution

$$
\begin{aligned}
& \left(4 z_{0}^{2}-z_{0}\right) \Delta z_{i}=-\frac{\beta^{2}}{m}\left(\vec{h}_{i j}^{2}\left(z_{0}^{2}+\left(\frac{\beta J_{i j}}{2}\right)^{2}\right)+\beta z_{0} J_{i j} \vec{h}_{j i} \vec{h}_{i j}\right) \\
& \left(4 z_{0}^{2}-z_{0}\right) \Delta z_{j}=-\frac{\beta^{2}}{m}\left(\vec{h}_{j i}^{2}\left(z_{0}^{2}+\left(\frac{\beta J_{i j}}{2}\right)^{2}\right)+\beta z_{0} J_{i j} \vec{h}_{i j} \vec{h}_{j i}\right) .
\end{aligned}
$$

With it, the bond contribution to the free energy up to first order is

$$
-\beta F_{i j}^{(2)}=m\left(2 z_{0}-\frac{1}{2} \ln \frac{z_{0}}{2}+\frac{\beta^{2}}{m} \frac{\left(\left(\vec{h}_{i j}\right)^{2}+\left(\vec{h}_{j i}\right)^{2}\right) z_{0}+\beta J_{i j} \vec{h}_{i j} \vec{h}_{j i}}{z_{0}}\right) .
$$




\section{Chapter 3}

\section{Generalized Bose-Einstein condensation}

At $T=0$, the $m$-component spin glass of finite size exhibits a characteristic property: For large component numbers $m$, the spins condense into a subspace of the original $m$-dimensional space they live in [45, 46]. They do not use all of the $m$ available components. Even more, the dimension of this subspace, $m_{0}$, scales with the system size, $m_{0} \sim N^{\mu_{m}}$, with a model dependent exponent $\mu_{m}$ [46, 47, 48, which is equal to $\frac{2}{5}$ for the fully connected SK-model and decreases for finite connectivities, see table 3.2. We call this phenomenon the generalized Bose-Einstein phase transition of vector spin glasses, as there is a sudden change of the behaviour of a finite system, when adding components to a $m_{0}$-dimensional spin glass and as this condensation generalizes the conventional Bose-Einstein condensation. The natural guess is that the spins use additional components to lower their free energy, but above $m_{0}$ they do not, but stick to the value of $m_{0}$. The variety of vector spin glass models that display this phenomenon indicates that it is a general feature of vector spin glasses in the large- $m$ limit. Due to the scaling property $m_{0} \sim N^{\mu_{m}}$, it can only be detected for finite $N$ (and $m>m_{0}$ ). As the explicit calculation of subextensive contributions in the partition function of spin glasses is impossible (due to the massless modes discussed in the introduction), we are restricted to investigate the phenomenon numerically. The question of interest in this chapter is whether a similar behaviour as in the ground state can be observed in the whole low temperature phase.

At $T=0$ there are no fluctuations, such that the regarding subspace the spins span can be directly observed, once a ground state configuration of spins is found. The investigation at finite $T$ is complicated by the fact that thermal fluctuations prevent to observe the subspace by solely regarding the spins $\vec{s}_{i}$. They preferably point into the direction of this subspace, but 
nevertheless fluctuate into the remaining directions as well. Fortunately, the local fields $\vec{H}_{i}$ do not fluctuate. These are the thermally averaged mean fields onto each spin $\vec{s}_{i}$ and we already know how to obtain them, since this was one of the concerns of chapter 2. The analysis of the dimension of the space the local fields span reveals the preferred direction the spins point into. This dimension displays a remarkable dependence on the temperature. To the best of our knowledge, this subspace has never been observed yet, neither numerically nor analytically. However, we now have a method at hand to examine this space.

We proceed as follows. In the next section, we present the methods and results of earlier investigations of this phenomenon for the $m$ component spin glass at $T=0$ [67]. In addition to the scaling exponent $\mu_{m}$, it is possible to obtain further scaling exponents and to find a scaling relation of the ground state energy in the large- $m$ limit [48], which will be of further use in this thesis. Afterwards, we present the observed behaviour for finite temperatures by using the numerical simulation of the self-consistent effective-field approach, presented in section 2.3, and by analysing the dimension of the space the local fields span. As the settling down of the local fields into a subspace of the originally $m$-dimensional space they live in is an ordering process as well, it gives helpful support to understand the phase transition discussed in the previous chapter.

\subsection{Effective Component Number at $T=0$}

Since there are no thermal fluctuations at zero temperature, the spins are aligned to the direction of their local fields $\vec{H}_{i}$. In general, a local field is the thermal mean of the sum of fields $J_{i j} \vec{s}_{j}$ produced by the neighbouring spins of $\vec{s}_{i}, \vec{s}_{j}$. As $\vec{s}_{i}$ aligns to its local field $\vec{H}_{i}$ and has the fixed length $\sqrt{m}$, we normalize the local field as $\frac{\vec{H}_{i}}{\sqrt{m}} \rightarrow \vec{H}_{i}$. Thus we have (with $H_{i}$ as the modulus of $\vec{H}_{i}$ )

$$
H_{i} \vec{s}_{i}=\sum_{j=1}^{k+1} J_{i j} \vec{s}_{j} .
$$

This equation is only valid in the ground state. In case of replica symmetry (i.e. for large $m$ ) there is a unique stable solution. Equation 3.1 is the basis for investigating the generalized Bose-Einstein phase transition at $T=0$.

To this end, we transform equation (3.1) into the eigenvalue equation $\sum_{j=1}^{N}\left(H_{i} \delta_{i j}-J_{i j}\right) s_{j}^{\alpha}=0$ (for each single component $\alpha=1, \ldots, m$ ) with the eigenvalue 0 . The matrix entries $H_{i} \delta_{i j}-J_{i j}$ are denoted by $A_{i j}$, and $A$ 
Table 3.1: Results for $\mu_{m}$ for different connectivities $k+1$

\begin{tabular}{|c|c|}
\hline$k+1$ & $\mu_{m}$ \\
\hline \hline$\infty[46]$ & $2 / 5$ \\
\hline $8[48]$ & $0.377( \pm 0.003)$ \\
\hline $6[48]$ & $0.362( \pm 0.004)$ \\
\hline $4[48]$ & $0.352( \pm 0.005)$ \\
\hline$d=3[47]$ & 0.33 \\
\hline$d=2[47]$ & 0.29 \\
\hline
\end{tabular}

is the inverse susceptibility matrix $\left(\chi^{-1}\right)$, see Ref. [46]. As the spins have $m$ components $\alpha$, there are $m$ of such eigenvalue equations. However, in Ref. 45] it was shown, that there can at most be $\sqrt{2 N}$ linearly independent eigenvectors to the eigenvalue zero. Their number determines the dimension of the null eigenspace of $A$. Further, the dimension of the null eigenspace is equal to the dimension of the space the spins live in. This can be seen by considering a matrix with the spins $s_{j}^{\alpha}$ as its entries. With $\alpha$ as the column index, the rows correspond to the spins itself and the columns of this matrix correspond to the null eigenvectors of $A$ (of which there are at most $m_{0}<\sqrt{2 N}$ linearly independent ones). As the column rank is equal to the row rank (and there are no thermal fluctuations at $T=0$ ), the spins span a $m_{0}$-dimensional subspace of the $m$-dimensional space they originally live in. We call the necessary number of components to observe this subspace the effective number of components and denote it by $m_{\mathrm{eff}}(T=0)=m_{0}$.

To find this subspace, we have to solve equation (3.1) to find groundstate configurations of the local fields. This is done by iterating this equation, where one calculates the local fields according to $\vec{H}_{i}=\frac{1}{\sqrt{m}} \sum_{j=1}^{k+1} J_{i j} \vec{s}_{j}$ (starting from some random configuration of spins) and aligning the spins to their local fields as $\vec{s}_{i}=\frac{\sqrt{m} \vec{H}_{i}}{H_{i}}$ afterwards. One stops repeating this procedure when the change of each of the local fields within one iteration step is sufficiently small. Then one can set up the matrix $A$ and diagonalise it numerically. Finally, the number of null eigenvalues of $A$ (which corresponds to the dimension of the null eigenspace) is equal to the dimension of the space spanned by the spins. In Ref. [46], the authors found $m_{0}$ in the fully connected model to scale with the system size as $m_{0} \sim N^{2 / 5}$. Additionally it was found that scaling of the form $m_{0} \sim N^{\mu_{m}}$ (with model dependent exponents $\mu_{m}$ ) holds for two- and threedimensional lattices [47] and Bethe lattices [67, 48], see table 3.1 . 


\subsection{EFFECTIVE COMPONENT NUMBER AT $T=0$}
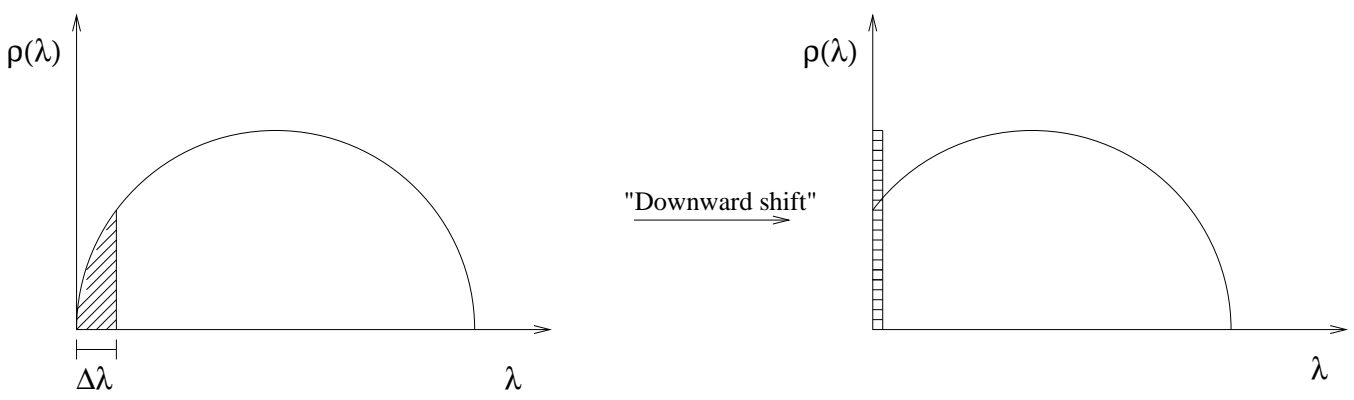

Figure 3.1: Downward shift of the semicircular eigenvalue density of the fully connected $m$-component spin glass: $m_{0}$ of the smallest eigenvalues (left, shaded) become null eigenvalues (deltapeak, right)

Is there an explanation for the observed values? In Ref. [46] is was shown that $\mu_{m}=2 / 5$ can be deduced as the result of the competition of two contributions to the ground state energy $e(m, N)=E /(N m)$. The first one is the ground state energy per spin and component in the limit $N \rightarrow \infty$ with $m$ large but fixed. It was derived in Ref. [41, 69] that it scales as $e_{0}+m^{-x}+\cdots$, with $e_{0}=-1$ and $x=1$ for the fully connected $m$-component spin glass. We apply the general formulation to finitely connected models, with the effective component number $m_{0}$, as $e_{0}+m_{0}^{-x}$ with $x$ unknown. This contribution prefers to choose $m_{0}$ as large as possible, i.e. $m_{0}=\sqrt{2 N}$. The other contribution arises from forcing the $N$ spins into a $m_{0}$-dimensional subspace, which is a significant shift of the ground state energy from above, as for finite system sizes, $m_{0}$ is expected to be of order $N^{\mu_{m}}$. Therefore, these energy costs vanish in the thermodynamic limit, but are important, because we are considering the limit $m \rightarrow \infty$ first. The amount of this contribution can be estimated by shifting the eigenvalue density of the matrix $A$, as described in figure 3.1. The eigenvalue density $\rho(\lambda)$ was suggested to scale as $\lambda^{\nu}$ for small $\lambda$ in Ref. [70]. For the fully connected model it corresponds to the Wigner semicircle law with $\nu=\frac{1}{2}$ as displayed in figure 3.1. but has a different appearance for finitely connected models [67, 48]. Nevertheless, the $m_{0}$ smallest eigenvalues occupy the space from 0 to $\Delta \lambda\left(\frac{m_{0}}{N}\right)^{1 / \nu+1}[67]$ and have to be shifted to zero. The necessary amount of energy for that shift is of the same order, as the sum of eigenvalues of $A$ (the Trace of $A$ ) is equal to $\sum_{i} H_{i}=\frac{1}{m} \sum_{i j} J_{i j} \vec{s}_{i} \vec{s}_{j}$. In total, we have to minimize $e=e_{0}+c_{1} m_{0}^{-x}+c_{2}\left(\frac{m_{0}}{N}\right)^{1 / \nu+1}+\cdots$ and find

$$
\mu=\frac{1}{x(\nu+1)+1} \text {. }
$$

As a check, we can insert $x=1$ and $\nu=\frac{1}{2}$ for the fully connected model 
Table 3.2: Results for the exponents $\mu_{m}, \nu, x$ and $y$ and the ground state energy per spin and component for different connectivities $k+1$.

\begin{tabular}{|c|c|c|c|c|}
\hline$k+1$ & $\nu$ & $x$ & $y$ & $e_{0}$ \\
\hline \hline$\infty$ & $1 / 2$ & 1 & $2 / 5$ & -1 \\
\hline 8 & $0.234( \pm 0.0003)$ & $1.34( \pm 0.002)$ & $0.505( \pm 0.004)$ & $-0.83( \pm 0.0016)$ \\
\hline 6 & $0.217( \pm 0.0003)$ & $1.45( \pm 0.002)$ & $0.525( \pm 0.006)$ & $-0.80( \pm 0.0015)$ \\
\hline 4 & $0.168( \pm 0.0013)$ & $1.57( \pm 0.0013)$ & $0.553( \pm 0.008)$ & $-0.73( \pm 0.0018)$ \\
\hline \hline
\end{tabular}

and get $\mu=\frac{2}{5}$, consistent with Ref. [46]. For the $m$-component spin glass on a Bethe lattice, this relation was used to derive the exponent $x$ from the findings of $\mu_{m}$ and $\nu$, see table 3.2 . There, the scaling of the eigenvalue density for small eigenvalues as $\lambda^{\nu}$ was analyzed via the integrated eigenvalue density [67, 48].

It is further possible to derive another scaling relation for the $m$-component spin glass, this time for the ground state energy per spin and component in the large- $m$ limit, as $e(m \rightarrow \infty, N)-e_{0} \sim N^{-y}$. On the one hand, as described above, we expect $e(m, N)-e_{0}$ to scale as $m_{0}^{-x}$ in the thermodynamic limit (i.e. for $N \rightarrow \infty$ ). On the other hand, for finite system sizes, the number of components $m_{0}$ scales with $N^{\mu_{m}}$. Therefore, we conjecture a combined scaling ansatz of the form $e(m, N)-e_{0}=m^{-x} \mathcal{F}\left(m N^{-\mu_{m}}\right)$. For $N \rightarrow \infty$, it scales as $m^{-x}$ (provided $\mathcal{F}(0) \neq 0$ ) as claimed by the definition of $x$. In the opposite limit, the large- $m$ limit, $m$ is larger than $m_{0} \sim N^{\mu_{m}}$ and we expect the ground state energy to be independent of $m$. Though $m$ might still be finite in that limit, we have the same ground state energy as if we had taken the limit $m \rightarrow \infty$. This is the characteristic property of the large- $m$ limit. It requires $\mathcal{F}(z)=$ const. $\cdot z^{x}$ for $m \geq m_{0}$, i.e. $z \geq m_{0} N^{-\mu_{m}}$, to cancel the prefactor $m^{-x}$ of the scaling ansatz. We denote this limit by $e(\infty, N)$ and find $e(\infty, N)-e_{0}=N^{-\mu_{m} x}$. The new scaling relation in the large- $m$ limit is

$$
y=\mu_{m} x=\frac{1-\mu_{m}}{1+\nu} .
$$

The second part of this equation follows from inserting the scaling relation (3.2). From figure 3.2 we see that this scaling law is fulfilled for $k+1=8$, within numerical precision. The ground state energy was calculated as $e(m, N)=-\frac{1}{2 N} \sum_{n} \lambda_{n}=\frac{1}{2 N m} \sum_{i j} J_{i j} \vec{s}_{i} \vec{s}_{j}$.

The scaling law holds for the other values of $k+1$ (data not shown). Unfortunately, the data are too noisy to deduce the exponent $y$ directly 


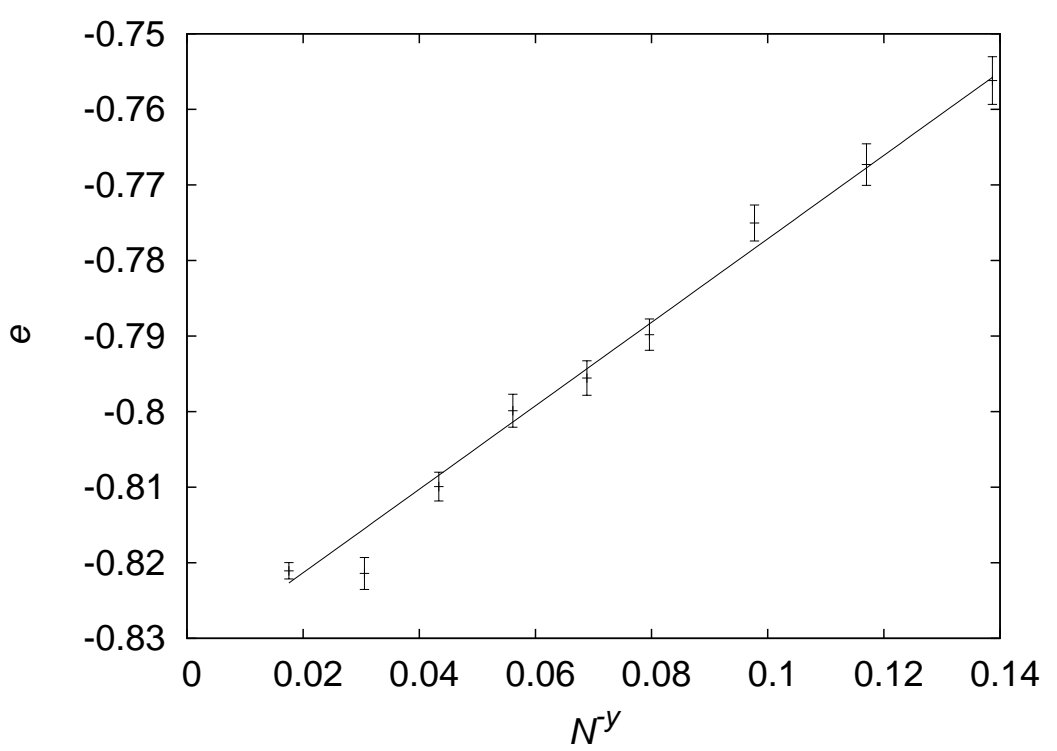

Figure 3.2: Ground state energy per spin and component for $k+1=8$ as a function of $N^{-y}$ with $y$ calculated from the scaling relation equation (3.3). The straight line extrapolates to the ground state energy $e_{0}$ in the limit $N \rightarrow \infty$.

from them, so the straight line in figure 3.2 has the slope $-y$, calculated from equation (3.3). For the fully connected model, equation (3.3) predicts $y=\mu_{m}=2 / 5$. Figure 3.3, which is a result of Ref. [46], shows that this is indeed observed.

In addition to the exponent $y$, the ground state energy $e_{0}$ after taking the limits $m \rightarrow \infty$ and $N \rightarrow \infty$ can be obtained by extrapolating the data as in figure 3.2 to $N=\infty$. The results are listed in table 3.2. As $k$ increases, $e_{0}$ converges towards -1 , the value for the fully connected model. While equation (3.2) is a result of Ref. [67], the derivation of the scaling relation (3.3) and the exponent $y$ is a result of this thesis and will be of further use in the last chapter.

\subsection{Effective Component Number for finite $T$}

In the last section we have learned that ground state spins of a $m$-component spin glass of finite size use only $m_{0}$ of their components in the large- $m$ limit. This effective number of components scales with the system size as $m_{0} \sim$ $N^{\mu_{m}}$. We now want to investigate the question whether this phenomenon occurs at finite temperature as well and if the same scaling relation holds. In 


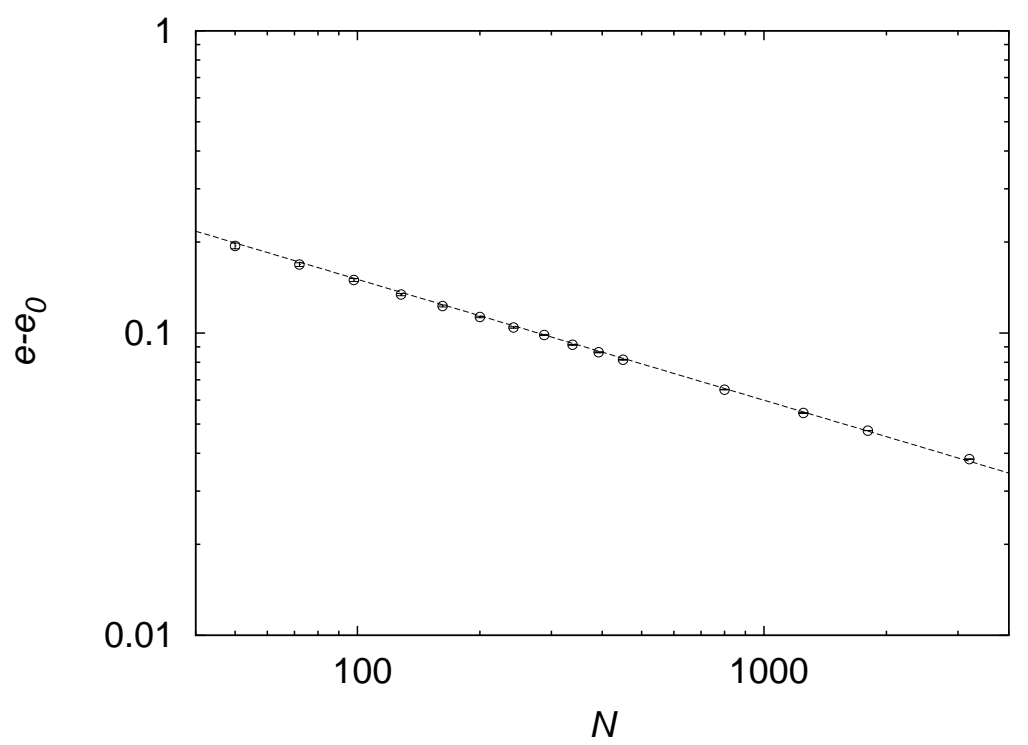

Figure 3.3: Ground state energy per spin and component for the fully connected model. The straight line has slope $-2 / 5$. The error bars are smaller than the point size.

general, the effective number of components in the low temperature phase, $m_{\text {eff }}(T)$, should be different than $m_{0}=m_{\text {eff }}(T=0)$. Nevertheless, the scaling relation $m_{\text {eff }}(T) \sim N^{\mu_{m}}$ should hold throughout the low temperature phase as we do not expect another phase transition.

As already pointed out in the introduction of this chapter, at finite temperature it is not the dimension of the space the spins span that we want to investigate. Due to thermal fluctuations they point in all of the available $m$ directions. However, they fluctuate around the direction of their local fields, $\vec{H}_{i}$, which are $m$ component vectors as well, but are not subject to thermal fluctuations as they are thermally averaged (or mean field) quantities, see chapter 2. If there is a phenomenon of a reduced component number at nonzero temperature, it should be displayed by the configuration of local fields. In chapter 2 we have learned how to obtain them: We iterate numerically the configuration of the effective cavity fields, $\vec{h}_{i j}$, for a given Bethe lattice until convergence (via equation (2.24)), and calculate the local fields according to equation (2.25) by inserting the effective fields $\vec{h}_{i j}$. To the best of our knowledge, this seems currently to be the only technique, how to obtain the local fields at nonzero $T$.

The investigation of the spin glass phase transition in the previous chapter was based on the assumption that iterating the configuration of effective fields 


\subsection{EFFECTIVE COMPONENT NUMBER FOR FINITE $T$}

$\vec{h}$ is the repeated application of the cavity equation $(2.7)$ (in the linearized small field approximation of equation (2.8) ) for each effective field, which we now summarize in the repeated application of a non-symmetric matrix $B$ onto the configuration of fields as $B \vec{h}$. Here $\vec{h}$ is a list of all $N(k+1)$ effective fields $\vec{h}_{i j}$. As each field $\vec{h}_{i j}$ has $m$ components, in fact it is a list of $N m(k+1)$ entries. Now we want to analyse the phase transition and the dimension $m_{\mathrm{eff}}(T)$ in terms of the matrix $B$.

The rows of $B$ correspond to the cavity equation for $\vec{h}_{i j}$, which is $\vec{h}_{i j}=$ $\sum_{l \in \mathrm{NN}(i) \neq j}^{k+1} \frac{2 \beta J_{i l} \vec{h}_{l i}}{4 z_{l i}^{00}}$, where the sum is over all cavity fields $\vec{h}_{l i}$ onto the $k+1$ neighbours $\vec{s}_{l}$ of $\vec{s}_{i}$ except neighbour $\vec{s}_{j}$. We may write the iteration of the configuration of effective cavity fields after convergence, $\vec{h}$, as an eigenvalue equation of the cavity matrix $B$

$$
\sum_{k l n^{\prime}} B_{i j n, k l n^{\prime}} h_{k l n^{\prime}}=\lambda h_{i j n}
$$

where $B$ has the entries $B_{i j n, k l n^{\prime}}=\frac{2 \beta J_{i k}}{4 z_{k i}^{00}} \delta_{n n^{\prime}} \delta_{j \in \mathrm{NN}(i)} \delta_{l \in \mathrm{NN}(k)} \delta_{i l}\left(1-\delta_{j k}\right)$, with $i, j, k, l=1, \cdots, N$ indicating the different cavity fields and $n, n^{\prime}=1, \cdots, m$ denoting the different components. As each spin has only $k+1$ neighbours, the matrix $B$ has $N m \times k$ nonzero entries. We see that it is not a symmetric matrix. However, as the entries are real, its characteristic polynomial has real coefficients. Therefore its roots, i.e. the eigenvalues $\lambda$ in equation (3.4), are either real numbers or conjugate pairs of complex numbers (if $a+i b$ is a root of the polynomial, than $a-i b$ is so, too). These eigenvalues of $B$ depend on the temperature. As long as their absolute values are smaller than one, the iteration will converge to the $\delta$-function around zero, i.e. to the paramagnetic solution (as the repeated application of the matrix $B$ in this case results in decreasing values of all fields until they have become zero). However, if we lower the temperature the eigenvalues increase and at some point the first eigenvalue has an absolute value equal to one, and we find $\vec{h}=B \vec{h}$ if this eigenvalue is real (after the configuration has ordered in the eigenbasis to this eigenvalue), i.e. a configuration with a stable non-vanishing variance. (In case of conjugate pairs of complex eigenvalues with an absolute value of one something different happens as will be discussed below.) If we lower the temperature further, the matrix $B$ is not appropriate to describe the true behaviour of the configuration of fields anymore, as it considers the small field approximation and the fields have already non-zero values. If we were able to calculate the eigenvalues of the true mapping of the cavity fields of an iteration step, $B_{\text {true }}$, (which includes solving the full cavity equation (2.7), involving nonlinear contributions), we expect that the largest eigenvalues stick to the absolute value of one, as stability of the configuration of fields 
does not allow for eigenvalues larger than one and we have found a stable variance of the configuration below $T_{c}$ in chapter 2, As the components of the configuration corresponding to the eigenvalues smaller than one do not survive the repeated application of the true matrix, it is the eigenspace of the eigenvalue one, which is the final basis of the configuration of effective fields. If there is a single (real) eigenvalue equal to one at the critical temperature, with all other eigenvalues smaller than one, the configuration of effective fields is one-dimensional.

We have been able to calculate the eigenvalue spectrum of $B$ and found that the distribution of eigenvalues is not homogeneous, as for some lattices the largest eigenvalue is a conjugate pair of complex values (i.e. $\lambda_{1,2}=a \pm i b$ ), whereas for other lattices it is indeed a single real eigenvalue. Even more, we find the eigenvalue spectrum of $B$ to be dominated by conjugate pairs of complex eigenvalues. So far, this phenomenon has not been reported (to the best of our knowledge). To find conjugate pairs of complex eigenvalues is a characteristic of rotation matrices. To see this, we regard, for instance, the two dimensional matrix $R$

$$
R=\left(\begin{array}{cc}
\cos \Theta & -\sin \Theta \\
\sin \Theta & \cos \Theta
\end{array}\right)
$$

which rotates objects in a two dimensional cartesian plane by an angle $\Theta$ around the origin. It has the eigenvalues $\lambda_{1,2}=\cos \Theta \pm i \sin \Theta$, i.e. a conjugate pair of complex values. From this we see that the configuration of effective fields is subject to rotations in the low temperature phase and that it is either one- or two-dimensional at the critical temperature. We illustrate the eigenvalue spectrum of $B$ for four different Bethe lattices of 64 spins and a connectivity of eight in Figure 3.4 (only the largest eigenvalues are plotted). From our findings we believe them to be representative for all system sizes and connectivities. Even more, we have analyzed the eigenvalue spectrum of the cavity matrix $B$ in case of Ising spins, i.e. with $\frac{2 \beta J_{i l} \vec{h}_{l i}}{4 z_{l i}^{0}}=u\left(J_{i l}, h_{l i}\right)$ [20], see section 2.1.1. The general picture of dominating conjugate pairs of complex eigenvalues holds in that case, too. Since $B$ holds only above and at the critical temperature, it is of no further use below $T_{c}$ (for instance to find the temperature at which the first real eigenvalue becomes equal to one, which might be a plausible indicator of a spin glass phase transition with freezing in time below $T_{c}$ ). Nevertheless, we will confirm the observations from above in the following. However, we should be aware of the fact that a rotating configuration is not frozen in time, though it can be described as stable in the sense of rotating all fields by the same angle. As a spin glass phase transition is, in general, expected to have a frozen configuration below $T_{c}$, our current results have profound consequences for the phase transition in 

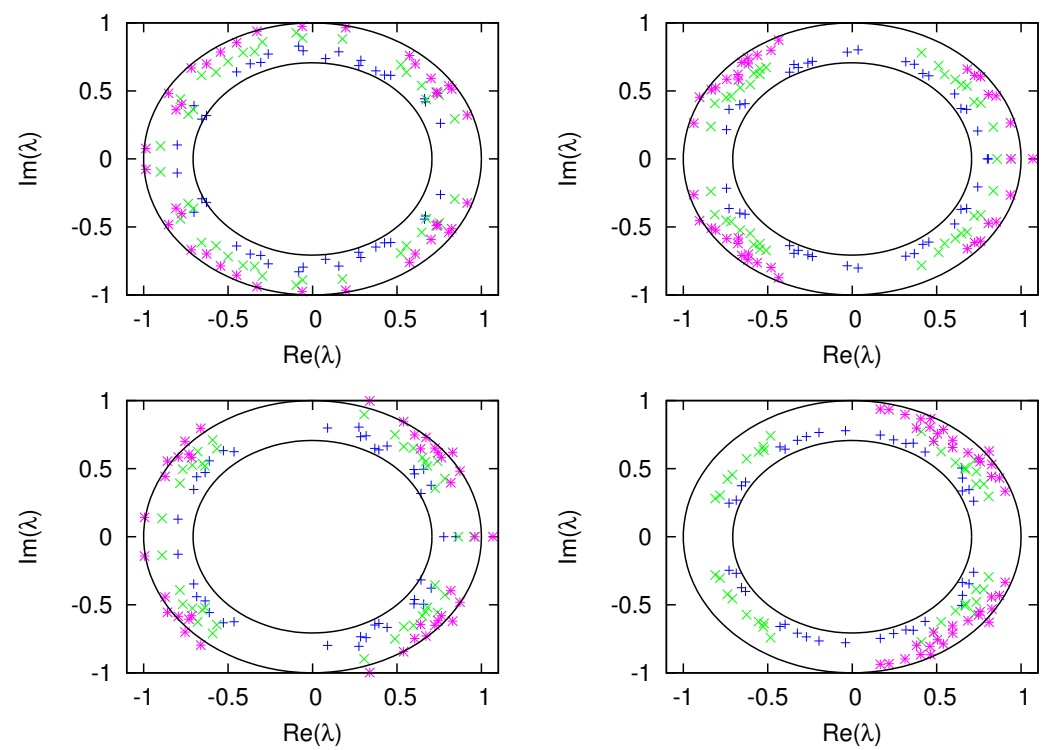

Figure 3.4: Eigenvalue spectra for four different Bethe lattices of 64 spins with $k+1=8$. Different colours represent different temperatures, decreasing from blue to green to pink. The larger circle has a radius of one. When the first eigenvalue crosses this circle, the phase transition happens. We see that some lattices have large real eigenvalues (lower left and upper right) and some have solely complex eigenvalues (lower right and upper left). 
chapter 2, in particular that it is not a spin glass phase transition of the usual type (though the spin glass phase transition is strongly supported by parallel tempering Monte Carlo simulations in section 2.4). We will compare the observations of both numerical methods at the end of this chapter and develop a common picture of the phase transition.

Now we want to investigate the space of local fields, for which we should observe the rotations due to conjugate pairs of complex eigenvalues of $B$ and the dimension of the space they span. Having found the local fields $\vec{H}_{i}$, what is this dimension? In the previous section we found $m_{0}$ at $T=0$ by diagonalising the quadratic, symmetric matrix $A$. This time, we have to diagonalise a $N \times m$ non-symmetric matrix $M$, with the entries $H_{i l}, i=1, \ldots, N$ and $l=1, \ldots, m$. The method of choice is a singular value decomposition $M=U D V^{T}$ [71], in which a numerical routine calculates biorthogonal leftand right-eigenvectors of the matrix $M$, which are the building columns of the unitary matrices $V$, resp. $U$. The singular values are the entries of the diagonal $m \times m$ matrix $D$. The rank of $M$, which is the dimension $m_{\text {eff }}(T)$ of the space the local fields span, is equal to the number of non-zero singular values in $D$ (the non-zero singular values we find are of order 1 whereas all other singular values are at least five orders of magnitude smaller and are not expected to contribute to the rank of $M$ ). We find a remarkable dependence of $m_{\mathrm{eff}}(T)$ on the temperature. Above $T_{c}$ all fields are zero, so the effective dimension of the space of local fields is zero. However, close to the critical temperature we find an increase of the number of effective components with either a value of 1 or 2 at $T_{c}$ (which is the temperature where the variance becomes non-zero). Below the critical temperature, $m_{\text {eff }}(T)$ increases slowly until the value of $m$ is reached. This is illustrated in the plots 3.5-3.8 for various Bethe lattices, system sizes and connectivities. Additionally to $m_{\mathrm{eff}}(T)$, the variance of the configuration of effective fields (i.e. the order parameter) is plotted, which indicates the phase transition at that temperature where the variance becomes non-zero, i.e. these plots are a direct continuation of the results from section 2.3. The critical temperature from this indication fits very well with the temperature at which $m_{\text {eff }}(T)$ is smallest. We further see that the effective number of components $m_{\mathrm{eff}}(T)$ increases with a step size of two instead of one in most cases below $T_{c}$. This is a consequence of the dominating influence of conjugate pairs of complex eigenvalues with nonvanishing imaginary parts. Remarkably, even for the rather low maximum component number of $m=4$ in figure 3.8 not all components are used at the critical temperature and we are in the large- $m$ limit (at $T_{c}$ ). We expect the increasing of $m_{\mathrm{eff}}(T)$ above $T_{c}$ to be a numerical effect, i.e. the largest eigenvalues, though still smaller than one, already dominate the configuration of fields. In fact, the transition is indicated by the instability of the $\delta$-solution 


\subsection{EFFECTIVE COMPONENT NUMBER FOR FINITE $T$}

of the distribution of effective fields (where all fields are zero), see chapter 2 .
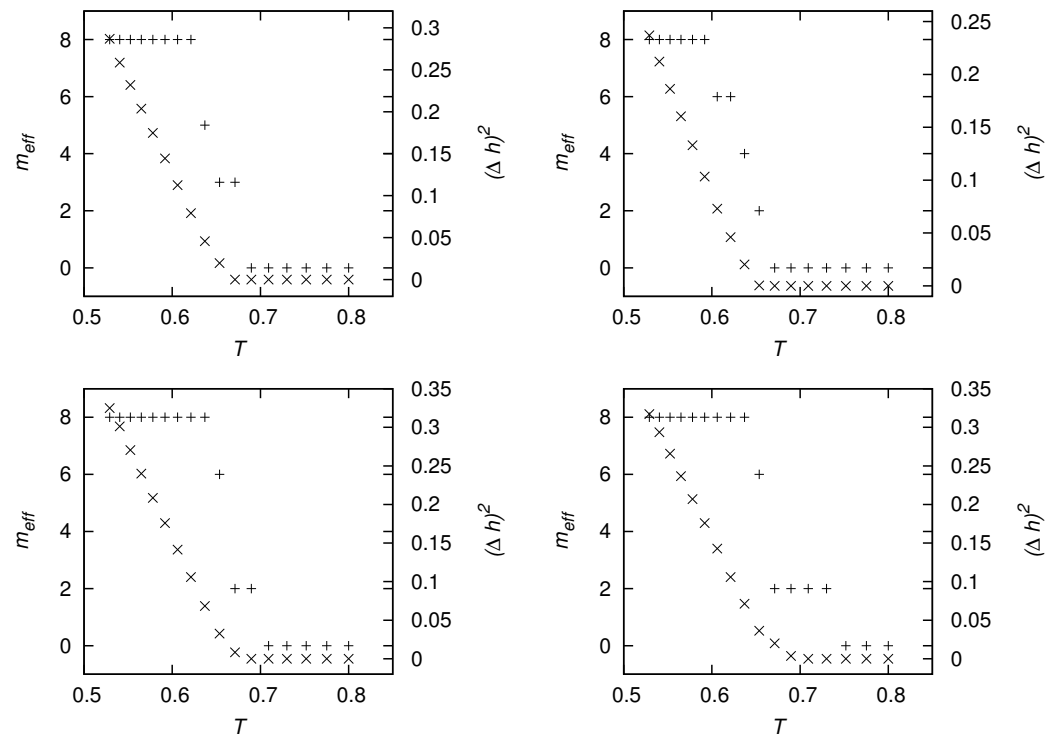

Figure 3.5: Effective number of components $m_{\mathrm{eff}}(T)(+)$ for 256 spins with 8 components on different Bethe lattices with $k+1=8$. Additionally plotted is the order parameter from section $2.3(\times)$.

The observation of $m_{\mathrm{eff}}\left(T_{c}\right)=2$ confirms what we have already derived by regarding the eigenvalues of the non-symmetric matrix $B$. At the critical temperature the configuration of local fields is not frozen in time in that case but it is two-dimensional and rotates in this two-dimensional plane (according to the largest eigenvalue which is a conjugate pair of complex values), see figures 3.9. 3.10 and 3.13. There, the order parameter (which is the variance of the configuration) remains unchanged and each single field keeps its length. A detailed look at the rotation of the local fields reveals that it is not only the whole configuration which is rotated, but every single field $\vec{H}_{i}$ rotates about the same angle, see figures 3.11 and 3.13. To see this, we calculated the angle between the old fields and the new fields onto each spin $\vec{s}_{i}$. The new field is found by calculating a new configuration of effective fields via $\vec{h}_{\text {new }}=B \vec{h}_{\text {old }}$. The new effective fields determine the new local fields. For all temperatures with $m_{\mathrm{eff}}(T)=2$ we find the same angle between each of the old fields and its corresponding new field, i.e. the relation between each pair of spins remains the same under these rotations. We see that at the phase transition (below which we initially thought to be freezing in time) in that case exists a stable configuration up to the global rotation of all local fields and that these rotations cost no energy, as the angles between each two of 

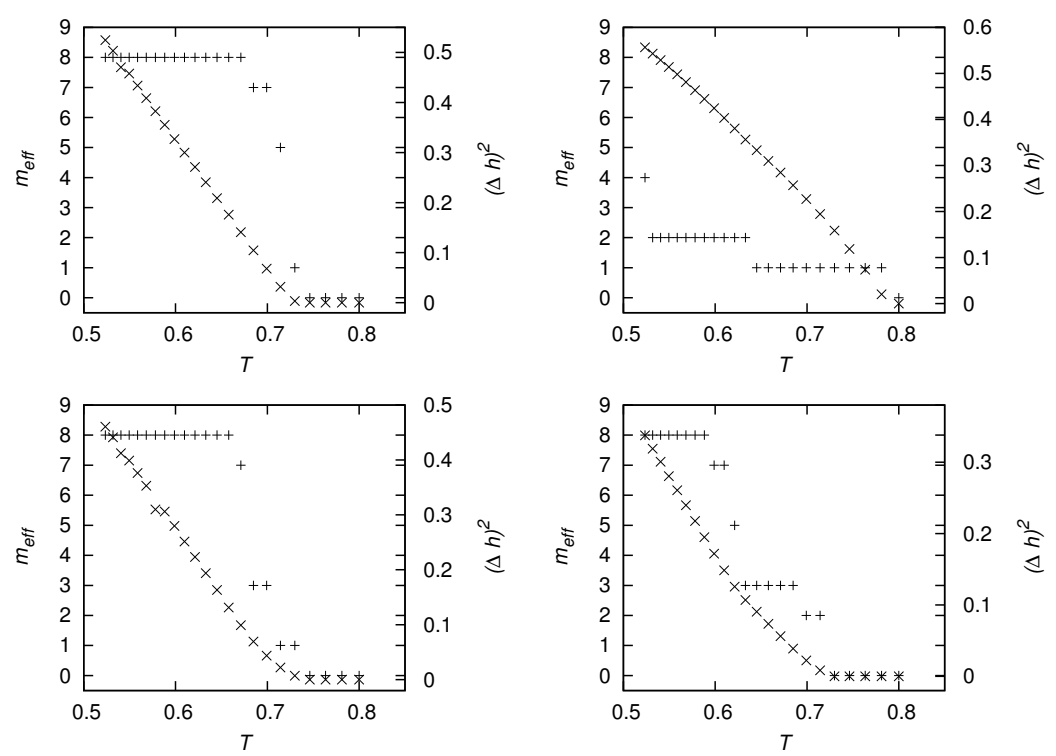

Figure 3.6: Effective number of components $m_{\mathrm{eff}}(T)(+)$ and the order parameter from section $2.3(\times)$ for 96 spins with 8 components on different Bethe lattices with $k+1=8$.
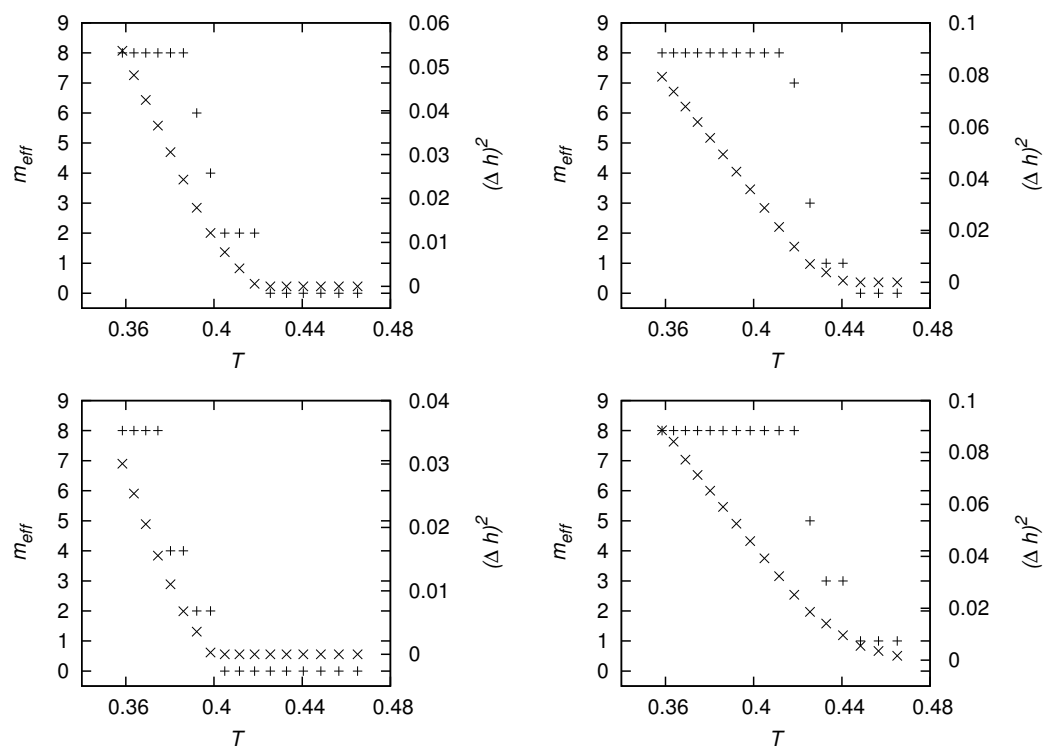

Figure 3.7: Effective number of components $m_{\mathrm{eff}}(T)(+)$ and the order parameter from section $2.3(\times)$ for 256 spins with 8 components on different Bethe lattices with $k+1=4$. 


\subsection{EFFECTIVE COMPONENT NUMBER FOR FINITE $T$}
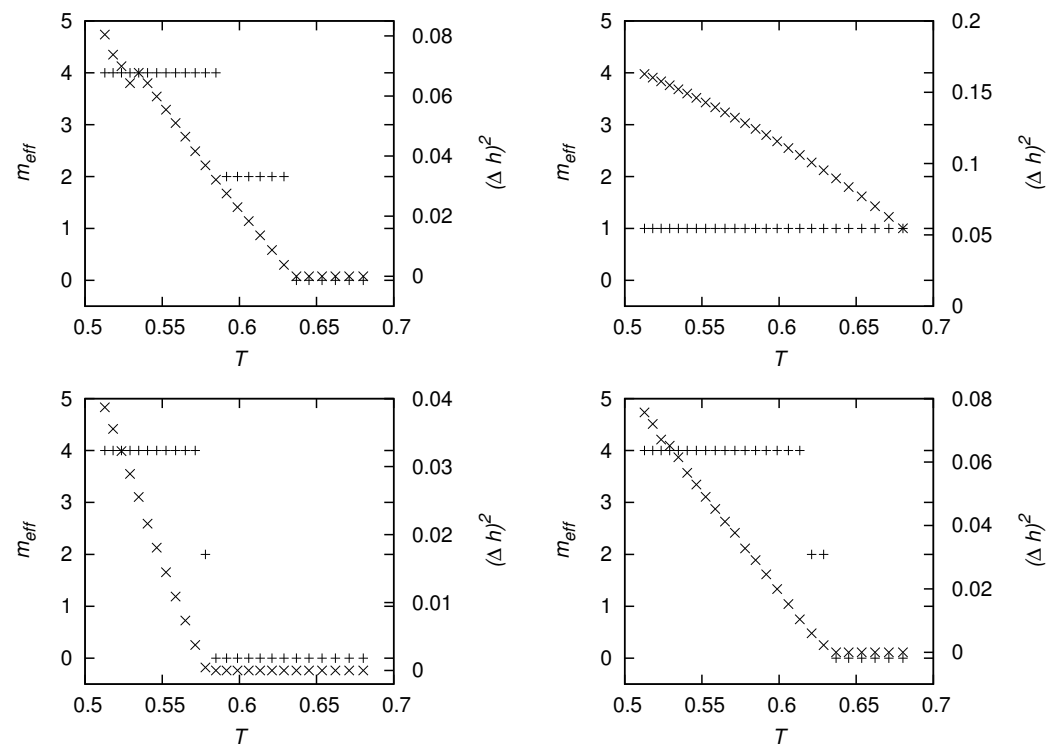

Figure 3.8: Effective number of components $m_{\mathrm{eff}}(T)(+)$ and the order parameter from section $2.3(\times)$ for 512 spins with 4 components on different Bethe lattices with $k+1=6$.

the fields are constant. Below $T_{c}$ more and more of these rotations occur due to the increasing number of conjugate pairs of complex eigenvalues of the full version of the cavity matrix, $B_{\text {true }}$, which are supposed to stick at an absolute value of one, resulting in a complex change of the configuration of fields in each single iteration step. In that case each effective cavity field is represented by a different eigenvalue decomposition. Therefore, in each iteration step the different fields are subject to different rotations (with possibly different axis and angles of rotation). Hence, the fields do not rotate parallel anymore. In case of $m_{\mathrm{eff}}\left(T_{c}\right)=1$ there is freezing in time until, for lower temperatures, a rotation sets in when the second (or simultaneously the third) eigenvalue becomes equal to one. These rotations are not parallel for the local fields (data not shown) for the same reason as above. Our findings state that the continuous symmetry of vector spins exhibits surprising replica symmetric equilibrium states.

Below $T_{c}$ the effective number of components increases up to the maximal value $m$. From the analytic result of a broken replica symmetry for finite $m$ in the thermodynamic limit [41], we suppose that replica symmetry holds (at least) as long as $m_{\text {eff }}(T)<m$, which we call the large- $m$ limit. We will confirm this conjecture in the next chapter. However, if $m$ is large enough, we should be able to come close to $T=0$ and still have $m_{\mathrm{eff}}(T)<m$. In that 


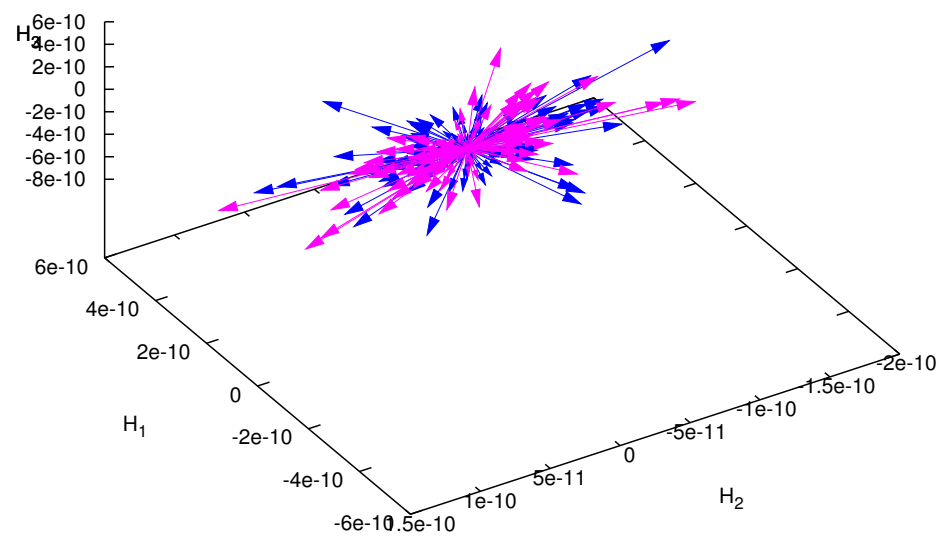

Figure 3.9: Three-dimensional plot of a configuration of local fields (128 spins with three components) in the low temperature phase before (blue) and after (pink) a single iteration step. Each configuration is only two-dimensional for this lattice at the chosen temperature, i.e. the large- $m$ limit holds.

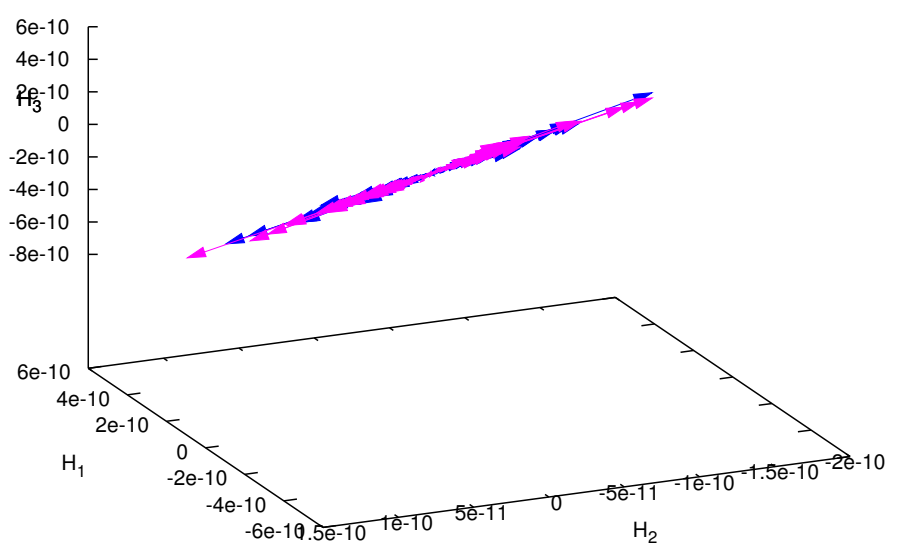

Figure 3.10: Different view at plot 3.9. The rotation of the iteration step takes place in the two-dimensional plane each configuration spans. 


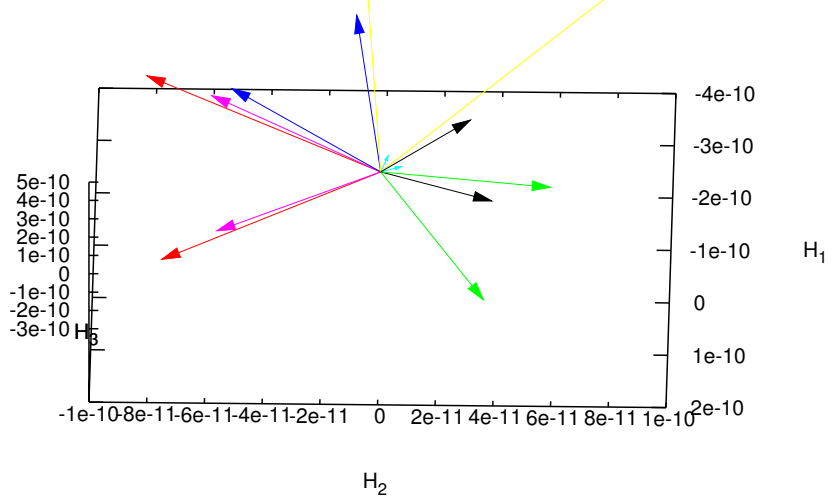

Figure 3.11: Seven local fields from figure 3.9 before and after a single iteration step. Each field is rotated by the same angle.

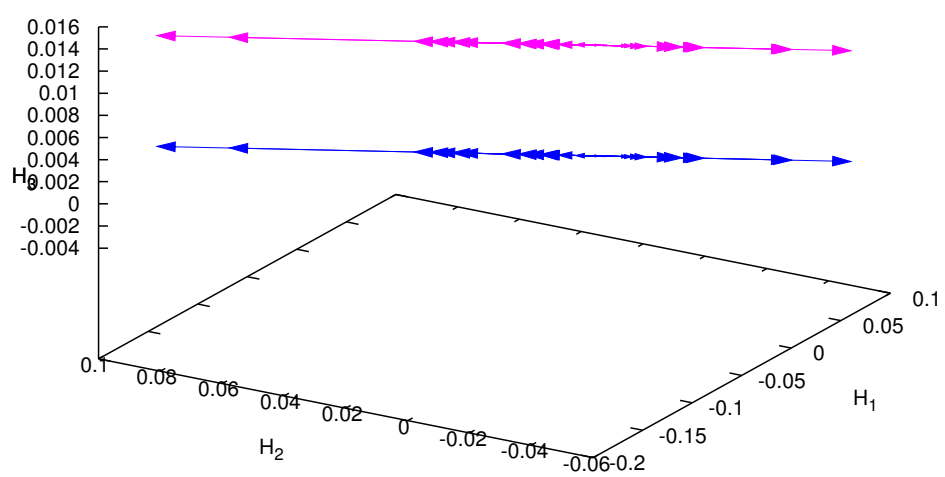

Figure 3.12: Three-dimensional plot of a configuration of local fields (128 spins with three components) in the low temperature phase before (blue) and after (pink) a single iteration step (the pink configuration is shifted slightly upwards $(+0.01)$ to distinguish it from the blue one). In contrast to figure 3.9 each configuration is one-dimensional and there is no rotation for this lattice at the chosen temperature. 

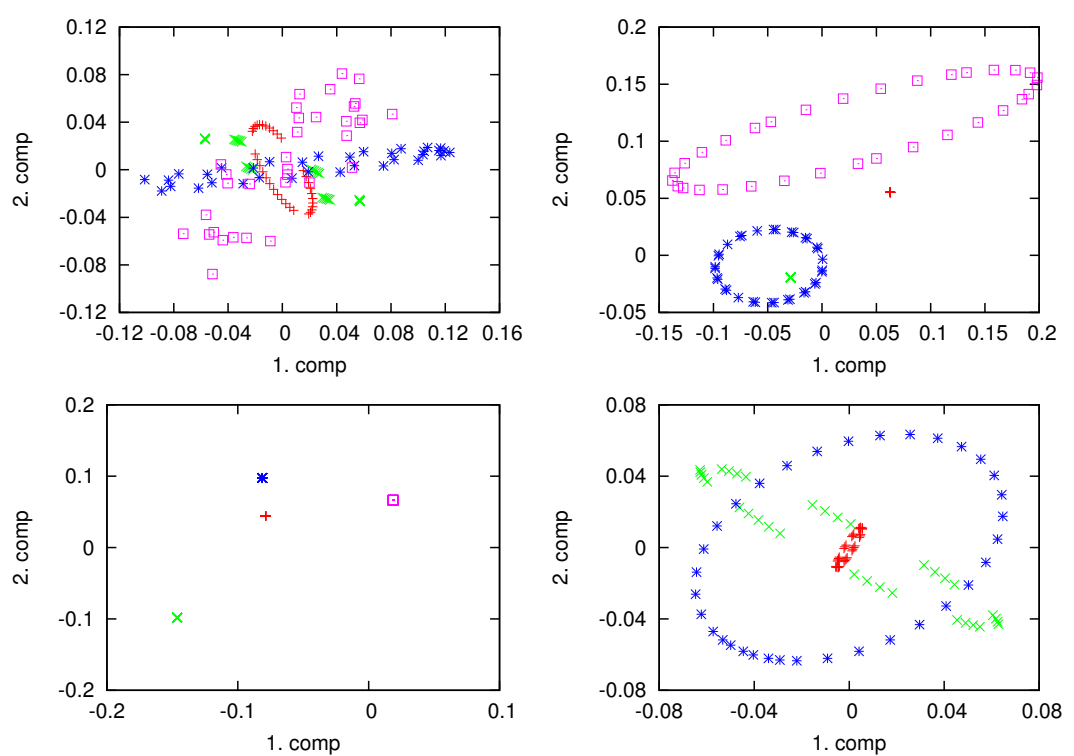

Figure 3.13: First and second component of a single effective cavity field for four different Bethe lattices of 64 spins with $k+1=8$. Different colours represent different temperatures, decreasing from red to green to blue to pink. The plot presents the different possible scenarios: The field is frozen in time (lower left plot and single red or green cross in upper right plot), rotations (ellipses, e.g. lower and upper right plot) or a mixture of rotations and fixed contributions, possibly rotating in a space the size of which is larger than the available $m$ components (pink boxes in upper left plot). 


\subsection{EFFECTIVE COMPONENT NUMBER FOR FINITE $T$}

way, we would find an estimate of $m_{0}$ from section 3.1. As we are using the truncated model, i.e. regarding solely the vector fields and neglecting higher orders, which is only valid close to the critical temperature, we expect the estimate of $m_{0}$ not to coincide with the value from section 3.1, for instance, the value of $m_{\mathrm{eff}}(0.6)=8$ from figure 3.5 is already larger than the value of $m_{0}$ from section 3.1 .

Can we relate these observations to those of the parallel tempering Monte Carlo method we used in section 2.4. As already stated, the critical temperatures from both methods coincide very well. This is strong support for a phase transition. The nature of this transition is, however, uncommon. In this section we have found that it is accompanied by an unusual phase transition, the generalized Bose-Einstein condensation, in which the spins, resp. the local fields, use only a subspace of the available $m$-dimensional space. Furthermore, the configuration of fields is subject to rotations in the low temperature phase. These rotations are reminiscent of Goldstone modes [53], which describe excitations of an equilibrium state in vector spin glasses without energy cost, due to the invariance under rotations in the spin space. However, Goldstone modes are thermal excitations, whereas the local fields are of mean field character and should not display thermal excitations. Therefore the observation of a rotating configuration of local fields is a phenomenon which we find to be in contrast to Goldstone modes. On the other hand, in case of the parallel tempering Monte Carlo method, we observe power-law scaling of the order parameter (which is related to the correlation function) with the system size. This is in fact a property which is reminiscent of a Kosterlitz-Thouless transition, where thermal fluctuations in directions transverse to the one the system wants to order destroy the phase transition (by exhibiting power law scaling of the correlation function with the system size, i.e. vanishing long range order in the thermodynamic limit). In our model, the order parameter can be rescaled by a factor $m$ to be finite in the low temperature phase, though the scaling property still holds (but with an exponent different from the theory of Kosterlitz-Thouless transitions). Unfortunately, within the parallel tempering Monte Carlo simulations we can not distinguish between Goldstone modes and a configuration of local fields rotating by itself. However, the strong support for a phase transition and the indicated relation to phenomena resulting from Goldstone modes makes it sensible to suspect the phase transition of the $m$-component spin on a Bethe lattice to be dynamically driven by rotations inherent in the phase transition, a phenomenon similar but different to Goldstone modes.

To further support the conjecture of the kind of phase transition described above, we derive a finite size scaling ansatz for the effective number of components. It regards the apparently universal scaling with $1 \leq m_{\mathrm{eff}}\left(T_{c}\right) \leq 2$ 
at the critical temperature and the scaling with the system size as $m_{\text {eff }}(T) \sim$ $N^{\mu_{m}}$, with $\mu_{m}$ from section 3.1 (We expect this scaling with the system size to hold in the whole low temperature phase, following the line of argument from section 3.1). We propose the ansatz

$$
m_{\mathrm{eff}}(T)=c+N^{\mu_{m}} \mathcal{F}\left(\left(T_{c}-T\right) N^{a}\right) .
$$

The scaling function $\mathcal{F}(x)$ has the properties of $\mathcal{F}(0)=0$ and $\mathcal{F}(x) \rightarrow$ const. for $|x| \rightarrow \infty$. The universal value of $c$ at $T_{c}$ is in between 1 and 2 . The figures 3.14 (bare data) and 3.15 (scaled data) show the resulting finite size scaling plots. The data match very well in the vicinity of the phase transition in figure 3.15 (i.e. close and right to the value of zero at the x-axis). The crossover at $T \approx 0.69$ for $m_{\mathrm{eff}}(T) \approx 2$ is clear to see in figure 3.14 . For $m_{\text {eff }}(T)=0$ the data are not supposed to match according to the scaling ansatz. When $m_{\text {eff }}(T)$ reaches its maximum value of $m=8$, the data splay out as well as expected. With $\mu_{m}=0.377$ and $T_{c}=0.69$ from the previous chapters, we find $c \approx 2, a \approx 0.6$ in the best matching scaling plot.

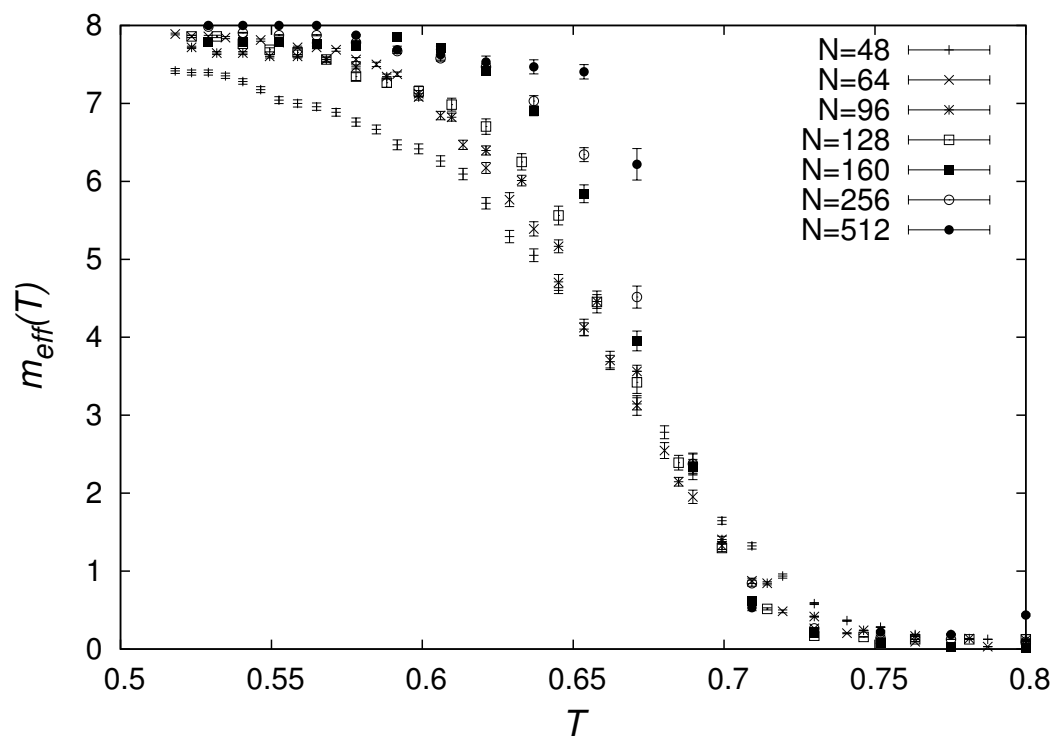

Figure 3.14: Unscaled effective component number $m_{\text {eff }}(T)$ for different system sizes.

The observation of $m_{\mathrm{eff}}\left(T_{c}\right)=2$ has so far, to the best of our knowledge, not been reported in the literature. Nevertheless, we expect this phenomenon to be a general property of vector spin glasses, as the results from the different methods we use complement each other to a sensible common picture. 


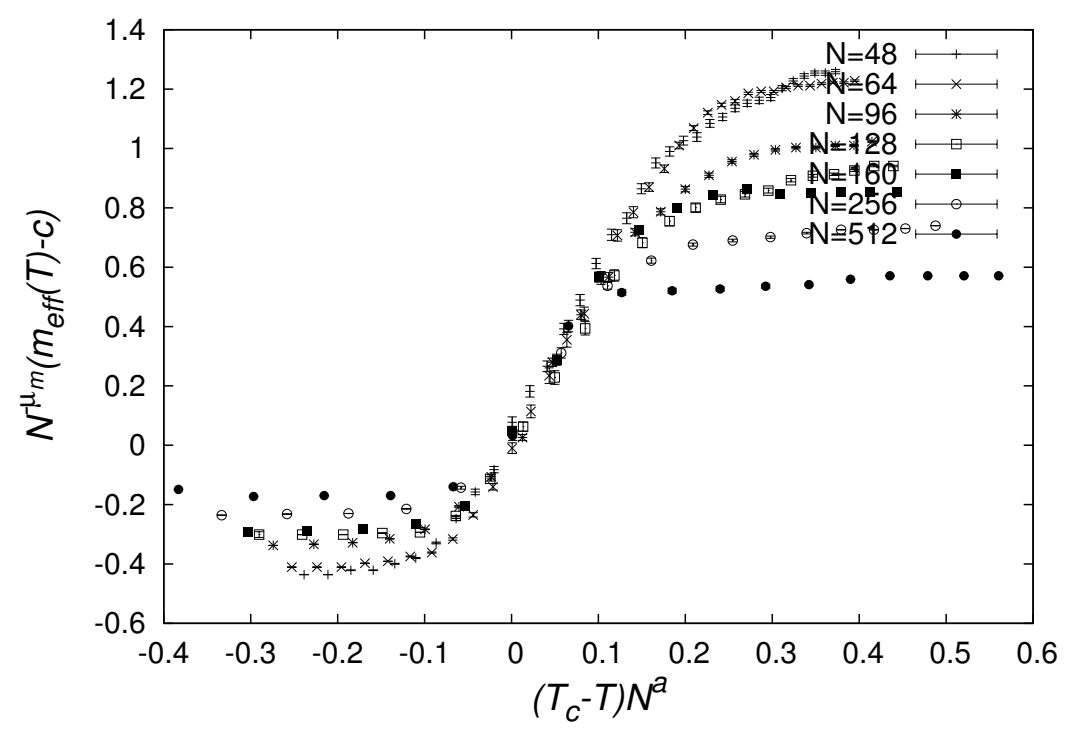

Figure 3.15: Scaling plot for $N^{-\mu_{m}}\left(m_{\mathrm{eff}}(T)-c\right)$ against the scaling function $\mathcal{F}\left(\left(T_{c}-T\right) N^{a}\right)$.

However, we are lacking of an intuitive explanation of it. In general, it seems sufficient to break the discrete symmetry of the Ising spins and introduce a continuous symmetry of the spins to find the large- $m$ limit at and, at least in a small temperature range, below the critical temperature. The results of this chapter will contribute to explain the stability and breaking of replica symmetry in the following chapter. 


\section{Chapter 4}

\section{Breaking Replica Symmetry}

Replica symmetry breaking is the ansatz with which the SK-Ising spin glass model can be solved. This has been a milestone in the history of spin glasses, as the first attempts of solving this fundamental solvable model of a spin glass with a replica symmetric ansatz in Ref. 2] displayed unphysical solutions in Ref. 6]. Later on, the technique to examine the stability of the replica symmetric solution was extended to the SK model of vector spin glasses. In Ref. [41] it was shown that replica symmetry of the fully connected $\mathrm{m}$ component spin glass is broken for all finite $m$. Replica symmetry only holds for $m \rightarrow \infty$. However, the calculation is based on a saddle point evaluation for $N \rightarrow \infty$, i.e. in the thermodynamic limit. We expect this discovery to be true for finite connectivities as well, but in the previous chapter we have seen that there is an effective number of components $m_{\mathrm{eff}}(T)$ to form a replica symmetric spin glass state for finite system sizes $N$. Above this value, i.e. for $m>m_{\mathrm{eff}}(T)$, the $m$-component spin glass is independent of $m$. As we expect $m_{\text {eff }}(T)$ to scale with the system size, as in equation (3.6), we suppose it is infinite in the thermodynamic limit, which would break the symmetry between replicas for all finite $m$ in that case. In contrast, for finite $N$, we combine these observations to the conjecture that replica symmetry holds as long as the spin glass has more components than necessary, i.e. $m \geq m_{\mathrm{eff}}(T)$. This is what we call the large-m limit and we are going to examine this conjecture in this chapter.

In the first section we present a simple technique how to distinguish replica symmetry and broken replica symmetry in the simulations of a configuration of effective cavity fields of section 2.3. We will see that replica symmetry is indeed broken when $m_{\text {eff }}(T)$ crosses the maximum number of components $m$. In section 4.2, we use the parallel tempering Monte Carlo algorithm to investigate the variance of the link overlap $q_{L}$ of two replicas of the $m$-component spin glass with the same configuration of disorder. The 
link overlap is a measure of correlation between the two copies and its variance can be used to distinguish between replica symmetry and broken replica symmetry, as will be explained there. In the last section of this chapter, we implement a calculation, in which we introduce two different corrections to the fully connected, large- $m$ spin glass. On the one hand these are corrections for finite connectivities and on the other hand for finite component numbers. The calculation involves adjusting a technique of Goldschmidt and de Dominicis [72], which was developed for Ising spins, to calculate the overlaps $q_{\alpha \beta}^{\mu \nu}=\left\langle\sigma_{\alpha}^{\mu} \sigma_{\beta}^{\nu}\right\rangle$ of two components $\mu$ and $\nu$ of the same spin $\vec{\sigma}$ taken from possibly different copies (replicas) $\alpha$ and $\beta$ of the same spin glass. Both copies can be in different equilibrium states, if replica symmetry is broken. That is why we can distinguish both scenarios. As in section 2.2, we present a procedure how to examine finite component numbers. By comparing the results for $q_{\alpha \beta}^{\mu \nu}$ of a replica symmetric ansatz and a one step broken replica symmetry ansatz, we try to find evidence for the breaking of replica symmetry for finite $m$ in the thermodynamic limit. Additionally, we are able to calculate corrections for finite connectivities.

\subsection{Self-consistent effective field approach and Replica symmetry breaking}

We base the analysis of replica symmetry breaking of this section on the numerical investigations from section 2.3 and 3.2 , which consist of iterating the implicit equation (2.24) resulting from the self-consistent effective field approach of section 2.1.1. There, we calculated a new configuration of effective fields resulting from the old configuration. Then we were looking for convergence when repeatedly renewing the configuration. In that way we were trying to find the single stable state a replica symmetric spin glass can be in, irrespective of whether it is replica symmetric or not. The method in this sections extends this earlier method in that it explicitly tries to distinguish between finding and failing of convergence to a single stable solution of the configuration of effective fields. Therefore, we renew the configuration for a certain number of times (here: 500 times), even if the algorithm has already converged. Then we store the value of the order parameter $(\Delta h)^{2}$. We repeat this procedure of storing intermediate results. In this way we accumulate a sample of 60 intermediate results, from which we exclude the first ones, to allow for convergence of the algorithm, if possible. From the remaining intermediate values of $(\Delta h)^{2}$ we calculate the variance $\operatorname{var}_{\Delta h}$, which becomes the criterion of convergence. We expect $\operatorname{var}_{\Delta h}$ to display two differ- 
ent characteristic cases. In the first case, the configuration of effective fields converges to a single stable solution, which corresponds to replica symmetry (resp. independence of the initialization of the effective fields). In this case, the sample of intermediate results consists of very similar values of $(\Delta h)^{2}$ and $\operatorname{var}_{\Delta h}$ will be small. This is still true if the configuration we find is subject to rotations in each iteration step (see chapter 3), as the order parameter $(\Delta h)^{2}$ is still stable. In the second case, the algorithm does not find a single stable solution and $\operatorname{var}_{\Delta h}$ will be some orders of magnitude larger compared to the results in case of replica symmetry. We deduce that the second case corresponds to broken replica symmetry, where we have many stable solutions (or at least metastable solutions for finite $N$ ), which spread the values of $(\Delta h)^{2}$ over a non-vanishing range. The results can be found in figure 4.1 - 4.5 for various system sizes $N$, connectivities $k+1$ and maximal component numbers $m$. The error bars correspond to the resulting variance $\operatorname{var}_{\Delta h}$ of the intermediate results. Additionally plotted are the results for $m_{\mathrm{eff}}(T)$ from section 3.2. To illustrate the failing of convergence in these figures, we have rescaled the error bars with a factor $c \geq 1$ (which is constant for a given system) in such a way that a visible error bar corresponds to this failing. It is clear to see that the error bars become large when the effective number of components $m_{\text {eff }}(T)$ has crossed the maximal component number $m$. There are two exceptions from this rule resulting from numerical reasons. On the one hand, a very slow convergence comparable to the total number of iterations of the configuration of fields in the algorithm will result in a larger error bar than expected from replica symmetry. However, this exception will mainly occur slightly below $T_{c}$ as it follows from critical slowing down close to the critical temperature. Critical slowing down describes the phenomenon of an increasing time to equilibrate as the temperature approaches $T_{c}$ [53]. From the bare value of the estimated error these scenarios are undistinguishable. The plotted data do not show this behaviour. The second exception is that, although we may be in the broken replica symmetric region, it is still possible that the algorithm converges to a single state or at least a mixture of only a few "sufficiently similar" states resulting in a very small variance (small error bars) of the intermediate results. This is the case if the configuration is trapped in a single valley of the broken phase space. However, as the failing of convergence due to the existence of more than a single stable state is the dominating effect, the results show that there is a large- $m$ limit in the whole low temperature phase (even for Heisenberg spins close to the critical temperature, see figure 4.5).

How can we explain the breaking of replica symmetry, as presented in this section? We have seen that the lower the temperature, the more components are necessary to restore replica symmetry. As for lower temperatures the 

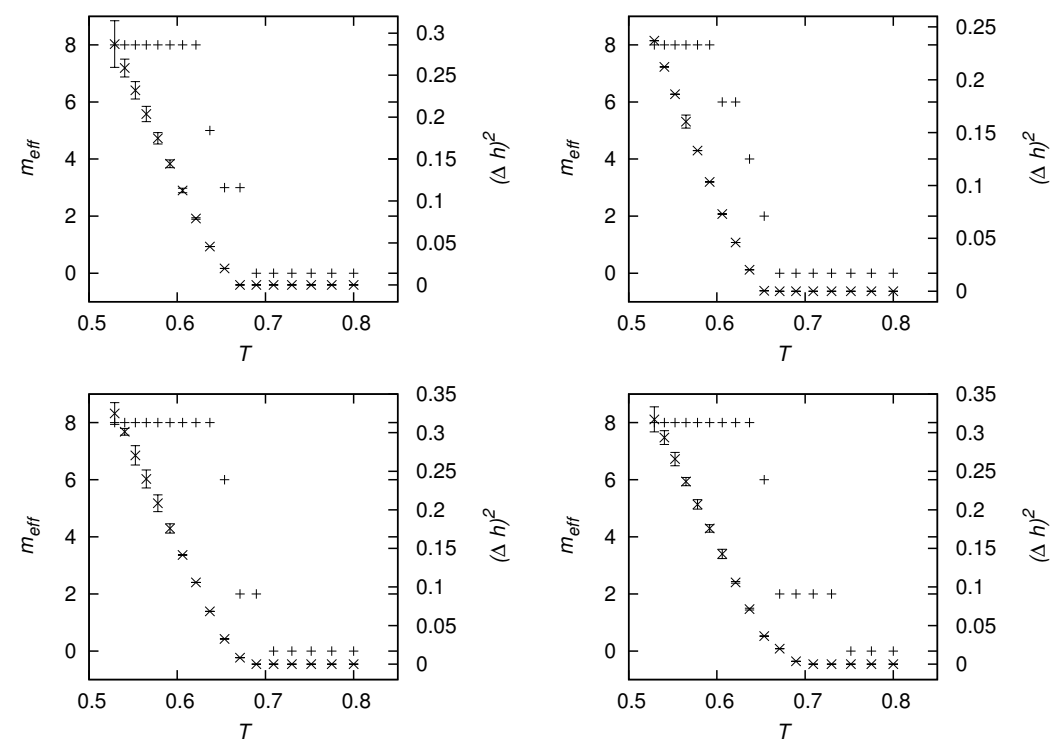

Figure 4.1: Plot of $m_{\mathrm{eff}}(T)$ (left axis, + ) and $(\Delta h)^{2}(\times)$ with error bars corresponding to $\operatorname{var}_{\Delta h}$ that indicate the failing of convergence (right axis) for $N=256, k+1=8$ and $m=8$. Replica symmetry is broken slightly below the temperature where $m_{\text {eff }}(T)$ crosses the maximal number of components available, $m$.
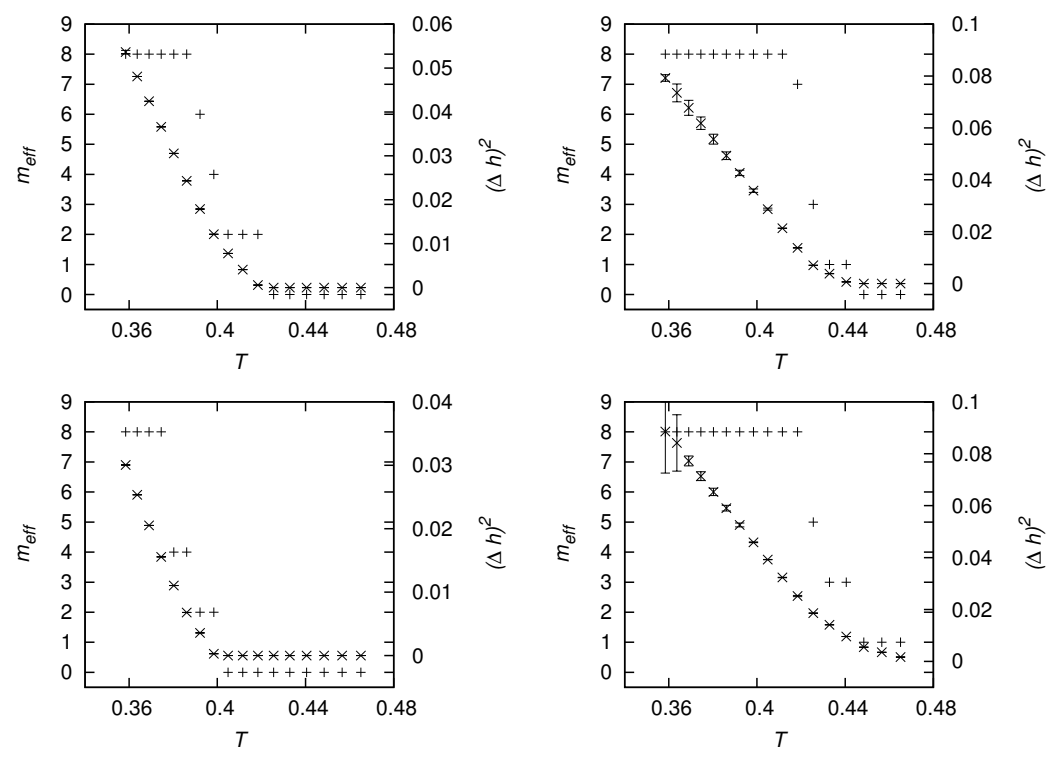

Figure 4.2: Corresponding plot to 4.1 for 256 spins with eight components on Bethe lattices with connectivity equal to four. 

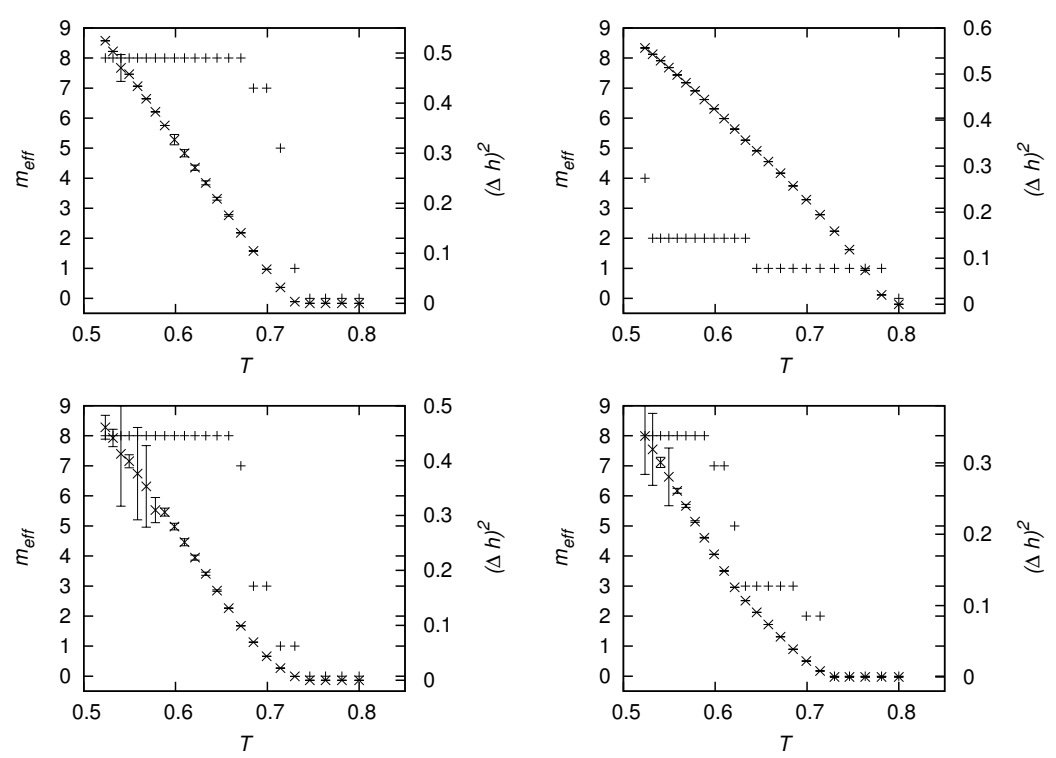

Figure 4.3: Corresponding plot to 4.1 for 96 spins with eight components on Bethe lattices with connectivity equal to eight.
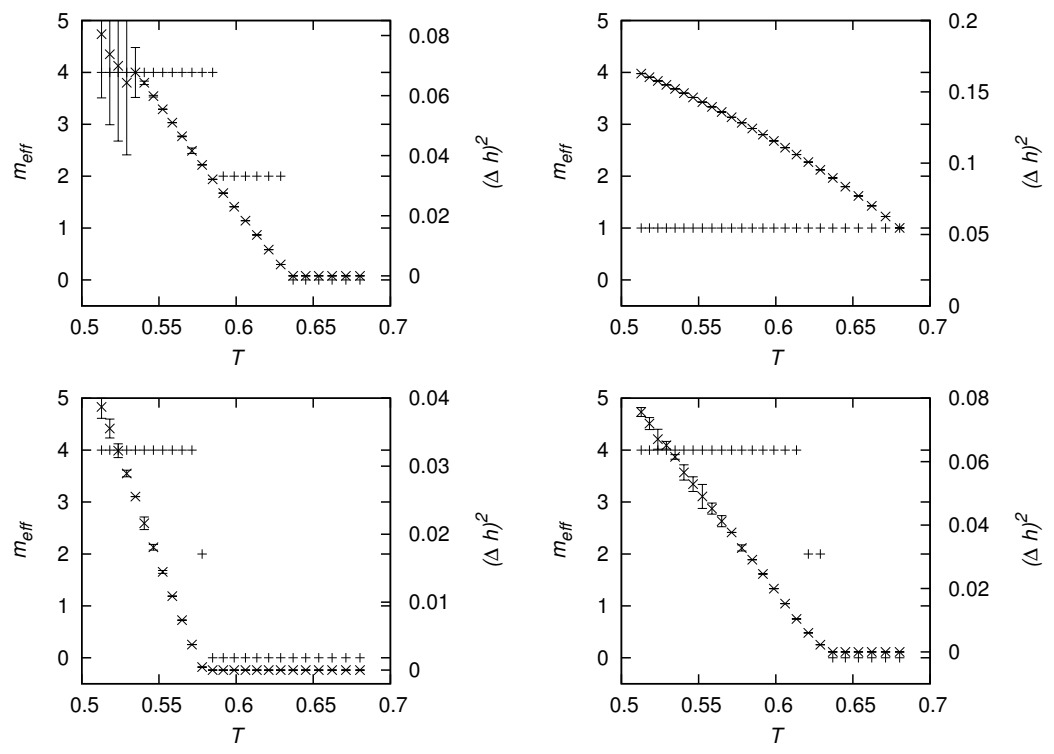

Figure 4.4: Corresponding plot to 4.1 for 512 spins with four components on Bethe lattices with connectivity equal to six. 

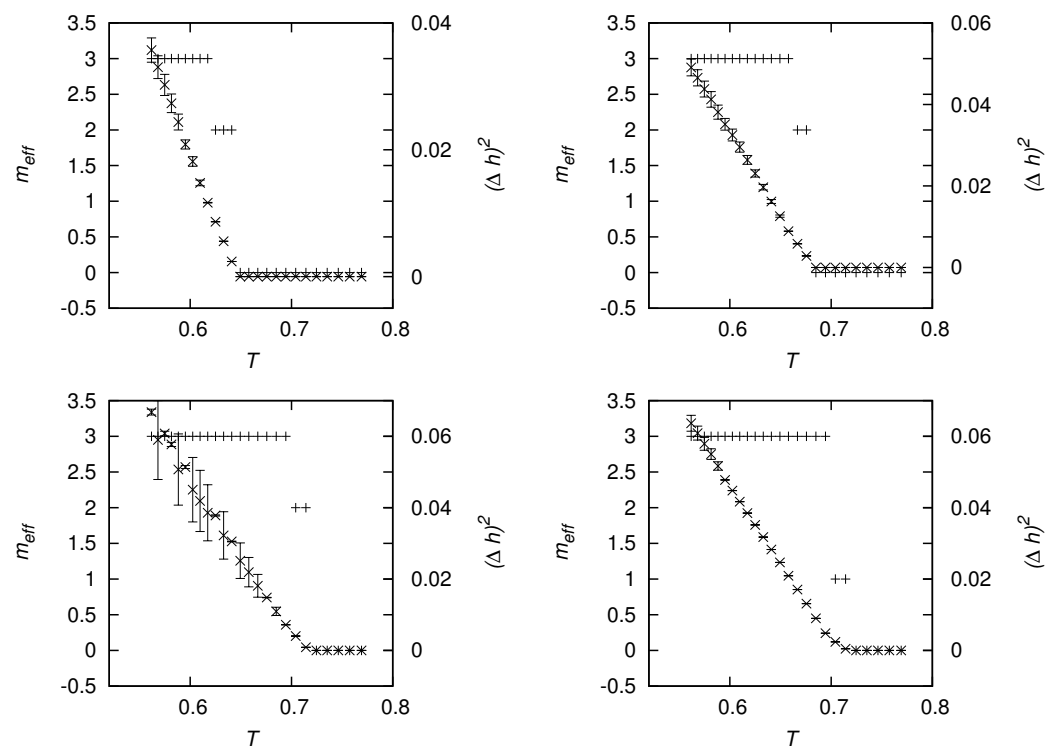

Figure 4.5: Corresponding plot to 4.1 for 128 spins with three components on Bethe lattices with connectivity equal to seven.

spins $\vec{s}_{i}$ are less affected by thermal fluctuations, the restriction to align to their mean local fields $\vec{H}_{i}$ grows stronger. Due to this, the degree of frustration grows and apparently more degrees of freedom (i.e. components) are needed to avoid the complex situation of highly frustrated systems leading to broken replica symmetry. To be precise, within the simulation we have detected rotations within each iteration step, see chapter 3 . These rotations are restricted to the available space, i.e. to the dimension of the spin space $m$. If, however, the fields already use all available components and a further rotation occurs (when lowering the temperature), which is at least partly perpendicular to the initial rotations, this rotation can not be described completely within the available $m$ components. What we observe in this case is simply a projection of this rotation onto the available $m$ dimensional space. This is not a stable configuration and leads to the failing of convergence, i.e. to the breaking of replica symmetry. We expect that, if there were enough components to describe the additional rotation, we would still find convergence of the algorithm (which is easy to understand as we would still be in the large- $m$ limit).

There are some interesting implications of this result. First of all we see that for every vector spin glass of finite size with $m \geq 2$, there is a (at least) a small range of temperatures below $T_{c}$ in which the system is replica symmetric. Only the Ising spin glass does in general not exhibit 
such a replica symmetric sector. Second, the results are consistent with the result of Ref. 41, where replica symmetry is broken for all finite $m$ in the thermodynamic limit. As we expect scaling of the effective number of components as in equation (3.6), i.e. as $m_{\text {eff }}(T) \sim N^{\mu_{m}}$, we should find broken replica symmetry for finite $m$ in the whole low temperature phase in the limit $N \rightarrow \infty$ as well. Third, the algorithm of iterating a configuration of effective fields allows us to discover the breaking of replica symmetry and confirms that it is a useful technique to investigate spin glasses on Bethe lattices.

\subsection{Parallel Tempering and Replica symme- try breaking}

In chapter 2 we have seen, that the parallel tempering Monte Carlo method did help us to observe the phase transition of the $m$-component spin glass on a Bethe lattice and to confirm the findings from the self-consistent effectivefield approach. The breaking of replica symmetry can be observed with the data from the parallel tempering simulations as well. To this end, we have to analyze the dependence of the variance of $q_{L}$ (instead of the mean), $\left(\Delta q_{L}\right)^{2}=\left[q_{L}^{2}\right]-\left[q_{L}\right]^{2}$, on the system size. This quantity allows to distinguish between replica symmetry and broken replica symmetry. The reason has been discussed in the introduction of this thesis: In case of replica symmetry, the link overlap between two copies of the same spin glass can only attain a single value, $q_{E A}$, in the thermodynamic limit, as there is only a single stable state the systems can be in. Therefore, the variance of the link overlap should vanish in the thermodynamic limit in that case, as $\left(\Delta q_{L}\right)^{2} \sim N^{-\alpha}$ with some unknown exponent $\alpha$ (however, we have to take the possible dependence on the component number $m$ into account). In contrast to that, in case of broken replica symmetry the link overlap distribution has support on a non-vanishing interval [8, 4] in the thermodynamic limit, as the systems can attain many stable states and the link overlap can take smaller values than $q_{E A}$. Therefore, the variance has a non-zero value in the thermodynamic limit.

Within the data we should find both cases, as we have seen in the previous section that for large component numbers $m\left(m>m_{\text {eff }}(T)\right)$ the system is replica symmetric, whereas replica symmetry is broken for $m<m_{\text {eff }}(T)$. We take all simulated component numbers from 2 to 6 and the various system sizes into consideration and calculate the link overlaps at the lowest temperature that is simulated. We indeed observe different scaling behaviour for 
$m>m_{\mathrm{eff}}(T)$ and $m<m_{\mathrm{eff}}(T)$ in Figure 4.6 , where we have plotted the variance of the link overlap $\left(\Delta q_{L}\right)^{2}$ (multiplied by $m$ ) with respect to the system size $N$ (multiplied by $m^{-1 / \mu_{m}}$, where $\mu_{m}=0.377$ in case of a Bethe lattice with a connectivity of eight). The scaling with the number of components $m$ makes the data independent of $m$, as they collapse and describe a single distribution. The final scaling result therefore is $m\left(\Delta q_{L}\right)^{2} \sim\left(N m^{-1 / \mu_{m}}\right)^{-\alpha}$. Even more, the chosen scaling of the $x$-axis as $N m^{-1 / \mu_{m}}$ allows to estimate the transition from replica symmetry to broken replica symmetry. As the system should be replica symmetric as long as $m \gg N^{\mu_{m}}$ (in the large- $m$ limit), we expect the transition to broken replica symmetry to happen for $m=\mathcal{O}\left(N^{\mu_{m}}\right)$, or $N m^{-1 / \mu_{m}}=\mathcal{O}(1)$, for all simulated component numbers $m$. This is precisely what we observe, though this transition can not be sharp due to the finite number of degrees of freedom of the systems. In the large- $m$ limit on the left hand side of Figure 4.6, we find a vanishing variance of the link overlap with the system size as $\left(\Delta q_{L}\right)^{2} \sim N^{-\alpha}$ (with the estimate of $\alpha \approx 1.4$ ) for all different values of $m$. On the right hand side we find the variance to approach a plateau (though this transition is very slow and it would require larger system sizes to see it more clearly), as predicted from broken replica symmetry. We remark that the scaling ansatz for $\left(\Delta q_{L}\right)^{2}$ is partly motivated from the computation following in the next chapter. As the ansatz will be used there as well, we refer to section 5.3.4 for further details.

In addition to earlier results from the parallel tempering Monte Carlo simulations in chapter 2 , the data from these simulations allow us to confirm the breaking of replica symmetry below the critical temperature for $m \ll$ $N^{\mu_{m}}$, resp. that replica symmetry holds in the large- $m$ limit. Even more, they support the finding of an effective number of components to build replica symmetric equilibrium states for finite system sizes. Although these results have been derived in other sections and chapters of this thesis, it is not at all self-evident to find them within the parallel tempering Monte Carlo simulations. The reason is that these simulations are subject to thermal fluctuations, whereas the simulation of the configuration of effective fields was not. The existence of the large- $m$ limit is one of the main results of this thesis and will be very useful in the last chapter. Furthermore, the parallel tempering Monte Carlo algorithm will be used there as well, with the difference that we will be interested in the infinite range model (the SK-model) of the $m$-component spin glass, instead of the Bethe lattice spin glass. 


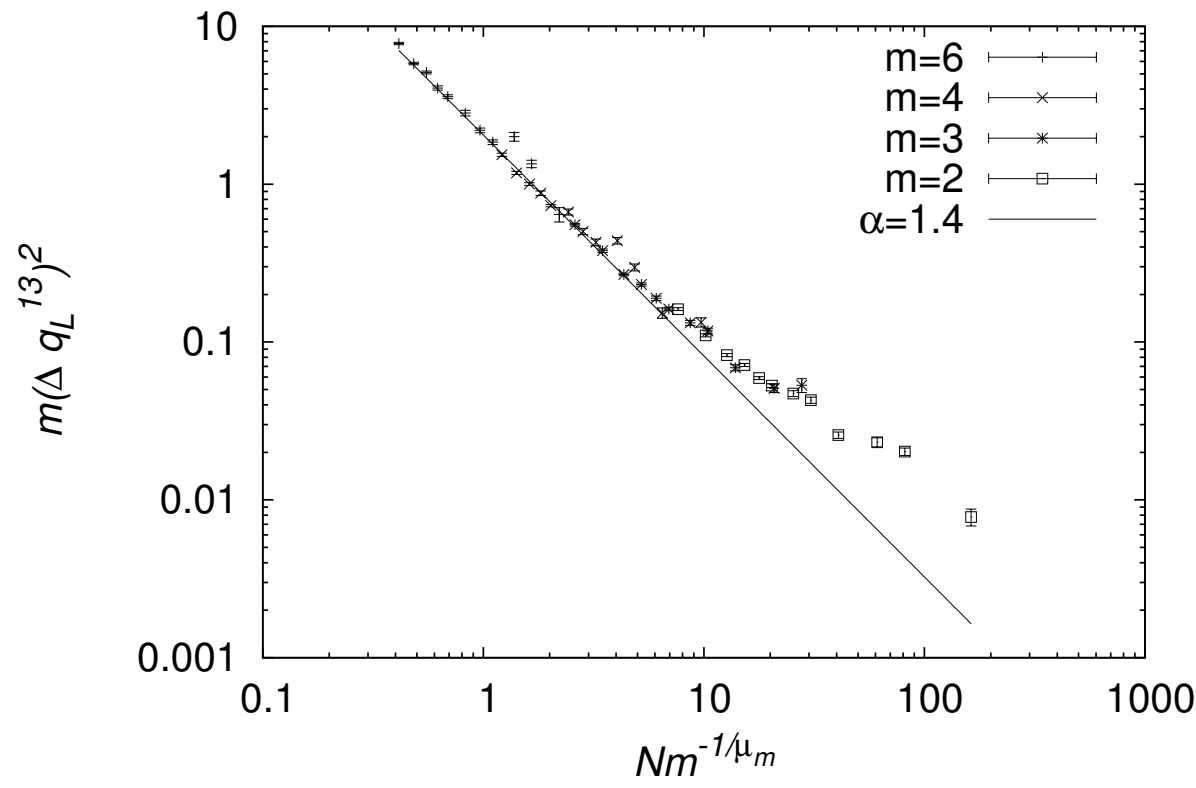

Figure 4.6: Finite size scaling plot (doubled logarithmic) of the variance of the link overlap at temperature $T=0.5$.

\subsection{Analytical investigation of Replica Sym- metry Breaking}

As shown in Ref. [41, replica symmetry is broken for all finite $m$ in the thermodynamic limit. In the previous sections we have seen that it is even broken for finite $N$ below the large- $m$ limit. However, so far there is only numerical support for this phenomenon. This section is an attempt to describe the breaking of replica symmetry below the large-m limit, which we have found numerically in the previous sections, in an analytical way. To this end, we will adapt a method developed by Goldschmidt and de Dominicis [72]. It is based on the derivation of a function $g_{n}^{k}(\vec{\sigma})$, using an iterative description of the partition function of a single spin $\vec{\sigma}$ on a Bethe lattice with connectivity $k+1$ (similar to the cavity method of chapter 2), which allows us to estimate corrections to the fully connected model in a power series in $\frac{1}{k}$. This function is used as the weight function (similar to a partition function) in the calculation of the overlaps $q_{\alpha \beta}^{\mu \nu}=\left\langle\sigma_{\alpha}^{\mu} \sigma_{\beta}^{\nu}\right\rangle$, where $\alpha, \beta=1, \cdots, n$ denote two replicas of the $n$-times replicated partition function and $\mu, \nu=1, \cdots, m$ are two of the $m$ available components. The calculation can be done with a replica symmetric ansatz for the overlaps or, alternatively with an one step broken 
replica symmetric ansatz (denoted by $1-\mathrm{RSB}$ as a short hand) which is a first step of introducing the complicated replica symmetry breaking scheme of the mean field Parisi solution [9, 10, 4]. Finally, it is possible to express the free energy in terms of these overlaps and to compare the results in order to decide which of the two alternatives minimizes the free energy. As the derivation of the weight function $g_{n}^{k}(\vec{\sigma})$ becomes exact only in the thermodynamic limit, we are restricted to introduce corrections for the number of components $m$ (instead of the system size $N$ ) to find the breaking of replica symmetry below the large- $m$ limit. These corrections occur when expanding the saddle point calculation of $g_{n}^{k}(\vec{\sigma})$ for $m \rightarrow \infty$ around the saddle point, as will be described in the following.

The overlaps $q_{\alpha \beta}^{\mu \nu}$ are the crucial quantities to distinguish replica symmetry and broken replica symmetry. To get used to their notation in the various cases (besides the distinction of replica symmetry and broken replica symmetry, we calculate the overlaps with corrections in $\frac{1}{m}$ up to $\frac{1}{m^{4}}$, with corrections in $\frac{1}{k}$ and without any corrections), they are explained already at this point. In each of the calculations we choose an ansatz which sets the overlaps to zero for $\mu \neq \nu$ and independent of the special choice of the investigated component (which reflects the isotropy of the model). Therefore we omit these indices as soon as possible after the definition of $q_{\alpha \beta}^{\mu \nu}$. In the replica symmetric case, we only have to distinguish whether the two replicas $\alpha$ and $\beta$ are identical or different. Therefore we have to find the overlaps $q_{2}$ (for identical replicas) and $q_{11}$ (for different replicas). In the 1-RSB case the $n$ replicas separate into $\frac{n}{r}$ blocks of $r$ replicas apiece. It is the first step of introducing the hierarchical structure of the phase space of the broken replica symmetry solution. In the thermodynamic limit this structure leads to a non ergodic phase space. In the case of 1 -RSB, the replica symmetric overlap $q_{11}$ separates into two different overlaps, $q_{11,2}$ (two spins of different replicas within the same block) and $q_{11,11}$ (two spins of different replicas in different blocks). As the replica method requires to take the limit $n \rightarrow 0$ in the end, the results of the 1-RSB ansatz will depend on the block size parameter $r$, and we will have to maximize the free energy with respect to it (this is a characteristic property of the replica method in spin glasses). Besides choosing a notation appropriate to describe replica symmetry and 1-RSB resp., it also has to answer the purpose of denoting the degree of the corrections involved. To this end, the final notation is $q_{n_{1}, n_{2}}^{(k m)}$, where $n_{1}$ specifies whether the two replicas the overlap is calculated of are different $\left(n_{1}=11\right)$ or identical $\left(n_{1}=2\right)$, and $n_{2}$ does the same for the "block" both replicas are taken from (the index $n_{2}$ will be omitted in the replica symmetric ansatz). The order of corrections to the fully connected model in the large- $m$ limit is denoted by $k$ (as the order of correction in $\frac{1}{k}$ ) and $m$ (as the order 
of correction in $\left.\frac{1}{m}\right)$. For instance, the overlap $q_{11,2}^{(02)}$ is the overlap from two different replicas taken from the same "replica-block", it is of order $\frac{1}{k^{0}}$ in the connectivity (i.e. fully connected) and the corrections to the saddle point for $m \rightarrow \infty$ are of order $\frac{1}{m^{2}}$. If the overlap involves no corrections to the saddle point in any power of $\frac{1}{m}$ we omit this subscript and write $q_{n_{1}, n_{2}}^{(k)}$. In addition to the two-spin overlaps $q_{\alpha \beta}^{\mu \nu}=\left\langle\sigma_{\alpha}^{\mu} \sigma_{\beta}^{\nu}\right\rangle$, the free energy involves fourspin overlaps $q_{\alpha \beta \gamma \delta}^{\mu \nu \eta \rho}=\left\langle\sigma_{\alpha}^{\mu} \sigma_{\beta}^{\nu} \sigma_{\gamma}^{\eta} \sigma_{\delta}^{\rho}\right\rangle$. Their notation is similar to the notation of the two-spin overlaps, with the sole difference that $n_{1}$ and $n_{2}$ denote the distribution of four spins over different replicas and replica-blocks.

We see that the interest in this calculation is twofold. On the one hand, we are going to introduce corrections to the large- $m$ limit and ask whether replica symmetry still holds for finite $m$. On the other hand we introduce corrections to the fully connected model, i.e. we describe a finite connectivity and therefore a different picture of neighbourhood of the spins as in the SKmodel. To facilitate following the framework of this chapter, we give the final results already at this stage. First of all, without any corrections, we find the $m$-component spin glass to be replica symmetric, as in Ref. [41]. The only solution the 1-RSB ansatz exhibits is $q_{11,2}^{(0)}=q_{11,11}^{(0)}$, i.e. identical to the replica symmetric solution. Generally, when we want to calculate the correction terms in $\frac{1}{m}$, we will see that we have to know the solutions of all lower orders than the one we are interested in. The reason for this is a Taylor expansion around the zeroth order solution (i.e. for $m \rightarrow \infty$ ). With it, we find a set of hierarchical equations, which we can solve subsequently beginning with the first order. In case of replica symmetry we can calculate the overlaps up to fourth order in $\frac{1}{m}$ explicitly. In the case of 1-RSB, we find the solution in first order from the equations determining $q_{11, x}^{(02)}(x=2,11)$, i.e. the equations in second order, for the reason that they reduce the solution space we find in first order. We will see why this is the case. The solution in second order is itself found from the equations determining $q_{11, x}^{(04)}$ for the same reason. Both solutions are replica symmetric, i.e. up to this order we do not find the breaking of replica symmetry we are searching for. The equations in third and fourth order, which we have to set up in order to find the solution in second order, are not sufficient to decide whether replica symmetry is broken or not, but one would have to go to even higher orders to determine these solutions. Finally, we will discuss why replica symmetry breaking is not found, and how one could proceed to observe it.

Concerning the corrections in $\frac{1}{k}$ we can calculate the replica symmetric solution, $q_{11}^{(1)}$, and find a simple relation in case of 1 -RSB, $(r-1) q_{11,2}^{(1)}=$ $r q_{11,11}^{(1)}+c(T)$, which is consistent with the replica symmetric result for $r=0$ or $r=1$. To decide whether replica symmetry is broken we have to maximize 
the contributions to the free energy in order $\frac{1}{k^{2}}$ (for reasons which will become apparent below). We find that the free energy is independent of the choice of $r$, i.e. the free energy is flat along the 1-RSB mode. We will discuss implications of this result.

\subsubsection{Method / Basic Formalism}

The method used can be summarized in a few steps. First of all we derive the weight function $g_{n}(\vec{\sigma})$ by describing the partition function of the central spin $\vec{\sigma}_{0}$ on a Cayley tree iteratively and explain how it can be used on a Bethe lattice. Afterwards we define the overlaps $q_{\alpha \beta}^{\mu \nu}$, which emerge when parametrizing $g_{n}(\vec{\sigma})$ in an alternative way and therefore are determined by $g_{n}(\vec{\sigma})$ (this is the weight function with respect to which the overlaps are calculated). Then we express the free energy in terms of $g_{n}(\vec{\sigma})$ and the overlaps $q_{\alpha \beta}^{\mu \nu}$. Finally we calculate the overlaps and the free energy with a replica symmetric and an 1-RSB ansatz resp., and compare the results.

We start by considering the $m$-component spin glass on a Cayley tree, i.e. a tree without loops, which has a central spin $\vec{\sigma}_{0}$ that is connected to $k+1$ branches of the tree. The tree has $z$ shells and each shell consists of spins that have one neighbour on its neighbouring inner shell and $k$ neighbouring spins on the neighbouring outer shell. The spins on the last shell, i.e. on the boundary, have only one neighbour (that belongs to the penultimate shell). The Bethe lattice instead is locally equal to a Cayley tree, with the difference that it has no boundaries, but that every spin is connected with the same fixed number of nearest neighbours, see chapter 2. The $n$ times replicated partition function for the central spin $\vec{\sigma}_{0}$ on a Cayley tree is

$$
Z^{n}=\operatorname{Tr}_{\sigma} \exp \left(\beta \sum_{\alpha=1}^{n} \sum_{\langle i j\rangle} J_{i j}{\overrightarrow{\sigma_{i}}}^{\alpha}{\overrightarrow{\sigma_{j}}}^{\alpha}\right) \text {. }
$$

If we denote the $k+1$ neighbouring spins of $\vec{\sigma}_{0}$ by $\vec{s}^{(l)}$ with $l=1, \cdots, k+1$, and the $k$ other neighbouring spins of each of the spins $\vec{s}^{(l)}$ by $\vec{t}$ we can proceed iteratively by writing

$$
\begin{aligned}
Z^{n} & =\operatorname{Tr}_{\sigma} \prod_{l=1}^{k+1} \prod_{\alpha=1}^{n} Q_{(z)}\left({\overrightarrow{\sigma_{0}}}^{\alpha} \mid \vec{s}^{(l) \alpha}\right) \quad, \text { with } \\
Q_{(z)}\left({\overrightarrow{\sigma_{0}}}^{\alpha} \mid \vec{s}^{\alpha}\right) & =\exp \left(\beta J_{s 0}{\overrightarrow{\sigma_{0}}}^{\alpha} \vec{s}^{\alpha}\right) \prod_{l=1}^{k} Q_{(z-1)}\left(\vec{s}^{\alpha} \mid \vec{t}^{l l) \alpha}\right),
\end{aligned}
$$

where the argument $\vec{\sigma}_{0} \mid \vec{s}$ denotes the spin $\vec{\sigma}_{0}$ in dependence of the direction of $\vec{s}$ and the index of $Q$ denotes the number of remaining shells it consid- 
ers, while $Q$ is the conditional probability distribution of finding a certain direction of $\vec{\sigma}_{0}$, given $\vec{s}$. The link between $\vec{\sigma}_{0}$ and $\vec{s}$ is denoted by $J_{s 0}$. In the iterative sense of formulating the partition function in equation 4.2), we introduce the function $g_{(z)}(\vec{\sigma})$ as the partition function of $n$ replicas of any arbitrary single spin on any of the $z$ shells (here for the central spin $\vec{\sigma}_{0}$ ) interacting solely with a single branch of the remaining outer shells of the Cayley tree

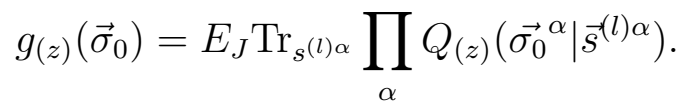

Iteratively it follows

$$
E_{J} Z^{n}=\operatorname{Tr}_{\sigma_{0}} g_{(z)}^{k+1}\left(\vec{\sigma}_{0}\right)
$$

where $g_{(z)}^{k+1}\left(\vec{\sigma}_{0}\right)$ describes the interaction of $\vec{\sigma}_{0}$ with all of its $k+1$ neighbouring spins, which is denoted by the superscript $k+1$. Furthermore, we can express $g_{(z)}\left(\vec{\sigma}_{0}\right)$ self-consistently (only writing the interaction between $\vec{\sigma}_{0}$ and its neighbour $\vec{s}_{1}$ explicitly and subsume the remaining interactions in $\left.g_{(z-1)}^{k}\left(\vec{s}_{1}\right)\right)$

$$
g_{(z)}\left(\vec{\sigma}_{0}\right)=E_{J} \operatorname{Tr}_{s_{1}^{\alpha}} \exp \left(\beta J \sum_{\alpha}{\overrightarrow{\sigma_{0}}}^{\alpha}{\overrightarrow{s_{1}}}^{\alpha}\right) g_{(z-1)}^{k}\left(\vec{s}_{1}\right) .
$$

In the thermodynamic limit these equations hold on the Bethe lattice, because of its locally tree like structure. The Bethe lattice is more appropriate to investigate a spin glass as the Cayley tree, as it has no boundaries, but large loops that introduce frustration. Therefore we will substitute the index $z$ of $g_{(z)}$ (an enumeration of the shells is not possible on a Bethe lattice) in favour of $g_{n}$, which is the corresponding function on the Bethe lattice, in the next sections ( $n$ denotes the $n$-times replicated partition function). With an appropriate normalisation $\left(\operatorname{Tr}_{s_{1}} g_{n}^{k}\left(\vec{s}_{1}\right)\right)^{(-1)}$ from Ref. [72] and the generalization of the spins $\vec{\sigma}_{0}^{\alpha} \rightarrow \vec{\sigma}_{\alpha}$ and $\vec{s}_{1}^{\alpha} \rightarrow \vec{\tau}_{\alpha}$, we finally have

$$
g_{n}(\vec{\sigma})=E_{J} \operatorname{Tr}_{\tau} \exp \left(\beta J \sum_{\alpha=1}^{n} \vec{\sigma}_{\alpha} \vec{\tau}_{\alpha}\right) g_{n}^{k}(\vec{\tau})\left(\operatorname{Tr}_{\tau} g_{n}^{k}(\vec{\tau})\right)^{-1}
$$

Additionally to equation (4.6) we can express $g_{n}(\vec{\sigma})$ in terms of the overlaps $q_{\alpha \beta}^{\mu \nu}$. To find this formulation, we scale the coupling as $\frac{J}{\sqrt{k}}$, expand the exponential in equation 4.6 in a power series and get

$$
g_{n}(\vec{\sigma})=\sum_{r=0}^{\infty} E_{J} \frac{1}{r !}\left(\frac{\beta J}{\sqrt{k}}\right)^{r} \sum_{\substack{\left(\alpha_{1}, \ldots, \alpha_{r}\right) \\ \mu_{1}, \ldots, \mu_{r}}} \operatorname{Tr}_{\tau} \tau_{\alpha_{1}}^{\mu_{1}} \sigma_{\alpha_{1}}^{\mu_{1}} \ldots \tau_{\alpha_{r}}^{\mu_{r}} \sigma_{\alpha_{r}}^{\mu_{r}} g_{n}^{k}(\vec{\tau})\left(\operatorname{Tr}_{\tau} g_{n}^{k}(\vec{\tau})\right)^{-1}
$$


where $\alpha_{1}, \ldots, \alpha_{r}$ denote the $r$ possibly different replicas and $\mu_{1}, \ldots, \mu_{r}$ denote the $r$ possibly different components in each sum. We define the overlap parameters $q_{\alpha_{1} \ldots \alpha_{r}}^{\mu_{1} \ldots \mu_{r}}$ in the following way

$$
q_{\alpha_{1} \ldots \alpha_{r}}^{\mu_{1} \ldots \mu_{r}}=\operatorname{Tr}_{\tau} \tau_{\alpha_{1}}^{\mu_{1}} \ldots \tau_{\alpha_{r}}^{\mu_{r}} \frac{g_{n}^{k}(\vec{\tau})}{\operatorname{Tr}_{\tau} g_{n}^{k}(\vec{\tau})}
$$

and insert them into equation 4.7), which becomes a series in powers of $\frac{1}{\sqrt{k}}$

$$
g_{n}(\vec{\sigma})=1+\frac{\lambda^{2}}{2 k} \sum_{\substack{\alpha, \beta \\ \mu, \nu}} q_{\alpha \beta}^{\mu \nu} \sigma_{\alpha}^{\mu} \sigma_{\beta}^{\nu}+\frac{\lambda^{4}}{24 k^{2}} \sum_{\substack{\alpha, \beta, \gamma, \delta \\ \mu, \nu, \eta, \rho}} q_{\alpha \beta \gamma \delta}^{\mu \nu \eta \rho} \sigma_{\alpha}^{\mu} \sigma_{\beta}^{\nu} \sigma_{\gamma}^{\eta} \sigma_{\delta}^{\rho}+\mathcal{O}\left(\frac{1}{k^{3}}\right) .
$$

Here, we used a symmetric $\pm J$-distribution for averaging over the disorder $E_{J}$ (note that the prefactor $\frac{1}{24}$ becomes equal to $\frac{1}{8}$ for Gaussian distributed $J)$. The odd moments of such a symmetric distribution vanish. With a variance of 1 for this distribution, we end up in the last line, with $\lambda=\beta$.

In addition to equation (4.9), we derive a second useful expansion of $g_{n}^{k}$ (by a separation of the overlap parameters of the form $q_{\alpha \beta}^{\mu \nu}=q_{\alpha \beta}^{\mu \nu(0)}+\frac{1}{k} q_{\alpha \beta}^{\mu \nu(1)}$ (i.e. into the leading part with $k \rightarrow \infty$ and corrections in $\frac{1}{k}$ ), further details in the appendix B.1)

$$
\begin{aligned}
g_{n}^{k}(\vec{\sigma}) & =\exp \left(k \ln \left(1+\frac{\lambda^{2}}{2 k} \sum_{\substack{\alpha, \beta \\
\mu, \nu}} q_{\alpha \beta}^{\mu \nu} \sigma_{\alpha}^{\mu} \sigma_{\beta}^{\nu}+\frac{\lambda^{4}}{24 k^{2}} \sum_{\substack{\alpha, \beta, \gamma, \delta \\
\mu, \nu, \eta, \rho}} q_{\alpha \beta \gamma \delta}^{\mu \nu \eta \rho} \sigma_{\alpha}^{\mu} \sigma_{\beta}^{\nu} \sigma_{\gamma}^{\eta} \sigma_{\delta}^{\rho}\right)\right) \\
& =\exp \left(\frac{\lambda^{2}}{2} \sum_{\substack{\alpha, \beta \\
\mu, \nu}} q_{\alpha \beta}^{\mu \nu(0)} \sigma_{\alpha}^{\mu} \sigma_{\beta}^{\nu}\right)\left(1+\frac{\lambda^{2}}{2 k} \sum_{\substack{\alpha, \beta \\
\mu, \nu}} q_{\alpha \beta}^{\mu \nu(1)} \sigma_{\alpha}^{\mu} \sigma_{\beta}^{\nu}+\frac{\lambda^{4}}{24 k} \sum_{\substack{\alpha, \beta, \gamma, \delta \\
\mu, \nu, \eta, \rho}} q_{\alpha \beta \gamma \delta}^{\mu \nu \eta \rho(0)} \sigma_{\alpha}^{\mu} \sigma_{\beta}^{\nu} \sigma_{\gamma}^{\eta} \sigma_{\delta}^{\rho}\right. \\
& \left.-\frac{\lambda^{4}}{8 k}\left(\sum_{\substack{\alpha, \beta \\
\mu, \nu}} q_{\alpha \beta}^{\mu \nu(0)} \sigma_{\alpha}^{\mu} \sigma_{\beta}^{\nu}\right)^{2}+\mathcal{O}\left(\frac{1}{k^{2}}\right)\right)
\end{aligned}
$$

\section{Convenient form of the free energy}

In the appendix B.2 we derive a convenient expression for the free energy of the $m$ component spin glass on a Bethe lattice in terms of $g_{n}$, previously derived in Ref. [72] for Ising spins. The final result for the free energy per spin, $f=F / N$, is

$$
\begin{aligned}
n \beta f & =k \ln \operatorname{Tr}_{\sigma} g_{n}^{k+1}(\vec{\sigma}) \\
& -\frac{k+1}{2} \ln E_{J} \operatorname{Tr}_{\tau} \exp \left(\beta J \sum_{\alpha=1}^{n} \vec{\sigma}_{\alpha} \vec{\tau}_{\alpha}\right) g_{n}^{k}(\vec{\tau}) g_{n}^{k}(\vec{\sigma}) .
\end{aligned}
$$

This formulation of the free energy will be used in the following. 


\section{The free energy in terms of the overlaps $q_{\alpha \beta}^{\mu \nu}$}

The overlap parameters $q_{\alpha \beta}^{\mu \nu}$ are the crucial quantities to distinguish replica symmetry and 1-RSB. They are calculated by using $g_{n}(\vec{\sigma})$ as a weight function. We want to express the free energy in terms of the overlaps. To this end, we insert the result of equation (4.9) into equation (4.11). Then we separate it into two parts $f=f_{0}+\frac{1}{k} f_{1}+\ldots$ by separating the overlap parameters $q_{\alpha \beta}^{\mu \nu}=q_{\alpha \beta}^{\mu \nu(0)}+\frac{1}{k} q_{\alpha \beta}^{\mu \nu(1)}$ analogously, and similarly for $q_{\alpha \beta \gamma \delta}^{\mu \nu \eta \rho}$. For the details of the calculation we refer to the appendix B.3. In summary, the free energy consists of the following parts (up to order $\frac{1}{k}$ ):

$$
\begin{aligned}
m n \beta f_{0} & =\frac{\lambda^{2}}{4} \sum_{\substack{\alpha, \beta \\
\mu, \nu}}\left(q_{\alpha \beta}^{\mu \nu(0)}\right)^{2}-\ln \operatorname{Tr} \exp \left(\frac{\lambda^{2}}{2} \sum_{\substack{\alpha, \beta \\
\mu, \nu}} q_{\alpha \beta}^{\mu \nu(0)} \sigma_{\alpha}^{\mu} \sigma_{\beta}^{\nu}\right) \\
m n \beta f_{1} & =-\frac{\lambda^{2}}{4} \sum_{\substack{\alpha, \beta \\
\mu, \nu}}\left(q_{\alpha \beta}^{\mu \nu(0)}\right)^{2}-\frac{\lambda^{4}}{48} \sum_{\substack{\alpha, \beta, \gamma, \delta \\
\mu, \nu, \eta, \rho}}\left(q_{\alpha \beta \gamma \delta}^{\mu \nu \eta \rho(0)}\right)^{2} \\
& +\frac{\lambda^{4}}{8} \sum_{\substack{\alpha, \beta, \gamma, \delta \\
\mu, \nu, \eta, \rho}} q_{\alpha \beta}^{\mu \nu(0)} q_{\gamma \delta}^{\eta \rho(0)} q_{\alpha \beta \gamma \delta}^{\mu \nu \eta \rho(0)} .
\end{aligned}
$$

Note that the terms in $q_{\alpha \beta}^{\mu \nu(1)}$ have cancelled out and contribute in second order of the free energy $f_{2}$ for the first time. We further see that we only have to take the leading order in $n$ of the right hand side into account. As the sums are of order $m n$ as well as $\ln \operatorname{Tr} \exp \left(\frac{\lambda^{2}}{2} \sum_{, \mu \nu}^{\alpha, \beta} q_{\alpha \beta}^{\mu \nu(0)} \sigma_{\alpha}^{\mu} \sigma_{\beta}^{\nu}\right)$ (as will turn out in the next section), evaluating the overlaps in zeroth order in $n$ is sufficient.

\subsubsection{Resulting overlaps for $m, k \rightarrow \infty$ and corrections in $\frac{1}{m}$}

The equations 4.12 show the dependence of the free energy on the various overlaps $q_{\alpha \beta}^{\mu \nu(0)}$ and $q_{\alpha \beta \gamma \delta}^{\mu \nu \eta \rho(0)}$ and on the term $T=\operatorname{Tr} \exp \left(\frac{\lambda^{2}}{2} \sum_{\substack{\alpha, \beta \\ \mu, \nu}} q_{\alpha \beta}^{\mu \nu(0)} \sigma_{\alpha}^{\mu} \sigma_{\beta}^{\nu}\right)$. Inserting equation 4.10 into equation 4.8 we see that we have to use $T$ additionally to calculate the various overlaps in zeroth order in $\frac{1}{k}$ according to $q_{\alpha \beta}^{\mu \nu(0)}=\frac{2}{\lambda^{2}} \frac{d}{d q_{\alpha \beta}^{\mu \nu}} \ln T$. Since the overlaps are the key quantities to distinguish between replica symmetry and broken replica symmetry, it is necessary to calculate $T$ both in the replica symmetric and, as a first step towards full $\mathrm{RSB}$, in the $1-\mathrm{RSB}$ case. As replica symmetry should be broken in order $\frac{1}{\mathrm{~m}^{2}}$, according to Ref. [41, we introduce the general framework of expanding $T$ 
towards higher orders in $\frac{1}{m}$ and calculate the corrections up to the necessary order of four (which answers the question of replica symmetry breaking for the order $\frac{1}{m^{2}}$, as will become clear below). For this, we do not need to know the corrections in $\frac{1}{k}$, as the result of Ref. [41] is restricted to the SK-model.

In the following, we write the various overlaps $q$ as $q^{(0)}=q^{(00)}+\frac{1}{m} q^{(01)}+$ $\frac{1}{m^{2}} q^{(02)}+\frac{1}{m^{3}} q^{(03)}+\frac{1}{m^{4}} q^{(04)}$, and use a proper formalism to Taylor expand around the zeroth order results $q^{(00)}$ to derive the determining equations for the different orders of $q^{(0)}$. Details of the computation are given in the appendix B.3.1. The final result for $\ln T$ has the form (up to fourth order)

$$
\begin{aligned}
\ln T=m n\left(\epsilon-z-\frac{1}{2 n} \operatorname{Tr} \ln \left(M_{0}\right)\right. & -\frac{1}{2 n m} \ln \operatorname{Tr}\left(M_{0}^{-1}\right)^{2}+\frac{1}{n m^{2}} \ln T_{2} \\
& \left.+\frac{1}{n m^{3}} \ln T_{3}+\frac{1}{n m^{4}} \ln T_{4}+\ldots\right),
\end{aligned}
$$

with the explicit expressions

$$
\begin{aligned}
& \ln T_{2}=\frac{3 \operatorname{Tr}\left(M_{0}^{-1}\right)^{4}}{\left(\operatorname{Tr}\left(M_{0}^{-1}\right)^{2}\right)^{2}}+\frac{10\left(\operatorname{Tr}\left(M_{0}^{-1}\right)^{3}\right)^{2}}{3\left(\operatorname{Tr}\left(M_{0}^{-1}\right)^{2}\right)^{3}} \\
& \ln T_{3}=\frac{20 \operatorname{Tr}\left(M_{0}^{-1}\right)^{6}}{\left(\operatorname{Tr}\left(M_{0}^{-1}\right)^{2}\right)^{3}}+\frac{56 \operatorname{Tr}\left(M_{0}^{-1}\right)^{5} \operatorname{Tr}\left(M_{0}^{-1}\right)^{3}}{\left(\operatorname{Tr}\left(M_{0}^{-1}\right)^{2}\right)^{4}}+\frac{69\left(\operatorname{Tr}\left(M_{0}^{-1}\right)^{4}\right)^{2}}{4\left(\operatorname{Tr}\left(M_{0}^{-1}\right)^{2}\right)^{4}} \\
& +\frac{85 \operatorname{Tr}\left(M_{0}^{-1}\right)^{4}\left(\operatorname{Tr}\left(M_{0}^{-1}\right)^{3}\right)^{2}}{\left(\operatorname{Tr}\left(M_{0}^{-1}\right)^{2}\right)^{5}}+\frac{285\left(\operatorname{Tr}\left(M_{0}^{-1}\right)^{3}\right)^{4}}{9\left(\operatorname{Tr}\left(M_{0}^{-1}\right)^{2}\right)^{6}} \\
& \ln T_{4}=\frac{105 \operatorname{Tr}\left(M_{0}^{-1}\right)^{8}}{\left(\operatorname{Tr}\left(M_{0}^{-1}\right)^{2}\right)^{4}}+\frac{360 \operatorname{Tr}\left(M_{0}^{-1}\right)^{7} \operatorname{Tr}\left(M_{0}^{-1}\right)^{3}}{\left(\operatorname{Tr}\left(M_{0}^{-1}\right)^{2}\right)^{5}}+\frac{225 \operatorname{Tr}\left(M_{0}^{-1}\right)^{6} \operatorname{Tr}\left(M_{0}^{-1}\right)^{4}}{\left(\operatorname{Tr}\left(M_{0}^{-1}\right)^{2}\right)^{5}} \\
& +\frac{1512\left(\operatorname{Tr}\left(M_{0}^{-1}\right)^{5}\right)^{2}}{5\left(\operatorname{Tr}\left(M_{0}^{-1}\right)^{2}\right)^{5}}+\frac{3681\left(\operatorname{Tr}\left(M_{0}^{-1}\right)^{4}\right)^{3}}{8\left(\operatorname{Tr}\left(M_{0}^{-1}\right)^{2}\right)^{6}}+\frac{3820 \operatorname{Tr}\left(M_{0}^{-1}\right)^{6}\left(\operatorname{Tr}\left(M_{0}^{-1}\right)^{3}\right)^{2}}{9\left(\operatorname{Tr}\left(M_{0}^{-1}\right)^{2}\right)^{6}} \\
& +\frac{274 \operatorname{Tr}\left(M_{0}^{-1}\right)^{5} \operatorname{Tr}\left(M_{0}^{-1}\right)^{4} \operatorname{Tr}\left(M_{0}^{-1}\right)^{3}}{\left(\operatorname{Tr}\left(M_{0}^{-1}\right)^{2}\right)^{6}}+\frac{1302 \operatorname{Tr}\left(M_{0}^{-1}\right)^{5}\left(\operatorname{Tr}\left(M_{0}^{-1}\right)^{3}\right)^{3}}{3\left(\operatorname{Tr}\left(M_{0}^{-1}\right)^{2}\right)^{7}} \\
& +\frac{6925\left(\operatorname{Tr}\left(M_{0}^{-1}\right)^{4} \operatorname{Tr}\left(M_{0}^{-1}\right)^{3}\right)^{2}}{24\left(\operatorname{Tr}\left(M_{0}^{-1}\right)^{2}\right)^{7}}+\frac{8655 \operatorname{Tr}\left(M_{0}^{-1}\right)^{4}\left(\operatorname{Tr}\left(M_{0}^{-1}\right)^{3}\right)^{4}}{3\left(\operatorname{Tr}\left(M_{0}^{-1}\right)^{2}\right)^{8}} \\
& +\frac{67485\left(\operatorname{Tr}\left(M_{0}^{-1}\right)^{3}\right)^{6}}{81\left(\operatorname{Tr}\left(M_{0}^{-1}\right)^{2}\right)^{9}}
\end{aligned}
$$

The matrix $M_{0}$ has the entries $M_{0 \alpha \beta}^{\mu \nu}=2\left(\epsilon-z_{\alpha}\right) \delta_{\alpha \beta}-\lambda^{2} q_{\alpha \beta}^{\mu \nu}$, with the parameters $z_{\alpha}$ resulting from the integral representation of the constraint ${\overrightarrow{\sigma_{\alpha}}}^{2}=m$ and $\epsilon$ to ensure convergence of Gaussian integrals while evaluating the Trace over the spins. It is introduced in the appendix B.3.1 in more detail. The result of taking the Trace of any of the various powers of the $m n \times m n$ matrix 
$M_{0}^{-1}$ is of order $m n$, hence $\ln T$ is of the necessary order in $n$. In the following we denote the corresponding terms for the replica symmetric and the 1-RSB-case as $T_{t}^{R S}$ and $T_{t}^{1 R S B}$ resp., with $t=0, \ldots, 4$. From the derivation of these results, it should be clear that all of these terms depend solely on the overlaps $q_{\alpha \beta}^{\mu \nu}$. Now, we start with the calculation of the overlaps in zeroth order $(m \rightarrow \infty)$ in the replica symmetric case.

\section{Replica symmetry}

In the replica symmetric case we have two different possible overlaps to calculate, $\left\langle\sigma_{\alpha}^{\mu} \sigma_{\alpha}^{\nu}\right\rangle$ and $\left\langle\sigma_{\alpha}^{\mu} \sigma_{\beta}^{\nu}\right\rangle$. Due to isotropy of the $m$ component spin glass model, overlaps of different components $\mu$ and $\nu$ are set to zero [44]. This can be explicitly shown by calculating $q_{\alpha \beta}^{\mu \nu}$ using spherical coordinates, see appendix B.4. There we also see that a four-spin overlap is equal to zero if a certain component from 1 to $m$ occurs only once (or for an odd number, in general). Therefore, with the notation $q_{2}=\left\langle\sigma_{\alpha}^{\mu} \sigma_{\alpha}^{\mu}\right\rangle$ and $q_{11}=\left\langle\sigma_{\alpha}^{\mu} \sigma_{\beta}^{\mu}\right\rangle$, we have the following ansatz for the sums of the overlaps $q_{\alpha \beta}^{\mu \nu}$ and $q_{\alpha \beta \gamma \delta}^{\mu \nu \eta \rho}$

$$
\begin{aligned}
\sum_{\substack{\alpha, \beta \\
\mu, \nu}} q_{\alpha \beta}^{\mu \nu} & =\sum_{\substack{\alpha \neq \beta \\
\mu, \nu}} q_{11} \delta_{\mu \nu}+\sum_{\alpha, \mu} q_{2} \delta_{\mu \nu} \\
& =m n(n-1) q_{11}+m n q_{2} \\
\sum_{\substack{\alpha, \beta, \gamma, \delta \\
\mu, \nu, \rho, \rho}} q_{\alpha \beta \gamma \delta}^{\mu \nu \eta \rho} & =m n\left[(n-1)(n-2)(n-3)\left(q_{1111}+(m-1) p_{1111}\right)\right. \\
& +6(n-1)(n-2)\left(q_{211}+(m-1) p_{211}+(m-1) p_{121}\right) \\
& +3(n-1)\left(q_{22}+(m-1) p_{22}+(m-1) p_{22 \text { mix }}\right) \\
& \left.+4(n-1)\left(q_{31}+(m-1) p_{31}\right)+q_{4}+(m-1) p_{4}\right] .
\end{aligned}
$$

The notation for the four-spin overlaps is analoguous to the two-spin overlaps $q_{4}=\left\langle\sigma_{\alpha}^{\mu} \sigma_{\alpha}^{\mu} \sigma_{\alpha}^{\mu} \sigma_{\alpha}^{\mu}\right\rangle, p_{4}=\left\langle\sigma_{\alpha}^{\mu} \sigma_{\alpha}^{\mu} \sigma_{\alpha}^{\nu} \sigma_{\alpha}^{\nu}\right\rangle, q_{31}=\left\langle\sigma_{\alpha}^{\mu} \sigma_{\alpha}^{\mu} \sigma_{\alpha}^{\mu} \sigma_{\beta}^{\mu}\right\rangle, p_{31}=\left\langle\sigma_{\alpha}^{\mu} \sigma_{\alpha}^{\mu} \sigma_{\alpha}^{\nu} \sigma_{\beta}^{\nu}\right\rangle$, $q_{22}=\left\langle\sigma_{\alpha}^{\mu} \sigma_{\alpha}^{\mu} \sigma_{\beta}^{\mu} \sigma_{\beta}^{\mu}\right\rangle, p_{22}=\left\langle\sigma_{\alpha}^{\mu} \sigma_{\alpha}^{\mu} \sigma_{\beta}^{\nu} \sigma_{\beta}^{\nu}\right\rangle, p_{22 \mathrm{mix}}=\left\langle\sigma_{\alpha}^{\mu} \sigma_{\alpha}^{\nu} \sigma_{\beta}^{\nu} \sigma_{\beta}^{\mu}\right\rangle, q_{211}=\left\langle\sigma_{\alpha}^{\mu} \sigma_{\alpha}^{\mu} \sigma_{\beta}^{\mu} \sigma_{\gamma}^{\mu}\right\rangle$, $p_{211}=\left\langle\sigma_{\alpha}^{\mu} \sigma_{\alpha}^{\mu} \sigma_{\beta}^{\nu} \sigma_{\gamma}^{\nu}\right\rangle, p_{121}=\left\langle\sigma_{\alpha}^{\mu} \sigma_{\alpha}^{\nu} \sigma_{\beta}^{\nu} \sigma_{\gamma}^{\mu}\right\rangle, q_{1111}=\left\langle\sigma_{\alpha}^{\mu} \sigma_{\beta}^{\mu} \sigma_{\gamma}^{\mu} \sigma_{\delta}^{\mu}\right\rangle$ and $p_{1111}=$ $\left\langle\sigma_{\alpha}^{\mu} \sigma_{\beta}^{\mu} \sigma_{\gamma}^{\nu} \sigma_{\delta}^{\nu}\right\rangle$ (where $\alpha, \beta, \gamma, \delta$, resp. $\mu$ and $\nu$ ought to be different).

For the matrix $M_{0}$ in equation (4.13) (defined in the derivation of equation (B.16) ) we have the entries $M_{0 \alpha \beta}^{\mu \nu}=\left(x \delta_{\alpha \beta}-\lambda^{2} q_{11}^{(0)}\right) \delta_{\mu \nu}$, with $x=2(\epsilon-$ $z)-\lambda^{2} q_{2}^{(0)}+\lambda^{2} q_{11}^{(0)}$, and we find the eigenvalues $x$ (with degeneracy $m(n-1)$ ) and $x-n \lambda^{2} q_{11}^{(0)}$ (degeneracy $m$ ) leading to $\operatorname{det} M=x^{m(n-1)}\left(x-n \lambda^{2} q_{11}^{(0)}\right)^{m}$. We solve the saddle point equation for $z$ resp. $x(\overline{B .19})$, and take the limit 
$n \rightarrow 0$

$$
\begin{aligned}
0 & =x^{2}-x\left(n \lambda^{2} q_{11}^{(0)}+1\right)+\lambda^{2} q_{11}^{(0)}(n-1) \\
& \Rightarrow x_{0}=\frac{1}{2} \pm \sqrt{\frac{1}{4}+\lambda^{2} q_{11}^{(0)}}
\end{aligned}
$$

of which we choose the positive sign of the square root, since $x_{0}$ is an eigenvalue of the positive definite matrix $M_{0}$. For $\ln T_{0}$ this leads to (only regarding terms up to $\mathcal{O}(n)$, which corresponds to the replica limit $n \rightarrow 0$ in equation (4.11)

$$
\begin{aligned}
\ln T_{0}^{R S} & \propto m n\left(\epsilon-z_{0}-\frac{1}{2 n} \operatorname{Tr} \ln \left(M_{0}\right)\right) \\
& =\frac{m n}{2}\left(x_{0}+\lambda^{2} q_{2}^{(0)}-\lambda^{2} q_{11}^{(0)}\right)-\frac{m n}{2}\left(\ln \left(x_{0}\right)-\frac{\lambda^{2} q_{11}^{(0)}}{x_{0}}\right) .
\end{aligned}
$$

The overlap $q_{2}^{(0)}=\frac{2}{m n \lambda^{2}} \frac{d}{d q_{2}^{(0)}} \ln T^{R S}$ is obviously 1 and, since there is no further dependence on $q_{2}^{(0)}$ in any of the following orders of $\ln T^{R S}$, has no correction terms of any higher order. This result will hold for the broken replica symmetry case. For $q_{11}^{(00)}$ we have to calculate $\frac{\lambda^{2}}{2} m n(n-1) q_{11}^{(00)}=\left.\frac{d}{d q_{11}^{(0)}} \ln T^{R S}\right|_{q_{11}^{(0)}=q_{11}^{(00)}}$ (which means to take the derivation of $\ln T^{R S}$ with respect to $q_{11}^{(0)}$ at the value of $\left.q_{11}^{(0)}=q_{11}^{(00)}\right)$, which yields

$$
-\frac{\lambda^{2}}{2} q_{11}^{(00)}=\frac{1}{2}\left(-\lambda^{2}+\frac{\lambda^{2}}{x_{0}}+\frac{d x_{0}}{d q_{11}^{(0)}}\left(1-\frac{\lambda^{2} q_{11}^{(00)}}{x_{0}^{2}}-\frac{1}{x_{0}}\right)\right) .
$$

We find the saddle point equation (4.16) for $x_{0}$ (before taking the replica limit $n \rightarrow 0$ ) in the last bracket. This leads to

$$
\begin{aligned}
-q_{11}^{(00)} & =\frac{-\lambda^{2} q_{11}^{(00)}}{\left(\frac{1}{2}+\sqrt{\frac{1}{4}+\lambda^{2} q_{11}^{(00)}}\right)^{2}} \\
\Rightarrow q_{11}^{(00)} & =1-\frac{1}{\lambda},
\end{aligned}
$$

from which follows $x_{0}=\lambda$, i.e. we explicitly find the solution in zeroth order.

From above calculations (leading to equation (4.12) it is clear that the overlaps have no dependence on $n$. With these results we are able to calculate the four-spin overlaps $q_{\alpha \beta \gamma \delta}^{\mu \nu \eta \rho}$ using Wick's theorem [53], which allows to express them in terms of the two-spin overlaps just found. As Wick's theorem only holds for Gaussian random variables, it is by no means obvious 
that we can apply it here. However, in appendix B.5 we show that it holds for $m \rightarrow \infty$. The single component (four-spin) overlaps are

$$
\begin{aligned}
q_{1111}^{(0)} & =3\left(q_{11}^{(0)}\right)^{2} \Rightarrow q_{1111}^{(00)}=3\left(1-\frac{2}{\lambda}+\frac{1}{\lambda^{2}}\right) \\
q_{211}^{(0)} & =q_{2}^{(0)} q_{11}^{(0)}+2\left(q_{11}^{(0)}\right)^{2} \Rightarrow q_{211}^{(00)}=3-\frac{5}{\lambda}+\frac{2}{\lambda^{2}} \\
q_{22}^{(0)} & =\left(q_{2}^{(0)}\right)^{2}+2\left(q_{11}^{(0)}\right)^{2} \Rightarrow q_{22}^{(00)}=3-\frac{4}{\lambda}+\frac{2}{\lambda^{2}} \\
q_{31}^{(0)} & =3 q_{2}^{(0)} q_{11}^{(0)} \Rightarrow q_{31}^{(00)}=3\left(1-\frac{1}{\lambda}\right) \\
q_{4}^{(0)} & =3\left(q_{2}^{(0)}\right)^{2} \Rightarrow q_{4}^{(00)}=3 .
\end{aligned}
$$

The right hand side of these equations is the zeroth order result.

Higher orders are evaluated by using an appropriate Taylor expansion around $q_{11}^{(00)}$. From now on, we have to use $\operatorname{Tr}\left(M_{0}^{-1}\right)^{t}$ in various powers $t=1, \ldots, 8$. To do so, we have calculated $\left(M_{0}^{-1}\right)_{\alpha \beta}^{\mu \nu}=\left(c \delta_{\alpha \beta}+d\right) \delta_{\mu \nu}$ (i.e. with $c=\frac{1}{x_{0}}$ or $d=\frac{\lambda^{2} q_{11}}{x_{0}^{2}}$ on the diagonal of the $n$ diagonal blocks of size $m \times m$, resp. on the $n(n-1)$ non-diagonal blocks) by diagonalising $M_{0}$ and evaluated the necessary powers (Diagonalisation and matrices in appendix B.6). It should be emphasized that we only have to take the leading (zeroth) order in $n$ into account, as the overlap must not depend on $n$ (the replica limit is implied in taking only the leading order in $n$ in the derivation of the free energy in terms of $q_{\alpha \beta}^{\mu \nu}$, equation 4.12 ).

For $q_{11}^{(01)}$, we have to calculate the right hand side of $\frac{\lambda^{2}}{2} m n(n-1) q_{11}^{(0)}=$ $\frac{d}{d q_{11}^{(0)}} \ln T^{R S}$ in first order in $\frac{1}{m}$. The first order term in $\frac{1}{m}$ from equation 4.13 is $-\frac{1}{2 n m} \frac{d}{d q_{11}^{(0)}} \ln \operatorname{Tr}\left(M_{0}^{-1}\right)^{2}=-\frac{1}{2 n m} \frac{d}{d q_{11}^{(0)}} \ln T_{1}^{R S}$. We insert the zeroth order result for $q_{11}^{(0)}$ to keep it of that order and denote this proceeding by $\mathcal{O}_{0}\left(\frac{d}{d q_{11}^{(0)}} \ln T_{1}^{R S}\right)=$ $\left.\left(\frac{d}{d q_{11}^{(0)}} \ln T_{1}^{R S}\right)\right|_{q_{11}^{(0)}=q_{11}^{(00)}}$. Furthermore, we Taylor expand the first term in equation 4.13, $\frac{d}{d q_{11}^{(0)}} \ln T_{0}^{R S}$, into first order around $q_{11}^{(00)}$ and denote this order with $\mathcal{O}_{1}\left(\frac{d}{d q_{11}^{(0)}} \ln T_{0}^{R S}\right)=\left.\frac{d}{d q_{11}^{(0)}}\left(\frac{d}{d q_{11}^{(0)}} \ln T_{0}^{R S}\right)\right|_{q_{11}^{(0)}=q_{11}^{(00)}} \cdot q_{11}^{(01)}$ (which is of the order $\frac{1}{m}$ then). We note that this term includes first order overlaps $q_{11}^{(01)}$. In summary, we get the following determining equation for $q_{11}^{(01)}$

$$
-\frac{\lambda^{2}}{2} q_{11}^{(01)}=\mathcal{O}_{1}\left(\frac{d}{d q_{11}^{(0)}} \ln T_{0}^{R S}\right)-\frac{1}{2} \mathcal{O}_{0}\left(\frac{d}{d q_{11}^{(0)}} \ln T_{1}^{R S}\right)
$$


with the result $q_{11}^{(01)}=-\frac{1}{\lambda(2 \lambda-1)}$. In order to compare the replica symmetric results with the 1 -RSB results it is necessary to evaluate $x_{0}\left(q_{11}^{(0)}\right)$ up to fourth order in $\frac{1}{m}$. In first order we get $x_{01}=-\frac{\lambda}{(2 \lambda-1)^{2}}$.

Calculating higher orders of $q_{11}^{(0)}$ is straightforward. By taking the corresponding orders in $\frac{1}{m}$ in the Taylor expansion of $T^{R S}$ around $q_{11}^{(00)}$ we find the following determining equations

$$
\begin{aligned}
-\frac{\lambda^{2}}{2} q_{11}^{(02)}= & \mathcal{O}_{2}\left(\frac{d}{d q_{11}^{(0)}} \ln T_{0}^{R S}\right)-\frac{1}{2} \mathcal{O}_{1}\left(\frac{d}{d q_{11}^{(0)}} \ln T_{1}^{R S}\right)+\mathcal{O}_{0}\left(\frac{d}{d q_{11}^{(0)}} \ln T_{2}^{R S}\right) \\
-\frac{\lambda^{2}}{2} q_{11}^{(03)}= & \mathcal{O}_{3}\left(\frac{d}{d q_{11}^{(0)}} \ln T_{0}^{R S}\right)-\frac{1}{2} \mathcal{O}_{2}\left(\frac{d}{d q_{11}^{(0)}} \ln T_{1}^{R S}\right)+\mathcal{O}_{1}\left(\frac{d}{d q_{11}^{(0)}} \ln T_{2}^{R S}\right)+\mathcal{O}_{0}\left(\frac{d}{d q_{11}^{(0)}} \ln T_{3}^{R S}\right) \\
-\frac{\lambda^{2}}{2} q_{11}^{(04)}= & \mathcal{O}_{4}\left(\frac{d}{d q_{11}^{(0)}} \ln T_{0}^{R S}\right)-\frac{1}{2} \mathcal{O}_{3}\left(\frac{d}{d q_{11}^{(0)}} \ln T_{1}^{R S}\right)+\mathcal{O}_{2}\left(\frac{d}{d q_{11}^{(0)}} \ln T_{2}^{R S}\right) \\
& +\mathcal{O}_{1}\left(\frac{d}{d q_{11}^{(0)}} \ln T_{3}^{R S}\right)+\mathcal{O}_{0}\left(\frac{d}{d q_{11}^{(0)}} \ln T_{4}^{R S}\right) .
\end{aligned}
$$

For instance, the notation $\mathcal{O}_{2}\left(f\left(q_{11}^{(0)}\right)\right)$ stands for $\left.\frac{d}{d q_{11}^{(0)}}\left(f\left(q_{11}^{(0)}\right)\right)\right|_{q_{11}^{(0)}=q_{11}^{(00)}}$. $q_{11}^{(02)}+\left.\frac{1}{2} \frac{d^{2}}{d\left(q_{11}^{(0)}\right)^{2}}\left(f\left(q_{11}^{(0)}\right)\right)\right|_{q_{11}^{(0)}=q_{11}^{(00)}} \cdot\left(q_{11}^{(01)}\right)^{2}$. We see that this procedure is iterative, since one needs to know the lower order results in order to solve the higher order equations. The solutions are

$$
\begin{aligned}
q_{11}^{(02)}= & \frac{216 \lambda^{2}-136 \lambda+1}{2 \lambda(2 \lambda-1)^{4}} \\
q_{11}^{(03)}= & \frac{112872 \lambda^{4}-203352 \lambda^{3}+122884 \lambda^{2}-24946 \lambda-1}{2 \lambda(2 \lambda-1)^{7}} \\
q_{11}^{(04)}= & \frac{1}{24 \lambda(2 \lambda-1)^{10}}\left(161280 \lambda^{11}-4783680 \lambda^{10}+12096864 \lambda^{9}-7224624 \lambda^{8}\right. \\
& +296576352 \lambda^{7}-2774291654 \lambda^{6}+7537191576 \lambda^{5}-9983356072 \lambda^{4} \\
& \left.+7432229600 \lambda^{3}-3191593212 \lambda^{2}+741447184 \lambda-72398659\right)
\end{aligned}
$$

with $x_{02}=\frac{\lambda^{2} q_{11}^{02}}{2 \lambda-1}-\frac{\lambda^{2}}{(2 \lambda-1)^{5}}, x_{03}=\frac{\lambda^{2} q_{11}^{03}}{2 \lambda-1}+\frac{2 \lambda x_{02}}{(2 \lambda-1)^{3}}$ and $x_{04}=\frac{\lambda^{2} q_{11}^{04}}{2 \lambda-1}+\frac{2 \lambda x_{03}}{(2 \lambda-1)^{3}}-\frac{\left(x_{02}\right)^{2}}{2 \lambda-1}$. In the following subsection we will evaluate the corresponding results for the 1-RSB-ansatz to gain some insight into the breaking of replica symmetry for finite $m$. 


\section{1-RSB}

In the case of one step broken replica symmetry, according to the usual replica symmetry breaking scheme [8] (see also the introduction of this thesis), the $n$ replicas separate into $\frac{n}{r}$ blocks $\Omega_{K}(K=1, \ldots, n / r)$ with $r$ replicas apiece. Spins from replicas within the same block have a mutual overlap of $q_{11,2}=$ $\left\langle\sigma_{\alpha, K}^{\mu} \sigma_{\beta, K}^{\mu}\right\rangle$ and spins from replicas taken from different blocks have an overlap of $q_{11,11}=\left\langle\sigma_{\alpha, K}^{\mu} \sigma_{\beta, L}^{\mu}\right\rangle$ ( $K$ and $L$ denoting the block number). Alike to the previous section, we have to take the replica limit properly, which means to find the first order in $n$ of the right hand side of equation (4.12). Hence, the overlaps will not depend on $n$, and $r$ will finally be in between zero and one (as $1 \leq r \leq n$ before taking the replica limit). The replica symmetric ansatz of equation 4.15) changes in the case of 1 -RSB to

$$
\begin{aligned}
& \sum_{\substack{\alpha, \beta \\
\mu, \nu}} q_{\alpha \beta}^{\mu \nu}=\sum_{K, L} \sum_{\substack{\left.\alpha \in \Omega_{K} \neq \Omega_{L} \ni \beta\right) \\
\mu, \nu}} q_{11,11} \delta_{\mu \nu}+\sum_{K} \sum_{\substack{\alpha \neq \beta) \in \Omega_{K} \\
\mu, \nu}} q_{11,2} \delta_{\mu \nu}+\sum_{K} \sum_{\substack{\alpha \in \Omega_{K} \\
\mu, \nu}} q_{2,2} \delta_{\mu \nu} \\
& =m n(n-r) q_{11,11}+m n(r-1) q_{11,2}+m n q_{2,2} \\
& \sum_{\substack{\alpha, \beta, \gamma, \delta \\
\mu, \nu, \rho}} q_{\alpha \beta \gamma \delta}^{\mu \nu \eta \rho}=m n(n-r)(n-2 r)(n-3 r) q_{1111,1111}+6 m n(r-1)(n-r)(n-2 r) q_{1111,211} \\
& \quad+3 m n(n-r)(r-1)(r-1) q_{1111,22}+4 m n(n-r)(r-1)(r-2) q_{1111,31} \\
& +m n(r-1)(r-2)(r-3) q_{1111,4}+6 m n(n-r)(n-2 r) q_{211,211}+6 m n(n-r)(r-1) q_{211,22} \\
& +12 m n(r-1)(n-r) q_{211,31}+6 m n(r-1)(r-2) q_{211,4}+3 m n(n-r) q_{22,22} \\
& \quad+3 m n(r-1) q_{22,4}+4 m n(n-r) q_{31,31}+4 m n(r-1) q_{31,4}+m n q_{4,4}+\cdots .
\end{aligned}
$$

We omit those four-spin overlaps which contain different components. They do not contribute in the following, as we will see in section 4.3.3. To evaluate $\ln T_{0}^{1 R S B}$ we have to diagonalize the Matrix $M_{0}$ for this ansatz, which has the entries $M_{0}^{\mu \nu}{ }_{\alpha \in \Omega_{K} \beta \in \Omega_{L}}=\left(\left(y+\lambda^{2} q_{11,11}^{(0)}\right) \delta_{\alpha \beta}-\lambda^{2} q_{11,2}^{(0)} \delta_{K L}-\lambda^{2} q_{11,11}^{(0)}\right) \delta_{\mu \nu}$, with $y=$ $2(\epsilon-z)-\lambda^{2} q_{2,2}^{(0)}+\lambda^{2} q_{11,2}^{(0)}$. We find the eigenvalues $y$ (with degeneracy $m \frac{n}{r}(r-$ 1)), $y_{2}=y-r \lambda^{2}\left(q_{11,2}^{(0)}-q_{11,11}^{(0)}\right)\left(\right.$ degeneracy $\left.m\left(\frac{n}{r}-1\right)\right)$ and $y_{3}=y-r \lambda^{2}\left(q_{11,2}^{(0)}-\right.$ $\left.q_{11,11}^{(0)}\right)-n \lambda^{2} q_{11,11}^{(0)}($ degeneracy $m$ ) (for further details see appendix B.6). This gives $\operatorname{det} M=y^{m(r-1) n / r} y_{2}^{m\left(\frac{n}{r}-1\right)} y_{3}^{m}$, which simplifies when neglecting terms 
of order $n^{2}$ and higher, and yields for equation 4.13 in zeroth order in $\frac{1}{m}$

$$
\begin{aligned}
\ln T_{0}^{1 R S B} \propto \frac{m n}{2}\left(y_{0}+\lambda^{2} q_{2,2}^{(0)}-\right. & \left.\lambda^{2} q_{11,2}^{(0)}\right)-\frac{m n}{2}\left(\ln \left(y_{0}\right)-\frac{1}{r} \ln \left(y_{0}\right)\right. \\
+ & \left.\frac{1}{r} \ln \left(y_{0}-r \lambda^{2} \Delta q\right)-\frac{\lambda^{2} q_{11,11}^{(0)}}{y_{0}-r \lambda^{2} \Delta q}\right)
\end{aligned}
$$

where we abbreviated $q_{11,2}^{(0)}-q_{11,11}^{(0)}$ to $\Delta q$. The saddle point equation for $z$, resp. $y$ (with the solution $y_{0}$ ) is $1=\frac{1}{y_{0}}-\frac{1}{r y_{0}}+\frac{1}{r\left(y_{0}-r \lambda^{2} \Delta q\right)}+\frac{\lambda^{2} q_{11,11}^{(0)}}{\left(y_{0}-r \lambda^{2} \Delta q\right)^{2}}$, or equivalently

$$
\begin{aligned}
0= & y_{0}\left(y_{0}-r \lambda^{2}\left(q_{11,2}-q_{11,11}\right)\right)^{2}-y_{0}\left(y_{0}-r \lambda^{2}\left(q_{11,2}-q_{11,11}\right)\right) \\
& +\left(1-\frac{1}{r}\right) r \lambda^{2}\left(q_{11,2}-q_{11,11}\right)\left(y_{0}-r \lambda^{2}\left(q_{11,2}-q_{11,11}\right)\right)-y_{0} \lambda^{2} q_{11,11} .
\end{aligned}
$$

We will denote this equation by saddle $_{y}\left(q_{11,2}^{(0)}, q_{11,11}^{(0)}\right)$. Unfortunately it is not generally solvable. Nevertheless we can derive the desired results by making use of it to solve the following equations, which determine the overlaps $q_{11,2}^{(0)}$ and $q_{11,11}^{(0)}$, where

$$
\begin{aligned}
\frac{\lambda^{2}}{2} m n(r-1) q_{11,2}^{(00)} & =\left.\frac{d}{d q_{11,2}^{(0)}} \ln T_{0}^{1 R S B}\right|_{q_{11, x}^{(0)}=q_{11, x}^{(00)}} \\
\Rightarrow(r-1) q_{11,2}^{(00)} & =-1+\frac{1}{y_{00}-r \lambda^{2} \Delta q_{0}}+\frac{r \lambda^{2} q_{11,11}^{(00)}}{\left(y_{00}-r \lambda^{2} \Delta q_{0}\right)^{2}}
\end{aligned}
$$

We find the derivative $\frac{d y_{0}}{d q_{11,2}^{(0)}}$ by differentiating $\operatorname{saddl}_{y}\left(q_{11,2}^{(0)}, q_{11,11}^{(0)}\right)$ (equation (4.26) ) with respect to $q_{11,2}^{(0)}$ and get

$$
\frac{d y_{0}}{d q_{11,2}^{(0)}}=\frac{2 r \lambda^{2} y_{0}^{2}-2 r^{2} \lambda^{4} y_{0} \Delta q-2 r \lambda^{2} y_{0}+\lambda^{2} y_{0}+2 r^{2} \lambda^{4} \Delta q-2 r \lambda^{4} \Delta q}{3 y_{0}^{2}-4 r \lambda^{2} y_{0} \Delta q+r^{2} \lambda^{4}(\Delta q)^{2}-2 y_{0}+2 r \lambda^{2} \Delta q-\lambda^{2} q_{11,2}^{(0)}}
$$

As for the replica symmetric calculation, the notation $\mathcal{O}_{0}\left(\frac{d y_{0}}{d q_{11,2}^{(0)}}\right)$ means inserting $q_{11,2}^{(00)}$ and $q_{11,11}^{(00)}$ for the general overlaps $q_{11,2}^{(0)}$ and $q_{11,11}^{(0)}$ into equation 4.28. The notations $y_{00}$ and $\Delta q_{0}$ denote the zeroth order results in $\frac{1}{m}$ for 
$y_{0}$ and $\Delta q$ resp.. Analogously to equation (4.27) we derive

$$
\begin{gathered}
\frac{\lambda^{2}}{2} m n(n-r) q_{11,11}^{(00)}=\left.\frac{d}{d q_{11,11}^{(0)}} \ln T_{0}^{1 R S B}\right|_{q_{11, x}^{(0)}=q_{11, x}^{(00)}} \\
\Rightarrow(n-r) q_{11,11}^{(00)}=\frac{-r \lambda^{2} q_{11,11}^{(00)}}{\left(y_{00}-r \lambda^{2} \Delta q_{0}\right)^{2}}
\end{gathered}
$$

The last line of this equation contains the useful result (for $n \rightarrow 0$ and $q_{11.11}^{(00)} \neq$ 0) $y_{00}-r \lambda^{2} \Delta q_{0}=\lambda$. With it we rewrite equation 4.27) to $(r-1) q_{11,2}^{(00)}=$ $-1+\frac{1}{\lambda}+r q_{11,11}^{(00)}$. Inserting both results into the saddle point equation 4.26 leads to

$$
\begin{aligned}
r \lambda \Delta q_{0}\left(1-q_{11,11}^{(00)}\right) & =q_{11,2}^{(00)}-1+\frac{1}{\lambda} \\
& \Rightarrow q_{11,11}^{(00)}=1-\frac{1}{\lambda}
\end{aligned}
$$

which follows after again using $(r-1) q_{11,2}^{(00)}=-1+\frac{1}{\lambda}+r q_{11,11}^{(00)}$ and for $\Delta q_{0} \neq 0$. However, this leads immediately to $q_{11,2}^{(00)}=1-\frac{1}{\lambda}$ causing a contradiction to $\Delta q_{0} \neq 0$. Therefore replica symmetry holds for $m \rightarrow \infty$, i.e. we have reproduced this important result from Ref. [41].

To see whether replica symmetry is broken for finite $m$ with the one step replica symmetry breaking ansatz, we have to evaluate higher orders of $q_{11,2}^{(0)}$ and $q_{11,11}^{(0)}$. In principle, we follow the replica symmetric procedure of the previous subsection, but we have to be aware of the missing explicit solution for $y_{0}\left(q_{11,2}^{(0)}, q_{11,11}^{(0)}\right)$. That is why we treat $y_{0}$ (and for convenience $y_{2}=y_{0}-r \lambda^{2} \Delta q$ ) as further variables of $T^{1 R S B}\left(y_{0}\left(q_{11,2}^{(0)}, q_{11,11}^{(0)}\right), y_{2}\left(q_{11,2}^{(0)}, q_{11,11}^{(0)}\right), q_{11,2}^{(0)}, q_{11,11}^{(0)}\right)$. We get a third equation for $y_{0 x}$ additionally to the determining equations for $q_{11,2}^{(0 x)}$ and $q_{11,11}^{(0 x)}$ by expanding saddle ${ }_{y}\left(q_{11,2}^{(0)}, q_{11,11}^{(0)}\right)$ to the corresponding order $x$. Furthermore, we have to take account of the derivatives $\frac{d y_{0}}{d q_{11,2}^{(0)}}$ and $\frac{d y_{0}}{d q_{11,11}^{(0)}}$, which occur, for instance, in $\frac{d}{d q_{11,2}^{(0)}} \ln T^{1 R S B}$. They have to be expanded to the necessary higher orders as well.

As in the replica symmetric case, we have to calculate $\operatorname{Tr}\left(M_{0}^{-1}\right)^{t}$ up to $t=8$. We give the result for $n=0$ here and refer to the appendix B.6 for further details on powers of $\operatorname{Tr}\left(M_{0}^{-1}\right)$ :

$$
\left(M_{0}^{-1}\right)_{\alpha \in \Omega_{K}, \beta \in \Omega_{L}}^{\mu \nu}=\left(c_{1} \delta_{\alpha \beta}+c_{2} \delta_{K L}+c_{3}\right) \delta_{\mu \nu},
$$

where $c_{1}=\frac{1}{y}, c_{2}=-\frac{1}{y_{r}}+\frac{1}{y_{2} r}$ and $c_{3}=-\frac{1}{y_{2} n}+\frac{1}{y_{3} n}$. We find the equations determining the orders $\frac{1}{m}$ to $\frac{1}{m^{4}}$ analoguously to the replica symmetric 
case in equations (4.21) and 4.22) by regarding the corresponding orders of $\frac{d}{d q_{11,2}^{(0)}} \ln T^{1 R S B}$ and $\frac{d}{d q_{11,11}^{(0)}} \ln T^{1 R S B}$. Additionally, we have to expand the saddle point equation for $y$, saddle $e_{y}\left(q_{11,2}^{(0)}, q_{11,11}^{(0)}\right)$.

For all orders $x$ the system of equations for $y_{0 x}, q_{11,2}^{(0 x)}$ and $q_{11,11}^{(0 x)}$ is (with a different right hand side $\left.\vec{L}_{x}(r)\right)$

$$
\left(\begin{array}{ccc}
1-2 \lambda & 1+2 r(\lambda-1) & -2 r(\lambda-1) \\
-1-2 r(\lambda-1) & 1+2 r^{2}(\lambda-1) & -2 r^{2}(\lambda-1) \\
2 r(\lambda-1) & -2 r^{2}(\lambda-1) & 2 r^{2}(\lambda-1)
\end{array}\right)\left(\begin{array}{c}
y_{0 x} \\
\lambda^{2} q_{11,2}^{(0 x)} \\
\lambda^{2} q_{11,11}^{(0 x)}
\end{array}\right)=\vec{L}_{x}(r)
$$

since the matrix on the left hand side is solely determined by the first order derivative of $\operatorname{saddle}_{y}\left(q_{11,2}^{(0)}, q_{11,11}^{(0)}\right), \frac{d}{d q_{11,2}^{(0)}} \ln T_{0}^{1 R S B}$ and $\frac{d}{d q_{11,11}^{(0)}} \ln T_{0}^{1 R S B}$ resp. and therefore appears to be the same for each order $x$. The three equations are not linearly independent but have in general a two dimensional solution space. To see whether this system of equations is solvable, we use the left eigenvector to the eigenvalue zero, which is $\vec{l}=\left(-1,1,1-\frac{1}{r}\right)$, and multiply it to $\vec{L}_{x}(r)$, which must yield zero in order to make the solution exist. In first order we find

$$
\vec{L}_{1}(r)=\left(\begin{array}{c}
0 \\
-2(r-1)(\lambda-1) x_{01} \\
2 r(\lambda-1) x_{01}
\end{array}\right)
$$

which satisfies $\vec{l} \cdot \vec{L}_{1}(r)=0$. Due to the similar structure of equation 4.32 in each order in $\frac{1}{m}$, we find two solutions (in each order) of the general form $\vec{u}_{1}=\left(0, \lambda^{2} q_{a}, \lambda^{2} q_{b}\right)$ and $\vec{u}_{2}=\left(a, \lambda^{2} q, \lambda^{2} q\right)$ (the replica symmetric solution), with $\lambda^{2} q_{a}=L_{x 2}(r)+L_{x 3}(r), \lambda^{2} q_{b}=\lambda^{2} q_{a}+\frac{L_{x 3}(r)}{2 r^{2}(\lambda-1)}, q=q_{11}^{(0 x)}$ and $a=x_{0 x}$ (with $L_{x y}$ we mean the component $y$ of the solution vector in order $x$ ). The solution in first order $\vec{u}_{01}=\vec{u}_{1}+\alpha_{1}(r)\left(\vec{u}_{2}-\vec{u}_{1}\right.$ ) (with a parameter $\alpha_{1}$ scanning the solution space) then is

$$
\begin{aligned}
\vec{u}_{01} & =\left(\begin{array}{c}
y_{01} \\
\lambda^{2} q_{11,2}^{(01)} \\
\lambda^{2} q_{11,11}^{(01)}
\end{array}\right) \\
& =\left(\begin{array}{c}
0 \\
\frac{2 \lambda(1-\lambda)}{(2 \lambda-1)^{2}} \\
-\lambda \frac{1+2 r(\lambda-1)}{r(2 \lambda-1)^{2}}
\end{array}\right)-\frac{\lambda}{(2 \lambda-1)^{2}} \alpha_{1}(r) \cdot\left(\begin{array}{c}
1 \\
1 \\
1-\frac{1}{r}
\end{array}\right) .
\end{aligned}
$$

We see that the solution space for 1-RSB includes the replica symmetric solution. In principle, we could insert this solution space into the free energy, 
equation (5.24), and maximize it with respect to $r$ and $\alpha_{1}(r)$. Despite not knowing the special dependence of $\alpha_{1}$ on $r$, the free energy is independent of both parameters, see appendix B.7. A different, successful way to get the final value of $\alpha_{1}$ is to calculate $L_{2}(r)$, i.e. the solution vector of the system of equations in second order, following the presented expansion method. This includes squares of the solutions in first order, $y_{01}, q_{11,2}^{(01)}$ and $q_{11,11}^{(01)}$, due to the expansion of the Taylor series up to second order. If there is a solution in second order, the product $\vec{l} \cdot \vec{L}_{2}(r)$ must be zero and indeed we find $\alpha_{1}(r)^{2}-$ $2 \alpha_{1}(r)=1 \Rightarrow \alpha_{1}=1$. This yields a replica symmetric result in first order with the corresponding values from the replica symmetric evaluation from section 4.3.2 $q_{11,2}^{(01)}=q_{11,11}^{(01)}=q_{11}^{(01)}$ and $y_{01}=x_{01}$. The vector $L_{2}(r)$ (with $\left.\alpha_{1}=1\right)$ is

$$
L_{2}(r)=\left(\begin{array}{c}
\frac{1}{(2 \lambda-1)^{4}} \\
-2(r-1)(\lambda-1) x_{02}+\frac{1}{(2 \lambda-1)^{4}} \\
2 r(\lambda-1) x_{02}
\end{array}\right)
$$

The solution in second order can again be constructed by calculating the explicit form of the general solution vectors $\vec{u}_{1}$ and $\vec{u}_{2}$, and build $\vec{u}_{02}=$ $\vec{u}_{1}+\alpha_{2}(r)\left(\vec{u}_{2}-\vec{u}_{1}\right)$ (with a new parameter $\alpha_{2}(r)$ spanning the solution space). We get

$$
\begin{aligned}
\vec{u}_{02} & =\left(\begin{array}{c}
y_{02} \\
\lambda^{2} q_{11,2}^{(02)} \\
\lambda^{2} q_{11,11}^{(02)}
\end{array}\right) \\
& =\left(\begin{array}{c}
2(\lambda-1) x_{02}+\frac{1}{(2 \lambda-1)^{4}} \\
2(\lambda-1) x_{02}+\frac{1}{(2 \lambda-1)^{4}}+\frac{x_{02}}{r}
\end{array}\right)+\alpha_{2}(r) x_{02} \cdot\left(\begin{array}{c}
1 \\
1 \\
1-\frac{1}{r}
\end{array}\right) .
\end{aligned}
$$

The free energy is again independent of the choice of $\alpha_{2}(r)$ and $r$.

The iterative character of this procedure forces us to go to even higher orders, in order to find the value of $\alpha_{2}(r)$. The resulting solution vector in third order is independent of $\alpha_{2}(r)$

$$
L_{3}(r)=\left(\begin{array}{c}
-\frac{2 \lambda x_{02}}{(2 \lambda-1)^{2}} \\
-2(r-1)(\lambda-1) x_{03}-\frac{2 \lambda x_{02}}{(2 \lambda-1)^{2}} \\
2 r(\lambda-1) x_{03}
\end{array}\right)
$$


and the overlaps are

$$
\begin{aligned}
& \vec{u}_{03}=\left(\begin{array}{c}
y_{03} \\
\lambda^{2} q_{11,2}^{(03)} \\
\lambda^{2} q_{11,11}^{(03)}
\end{array}\right) \\
= & \left(\begin{array}{c}
0 \\
L_{32}(r)+L_{33}(r) \\
L_{32}(r)+L_{33}(r)+\frac{L_{33}(r)}{2 r^{2}(\lambda-1)}
\end{array}\right)+\alpha_{3}(r) \cdot\left(\begin{array}{c}
\frac{L_{33}(r)}{2 r(\lambda-1)} \\
\frac{L_{33}(r)}{2 r(\lambda-1)}+L_{31}(r)-L_{32}(r)-L_{33}(r) \\
\frac{L_{33}(r)}{2 r(\lambda-1)}+L_{31}(r)-L_{32}(r)-L_{33}(r)-\frac{L_{33}(r)}{2 r^{2}(\lambda-1)}
\end{array}\right) .
\end{aligned}
$$

The next order yields a solution for $\alpha_{2}(r)$. It is

$$
L_{4}(r)=\left(\begin{array}{c}
\left((r-1)\left(2 \alpha_{2}(r)-\left(\alpha_{2}(r)\right)^{2}\right)-1\right) \frac{\left(x_{02}\right)^{2}}{r \lambda}-\frac{\left(x_{02}\right)^{2}}{r \lambda}-(2 \lambda-1) x_{04}+\lambda^{2} q_{11}^{(04)} \\
-2(r-1)(\lambda-1) x_{04}-(2 \lambda-1) x_{04}+\lambda^{2} q_{11}^{(04)} \\
2 r(\lambda-1) x_{04}
\end{array}\right)
$$

and forces $\alpha_{2}(r)$ to be equal to one, in order to satisfy $\vec{l} \cdot \vec{L}_{4}(r)=0$. This result corresponds to the replica symmetric solution found in the previous section. Therefore the $m$-component spin glass favours replica symmetry up to order $\frac{1}{m^{2}}$ instead of breaking it in a first step of replica symmetry breaking. This result does not reproduce the findings of Ref. [41], where replica symmetry is broken for all finite $m$. However, in contrast to us their method of choice is the calculation of the eigenvalues of the Hessian (the matrix which governs the fluctuations around the replica symmetric free energy). In order $\frac{1}{m^{2}}$ some of these eigenvalues become negative.

\subsubsection{Corrections in $\frac{1}{k}$}

In order to calculate the $\frac{1}{k}$-corrections to the overlaps $q$ from the previous section, we have to take along the $\frac{1}{k}$-terms in the expression for $g_{n}^{k}$ (equation (4.10)). First of all, we use bimodal $J_{i j}$, i.e. bonds distributed according to the $\pm J$ model, and analyse the case of Gaussian bonds afterwards. We want to calculate $q_{i j}^{s t}=\frac{\operatorname{Tr}_{\sigma} \sigma_{i}^{s} \sigma_{j}^{t} g_{n}^{k}(\vec{\sigma})}{\operatorname{Tr}_{\sigma} g_{n}^{k}(\vec{\sigma})}$ to order $\frac{1}{k}$. To this end, we expand this equation by a factor $\operatorname{Tr}_{\sigma} \exp \left(\frac{\lambda^{2}}{2} \sum_{\substack{\alpha, \beta \\ \mu, \nu}}^{\mu \nu(0)} \sigma_{\alpha}^{\mu} \sigma_{\beta}^{\nu}\right)$ and get $q_{i j}^{s t}=\frac{\operatorname{Tr}_{\sigma} \sigma_{i}^{s} \sigma_{j}^{t} g_{n}^{k}(\vec{\sigma})}{\operatorname{Tr}_{\sigma} \exp \left(\frac{\lambda^{2}}{2} \sum_{\substack{\alpha, \beta \\ \mu, \nu}}^{\mu \nu(0)} \sigma_{\alpha}^{\mu} \sigma_{\beta}^{\nu}\right)}\left(\frac{\operatorname{Tr}_{\sigma} g_{n}^{k}(\vec{\sigma})}{\operatorname{Tr}_{\sigma} \exp \left(\frac{\lambda^{2}}{2} \sum_{\substack{\alpha, \beta \\ \mu, \nu}}^{\mu \nu(0)} q_{\alpha \beta}^{\mu} \sigma_{\beta}^{\nu}\right)}\right)^{-1}$. To order $\frac{1}{k}$ this 
is

$$
\begin{gathered}
q_{i j}^{s t(1)}=\operatorname{Tr}_{\sigma} \sigma_{i}^{s} \sigma_{j}^{t} \exp \left(\frac{\lambda^{2}}{2} \sum_{\substack{\alpha, \beta \\
\mu, \nu}} q_{\alpha \beta}^{\mu \nu(0)} \sigma_{\alpha}^{\mu} \sigma_{\beta}^{\nu}\right)\left(\frac{\lambda^{2}}{2} \sum_{\substack{\alpha, \beta \\
\mu, \nu}} q_{\alpha \beta}^{\mu \nu(1)} \sigma_{\alpha}^{\mu} \sigma_{\beta}^{\nu}\right. \\
\left.+\frac{\lambda^{4}}{24} \sum_{\substack{\alpha, \beta, \gamma, \delta \\
\mu, \nu, \eta, \rho}} q_{\alpha \beta \gamma \delta}^{\mu \nu \eta \rho(0)} \sigma_{\alpha}^{\mu} \sigma_{\beta}^{\nu} \sigma_{\gamma}^{\eta} \sigma_{\delta}^{\rho}-\frac{\lambda^{4}}{8}\left(\sum_{\substack{\alpha, \beta \\
\mu, \nu}}^{\mu \nu(0)} q_{\alpha \beta}^{\mu} \sigma_{\beta}^{\nu}\right)^{2}\right) /\left(\operatorname{Tr}_{\sigma} \exp \left(\frac{\lambda^{2}}{2} \sum_{\substack{\alpha, \beta \\
\mu, \nu}} q_{\alpha \beta}^{\mu \nu(0)} \sigma_{\alpha}^{\mu} \sigma_{\beta}^{\nu}\right)\right) \\
-q_{i j}^{s t(0)} \operatorname{Tr}_{\sigma} \exp \left(\frac{\lambda^{2}}{2} \sum_{\substack{\alpha, \beta \\
\mu, \nu}} q_{\alpha \beta}^{\mu \nu(0)} \sigma_{\alpha}^{\mu} \sigma_{\beta}^{\nu}\right)\left(\frac{\lambda^{2}}{2} \sum_{\substack{\alpha, \beta \\
\mu, \nu}} q_{\alpha \beta}^{\mu \nu(1)} \sigma_{\alpha}^{\mu} \sigma_{\beta}^{\nu}\right. \\
\left.+\frac{\lambda^{4}}{24} \sum_{\substack{\alpha, \beta, \gamma, \delta \\
\mu, \nu, \eta, \rho}} q_{\alpha \beta \gamma \delta}^{\mu \nu \eta \rho(0)} \sigma_{\alpha}^{\mu} \sigma_{\beta}^{\nu} \sigma_{\gamma}^{\eta} \sigma_{\delta}^{\rho}-\frac{\lambda^{4}}{8}\left(\sum_{\substack{\alpha, \beta \\
\mu, \nu}} q_{\alpha \beta}^{\mu \nu(0)} \sigma_{\alpha}^{\mu} \sigma_{\beta}^{\nu}\right)^{2}\right) /\left(\operatorname{Tr}_{\sigma} \exp \left(\frac{\lambda^{2}}{2} \sum_{\substack{\alpha, \beta \\
\mu, \nu}} q_{\alpha \beta}^{\mu \nu(0)} \sigma_{\alpha}^{\mu} \sigma_{\beta}^{\nu}\right)\right) .
\end{gathered}
$$

We can simplify the parts including four-spin overlaps in this equation by using Wick's theorem to write (regarding that each component has to occur at least twice in the four-spin overlaps)

$$
\begin{gathered}
\left(\frac{\lambda^{4}}{24 k} \sum_{\substack{\alpha, \beta, \gamma, \delta \\
\mu, \nu, \eta, \rho}} q_{\alpha \beta \gamma \delta}^{\mu \nu \eta \rho(0)}\left(\delta_{\mu \nu} \delta_{\eta \rho}+\delta_{\mu \eta} \delta_{\nu \rho}+\delta_{\mu \rho} \delta_{\eta \nu}\right)-\frac{\lambda^{4}}{8 k} \sum_{\substack{\alpha, \beta, \gamma, \delta \\
\mu, \nu, \eta, \rho}} q_{\alpha \beta}^{\mu \nu(0)} q_{\gamma \delta}^{\eta \rho(0)} \delta_{\mu \nu} \delta_{\eta \rho}\right) \sigma_{\alpha}^{\mu} \sigma_{\beta}^{\nu} \sigma_{\gamma}^{\eta} \sigma_{\delta}^{\rho} \\
=\left(\frac{\lambda^{4}}{24 k} \sum_{\substack{\alpha, \beta, \gamma, \delta \\
\mu, \nu, \eta, \rho}}\left(q_{\alpha \beta}^{\mu \nu(0)} q_{\gamma \delta}^{\eta \rho(0)}+q_{\alpha \gamma}^{\mu \eta(0)} q_{\beta \delta}^{\nu \rho(0)}+q_{\alpha \delta}^{\mu \rho(0)} q_{\beta \gamma}^{\nu \eta(0)}\right)\left(\delta_{\mu \nu} \delta_{\eta \rho}+\delta_{\mu \eta} \delta_{\nu \rho}+\delta_{\mu \rho} \delta_{\eta \nu}\right)\right. \\
\left.-\frac{\lambda^{4}}{8 k} \sum_{\substack{\alpha, \beta, \gamma, \delta \\
\mu, \nu, \eta, \rho}} q_{\alpha \beta}^{\mu \nu(0)} q_{\gamma \delta}^{\eta \rho(0)} \delta_{\mu \nu} \delta_{\eta \rho}\right) \sigma_{\alpha}^{\mu} \sigma_{\beta}^{\nu} \sigma_{\gamma}^{\eta} \sigma_{\delta}^{\rho}=0 .
\end{gathered}
$$

We have regarded that $q_{\alpha \beta}^{\mu \nu}$ is only non-zero for $\mu=\nu$ and switched the indices of the remaining three terms of the first sum (e.g. $\sum_{\substack{\alpha, \beta, \gamma, \gamma, \rho \\ \mu, \nu}}^{\mu \eta(0)} q_{\beta \delta}^{\nu \rho(0)} \sigma_{\alpha}^{\mu} \sigma_{\beta}^{\nu} \sigma_{\gamma}^{\eta} \sigma_{\delta}^{\rho} \delta_{\mu \eta} \delta_{\nu \rho} \rightarrow$ $\left.\sum_{\substack{\alpha, \beta, \gamma, \delta \\ \mu, \nu, \rho}}^{\mu \nu(0)} q_{\alpha \delta}^{\eta \rho(0)} \sigma_{\alpha}^{\mu} \sigma_{\beta}^{\nu} \sigma_{\gamma}^{\eta} \sigma_{\delta}^{\rho} \delta_{\mu \nu} \delta_{\eta \rho}\right)$ in the last step in such a way as to cancel them with the second sum. We note that these terms would not have cancelled if we had used Gaussian distributed $J_{i j}$ (due to the different prefactor 
of the first sum, $\frac{1}{24} \rightarrow \frac{1}{8}$ ). With equation 4.41 we get

$$
\begin{aligned}
q_{i j}^{s t(1)} & =\frac{\operatorname{Tr}_{\sigma} \sigma_{i}^{s} \sigma_{j}^{t} \exp \left(\frac{\lambda^{2}}{2} \sum_{\mu, \nu}^{\alpha, \beta} q_{\alpha \beta}^{\mu \nu(0)} \sigma_{\alpha}^{\mu} \sigma_{\beta}^{\nu}\right)\left(\frac{\lambda^{2}}{2} \sum_{\substack{\alpha, \beta \\
\mu, \nu}} q_{\alpha \beta}^{\mu \nu(1)} \sigma_{\alpha}^{\mu} \sigma_{\beta}^{\nu}\right)}{\operatorname{Tr}_{\sigma} \exp \left(\frac{\lambda^{2}}{2} \sum_{\substack{\alpha, \beta \\
\mu, \nu}}^{\mu \nu(0)} \sigma_{\alpha}^{\mu} \sigma_{\beta}^{\nu}\right)} \\
- & q_{i j}^{s t(0)} \frac{\operatorname{Tr}_{\sigma} \exp \left(\frac{\lambda^{2}}{2} \sum_{\substack{\alpha, \beta \\
\mu, \nu}}^{\mu \nu(0)} q_{\alpha}^{\mu} \sigma_{\beta}^{\nu}\right)\left(\frac{\lambda^{2}}{2} \sum_{\substack{\alpha, \beta \\
\alpha, \nu}} q_{\alpha \beta}^{\mu \nu(1)} \sigma_{\alpha}^{\mu} \sigma_{\beta}^{\nu}\right)}{\operatorname{Tr}_{\sigma} \exp \left(\frac{\lambda^{2}}{2} \sum_{\substack{\alpha, \beta \\
\mu, \nu}}^{\mu \nu(0)} \sigma_{\alpha}^{\mu} \sigma_{\beta}^{\nu}\right)} .
\end{aligned}
$$

From now on it is a combinatorical exercise to derive the desired results. As already mentioned there are only overlaps for $s=t$. We point out that possible four-spin overlaps consisting of two pairs of explicitly different components do not contribute to $q_{i j}^{s t(1)}$ (e.g. $p_{1111}$ or $p_{22}$ ). The reason is that these terms would contribute with a factor $(m-1)$ or even $(m-1)(m-2)$. As $q_{i j}^{\text {st(1) }}$ must not depend on $m$, these terms must cancel. On the one hand they do so as we have seen by making use of Wick's theorem. On the other hand, for Gaussian distributed $J_{i j}$ they do not cancel after using Wick's theorem (though the combinations of overlaps are exactly the same). Therefore they already have to cancel in each of the sums $\frac{\lambda^{4}}{24} \sum_{\substack{\alpha, \beta, \gamma, \delta \\ \mu, \nu, \gamma}}^{\mu \nu \eta \rho \rho(0)} q_{\alpha \beta \gamma}^{\mu} \sigma_{\beta}^{\nu} \sigma_{\gamma}^{\eta} \sigma_{\delta}^{\rho}$ and $\frac{\lambda^{4}}{8}\left(\sum_{\mu, \nu}^{\alpha, \beta} q_{\alpha \beta}^{\mu \nu(0)} \sigma_{\alpha}^{\mu} \sigma_{\beta}^{\nu}\right)^{2}$ separately (resp. after taking the Trace over $\sigma_{i}^{s} \sigma_{j}^{t}$ times each single of these sums). We have been able to verify this conjecture, but omit this lengthy calculation.

\section{Replica symmetry}

In the case of replica symmetry we have to calculate equation 4.42 for $i=j$ determining $q_{2}^{(1)}$ and for $i \neq j$ determining $q_{11}^{(1)}$. We find

$$
\begin{aligned}
q_{2}^{(1)} & =\frac{\lambda^{2}}{2}\left(q_{2}^{(1)} q_{4}^{(0)}+(n-1) q_{2}^{(1)} q_{22}^{(0)}+2(n-1) q_{11}^{(1)} q_{31}^{(0)}+(n-1)(n-2) q_{11}^{(1)} q_{211}^{(0)}\right) \\
& -\frac{\lambda^{2}}{2} m n q_{2}^{(0)}\left(q_{2}^{(0)} q_{2}^{(1)}+(n-1) q_{11}^{(0)} q_{11}^{(1)}\right)
\end{aligned}
$$

and

$$
\begin{aligned}
q_{11}^{(1)} & =\frac{\lambda^{2}}{2}\left(2 q_{2}^{(1)} q_{31}^{(0)}+(n-2) q_{2}^{(1)} q_{211}^{(0)}+2 q_{11}^{(1)} q_{22}^{(0)}+4(n-2) q_{11}^{(1)} q_{211}^{(0)}+(n-2)(n-3) q_{11}^{(1)} q_{1111}^{(0)}\right) \\
& -\frac{\lambda^{2}}{2} m n q_{11}^{(0)}\left(q_{2}^{(0)} q_{2}^{(1)}+(n-1) q_{11}^{(0)} q_{11}^{(1)}\right)
\end{aligned}
$$

We insert the zeroth order results and neglect the term proportional to $m n$, as the overlaps must not depend on $n$ (these terms should cancel if we had taken 
terms proportional to $n$ in the first part along). Then, both equations (4.43) and 4.44 simplify to

$$
(2 \lambda-2) q_{11}^{(1)}-(2 \lambda-2) q_{2}^{(1)}=0
$$

We see that $q_{2}^{(1)}=q_{11}^{(1)}$ is the solution. Since there shouldn't be any corrections to the self overlap, $q_{2}^{(1)}$ is zero, and so is $q_{11}^{(1)}$. In the case of Gaussian distributed $J$ in equation (4.7) we find a different result as the terms from equation 4.41) do not cancel by using Wick's theorem but result in a constant $c(\lambda)$ of order 1 in $m$ and $n$.

\section{1-RSB}

The combinatorics are very similar to those in the replica symmetric case, but have to be extended to distinguish overlaps consisting of spins from the same 'replica-block' and from different 'replica-blocks'. Therefore we have to calculate three different overlaps: $q_{2,2}^{(1)}$ for the overlap of the spins $\sigma_{i L}^{s} \sigma_{i L}^{s}$ (the second lower index $L, K=1, \ldots, \frac{n}{r}$ indicates the number of the replica block), $q_{11,2}^{(1)}$ for $\sigma_{i L}^{s} \sigma_{j L}^{s}$ and $q_{11,11}^{(1)}$ for $\sigma_{i L}^{s} \sigma_{j K}^{s}$. This time we find

$$
\begin{aligned}
q_{2,2}^{(1)}= & \frac{\lambda^{2}}{2}\left(q_{2,2}^{(1)} q_{4,4}^{(0)}+q_{2,2}^{(1)}\left((n-r) q_{22,22}^{(0)}+(r-1) q_{22,4}^{(0)}\right)+2(r-1) q_{11,2}^{(1)} q_{31,4}^{(0)}+2(n-r) q_{11,11}^{(1)} q_{31,31}^{(0)}\right. \\
& \quad+q_{11,2}^{(1)}\left((n-r)(r-1) q_{211,22}^{(0)}+(r-1)(r-2) q_{211,4}^{(0)}\right) \\
& \left.+q_{11,11}^{(1)}\left(2(n-r)(r-1) q_{211,31}^{(0)}+(n-r)(n-2 r) q_{211,211}^{(0)}\right)\right) \\
& -\frac{\lambda^{2}}{2} m n q_{2,2}^{(0)}\left(q_{2,2}^{(0)} q_{2,2}^{(1)}+(r-1) q_{11,2}^{(0)} q_{11,2}^{(1)}+(n-r) q_{11,11}^{(0)} q_{11,11}^{(1)}\right)
\end{aligned}
$$

$$
\begin{aligned}
& q_{11,2}^{(1)}=\frac{\lambda^{2}}{2}\left(2 q_{2,2}^{(1)} q_{31,4}^{(0)}+q_{2,2}^{(1)}\left((r-2) q_{211,4}^{(0)}+(n-r) q_{211,22}^{(0)}\right)\right. \\
& +2 q_{11,2}^{(1)} q_{22,4}^{(0)}+4(r-2) q_{11,2}^{(1)} q_{211,4}^{(0)}+q_{11,2}^{(1)}\left((r-2)(r-3) q_{1111,4}^{(0)}+(n-r)(r-1) q_{1111,22}^{(0)}\right) \\
& \left.+4(n-r) q_{11,11}^{(1)} q_{211,31}^{(0)}+q_{11,11}^{(1)}\left(2(r-2)(n-r) q_{1111,31}^{(0)}+(n-r)(n-2 r) q_{1111,211}^{(0)}\right)\right) \\
& -\frac{\lambda^{2}}{2} m n q_{11,2}^{(0)}\left(q_{2,2}^{(0)} q_{2,2}^{(1)}+(r-1) q_{11,2}^{(0)} q_{11,2}^{(1)}+(n-r) q_{11,11}^{(0)} q_{11,11}^{(1)}\right)
\end{aligned}
$$


and

$$
\begin{gathered}
q_{11,11}^{(1)}=\frac{\lambda^{2}}{2}\left(2 q_{2,2}^{(1)} q_{31,31}^{(0)}+q_{2,2}^{(1)}\left(2(r-1) q_{211,31}^{(0)}+(n-2 r) q_{211,211}^{(0)}\right)\right. \\
+4(r-1) q_{11,2}^{(1)} q_{211,31}^{(0)}+q_{11,2}^{(1)}\left(2(r-1)(r-2) q_{1111,31}^{(0)}+(n-2 r)(r-1) q_{1111,211}^{(0)}\right) \\
\quad+2 q_{11,11}^{(1)} q_{22,22}^{(0)}+q_{11,11}^{(1)}\left(4(r-1) q_{211,22}^{(0)}+4(n-2 r) q_{211,211}^{(0)}\right) \\
\left.+q_{11,11}^{(1)}\left(2(r-1)(r-1) q_{1111,22}^{(0)}+4(r-1)(n-2 r) q_{1111,211}^{(0)}+(n-2 r)(n-3 r) q_{1111,1111}^{(0)}\right)\right) \\
-\frac{\lambda^{2}}{2} m n q_{11,11}^{(0)}\left(q_{2,2}^{(0)} q_{2,2}^{(1)}+(r-1) q_{11,2}^{(0)} q_{11,2}^{(1)}+(n-r) q_{11,11}^{(0)} q_{11,11}^{(1)}\right)
\end{gathered}
$$

Since $q_{11,2}^{(0)}=q_{11,11}^{(0)}$ up to order $\frac{1}{m^{2}}$, which implies that there is a single state, resp. replica-block, we can simplify the overlaps $q_{\alpha \beta \gamma \delta, L K M N}$ to $q_{\alpha \beta \gamma \delta}$. With $q_{2,2}^{(1)}=0$, all three equations 4.46)- 4.48) reduce to

$$
(r-1) q_{11,2}^{(1)}=r q_{11,11}^{(1)}
$$

irrespective of the explicit values of $q_{\alpha \beta \gamma \delta}$. The general solution is

$$
\left(\begin{array}{c}
q_{11,2}^{(1)} \\
q_{11,11}^{(1)}
\end{array}\right)=t(r)\left(\begin{array}{c}
1 \\
1-\frac{1}{r}
\end{array}\right)
$$

where $t(r)$ (real number) spans the solution space. To find the value of $r$ and $t$, we have to extremize the free energy $f_{2}$, which we derive by taking the next order in equation (4.12) along, see appendix B.3. This contribution to the free energy can be transformed further by finding all possible combinations in those sums of equation (B.15) which depend linearly on first order overlaps to give $\beta f_{2}^{1-R S B}=\beta f_{2}\left(q^{(0)}\right)+\frac{\lambda^{2}}{4} \sum_{\substack{\alpha, \beta \\ \mu, \nu}}\left(q_{\alpha \beta}^{\mu \nu(1)}\right)^{2}-$ $\frac{\lambda^{4}}{8} \sum_{\substack{\alpha, \beta, \gamma, \delta \\ \mu, \nu, \eta, \rho}}^{\mu \nu(1)} q_{\alpha \beta}^{\eta \rho(1)} q_{\alpha \beta \delta}^{\mu \nu \eta \rho(0)}+\propto\left((r-1) q_{11,2}^{(1)}-r q_{11,11}^{(1)}\right)$ (where $f_{2}\left(q^{(0)}\right)$ denotes the free energy depending only on zeroth order overlaps). The second part is zero according to equation (4.49) (for bimodal $J_{i j}$ ). For Gaussian bonds it is equal to $c(\lambda)$ times the corresponding overlaps in zeroth order. However, in both cases it gives the same contribution as the replica symmetric free energy and we have $\beta f_{2}^{1-R S B}=\beta f_{2}^{R S}+\frac{\lambda^{2}}{4} \sum_{\substack{\alpha, \beta \\ \mu, \nu}}\left(q_{\alpha \beta}^{\mu \nu(1)}\right)^{2}-$ $\frac{\lambda^{4}}{8} \sum_{\substack{\alpha, \beta, \gamma, \delta \\ \mu, \nu, \rho, \rho}}^{\mu \nu(1)} q_{\alpha \beta}^{\eta \rho(1)} q_{\alpha \beta \gamma \delta}^{\mu \nu \eta \rho(0)}$.

Summing over all possible combinations of both remaining sums and inserting the relation $(r-1) q_{11,2}^{(1)}=r q_{11,11}^{(1)}$, we find $\beta f_{2}^{1-R S B}=\beta f_{2}^{R S}$, irrespective of the value of $r$, see appendix B.7.1. We see that the free energy to order $\frac{1}{k^{2}}$ does not change when breaking replica symmetry with the 1-RSB breaking scheme for any block size parameter $r$. 


\section{Gaussian bonds}

Due to different moments of the distribution of the interaction constants $J_{i j}$ of a Gaussian and a $\pm J$-model distribution, in case of choosing a Gaussian distribution, equation (4.9) and the following equation 4.10) change slightly in the prefactor of the four-spin-sum. Therefore using Wick's theorem does not eliminate all terms consisting of four-spin-overlaps as in equation (4.41), but we find

$$
\begin{gathered}
q_{i j}^{s t(1)}=\operatorname{Tr}_{\sigma} \sigma_{i}^{s} \sigma_{j}^{t} \exp \left(\frac{\lambda^{2}}{2} \sum_{\substack{\alpha, \beta \\
\mu, \nu}} q_{\alpha \beta}^{\mu \nu(0)} \sigma_{\alpha}^{\mu} \sigma_{\beta}^{\nu}\right)\left(\frac{\lambda^{2}}{2} \sum_{\substack{\alpha, \beta \\
\mu, \nu}}^{\mu \nu(1)} \sigma_{\alpha \beta}^{\mu} \sigma_{\beta}^{\nu}+\frac{\lambda^{4}}{4}\left(\sum_{\substack{\alpha, \beta \\
\mu, \nu}} q_{\alpha \beta}^{\mu \nu(0)} \sigma_{\alpha}^{\mu} \sigma_{\beta}^{\nu}\right)^{2}\right) \\
\times\left(\operatorname{Tr}_{\sigma} \exp \left(\frac{\lambda^{2}}{2} \sum_{\substack{\alpha, \beta \\
\mu, \nu}} q_{\alpha \beta}^{\mu \nu(0)} \sigma_{\alpha}^{\mu} \sigma_{\beta}^{\nu}\right)\right)^{-1} \\
-q_{i j}^{s t(0)} \operatorname{Tr}_{\sigma} \exp \left(\frac{\lambda^{2}}{2} \sum_{\substack{\alpha, \beta \\
\mu, \nu}} q_{\alpha \beta}^{\mu \nu(0)} \sigma_{\alpha}^{\mu} \sigma_{\beta}^{\nu}\right)\left(\frac{\lambda^{2}}{2} \sum_{\substack{\alpha, \beta \\
\mu, \nu}} q_{\alpha \beta}^{\mu \nu(1)} \sigma_{\alpha}^{\mu} \sigma_{\beta}^{\nu}+\frac{\lambda^{4}}{4}\left(\sum_{\substack{\alpha, \beta \\
\mu, \nu}} q_{\alpha \beta}^{\mu \nu(0)} \sigma_{\alpha}^{\mu} \sigma_{\beta}^{\nu}\right)^{2}\right) \\
\times\left(\operatorname{Tr}_{\sigma} \exp \left(\frac{\lambda^{2}}{2} \sum_{\substack{\alpha, \beta \\
\mu, \nu}} q_{\alpha \beta}^{\mu \nu(0)} \sigma_{\alpha}^{\mu} \sigma_{\beta}^{\nu}\right)\right)^{-1} .
\end{gathered}
$$

Nevertheless, the sums including four-spin overlaps produce only zeroth order contributions in $\frac{1}{k}$, making the homogeneous set of equations 4.42) an inhomogenous one (we again disregard the second part $\sim-q_{i j}^{s t(0)}$ as it is still proportional to $n$.). We omit this paintaking combinatorical exercise and present only the result of it. It is

$$
(2 \lambda-2)\left(\begin{array}{ll}
1 & -1 \\
1 & -1
\end{array}\right)\left(\begin{array}{l}
q_{2}^{(1)} \\
q_{11}^{(1)}
\end{array}\right)=-(2 \lambda-2) c(\lambda)\left(\begin{array}{l}
1 \\
1
\end{array}\right),
$$

with $c(\lambda)=\frac{5}{\lambda}>0$, independent of $r$. We suppose that $q_{2}^{(1)}$ is zero and find $q_{11}^{(1)}=c(\lambda)$, which we can insert into the free energy $\beta f_{2}^{R S \text {,gauss }}$.

In case of 1-RSB the four-spin overlaps in equation (4.51) have the same combinations of zeroth order contributions as for bimodally distributed bonds (which are replica symmetric). Therefore the system of equations (4.46)(4.48) remains unchanged, but there is a common constant at the right hand side (equal to the constant on the right hand side of the replica symmetric calculation, equation 4.52), leading to

$$
(r-1) q_{11,2}^{(1)}=r q_{11,11}^{(1)}+c(\lambda) .
$$


As already pointed out in the computation for bimodally distributed bonds, the free energy is the same as in the replica symmetric case, $\beta f_{2}^{1-R S B \text {,gauss }}=$ $\beta f_{2}^{R S \text {,gauss }}$, irrespective of $r$.

\subsubsection{Discussion}

The purpose of this section was the analytical investigation of the breaking of replica symmetry of the $m$-component spin glass. To this end we have considered two kinds of corrections to the overlaps $q_{\alpha \beta}^{\mu \nu}$ of the fully connected model in the limit $m \rightarrow \infty$, corrections for finite connectivity in $\frac{1}{k}$ and corrections for finite component numbers in $\frac{1}{m}$ and $\frac{1}{m^{2}}$. First of all, we have confirmed the result of Ref. [41] that the fully connected $m$-component spin glass is replica symmetric for $m=\infty$. Unfortunately, we have not found the breaking of replica symmetry for finite component numbers in contrast to this publication. There may be several reasons for this failing. First of all, it might be that we have to go to even higher orders in $\frac{1}{m}$ to find replica symmetry breaking. Another possibility could be that it is necessary to introduce the two-step RSB scheme to improve the results. However, it is striking that it is the expansion of the saddle point equation determining $y$ which forces the parameter $\alpha_{1}(r)$ and $\alpha_{2}(r)$ to be equal to one (as they occur only in the component $L_{x 1}$ of each higher order $x$ (determining $\alpha_{1}, \alpha_{2}$ ), which is calculated from the saddle point equation for $y$ ). It is therefore the starting point of large $m(m \rightarrow \infty$ for which the saddle point equation is derived), which prevents us from observing the breaking of replica symmetry and it is not clear whether it is possible to find replica symmetry breaking by an analytical continuation of the $m \rightarrow \infty$-limit in a power series, as presented in this section. Nevertheless, we are not aware of a method to circumvent starting from this limit. The above suggestions for how to proceed might possibly overcome this problem. It would then be very likely that the corrections in $\frac{1}{k}$ display replica symmetry breaking as well. So far, these corrections are bound to the result in order $\frac{1}{k^{0}}$ of this section, i.e. they do not break replica symmetry. However, they enhance our general knowledge of the free energy of the $m$-component spin glass, depending on the number of components of the spins and on the dimension $d$ of the underlying lattice (which is directly related to the connectivity $k+1$ ), see figure 4.7. We already know that the Ising spin glass $\left(\frac{1}{m}=1\right)$ above six dimensions $\left(\frac{1}{d}=\frac{1}{6}\right)$ is solved by full replica symmetry breaking, see the introduction of this thesis. The same is true for all fully connected $\left(\frac{1}{d}=0\right)$ models with finite component numbers, whereas replica symmetry holds for $m \rightarrow \infty$.

For finite connectivities in this limit we find $f^{R S}=f^{1-R S B}$ for any choice of $r$ (which satistifies $(r-1) q_{11,2}^{(1)}=r q_{11,11}^{(1)}$ ), i.e. the free energy is flat along 


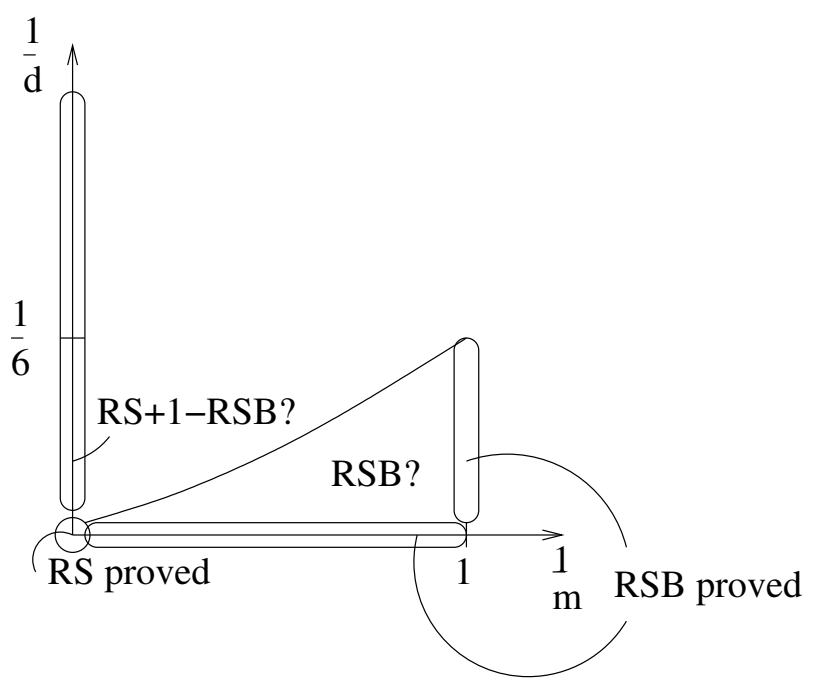

Figure 4.7: Possible "phase diagram" of spin glasses for general $m$-component vector spins concerning the question of stable or broken replica symmetry (depending on the number of components as $\frac{1}{m}$ and the dimension of the underlying lattice as $\frac{1}{d}$ ). The line of separation between RSB and RS is motivated in the text.

the 1-RSB mode. Assuming that this result holds for all orders in $\frac{1}{k}$ and advanced replica symmetry breaking schemes (e.g. 2-RSB), it has interesting consequences. On the one hand it reveals a region of coexistence of replica symmetry and one step broken replica symmetry, which covers at least the $y$-axis in figure 4.7, but possibly extends into the range of finite component numbers to a line of separation between full RSB and the region of coexistence. The argumentation for such a line of separation is motivated by the finding of the upper critical dimension (above which the mean field solution holds) of six for Ising spins and at least eight for the $m$-component spin glass with $m \rightarrow \infty$, see Ref. [44] (but probably higher, see Ref. [50]). In case of Ising spins this means that the mean field solution, which is full broken replica symmetry, might not be the true solution below 6 dimensions. For the large- $m$ limit, the replica symmetric mean field solution could be replaced by the coexistence of replica symmetry and one step broken replica symmetry we have just found. Another consequence of our result is that it forces us to take all saddle points (i.e. all possible solutions for $q_{11,2}^{(1)}$ and $q_{11,11}^{(1)}$ ) along when computing the partition function of the $m$-component spin glass with finite connectivity (which has so far not been achieved). Admitting that this discussion is footed on the assumption that a finite connectivity might stabilize replica symmetry (adapted from the Droplet picture), it offers a new 
perspective to investigate vector spin glasses. 


\section{Chapter 5}

\section{Sample-to-sample Fluctuations of the Free Energy}

As we have seen in the previous chapters, spin glasses of finite size exhibit new phenomena, like a finite number of necessary components to build replica symmetric states in thermal equilibrium. Quite generally, finite size scaling of thermodynamic quantities is worth studying, as real models in experimental samples will always have a finite size. In spin glass physics, the problem of calculating finite size corrections directly can not be solved. This would require to include subdominant orders in the calculation of the partition function, which is impossible, due to the massless modes present in the spin glass phase (see the introduction of this thesis). These modes describe regions of a "flat" mean-field free energy [7], and yield an instability of the mean-field solution at first order in perturbation theory, even in vector spin glasses [42, 44]. Nevertheless, by other analytical and numerical methods there is certain progress of the knowledge of the finite size behaviour of spin glasses. This involves among other things the scaling of the free energy, resp. the ground state energy. In the thermodynamic limit, these energies are self-averaging. This suggests that for instance the free energy of a given disorder configuration and system size $N, F_{J, N}$ scales with the system size as $F_{N}=E_{J} F_{J, N}=N f_{t y p}+f_{1} N^{\Theta_{s}}+\cdots$ (with the shift exponent $\Theta_{s}$ ), and the sample-to-sample-fluctuations scale as $\Delta F_{N}^{2}=E_{J}\left(F_{N}^{2}\right)-\left(E_{J} F_{N}\right)^{2} \sim N^{\mu}$ with the (in general unknown) exponent $\mu$ [58, 59], where $F_{N}=E_{J} F_{J, N}$ is the disorder averaged $\left(E_{J}\right)$ free energy of a spin glass of system size $N, f_{t y p}$ is the typical extensive part of the average free energy and $f_{1}$ is an unspecified constant. These scaling properties describe the leading corrections to the thermodynamic limit for large system sizes $N$. However, corrections of higher order are also present and complicate extracting the scaling exponents from simulations. Recently, the interest in scaling of the free energy fluctuations 
of the SK-Ising spin glass has increased [73, 155, 56, 74, 775, 76, 77, 78, 54]. It is clear that spin glasses do not exhibit Gaussian fluctuations, which would be the case for sums of identically independently distributed random variables, according to the central limit theorem (as the free energies in spin glasses are far from being independent). Within the replica method, the derivation of the free energy fluctuations is in principle possible as can be seen by expanding the logarithm of the $n$-times replicated partition function $\log E_{J}\left(Z^{n}\right)$

$$
\begin{aligned}
\log E_{J}\left(Z^{n}\right) & =\log E_{J}\left(e^{n \log Z}\right)=\log E_{J}\left(1+n \log Z+\frac{n^{2}}{2}(\log Z)^{2}+\cdots\right) \\
= & n E_{J} \log Z+\frac{n^{2}}{2}\left(\left(E_{J} \log Z\right)^{2}-E_{J}(\log Z)^{2}\right)+\cdots \\
& =-n \beta F_{N}+\frac{n^{2}}{2} \beta^{2} \Delta F_{N}^{2}+\cdots
\end{aligned}
$$

It is therefore necessary to extract the second order term in $n$ in the replica formalism, which works successfully in the high temperature phase of the mean-field Ising spin glass [73, 79]. However, in the low temperature phase, the situation is more complex and a direct calculation is not possible.

A different field of active research can be connected to the scaling of finite size corrections in spin glass physics: Extreme value statistics. There, the question of interest is the probability of the occurence of extremal events, such as the maximum (or minimum) value of a set of random numbers, see [80] for an overview. Especially the discovery of the Tracy-Widom distribution of the largest eigenvalue of a Gaussian random matrix [81, where the width of the distribution scales as $N^{1 / 3}$, has intensified investigations. Before, there were three "classical" universality classes of extreme values (the Weibull, Fréchet and Gumbel distribution) and the Tracy-Widom distribution constitutes a new, fourth class. Spin glass physics provide extreme statistics, too. As one is interested in the configuration of spins, which minimizes the ground state energy, the distribution of ground state energies of a certain spin glass model for finite system sizes is a question of extreme value statistics and one may ask whether this distribution falls into any of the known universality classes. For instance, the distribution of ground state energies of the spherical spin glass falls into the Tracy-Widom universality class, as its ground state energy is the smallest eigenvalue of a Gaussian random matrix [38, 59]. The $m$-component spin glass has the same free energy as the spherical model in the thermodynamic limit and it would be interesting to compare their distributions of ground state energies for finite systems. Usually in spin glass physics, the quite formidable task of evaluating the distribution of energies 
is restricted to the analysis of the width of this distribution. As the free energy at $T=0$ corresponds to the ground state energy, we expect the free energies in the spin glass phase to obey the same scaling relation as the ground state energies, although this has never been proved. As the spherical model is replica symmetric, it is interesting to ask whether the free energies of replica symmetric mean-field models have a common universal distribution, as in Ref. [74, and we will be able to deny this question in this chapter by calculating the free energy fluctuations of the $m$-component spin glass in the large- $m$ limit.

A different situation occurs in the case of broken replica symmetry of the Sherrington-Kirkpatrick Ising spin glass, which doesn't fall in any of the four known universality classes. There is growing consensus on the value of $\mu=1 / 6$, following a long debate. On the one hand, this value is supported by replica calculations of the large deviations of the typical free energy [82, 74, 55, 54], which are linked to the small deviations by a matching argument of Ref. [73]. This picture stems from the assumption that the rescaled variable $\left(f-f_{\text {typ }}\right) / \sigma_{N}$ has a limiting distribution in the thermodynamic limit. Large Deviations from the mean free energy are of order 1, i.e. they remain finite in the thermodynamic limit, but have a vanishing weight for large $N$ as the probability of observing them is exponentially small. Small, or typical deviations instead, are observed with a finite probability, and vanish with some subdominant power of $N$. Following the line of argumentation of Ref. [73], it is possible to match both regimes to describe a single probability distribution of the free energy, which gets peaked around the typical value $f_{\text {typ }}$ for large $N$. On the other hand, there are numerical results [60, 58, 54, 55], which are not always clear in their interpretation and do not rule out the value of $1 / 6$. The competing value of $1 / 4$ is supported by heuristic arguments [58], and even more by the numerical results cited above. This states, how difficult it is to find the exact value of scaling exponents by numerics. Furthermore, the upper bound of $\mu \leq \frac{1}{4}$ was analytically derived in Ref. [76, 77]. In contrast to mean-field models (including the $m$-component spin glass, which is the subject of this chapter), the situation is clear for finitely connected spin glasses, which display normal Gaussian fluctuations, as shown in [83]. This homogeneity of a large group of spin glass models was further confirmed by numerics of the diluted Ising spin glass [84] and analytical work [56].

In this chapter, we will adapt the method of [76, 77, 78], developed in the context of Ising spins, to calculate the sample-to-sample-fluctuations of the fully connected $m$-component spin glass in the replica symmetric large- $m$ limit. This method circumvents the direct calculation of the fluctuations via replicas as described above. It is based on the derivation of an exact connec- 
tion between the sample-to-sample-fluctuations and bond chaos. Although there doesn't exist an intuitive explanation for this connection, it is a very useful technique to calculate the free energy fluctuations. Bond chaos [78] means that two replicas with infinitesimally different sets of bonds are totally uncorrelated in the thermodynamic limit, where the link overlap between the two copies measures the degree of correlation. In addition to bond chaos, chaos in temperature has been investigated intensively [85, 86, 87]. Here, we will calculate the finite size corrections to bond chaos, by forcing two replicas to have a certain link overlap different from zero, i.e. to be correlated. We will use the probability distribution of such link overlaps to obtain the free energy fluctuations of the $m$-component spin glass. Fortunately, replica symmetry of the large- $m$ limit simplifies the exact connection in such a way that it is possible to calculate not only an upper bound, as in Ref. [77], but a small interval of possible values of $\mu$.

In the next section, we will show how to derive the exact connection between the fluctuations and bond chaos. Bond chaos will be formulated in terms of the probability distribution of the link overlap $q_{L}$ of two copies of the same spin glass but with possibly different sets of bonds, which is the $\delta$ function $P_{\Delta J}\left(q_{L}\right)=\delta\left(q_{L}\right)$ in the thermodynamic limit (where the two copies with only infinitesimal different sets of bonds $(\Delta J)$ are totally uncorrelated). It is the purpose of this chapter to calculate the finite size scaling of the sample-to-sample fluctuations. Hence, we are interested in the finite size corrections to the formulation of bond chaos in the thermodynamic limit, which is the probability distribution of finding a given link overlap different from zero, and denoted by $P_{\epsilon}\left(q_{L}\right)$, as will become clear below. In order to evaluate the fluctuations, we have to find various moments of the averaged link overlap. Therefore we will calculate the probability distribution $P_{\epsilon}\left(q_{L}\right)$ in the second section by a large deviation approximation of bond chaos and derive these moments from it. In addition to the large deviations results, it will turn out that a part of the sample-to-sample fluctuations is dominated by the small deviations of bond chaos. As we are not able to calculate them directly, we will resort to results from chapter 3, resp. Ref. [48, and use a finite size scaling ansatz for this region in the third section, similar to that in section 4.2. In the last section we will use the results from both regions to calculate the exponent $\mu$ and point out the implications of our result. Although the computation of this chapter involves detailed descriptions of technical steps, the technicalities should not prevent understanding the general steps and the accuracy of the final result. 


\subsection{Exact Connection between the sample- to-sample Fluctuations and Bond Chaos}

We will present two exact connections between the sample-to-sample-fluctuations of the free energy, $\Delta F_{N}$, and bond chaos for the $m$-component spin glass in the large- $m$ limit, the first and second route to chaos, following the derivation of Ref. [77]. We will derive the final result for the second route

$$
\begin{aligned}
\beta^{2} \Delta F_{N}^{2} & =\frac{(N-1)^{2} \beta^{4}}{16} \int_{0}^{\infty} d \epsilon f_{2}(\epsilon)\left(\left[\left(q_{L}^{13}\right)^{2}\right]-\left[q_{L}^{13}\right]^{2}\right)+\frac{(N-1) \beta^{2}}{4} \int_{0}^{\infty} d \epsilon g_{2}(\epsilon)\left[q_{L}^{13}\right] \\
& =: \frac{(N-1)^{2} \beta^{4}}{16} I_{21}+\frac{(N-1) \beta^{2}}{4} I_{22}
\end{aligned}
$$

where $f_{2}(\epsilon)=\frac{2 \epsilon \log \left(1+\epsilon^{2}\right)}{\left(1+\epsilon^{2}\right)^{2}}$ and $g_{2}(\epsilon)=\frac{\epsilon \log \left(1+\epsilon^{2}\right)}{\left(1+\epsilon^{2}\right)^{3 / 2}}$ are nonnegative functions of $\epsilon$ (and $\epsilon$ "measures" the statistical distance between two sets of bonds $\left\{J_{1}\right\}$ and $\left\{J_{3}\right\}$ of two otherwise identical copies 1 and 3 of a spin glass and will be defined below.) The link overlap is $q_{L}^{13}=\frac{1}{N_{B}} \sum_{i<j}\left\langle\vec{s}_{i} \vec{s}_{j}\right\rangle_{1}\left\langle\vec{s}_{i} \vec{s}_{j}\right\rangle_{3}$, with $N_{B}=\frac{N(N-1)}{2}$ in the fully connected model. As the thermal averages $\left\langle\vec{s}_{i} \vec{s}_{j}\right\rangle_{1}$ and $\left\langle\vec{s}_{i} \vec{s}_{j}\right\rangle_{3}$ of both copies may differ, due to the possibly different sets of bonds, the link overlap is a measure of correlation of the two copies 1 and 3 . The notation [...] stands for the average taken with the disorder averaged probability distribution $P_{\epsilon}\left(q_{L}\right)$ of finding a given link overlap $q_{L}$. Next, we will explain in detail where equation 5.2 comes from. To do so, we use the technique of interpolating Hamiltonians [88]. The original Hamiltonian of the SK-model of the $m$-component spin glass is

$$
\mathcal{H}=\sqrt{\frac{1}{N}} \sum_{i<j} J_{i j} \vec{s}_{i} \vec{s}_{j},
$$

where $\vec{s}_{i}$ are $m$-component spins and $J_{i j}$ are independent Gaussian random variables with unit variance. The spins are assumed to be normalized in such a way that ${\overrightarrow{s_{i}}}^{2}=m$. Using two copies of the $m$-component spin glass, each described by an Hamiltonian of equation (5.3) but with independent sets of bonds, the technique of interpolating Hamiltonians allows us to interpolate between the one set of bonds and the other. To derive the second route of chaos ([5.2) ) we have to define two of such interpolating Hamiltonians. In each of them we use a parameter between 0 and 1 , here $t$ and $\tau$, to interpolate between a spin glass with a given set of coupling constants $\left\{J_{i j}\right\}$ for $t=0$ (or $\tau=0$ ) and an identical spin glass with a different, independent set of 
coupling constants $\left\{J_{i j}^{\prime}\right\}$ or $\left\{J_{i j}^{\prime \prime}\right\}$ for $t=1$ (resp. $\left.\tau=1\right)$ :

$$
\begin{aligned}
& \mathcal{H}_{t}=-\sqrt{\frac{1-t}{N}} \sum_{i<j} J_{i j} \vec{s}_{i} \vec{s}_{j}-\sqrt{\frac{t}{N}} \sum_{i<j} J_{i j}^{\prime} \vec{s}_{i} \vec{s}_{j} \text { and } \\
& \mathcal{H}_{\tau}^{\prime}=-\sqrt{\frac{1-\tau}{N}} \sum_{i<j} J_{i j} \vec{s}_{i} \vec{s}_{j}-\sqrt{\frac{\tau}{N}} \sum_{i<j} J_{i j}^{\prime \prime} \vec{s}_{i} \vec{s}_{j} .
\end{aligned}
$$

For all values of $t$ and $\tau$ between 0 and 1, these Hamiltonians describe the same kind of spin glass, but with different bonds $\sqrt{1-t} J_{i j}+\sqrt{t} J_{i j}^{\prime}$ (or $\left.\sqrt{1-\tau} J_{i j}+\sqrt{\tau} J_{i j}^{\prime \prime}\right)$. The bonds are still Gaussian random variables with unit variance and the disorder average taken with respect to all $J$ 's will still be denoted by $E_{J} \cdots$. The difference between $t$ and $\tau$ will be important in the following, as it is a measure of distance between the two sets of bonds of both Hamiltonians. It is this difference which introduces bond chaos.

From each of the interpolating Hamiltonians we can calculate the free energy of the system it describes, as $-\beta F_{t}=\log Z_{t}$ and $-\beta F^{\prime}=\log Z_{\tau}^{\prime}$ with the partition functions $Z_{t}=\operatorname{Tr} \exp \left(-\beta \mathcal{H}_{t}\right)$ and $Z_{\tau}^{\prime}=\operatorname{Tr} \exp \left(-\beta \mathcal{H}_{\tau}^{\prime}\right)$. Based on this, we can express the sample-to-sample-fluctuations of the free energy in terms of the partition functions of the interpolating Hamiltonians, as

$$
\begin{aligned}
& E_{J}\left(\log Z_{1}-\log Z_{0}\right)\left(\log Z_{1}^{\prime}-\log Z_{0}^{\prime}\right)= \\
& E_{J}\left(\log Z_{1} \log Z_{1}^{\prime}-\log Z_{1} \log Z_{0}^{\prime}-\log Z_{0} \log Z_{1}^{\prime}+\log Z_{0} \log Z_{0}^{\prime}\right) \\
&=\beta^{2}\left(\overline{F^{2}}-\bar{F}^{2}\right)=\beta^{2} \Delta F_{N}^{2}
\end{aligned}
$$

where we used the independence of the different sets of bonds to write, for instance, $E_{J} \log Z_{1} \log Z_{0}^{\prime}=E_{J} \beta F_{1} \beta F_{0}^{\prime}=\beta^{2} \bar{F}^{2}$ (the overbar denotes a disorder averaged quantity of the original spin glass of interest, i.e. Eq. (5.3)). On the other hand, the sets at $t=0$ and $\tau=0$ are not independent and yield $E_{J} \log Z_{0} \log Z_{0}^{\prime}=\beta^{2} \overline{F^{2}}$. We further proceed by using the idea of Ref. [88] to represent $\left(\log Z_{1}-\log Z_{0}\right)\left(\log Z_{1}^{\prime}-\log Z_{0}^{\prime}\right)$ by an integral over $t$ and $\tau$

$$
\beta^{2} \Delta F_{N}^{2}=\int_{0}^{1} d t \int_{0}^{1} d \tau E_{J} \frac{\partial}{\partial t} \log Z_{t} \frac{\partial}{\partial \tau} \log Z_{\tau}^{\prime}
$$

and to express the right hand side of equation (5.6) in terms of thermal link averages, like $\left\langle\vec{s}_{i} \vec{s}_{j}\right\rangle$, as in Ref. [77]. We refer the reader to the appendix C 
for a detailed calculation. It results in

$$
\begin{aligned}
& E_{J} \frac{\partial}{\partial t} \log Z_{t} \frac{\partial}{\partial \tau} \log Z_{\tau}^{\prime}= \\
& \frac{\beta^{2}}{16 N^{2}} E_{J} \sum_{i j k l}\left(\left\langle\left(\vec{s}_{i} \vec{s}_{j}\right)\left(\vec{s}_{k} \vec{s}_{l}\right)\right\rangle_{t}-\left\langle\vec{s}_{i} \vec{s}_{j}\right\rangle_{t}\left\langle\vec{s}_{k} \vec{s}_{l}\right\rangle_{t}\right)\left(\left\langle\left(\vec{s}_{i} \vec{s}_{j}\right)\left(\vec{s}_{k} \vec{s}_{l}\right)\right\rangle_{\tau}-\left\langle\vec{s}_{i} \vec{s}_{j}\right\rangle_{\tau}\left\langle\vec{s}_{k} \vec{s}_{l}\right\rangle_{\tau}\right) \\
& \quad+\frac{\beta^{2}}{8 N \sqrt{1-t} \sqrt{1-\tau}}\left(E_{J} \sum_{i j}\left(\left\langle\vec{s}_{i} \vec{s}_{j}\right\rangle_{t}\left\langle\vec{s}_{i} \vec{s}_{j}\right\rangle_{\tau}\right)-m^{2} N\right) .
\end{aligned}
$$

The subscript $t$ or $\tau$ on the thermal averages indicates whether the average is to be taken in a system with Hamiltonian $\mathcal{H}_{t}$ or $\mathcal{H}_{\tau}^{\prime}$. Equation (5.7) involves link overlaps of two replicas, for instance replicas with the label 1 and 3, with possibly different bonds, depending on the explicit values of $t$ and $\tau$, $q_{L}^{13}=\frac{2}{N(N-1)} \sum_{i<j}\left\langle\vec{s}_{i} \vec{s}_{j}\right\rangle_{t}\left\langle\vec{s}_{i} \vec{s}_{j}\right\rangle_{\tau}=\frac{1}{N(N-1)}\left(\sum_{\mu \nu}\left\langle\left(\sum_{i} s_{i \mu}^{(1)} s_{i \nu}^{(3)}\right)^{2}\right\rangle-m^{2} N\right)$ (the latter part is the formulation we will use in the next section). To manipulate equation (5.7) further, we need four different replicas labeled with upper indices (1) to (4) (according to the interpolating parameter, their thermal average is taken with), where replicas 1 and 2 have Hamiltonian $\mathcal{H}_{t}$ and replicas 3 and 4 have Hamiltonian $\mathcal{H}_{\tau}^{\prime}$. With them, the right hand side of equation (5.6) can be expressed in terms of link overlaps of those four replicas

$$
\begin{aligned}
& E_{J} \frac{\partial}{\partial t} \log Z_{t} \frac{\partial}{\partial \tau} \log Z_{\tau}^{\prime}= \\
& \quad \frac{(N-1)^{2} \beta^{4}}{16} E_{J}\left\langle\left(q_{L}^{13}-q_{L}^{23}\right)\left(q_{L}^{13}-q_{L}^{14}\right)\right\rangle+\frac{(N-1) \beta^{2}}{8 \sqrt{1-t} \sqrt{1-\tau}} E_{J}\left\langle q_{L}^{13}\right\rangle
\end{aligned}
$$

So far, the derivation of the second route to chaos does not differ very much from the derivation in Ref. [77] in the context of Ising spins, besides the formulation in terms of the link overlap $q_{L}$. However, in contrast to the Ising spin glass, the $m$-component spin glass in the large- $m$ limit is replica symmetric. If this were not the case, it would be necessary to calculate simultaneous multi-replica overlaps (such as, for instance, the term $E_{J}\left\langle q_{L}^{13} q_{L}^{23}\right\rangle$ ), which was not possible in [77] and restricted that work to calculating only the second part of equation (5.8), resulting in an upper bound for $\mu$ (to be precise: The upper bound was derived using the first route to chaos, but the calculation is similar to here, as will become clear below). The multireplica overlaps must be calculated with the joint probability distribution $P_{\epsilon}^{123}\left(q_{L}^{13}, q_{L}^{23}\right)$ of finding both link overlaps $q_{L}^{13}$ and $q_{L}^{23}$ simultaneously (the parameter $\epsilon$ serves as a "measure" of the statistical distance between the two sets of bonds with interpolation parameter $t$, resp. $\tau$, on which the distribution depends, and will be introduced explicitly in the following). Fortunately, in the case of the $m$-component spin glass this distribution factorizes 
into $P_{\epsilon}^{123}\left(q_{L}^{13}, q_{L}^{23}\right)=P_{\epsilon}\left(q_{L}^{13}\right) P_{\epsilon}\left(q_{L}^{23}\right)$ in the case of replica symmetry, where different replicas are not correlated in any way. This reflects the fact that replicas with the same interpolation parameter will be in the same equilibrium state. A similar statement holds for the four-replica probability distribution $P_{\epsilon}^{1234}\left(q_{L}^{13}, q_{L}^{24}\right)$. Making use of this in equation (5.8), the free energy fluctuations can be written as

$$
\begin{array}{rl}
\beta^{2} \Delta F_{N}^{2}=\frac{(N-1)^{2} \beta^{4}}{16} \int_{0}^{1} d t \int_{0}^{1} & d \tau\left(\left[\left(q_{L}^{13}\right)^{2}\right]-\left[q_{L}^{13}\right]^{2}\right) \\
& +\frac{(N-1) \beta^{2}}{8 \sqrt{1-t} \sqrt{1-\tau}} \int_{0}^{1} d t \int_{0}^{1} d \tau\left[q_{L}^{13}\right] .
\end{array}
$$

The notation $[. .$.$] in the last line stands for the average taken with the bond$ averaged probability distribution $P_{\epsilon}\left(q_{L}\right)$ of finding a given link overlap $q_{L}$. Up to now the overlaps $q_{L}^{13}$ depend on the two parameters $t$ and $\tau$. Since they describe different sets of bonds (the set of bonds for the parameter $t$ in equation (5.4) is $\sqrt{1-t} J_{i j}+\sqrt{t} J_{i j}^{\prime}$, whereas for $\tau$ we have $\sqrt{1-\tau} J_{i j}+\sqrt{\tau} J_{i j}^{\prime \prime}$ ), one may ask if it is possible to express the difference between both sets in a single parameter. As already mentioned, it is possible and the parameter $\epsilon$ measures this distance between both sets of bonds. In Ref. [77] it was shown how to rewrite the configurations of bonds from their original formulation in terms of $t$ and $\tau$ into a formulation solely depending on $\epsilon$ as $\frac{1}{\sqrt{1+\epsilon^{2}}}=$ $\sqrt{1-t} \sqrt{1-\tau}$. In this formulation, the two sets of bonds are identical for $\epsilon=0$ and completely uncorrelated for $\epsilon=\infty$. To derive this simplification, one takes the configuration of bonds from the first of the two interpolating Hamiltonians in equation (5.4) $\mathcal{H}_{t}, K_{i j}^{0}:=\sqrt{1-t} J_{i j}+\sqrt{t} J_{i j}^{\prime}$, to be the reference configuration in the following. The configuration from the second interpolating Hamiltonian, $\mathcal{H}_{\tau}^{\prime}$, is $K_{i j}=\sqrt{1-\tau} J_{i j}+\sqrt{\tau} J_{i j}^{\prime \prime}$. Both $K_{i j}^{0}$ and $K_{i j}$ are Gaussian random variables, since the basic random variables $J_{i j}$, $J_{i j}^{\prime}$ and $J_{i j}^{\prime \prime}$ are Gaussian random variables as well. The correlation of both configurations can be calculated by averaging over the disorder and gives $E_{J} K_{i j}^{0} K_{i j}=\sqrt{1-t} \sqrt{1-\tau}$, due to the independence of $J_{i j}, J_{i j}^{\prime}$ and $J_{i j}^{\prime \prime}$ (such that $E_{J} J_{i j} J_{i j}^{\prime}=0$, for instance). The alternative formulation in terms of $\epsilon$ takes $K_{i j}^{0}$ and an other, independent set $K_{i j}^{\prime}$ as the building blocks of the random variables $K_{i j}(\xi)$ (with an unspecified parameter $\xi$ ). As both $K_{i j}^{0}$ and $K_{i j}^{\prime}$ are Gaussian random variables, $K_{i j}(\xi)$ can be regarded as a possible set of bonds as well

$$
K_{i j}(\xi)=\frac{1}{\sqrt{1+\xi^{2}}} K_{i j}^{0}+\frac{\xi}{\sqrt{1+\xi^{2}}} K_{i j}^{\prime} .
$$

For $\xi=0$, we find $K_{i j}(0)=K_{i j}^{0}$, and for consistency with the first formulation in terms of $t$ and $\tau$, we require $K_{i j}(\xi=\epsilon)$ to be equal to $K_{i j}$ (the set of bonds 
with parameter $\tau$ ), which finally determines $\epsilon$. This is achieved by making both formulations statistically equivalent, which means that the correlation between both configurations of bonds from the first formulation (in terms of $t$ and $\tau$ ) and the second formulation (in terms of $\epsilon$ ) must be equal. As the correlation between $K_{i j}(0)$ and $K_{i j}(\epsilon)$ is $E_{J} K_{i j}(0) K_{i j}(\epsilon)=\frac{1}{\sqrt{1+\epsilon^{2}}}$, we find $\frac{1}{\sqrt{1+\epsilon^{2}}}=\sqrt{1-t} \sqrt{1-\tau}$.

With this relation, we proceed by substituting $\tau$ in favour of $\epsilon$ in equation (5.9). The integrals over $t$ can be solved [77] and the result is equation $(5.2), \beta^{2} \Delta F_{N}^{2}=\frac{(N-1)^{2} \beta^{4}}{16} I_{21}+\frac{(N-1) \beta^{2}}{4} I_{22}$. Finally, the dependence of $q_{L}^{13}$ on $t$ and $\tau$ is replaced by the sole dependence on $\epsilon$. The right hand side of equation (5.2), the second route to chaos, shows the connection to bond chaos, which is represented by $\epsilon$, the measure of distance between two sets of bonds. The following sections answer the purpose of calculating the integrals $I_{21}$ and $I_{22}$. Their indices denote the first and second integral of the second route to chaos.

The derivation of the first route to chaos is similar. Instead of both interpolating Hamiltonians in equation (5.4), we use only the first one, $\mathcal{H}_{t}$. One finds $\Delta F_{N}^{2}=E_{J}\left(\log Z_{1}-\log Z_{0}\right)^{2}($ instead of equation (5.5)). The right hand side is formulated by integral expressions, similar to equation (5.6). It is therefore possible to carry out the same manipulation steps as in the derivation of the second route. The only difference consists in a change of the weight functions $f_{2}(\epsilon)$ and $g_{2}(\epsilon)$ to $f_{1}(\epsilon)=\frac{4 \epsilon^{2}}{\left(1+\epsilon^{2}\right)^{2}} \arcsin \frac{1}{\sqrt{1+\epsilon^{2}}}$ and $g_{1}(\epsilon)=$ $\frac{2}{\left(1+\epsilon^{2}\right)^{3 / 2}} \arcsin \frac{1}{\sqrt{1+\epsilon^{2}}}$, and a minus sign in front of the first integral

$$
\begin{aligned}
\beta^{2} \Delta F_{N}^{2} & =-\frac{(N-1)^{2} \beta^{4}}{16} \int_{0}^{\infty} d \epsilon f_{1}(\epsilon)\left(\left[\left(q_{L}^{13}\right)^{2}\right]-\left[q_{L}^{13}\right]^{2}\right)+\frac{(N-1) \beta^{2}}{4} \int_{0}^{\infty} d \epsilon g_{1}(\epsilon)\left[q_{L}^{13}\right] \\
& =:-\frac{(N-1)^{2} \beta^{4}}{16} I_{11}+\frac{(N-1) \beta^{2}}{4} I_{12} .
\end{aligned}
$$

Again, the functions $f_{1}(\epsilon)$ and $g_{1}(\epsilon)$ are non-negative. Taking the minus sign in front of the first term into account, the leading $N$-dependence of the second term, $\frac{(N-1) \beta^{2}}{4} I_{12}$, is an upper bound for the finite size scaling of the free energy fluctuations (it is possible that the leading orders of both integrals cancel each other). However, as we will not be able to calculate the integrals in detail, we restrict ourselves to calculate the leading orders of the second route to chaos, where both integrals $I_{21}$ and $I_{22}$ exhibit positive contributions. The first step towards this goal is to compute the first and second moment of the probability distribution $P_{\epsilon}\left(q_{L}\right)$ in the next sections. 


\subsection{Calculating $P_{\epsilon}\left(q_{L}\right)$}

We want to calculate the disorder-averaged probability distribution, $P_{\epsilon}\left(q_{L}\right)$, of finding a link overlap $q_{L}$ of a system of two copies of a spin glass, which have possibly different sets of bonds. Again, $\epsilon$ is the measure of that difference. The partition function, $Z_{\epsilon, J}\left(q_{L}\right)$ (defined below), constrains the two copies (or replicas) to have a link overlap $q_{L}$. The system of the two constrained copies has a certain free energy $F_{\epsilon, J}\left(q_{L}\right)$ for a given configuration of bonds, which is calculated as the logarithm of the partition function, as $\beta F_{\epsilon, J}\left(q_{L}\right)=-\log Z_{\epsilon, J}\left(q_{L}\right)$. Therefore, the probability of finding a given link overlap in such a system is proportional to the Boltzmannweight $\exp \left(-\beta F_{\epsilon, J}\left(q_{L}\right)\right)$. Each of the two copies is described by the same Hamiltonian but with different sets of bonds $K_{i j}(0)$ and $K_{i j}(\epsilon)$ from equation (5.10). Constraining two replicas to have a certain overlap was first suggested in Ref. [89] and forces the replicas to differ from their thermal state in the unconstrained case in the thermodynamic limit (which, in contrast, demands $\left.q_{L}=0\right)$. The constraint is implemented using the $\delta$-function $\delta\left(q_{L}-\frac{1}{N(N-1)} \sum_{\mu \nu}\left(\sum_{i} s_{i \mu}^{(10)} s_{i \nu}^{(3 \epsilon)}\right)^{2}-\frac{m^{2}}{N-1}\right)$, where the upper indices denote the replica number in its first entry and the value of $\epsilon$ in its second entry. The partition function $Z_{\epsilon, J}\left(q_{L}\right)$ of the system of two copies of the same spin glass then is

$$
\begin{array}{r}
Z_{\epsilon, J}\left(q_{L}\right)=\operatorname{Tr}_{\vec{s}}\left(\delta\left(q_{L}-\frac{1}{N(N-1)} \sum_{\mu \nu}\left(\sum_{i} s_{i \mu}^{(10)} s_{i \nu}^{(3 \epsilon)}\right)^{2}-\frac{m^{2}}{N-1}\right)\right) \\
\quad \times \exp \left(\frac{\beta}{\sqrt{N}} \sum_{i<j} K_{i j}(0){\overrightarrow{s_{i}}}^{(10)}{\overrightarrow{s_{j}}}^{(10)}+\frac{\beta}{\sqrt{N}} \sum_{i<j} K_{i j}(\epsilon){\overrightarrow{s_{i}}}^{(3 \epsilon)}{\overrightarrow{s_{j}}}^{(3 \epsilon)}\right) .
\end{array}
$$

The non-disorder averaged probability distribution $P_{\epsilon, J}\left(q_{L}\right)$ follows to be

$$
P_{\epsilon, J}\left(q_{L}\right)=\frac{\exp \left(-\beta F_{\epsilon, J}\left(q_{L}\right)\right)}{\int_{0}^{\infty} d q_{L}^{\prime} \exp \left(-\beta F_{\epsilon, J}\left(q_{L}^{\prime}\right)\right)} .
$$

If we were able to calculate the free energy $F_{\epsilon, J}\left(q_{L}\right)$ and average equation (5.13) over the disorder, this would give the desired probability distribution $P_{\epsilon}\left(q_{L}\right)$. Unfortunately, calculating the partition function $Z_{\epsilon, J}\left(q_{L}\right)$ is too difficult in general, as already pointed out. Therefore, we restrict the computation to the feasible case of calculating the extensive part of the average free energy, $f_{\epsilon}\left(q_{L}\right)$, using replica methods. This free energy is the first part in the separation $F_{\epsilon, J}\left(q_{L}\right)=N m f_{\epsilon}\left(q_{L}\right)+\Delta F_{\epsilon}\left(q_{L}\right)+\Delta F_{\epsilon, J}\left(q_{L}\right)$ of the free energy $F_{\epsilon, J}\left(q_{L}\right)$ of a system with a given configuration of disorder. The second part, $\Delta F_{\epsilon}\left(q_{L}\right)$, denotes the finite size corrections to the disorder 
averaged free energy $E_{J} F_{\epsilon, J}\left(q_{L}\right)$. They scale with some subdominant power of the system size $N$, as $N^{\Theta_{s}}$, where the shift exponent $\Theta_{s}$ is smaller than unity. The last part $\Delta F_{\epsilon, J}\left(q_{L}\right)$ comes from the sample-to-sample deviations of the free energy about its disorder average $F_{\epsilon}\left(q_{L}\right)=E_{J} F_{\epsilon, J}\left(q_{L}\right)$, implying $E_{J} \Delta F_{\epsilon, J}\left(q_{L}\right)=0$. Approximating the free energy of a given configuration of disorder by the extensive part of the free energy is the large deviations formulation, i.e. those deviations that remain finite for large $N$ (and therefore have an exponentially vanishing probability for $N \rightarrow \infty$ ). In other words, the link overlap $q_{L}$ is strictly zero in the thermodynamic limit, but for finite $N$ we force $q_{L}$ to differ from zero. The probability of observing such link overlaps can only be approximated with the extensive part of the free energy, which describes the large deviations of $q_{L}=0$. The probability distribution of finding a given link overlap within this approximation is

$$
P_{\epsilon}^{0}\left(q_{L}\right)=\frac{\exp \left(-\beta N m f_{\epsilon}\left(q_{L}\right)\right)}{\int_{0}^{\infty} d q_{L}^{\prime} \exp \left(-\beta N m f_{\epsilon}\left(q_{L}^{\prime}\right)\right)}
$$

Mean, $\left[q_{L}\right]_{0}$, and variance, $\left[q_{L}^{2}\right]_{0}-\left[q_{L}\right]_{0}^{2}$, of this distribution have the index 0 , and replace $\left[q_{L}\right]$ and $\left[q_{L}^{2}\right]-\left[q_{L}\right]^{2}$ in the exact connection between the free energy fluctuations and bond chaos in the equations (5.2) and (5.11) in those regions where the large deviations approximation is valid (which we will find out later).

Since the $m$-component spin glass is replica symmetric in the large- $m$ limit, each replicated copy of it with the same set of bonds will be in the same equilibirum state. Hence, the probability distribution for $\epsilon=0$, i.e. for identical sets of bonds $K_{i j}(\epsilon)$, consists of a single $\delta$-peak at the EdwardsAnderson-value of $q_{L}$ in the thermodynamic limit. As we will see, the large deviations calculation does not display this peak. This implies that it is caused by the finite size corrections $\Delta F_{\epsilon}\left(q_{L}\right)$, the small deviations. As the $\delta$ peak (or the finite size behaviour of it) is the dominant part of the distribution for small $\epsilon$ (in fact, for $\epsilon=0$ it is the only one), but the large deviations describe the region of larger $\epsilon$, we expect a crossover value from one part to the other. Finding this value involves knowing the finite size scaling of the small deviations of the free energy. Fortunately, we have investigated this problem at $T=0$ in an earlier work in Ref. [48], which is described in chapter 3. We will implement the behaviour for small $\epsilon$ with a scaling ansatz, which follows from the dominance of the small deviations in that region, after the calculation of the large deviations approximation in the next section. 


\subsubsection{Replica calculation}

The replica trick allows for the calculation of the disorder averaged free energy in the thermodynamic limit [4] by taking the logarithm of the disorder average of the partition function of $n$ uncoupled replicas of a given problem. The linear term in $n$ gives the free energy, see equation (5.1). In our case this means $N m f_{\epsilon}\left(q_{L}\right)=\lim _{n \rightarrow 0} \frac{\log E_{J}\left(Z_{\epsilon, J}\left(q_{L}\right)\right)^{n}}{n}$ with the partition function $Z_{\epsilon, J}\left(q_{L}\right)$ from equation (5.12). The calculation of the disorder averaged, $n$-times replicated partition function of a single $m$-component spin glass has been achieved in [44, and we adapt this computation for our purposes, presenting only the most necessary parts of it. We refer the reader to a detailed calculation of the partition function in appendix $\mathrm{D}$. The first step is to average $\left(Z_{\epsilon, J}\left(q_{L}\right)\right)^{n}=\prod_{\alpha=1}^{n} Z_{\epsilon, J, \alpha}\left(q_{L}\right)$ (where $\alpha$ is the replica index of the spins) over the disorder $E_{J}$, which takes the independent, basic sets of disorder $K_{i j}^{0}$ and $K_{i j}^{\prime}$ from equation (5.10) to be Gaussian with unit variance. Then, we use an integral representation of the $\delta$-functions, $\prod_{\alpha} \delta\left(q_{L}-\frac{1}{N(N-1)} \sum_{\mu \nu}\left(\sum_{i} s_{i \alpha \mu}^{(10)} s_{i \alpha \nu}^{(2 \epsilon)}\right)^{2}-\frac{m^{2}}{N-1}\right)$, with parameters $z_{\alpha}$ (for each replica $\alpha=1, \ldots, n)$, followed by a Hubbard-Stratonovich transformation of the spin variables, which introduces the fields $Q_{\alpha \beta}^{\mu \nu(10)}, Q_{\alpha \beta}^{\mu \nu(3 \epsilon)}$ and $R_{\alpha \beta}^{\mu \nu}$ $(\alpha, \beta=1, \ldots, n$ and $\mu, \nu=1, \ldots, m)$. They can be interpreted as an expectation value of two components $\mu$ and $\nu$ of a spin from possibly different replicas $\alpha$ and $\beta$ (of the same or different copies 1, resp. 3) $Q_{\alpha \beta}^{\mu \nu(10)}=\left\langle s_{\alpha \mu}^{(10)} s_{\beta \nu}^{(10)}\right\rangle$ (and analoguoulsy for $(10) \rightarrow(3 \epsilon)$ ), resp. $R_{\alpha \beta}^{\mu \nu}=\left\langle s_{\alpha \mu}^{(10)} s_{\beta \nu}^{(3 \epsilon)}\right\rangle$. We see, that $Q_{\alpha \beta}^{\mu \nu}$ corresponds to the overlap $q_{\alpha \beta}^{\mu \nu(0)}$ from section 4.3 .

Proceeding further, we separate both tensors $Q_{\alpha \beta}^{\mu \nu}$ (for both copies (10), $(2 \epsilon)$ ) into its diagonal and off-diagonal part by introducing the traceless tensor $T_{\alpha}^{\mu \nu}$ (with $\sum_{\substack{\alpha, \beta \\ \mu, \nu}} Q_{\alpha \beta}^{\mu \nu}=2 \sum_{\substack{\alpha<\beta \\ \mu, \nu}} Q_{\alpha \beta}^{\mu \nu}+2 \sum_{\alpha, \mu<\nu} T_{\alpha}^{\mu \nu}+\sum_{\alpha, \mu}\left(Q_{\alpha \alpha}+T_{\alpha}^{\mu \mu}\right)[43$, 44]). The resulting Gaussian integrals for both $Q_{\alpha \alpha}$ can be solved. Expanding the remaining exponential in a power series up to third order in the tensors $Q, T$ and $R$, we can take the Trace over the spins using spherical coordinates. The expansion in terms of small $Q, T$ and $R$ is valid close to the critical 
temperature. Re-exponentiating the power series yields

$$
\begin{gathered}
E_{J} Z_{\epsilon, J}^{n}\left(q_{L}\right) \propto \int\left(\prod_{\alpha} d z_{\alpha}\right) e^{-N \sum_{\alpha} q_{L} z_{\alpha}-m^{2} \sum_{\alpha} z_{\alpha}} \int d \Lambda_{\alpha \beta}^{\mu \nu} \exp \left(N \left[\tau \sum_{\substack{\alpha<\beta \\
\mu, \nu}}\left(Q_{\alpha \beta}^{\mu \nu}\right)^{2}\right.\right. \\
+\left(\tau+\frac{r}{m+2}\right) \sum_{\alpha, \mu<\nu}\left(T_{\alpha}^{\mu \nu}\right)^{2}+\left(\tau+\frac{r^{\prime}}{m+2}\right) \sum_{\alpha, \mu}\left(T_{\alpha}^{\mu \mu}\right)^{2}+\tau^{\prime} \sum_{\substack{\alpha \neq \beta \\
\mu, \nu}}\left(R_{\alpha \beta}^{\mu \nu}\right)^{2} \\
+\sum_{\alpha, \mu, \nu}\left(\frac{1}{2}-\frac{1}{2 \beta^{2}}\left(\frac{2 z_{\alpha}}{\beta^{2}}+\frac{1}{\sqrt{1+\epsilon^{2}}}\right)^{(-1)}\right)\left(R_{\alpha \alpha}^{\mu \nu}\right)^{2}+\frac{\omega}{3 !}\left(\sum_{\substack{\alpha<\beta<\gamma \\
\mu, \nu, \rho}} Q_{\alpha \beta}^{\mu \nu} Q_{\beta \gamma}^{\nu \rho} Q_{\gamma \alpha}^{\rho \mu}\right. \\
+\frac{m^{2}}{(m+2)(m+4)} \sum_{\substack{\alpha \\
\mu<\nu<\rho}} T_{\alpha}^{\mu \nu} T_{\alpha}^{\nu \rho} T_{\alpha}^{\rho \mu}+\frac{15 m^{2}}{(m+2)(m+4)} \sum_{\alpha, \mu}\left(T_{\alpha}^{\mu \mu}\right)^{3} \\
+\frac{3 m}{m+2} \sum_{\substack{\alpha<\beta \\
\nu, \mu<\rho}} Q_{\alpha \beta}^{\mu \nu} Q_{\beta \alpha}^{\nu \rho} T_{\alpha}^{\rho \mu}+3 \sum_{\substack{(\alpha<\beta) \neq \gamma \\
\mu, \nu, \rho}} Q_{\alpha \beta}^{\mu \nu} R_{\beta \gamma}^{\nu \rho} R_{\gamma \alpha}^{\rho \mu}+3 \sum_{\substack{\alpha<\beta \\
\mu, \nu, \eta}} Q_{\alpha \beta}^{\mu \nu} R_{\beta \alpha}^{\nu \eta} R_{\alpha \alpha}^{\eta \mu} \\
\left.\left.\left.+\frac{3 m}{m+2} \sum_{\substack{\alpha \neq \beta \\
(\mu<\nu), \rho}} T_{\alpha}^{\mu \nu} R_{\alpha \beta}^{\nu \rho} R_{\beta \alpha}^{\rho \mu}+3 \sum_{\substack{\alpha \\
\mu<\nu, \eta}} T_{\alpha}^{\mu \nu} R_{\alpha \alpha}^{\nu \eta} R_{\alpha \alpha}^{\eta \mu}\right)\right]\right), \quad(5.15)
\end{gathered}
$$

with $\tau=\frac{1}{2}\left(1-\frac{1}{\beta^{2}}\right), \tau^{\prime}=\frac{1}{2}\left(1-\frac{\sqrt{1+\epsilon^{2}}}{\beta^{2}}\right), r=-1, r^{\prime}=2 m-1, \omega=1$ and the matrix $\Lambda_{\alpha \beta}^{\mu \nu}$ is given by

$$
\Lambda=\left(\begin{array}{cc}
Q^{(10)} & R \\
R & Q^{(2 \epsilon)}
\end{array}\right)
$$

with the above separation for both $Q_{\alpha \beta}^{\mu \nu}$ and only $T_{\alpha}^{\mu \mu}$ on the diagonal of the matrices $Q$. We further separate the diagonal part $p_{d}^{\mu \nu}=R_{\alpha \alpha}^{\mu \nu}$ (i.e. where the replica indices are identical) of the tensor $R_{\alpha \beta}^{\mu \nu}$ from the rest of it with $\alpha \neq \beta$. The simplification resulting from it will become apparent in the next section. We solve this equation by calculating the saddle points of the exponential with respect to the tensors $Q_{\alpha \beta}^{\mu \nu}, T_{\alpha}^{\mu \nu}$ and $R_{\alpha \beta}^{\mu \nu}$ and the parameters $z_{\alpha}$. This task will be simplified by the symmetry of replicas of the $m$-component spin glass in the next section.

\subsection{Solving the Saddle Point Equations}

The saddle points of equation (5.15) determine the free energy $f_{\epsilon}\left(q_{L}\right)$ and the probability distribution $P_{\epsilon}^{0}\left(q_{L}\right)$. With the probability distribution, we will be able to calculate the mean, $\left[q_{L}\right]_{0}$, and the variance, $\left[q_{L}^{2}\right]_{0}-\left[q_{L}\right]_{0}^{2}$, of 
$q_{L}$. This quantities will finally reveal the finite size scaling of the free energy fluctuations via equation (5.2) and (5.11). As replica symmetry holds in the large- $m$ limit, we can make the ansatz

$$
\begin{array}{ll}
Q_{\alpha \beta}^{\mu \nu}=Q \delta_{\mu \nu}, & T_{\alpha}^{\mu \nu}=0 \\
R_{\alpha \beta}^{\mu \nu}=P \delta_{\mu \nu}, & p_{d}^{\mu \nu}=p_{d} \delta_{\mu \nu} \quad \text { and } \quad z_{\alpha}=z .
\end{array}
$$

Since we have the relation $Q_{\alpha \beta}^{\mu \nu}=\left\langle s_{\alpha \mu}^{(10)} s_{\beta \nu}^{(10)}\right\rangle$ (and similar relations for $T$ and $R$ ) from above and assume isotropy of the lattice, we can neglect correlations between different components $\mu, \nu$ [41, 44]. Furthermore, the tensor $T_{\alpha}^{\mu \nu}$ was chosen to be traceless, which implies $T_{\alpha}^{\mu \mu}=0$, due to the assumption of isotropy.

The partition function simplifies to

$$
\begin{gathered}
E_{J} Z_{\epsilon, J}^{n}\left(q_{L}\right) \propto \int\left(\frac{N-1}{2 \pi}\right)^{n} d z e^{-2 N n q_{L} z-m^{2} n z} \int d \Lambda_{\alpha \beta}^{\mu \nu} \exp \left(N \left[\tau m n(n-1) Q^{2}\right.\right. \\
+\tau^{\prime} m n(n-1) P^{2}+\frac{m n}{2} p_{d}^{2}-m n \frac{1}{2 \beta^{2}}\left(\frac{2 z}{\beta^{2}}+\frac{1}{\sqrt{1+\epsilon^{2}}}\right)^{(-1)} p_{d}^{2}+2 \frac{\omega m n(n-1)(n-2)}{3 !} Q^{3} \\
\left.\left.+\omega m n(n-1)(n-2) Q P^{2}+2 \omega m n(n-1) Q P p_{d}\right]\right) .
\end{gathered}
$$

We see that the exponent is proportional to $N m$, which serves as the large parameter in the following saddle point calculation. Differentiating the exponent with respect to each of the various integration variables yields the saddle point equations

$$
\begin{aligned}
0 & =\tau Q-Q^{2}-P^{2}+P p_{d} \\
0 & =\tau^{\prime} P-2 Q P+Q p_{d} \\
0 & =p_{d}-\frac{1}{\beta^{2}} p_{d}\left(\frac{2 z}{\beta^{2}}+\frac{1}{\sqrt{1+\epsilon^{2}}}\right)^{(-1)}-2 Q P \\
\frac{q_{L}}{m} & =\left(\frac{p_{d}}{\beta^{2}\left(\frac{2 z}{\beta^{2}}+\frac{1}{\sqrt{1+\epsilon^{2}}}\right)}\right)^{2},
\end{aligned}
$$

where line 3 and line 4 can be combined to

$$
p_{d}-2 Q P=\sqrt{\frac{q_{L}}{m}} .
$$

For convenience, we substitute $\sqrt{\frac{q_{L}}{m}}$ by $q$. In terms of $q$, the free energy $f_{\epsilon}(q)$ (which is the replica limit $n \rightarrow 0$ of $\frac{\log E_{J}\left(Z_{\epsilon, J}(q)\right)^{n}}{n}$, the logarithm of equation (5.18), is

$\beta f_{\epsilon}(q)=$ const. $+p_{d} q-\frac{p_{d}^{2}}{2}-\frac{q^{2}}{2\left(1-2 \tau^{\prime}\right)}+\tau Q^{2}+\tau^{\prime} P^{2}-\frac{2}{3} Q^{3}-2 Q P^{2}+2 Q P p_{d}$. 
We proceed by solving the saddle point equations (5.19), (5.20) and (5.23) above, at and below the critical temperature. These solutions will be used in the last section of this chapter to calculate the scaling behaviour of the free energy fluctuations via the exact connection to bond chaos.

\subsubsection{Above and at the critical temperature}

Above $(\tau<0)$ and at the critical temperature $(\tau=0)$, the Ising spin glass is replica symmetric, just as the $m$-component spin glass. Therefore the solutions of the saddle point equations in Ref. [78] apply in our case, too. Above $T_{c}$ and for $q=0$, they are $Q=0, P=0$ and $p_{d}=0$. For $q \neq 0$, we find the solution for small $q$ by noticing that it is not possible to fulfill the saddle point equations with the ansatz $Q=\mathcal{O}(q)$ and $P=\mathcal{O}(q)$. Hence, in first order in $q$, the solution is $p_{d}=q+O\left(q^{4}\right), Q=O\left(q^{2}\right)$ and $P=O\left(q^{2}\right)$. Even more, for $Q$ and $P$ of order $q^{2}$, it is neither possible to fulfill the saddle point equations. Therefore, the final solution is $p_{d}=q+o\left(q^{4}\right), Q=o\left(q^{2}\right)$ and $P=o\left(q^{2}\right)$ (and that is all we need to know, as the free energy in that case is dominated by the terms coming from $p_{d}$ ). Inserting this solution into the free energy (equation (5.24) ) gives

$$
\beta f_{\epsilon}(q)=\frac{q^{2}}{2}\left(1-\frac{\beta^{2}}{\sqrt{1+\epsilon^{2}}}\right)+\mathcal{O}\left(q^{4}\right)
$$

At $T_{c}$, which implies $\tau=0$, the probability distribution $P_{\epsilon}(q) \propto e^{-N m \beta f_{\epsilon}(q)}$ consists of two parts with different dominating exponents depending on $\epsilon$ being smaller or larger than $N^{-1 / 6}$

$$
P_{\epsilon}(q) \propto\left\{\begin{array}{ll}
e^{-N m \frac{q^{3}}{6}} & \epsilon \ll N^{-1 / 6} \\
e^{-N m \frac{q^{2} \epsilon^{2}}{4}} & N^{-1 / 6} \ll \epsilon
\end{array} .\right.
$$

We understand this by noticing that for $q \ll \tau^{\prime}$, i.e. $q \ll \epsilon^{2} \ll 1$, we find the solution from above the critical temperature, $\beta f_{\epsilon}(q)=\frac{q^{2}}{2}\left(1-\frac{\beta^{2}}{\sqrt{1+\epsilon^{2}}}\right)+\mathcal{O}\left(q^{4}\right)$. The solution in the different limit $\epsilon^{2} \ll q \ll 1$ is $\beta f_{\epsilon}(q)=\frac{1}{6} q^{3}+\frac{3 \epsilon^{2}}{16} q^{2}+$ $\mathcal{O}\left(\epsilon^{4}, q^{4}\right)$, see Ref. [78]. The crossover value of $N^{-1 / 6}$ in the separation of the free energy stems from the fact that in both cases, the dominating part of $P_{\epsilon}^{0}(q) \sim e^{-N m f_{\epsilon}(q)}$ has an exponent of order 1 . In the crossover region, $\epsilon^{2} \approx q$, we therefore find $\epsilon=\mathcal{O}\left(N^{-1 / 6}\right)$. Below $N^{-1 / 6}$, the solution for $\epsilon^{2} \ll q \ll 1$ is dominating, while above $N^{-1 / 6}$, the other limit, $q \ll \epsilon^{2} \ll 1$, applies.

From these results, the same free energy fluctuations follow as in the Ising 
spin glass, see Ref. [54]. They are

$$
\begin{array}{ll}
\Delta F_{N} & =-\frac{1}{2} \log \left(1-\beta^{2}\right)-\frac{\beta^{2}}{2}+\mathcal{O}(1 / N) \quad \text { above } T_{c} \\
\Delta F_{N} & =\frac{1}{6} \log N+\mathcal{O}(1) \quad \text { at } T_{c} .
\end{array}
$$

\subsubsection{Below the critical temperature}

We can not solve the saddle point equations for general $q$ and $\epsilon$ (where $\epsilon$ is solely contained in $\tau^{\prime}$ ). Due to this, we derive the solutions for $q=0$ and $\epsilon=0$ and calculate corrections to them perturbatively. The exact solution for $q=0$ is

$$
\begin{aligned}
P & =0 \\
Q & =\tau \\
p_{d} & =0 .
\end{aligned}
$$

By the way, the solution linear in $T$ for $Q$ is $1-T$, which is consistent with our result for $q_{11}^{(0)}\left\langle s_{\alpha}^{\mu} s_{\beta}^{\mu}\right\rangle$ from section 4.3 .

To find the solution for $\epsilon=0$, we rewrite the saddle point equations (5.19) and (5.20) in terms of the new variables $a=Q+P$ and $b=Q-P$

$$
\begin{aligned}
& \left(\tau+p_{d}\right) a-\frac{\tau-\tau^{\prime}}{2}(a-b)-a^{2}=0 \\
& \left(\tau-p_{d}\right) b+\frac{\tau-\tau^{\prime}}{2}(a-b)-b^{2}=0
\end{aligned}
$$

For $\epsilon=0$, implying $\tau=\tau^{\prime}$, they have the solution $a_{0}=\tau+p_{d}$ and $b_{0}=\tau-p_{d}$ (for $a, b \neq 0$ ) and therefore

$$
\begin{aligned}
& Q=\tau \\
& P=p_{d}=\frac{q}{1-2 \tau} .
\end{aligned}
$$

With equation 5.24 we get $\beta f_{0}(q)=\beta f_{0}=\frac{1}{3} \tau^{3}$. We will use this free energy as the reference free energy, as $\epsilon=0$ implies that both copies have identical bonds, and it is the free energy of two unconstrained systems. Taking the possibility of $b=0$ into account (the solution with $a=0$ is an unphysical one), this solution (with $a=\tau+p_{d}$ ) maximizes the free energy for $q>\tau-$ $2 \tau^{2}:=q_{E A}$, instead of the solution with $b=\tau-p_{d}$. In terms of $\Delta q=q-q_{E A}$ 
it is (to lowest order in $\Delta q$ )

$$
\begin{gathered}
Q=P=\frac{\tau+p_{d}}{2} \\
p_{d}=\tau+\frac{\Delta q}{1-2 \tau},
\end{gathered}
$$

and the difference to $\beta f_{0}$ is

$$
\Delta f_{0}(q)=c_{0} \Delta q^{3}
$$

with $c_{0}=\frac{1}{6(1-2 \tau)^{3}}$. The probability distribution of the large deviations approximation of finding a given link overlap $q_{L}$ for $\epsilon=0$ then is

$$
P_{0}^{0}(q)=\left\{\begin{array}{l}
\text { const. } q \in\left[0, q_{E A}\right] \\
e^{-N m c_{0} \Delta q^{3}} \text { otherwise. }
\end{array}\right.
$$

However, in the thermodynamic limit the true probability distribution $P_{0}(q)$ for $\epsilon=0$ consists of a $\delta$-peak at the replica symmetric link overlap $q_{E A}$. The finite size corrections to this peak should be the dominating part of $P_{0}(q)$. As the large deviations approximation does not display this behaviour, see equation (5.36), we conclude that the $\delta$-peak at $q_{E A}$ is generated by the finite size corrections to this approximation, the small deviations. Unfortunately, we are not able to calculate them. Therefore we have to resort to other methods to estimate them and find the value of $\epsilon$ up to which the small deviations are the dominating part of the probability distribution $P_{\epsilon}(q)$ (as we expect the dominance of small deviations to carry over for small non-zero $\epsilon)$. This will be done later. First we will calculate corrections to the first solution of the large deviations approximation, $f_{0}$, in various different limits of finite $\epsilon$ and $q$.

\section{Solution for $\epsilon \rightarrow \infty$}

This limit, or equivalently $\tau^{\prime} \rightarrow-\infty$, corresponds to uncorrelated bonds between copy 1 and 3 . To find the free energy in this case we take the leading order (in $\tau^{\prime}$ ) of equation (5.20)

$$
P=-\frac{Q p_{d}}{\tau^{\prime}}+\mathcal{O}\left(\frac{1}{\left(\tau^{\prime}\right)^{2}}\right),
$$

insert it into equation 5.19 and find (again in leading order)

$$
0=\left(\tau+\frac{p_{d}^{2}}{\tau^{\prime}}\right) Q-Q^{2}
$$


For $\tau^{\prime}=-\infty, Q=\tau, P$ is zero and $p_{d}$ is equal to $q$. The free energy difference to $f_{0}$ (the unconstrained case) is

$$
\beta f_{\infty}(q)-\beta f_{0}=q p_{d}-\frac{p_{d}^{2}}{2}+\mathcal{O}\left(p_{d}^{4}\right)=\frac{q^{2}}{2}+\mathcal{O}\left(q^{4}\right)
$$

Perturbative solution for $\epsilon^{2} \ll q \ll 1$

In this limit, it is useful to apply the saddle point equations in terms of $a$ and $b$, equation (5.30) and (5.31). We take the solution found for $\epsilon=0$ as the undisturbed solution $a_{0}$ and $b_{0}$ (resp. $Q_{0}, P_{0}$ and $p_{d 0}$ from equations (5.32) and (5.33) $)$ and insert $a=a_{0}+a_{1}$ and $b=b_{0}+b_{1}$, where the index 1 denotes the first order corrections for small $\epsilon$. We get (with $\Delta \tau=\tau-\tau^{\prime}>0$ )

$$
\begin{gathered}
a_{1}=-\frac{\Delta \tau p_{d 0}-p_{d 1} a_{0}}{\tau+p_{d 0}} \\
b_{1}=\frac{\Delta \tau p_{d 0}-p_{d 1} b_{0}}{\tau-p_{d 0}}
\end{gathered} \Rightarrow \begin{gathered}
Q_{1}=\frac{\epsilon^{2} q^{2}}{4 \tau^{2}(1-2 \tau)} \\
P_{1}=-\frac{\epsilon^{2} q}{4 \tau}
\end{gathered}
$$

and the correction to $p_{d}$ is $p_{d 1}=-\frac{\epsilon^{2} q}{2}$. Plugging these results into equation (5.24) for the free energy, the difference to the unconstrained solution, $f_{0}$, is

$$
\beta f_{\epsilon}(q)-\beta f_{0}=\frac{2 \tau+1}{16 \tau} \epsilon^{4} q^{2}+\mathcal{O}\left(\epsilon^{4} q^{4}\right)
$$

Perturbative solution for $q \ll \epsilon^{2} \ll 1$

The suitable reference solution in this limit is the one with $q=0$, equations (5.29). We introduce corrections for small $q$ to this solution as $Q \rightarrow \tau+\Delta Q$, $P \rightarrow 0+\Delta P$ and $p_{d} \rightarrow 0+\Delta p_{d}$. Equation (5.20) then follows to be (in lowest order) $\tau^{\prime} \Delta P-2 Q \Delta P+Q \Delta p_{d}=0$. Combined with equation (5.23) we find

$$
\tau^{\prime} \Delta P-2 Q \Delta P+Q(q+2 Q \Delta P)=0
$$

where $q$ is treated as a first order perturbation as well. The solution for $\Delta P$ is

$$
\Delta P=-\frac{\tau q}{\tau^{\prime}-2 \tau+2 \tau^{2}} \stackrel{\epsilon^{2} \ll 1}{\rightarrow} \frac{q}{1-2 \tau}-\frac{\epsilon^{2} q}{4 \tau(1-2 \tau)} .
$$

This leads to $\Delta Q=0$ and

$$
\Delta p_{d}=q \frac{\tau^{\prime}-2 \tau}{\tau^{\prime}-2 \tau+2 \tau^{2}} \stackrel{\epsilon^{2} \ll 1}{\longrightarrow} \frac{q}{1-2 \tau}-\frac{\epsilon^{2} q}{2(1-2 \tau)} .
$$


The result for the free energy then is

$$
\beta f_{\epsilon}(q)-\beta f_{0}=\frac{1}{16 \tau(1-2 \tau)} \epsilon^{4} q^{2}+\mathcal{O}\left(q^{3}\right) .
$$

The restriction $\epsilon^{2} \ll 1$ is basically unnecessary and we could calculate the free energy in the limit $q \ll \min \left(1, \epsilon^{2}\right)$. However, since the regime of large $\epsilon$ will not contribute to the sample-to-sample-fluctuations, we separate it explicitly at this point, and write $\beta f_{\epsilon}(q)-\beta f_{0}(q)=f(\epsilon) q^{2}$ with $f(\epsilon)=\epsilon^{4} \frac{1}{16 \tau(1-2 \tau)}$ for small $\epsilon$. For large $\epsilon \rightarrow \infty, f(\epsilon)$ is equal to $\frac{1}{2}$, which follows from the free energy in the limit $\epsilon \rightarrow \infty$ (equation (5.39) ) derived previously.

\subsubsection{The overlap distribution $P_{\epsilon}^{0}(q)$}

As it turned out in the calculation of the saddle points in the previous subsection, it was practical to work with $q$ instead of $q_{L}$. For the further procedure, we will keep this variable. The exact connection between the free energy fluctuations and bond chaos, equation (5.2) in the large deviations approximation, becomes $\beta^{2} \Delta F_{N}^{2}=\frac{(N-1)^{2} m^{2} \beta^{4}}{16} \int_{0}^{\infty} d \epsilon f_{2}(\epsilon)\left(\left[q^{4}\right]_{0}-\left[q^{2}\right]_{0}^{2}\right)+$ $\frac{(N-1) m \beta^{2}}{4} \int_{0}^{\infty} d \epsilon g_{2}(\epsilon)\left[q^{2}\right]_{0}$, where we used

$$
\left[\left(q_{L}^{13}\right)^{n}\right]_{0}=m^{n} \int d q q^{2 n} P_{\epsilon}^{0}(q)=: m^{n}\left[q^{2 n}\right]_{0} .
$$

The probability distribution $P_{\epsilon}^{0}(q) \sim e^{-N m \beta\left(f_{\epsilon}(q)-f_{0}(q)\right)}$ in the low temperature phase separates into four parts, depending on the range of $\epsilon$. For $\epsilon \ll N^{-1 / 4}$ the contribution to the probability distribution of $q$ of both equations (5.41) and (5.45) (both with $\left.f_{\epsilon}(q) \sim \epsilon^{4} q^{2}\right)$ is negligible. Therefore it is approximately a constant in that range for all $q \in\left[0, q_{E A}\right]$, which follows from the solution for $\epsilon=0$ below $T_{c}$. Equation (5.35) implies that $P_{\epsilon}^{0}(q)$ has an exponentially decaying tail for $q>q_{E A}$ with $e^{-N m c_{0}\left(q-q_{E A}\right)^{3}}$. We define a function $\Theta\left(q-q_{E A}\right)$ which combines both properties. As already pointed out, instead of this plateau in $P_{\epsilon}^{0}(q)$ there should be a $\delta$ peak at $q=q_{E A}$ which we do not see in our calculation. This is due to the fact that we have neglected finite size corrections to the free energy which are dominating in the regime of small $\epsilon$ and which we will implement in the next subsection.

Both solutions we found perturbatively in equation (5.41) and equation 5.45 produce a probability distribution of the form $e^{-N m \beta c_{x} \epsilon^{4} q^{2}}$ with different constants $c_{x}$, but hold in different ranges of $\epsilon$, depending on the relation of $\epsilon^{2}$ and $q$. The order of $\epsilon$ determining the crossover from one regime to the other is where $\epsilon^{2}$ is of the same order as $q$ and $N \epsilon^{4} q^{2}$ (in the range $\left.N^{-1 / 4} \ll \epsilon \ll \epsilon_{0}\right)$ is of order one such as to be the dominating part of the free 
energy. This leads to $\epsilon \sim N^{-1 / 8}$ as the crossover value. For even larger $\epsilon$, we have $e^{-N m f(\epsilon) q^{2}}$, where we introduce a finite cut-off value $\epsilon_{0}$ (independent of $N)$, above which we will be able to neglect the fluctuations of the free energy, as will become clear below. Thus we get the final result

$$
P_{\epsilon}^{0}(q) \propto \begin{cases}\Theta\left(q-q_{E A}\right) & \epsilon \ll N^{-1 / 4} \\ e^{-N m c_{1} \epsilon^{4} q^{2}} & N^{-1 / 4} \ll \epsilon \ll N^{-1 / 8} \\ e^{-N m c_{2} \epsilon^{4} q^{2}} & N^{-1 / 8} \ll \epsilon \ll \epsilon_{0} \\ e^{-N m f(\epsilon) q^{2}} & \epsilon_{0}<\epsilon,\end{cases}
$$

with $c_{1}=\frac{2 \tau+1}{16 \tau}$ and $c_{2}=\frac{1}{16 \tau(1-2 \tau)}$.

\subsubsection{The overlap distribution at $\epsilon=0$}

The probability distribution $P_{\epsilon}^{0}(q)$ from equation (5.47) is the large deviations approximation to the true distribution $P_{\epsilon}(q)$. For small $\epsilon$ it does not exhibit the $\delta$-peak at $q=q_{E A}$, which is the dominating part of the distribution for $\epsilon=0$ (due to replica symmetry). It is generated from the finite size corretions to the free energy, $\Delta F_{\epsilon}$ (which scale with a subdominant power of the system size), and its dominance for small $\epsilon$ reaches up to a crossover value where the large deviations approximation takes over. Unfortunately, the finite size corrections to the free energy are impossible to calculate, but at least we know from Ref. [48] that they grow as $N^{1-y}$ at $\epsilon=0$, with $y=2 / 5$, see also chapter 3 (to be precise, it is the scaling of the ground state energy we have found there, but we expect it to hold for the free energy as well). We assume that this scaling is independent of the value of $\epsilon$, at least for small $\epsilon$. We then find that the large deviations result from equation (5.47) dominates the $\delta$-peak at $q=q_{E A}$ as long as $N m \beta\left(f_{\epsilon}\left(q_{E A}\right)-f_{0}\right) \gg N^{3 / 5}$. Since $q_{E A}=\mathcal{O}(1)$, it follows that this is the case when $N^{3 / 5} \ll N \epsilon^{4}$ (as we have found $P_{\epsilon}^{0}(q) \propto e^{-N m c_{x} \epsilon^{4} q^{2}}$ in both regimes for $\epsilon$ in between $N^{-1 / 4}$ and $\left.\epsilon_{0}\right)$ or $N^{-1 / 10} \ll \epsilon$. We then have

$$
P_{\epsilon}(q) \propto \begin{cases}\delta_{F S}\left(q-q_{E A}\right) & \epsilon \ll N^{-1 / 10} \\ e^{-N c_{2} \epsilon^{4} q^{2}} & N^{-1 / 10} \ll \epsilon \ll \epsilon_{0} \\ e^{-N f(\epsilon) q^{2}} & \epsilon_{0}<\epsilon .\end{cases}
$$

The function $\delta_{F S}\left(q-q_{E A}\right)$ denotes the finite size scaling properties of the $\delta$ peak at $q_{E A}$. The new regime completely replaces the first two regimes we calculated in equation (5.47). For the regime $\epsilon \ll N^{-1 / 4}$, which we deduced from the large deviations result at $\epsilon=0$, this can be well understood, as the plateau we found there doesn't have a physical interpretation. 


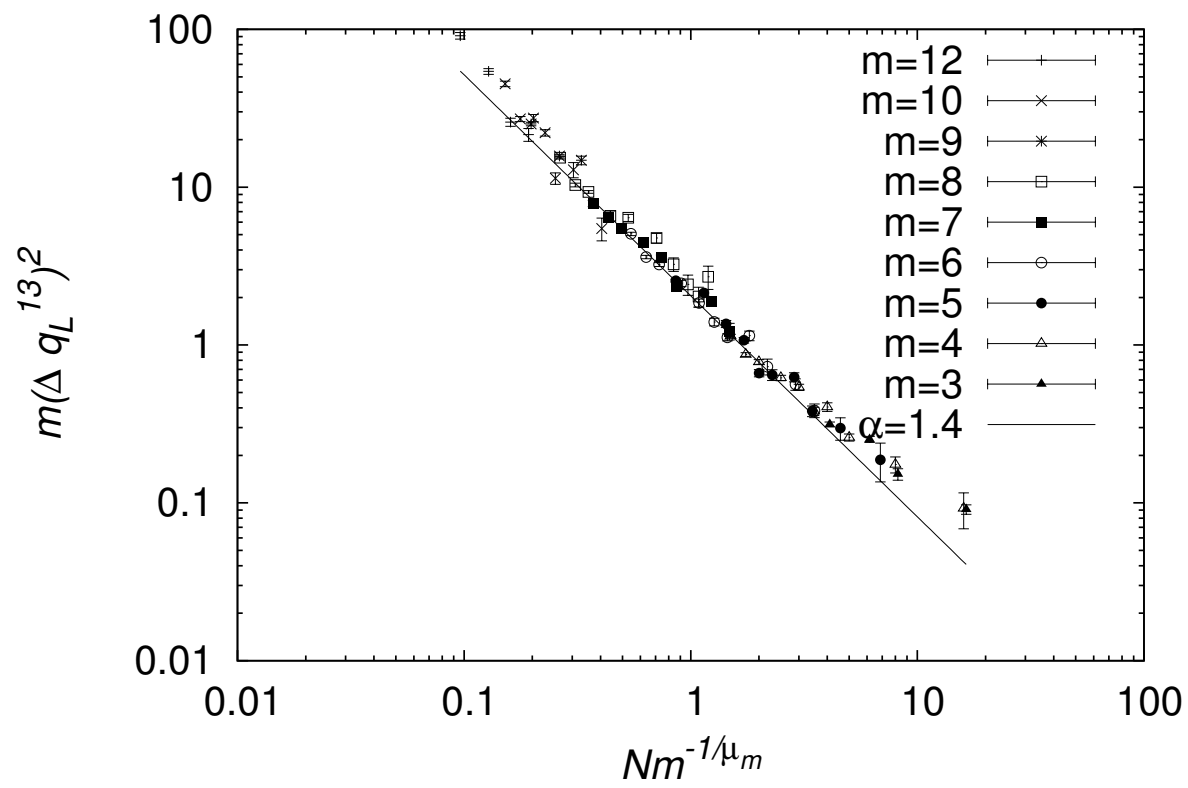

Figure 5.1: Finite size scaling plot of the variance of the link overlap at temperature $T=0.6$. See text for explanation.

In order to calculate the free energy fluctuations with the exact connection to bond chaos, we need the expressions $\left[\left(q_{L}^{13}\right)^{2}\right]-\left[q_{L}^{13}\right]^{2}$ and $\left[q_{L}^{13}\right]$, resp. $\left[q^{4}\right]-$ $\left[q^{2}\right]^{2}$ and $\left[q^{2}\right]$. In the regime $N^{-1 / 10} \ll \epsilon \ll \epsilon_{0}$ we have an explicit expression at hand to calculate them via $\int d q q^{2 n} P_{\epsilon}^{0}(q)$ (with $n=1,2$ ). The last regime in equation (5.48) can be neglected in the next section 5.4, which leaves us with the first regime, the finite size scaling of the $\delta$-peak. For this regime, we resort to a finite size scaling ansatz of the form

$$
\frac{1}{m^{2}}\left(\left[\left(q_{L}^{13}\right)^{2}\right]-\left[q_{L}^{13}\right]^{2}\right)=N^{-\alpha} \mathcal{F}_{m}\left(N^{\beta} \epsilon\right)
$$

for $\epsilon \ll N^{-1 / 10}$ and with a scaling function $\mathcal{F}_{m}(x)$, with $\mathcal{F}_{m}(0)=$ const.. Similarly, we assume that in this regime $\left[q_{L}^{13}\right]$ can be written as

$$
\frac{1}{m}\left[q_{L}^{13}\right]=\mathcal{G}_{m}\left(N^{\rho} \epsilon\right)
$$

with an exponent $\rho$ and a scaling function $\mathcal{G}_{m}(x)$. These scaling functions and exponents will enter the calculation of the fluctuations below. In the following, we will discuss the properties of the scaling functions and support their introduction by numerical investigations from the parallel tempering 
Monte Carlo simulation presented in chapters 2 and 4 by repeating the line of argument that led to figure 4.6.

Due to replica symmetry in the large- $m$ limit we are considering in this chapter, the link overlap distribution in the thermodynamic limit consists of a single $\delta$ peak. Therefore, the variance of this distribution for finite system sizes, which corresponds to the left hand side of equation (5.49), must vanish with the system size, as $N^{-\alpha}$. So far $\alpha$ is unknown, but we can adapt the parallel tempering simulation of the chapters 2 and 4 for the $m$-component spin glass with finite connectivity to this case. There, we were able to measure mean and variance of the distribution of the link overlap. The only adjustment here is to link each spin with each other, instead of using the Bethe lattice of finite connectivity. Again, we have to take the breaking of replica symmetry for small component numbers into account. As we have seen in chapter 4 , there is a critical component number $m_{\text {eff }}(T)$, above which the system is independent of the number of components and thus replica symmetry holds (the large- $m$ limit), while it is broken below. This means, that we have to take care to deduce the finite size scaling of the variance of the link overlap, i.e. the scaling exponent $\alpha$, from the replica symmetric regime. As the critical number of spin components $m_{\text {eff }}(T)$ scales with the system size as $m_{0} \sim N^{\mu_{m}}$ (with $\mu_{m}=2 / 5$ for the SK-model) at $T=0$ [45, 46], we conjectured in chapter 3 that this scaling holds throughout the low temperature phase, see equation (3.6) as $m_{\mathrm{eff}}(T) \sim N^{\mu_{m}}$. If the number of components $m$ that is initially available is larger than $m_{\text {eff }}(T)$ we expect replica symmetry, and for $m<m_{\text {eff }}(T)$ broken replica symmetry. When plotting the variance $\left(\Delta q_{L}^{13}\right)^{2}$ of $q_{L}^{13}$ against the rescaled variable $x=$ $N m^{-1 / \mu_{m}}$, we should find the replica symmetric regime for small $x$, whereas for larger $x$ the variance should cross over to a constant variance, which is a property of broken replica symmetry. This is precisely what we observe in figure 5.1, although the crossover is so slow that we do not see the expected plateau yet.

The parallel tempering Monte Carlo algorithm is described in chapter 2 . For maximally available component numbers from 3 to 12 , we use system sizes from $N=64$ up to $N=256$ in the temperature range of $[0.6,1.6]$ here. In that way we produced for two different replicas (with the same set of coupling constants since we are considering $\epsilon=0$ ) at least 64 statistically independent sets of spin configuration for every temperature. From these, we calculated the variance of the link overlap $\left(\Delta q_{L}^{13}\right)^{2}=\left[\left(q_{L}^{13}\right)^{2}\right]-\left[q_{L}^{13}\right]^{2}$ at $T=0.6$. In order not to have a different estimate for $\alpha$ from every single maximal number of components $m$, we rescale the $x$-axis as described above to find the universal scaling of $\left(\Delta q_{L}^{13}\right)^{2}$. Therefore after rescaling the system size $N$ with $m^{-1 / \mu_{m}}$, we have to scale the $y$-axis $\left(\Delta q_{L}^{13}\right)^{2}$ with the appropriate 
power of $m$ to make the data collapse to a single scaling function. This scaling function then is independent of $m$ and we can deduce the universal scaling with the system size as $N^{-\alpha}$ in the replica symmetric region. Independence of $m$ is achieved by scaling $\left(\Delta q_{L}^{13}\right)^{2}$ with $m$. Figure 5.1 shows the results for the various component numbers. As in chapter 4 , for smaller component numbers, it is possible to get to larger system sizes, since less temperatures are needed for the parallel tempering algorithm.

According to the scaling ansatz above, we have $m^{-2}\left(\Delta q_{L}^{13}\right)^{2} \sim\left(N m^{-1 / \mu_{m}}\right)^{-\alpha} m^{-\alpha / \mu_{m}}$ at $\epsilon=0$. This can be rewritten as $m^{\alpha / \mu_{m}-2}\left(\Delta q_{L}^{13}\right)^{2} \sim\left(N m^{-1 / \mu_{m}}\right)^{-\alpha}$, which is the expression we compare to the numerical results. The data in Fig. 5.1 show on the one hand that $\alpha / \mu_{m}-2 \approx 1$ (which can be deduced from the proper scaling of the $y$-axis as $\left.m\left(\Delta q_{L}^{13}\right)^{2}\right)$ and on the other hand that $\alpha \approx 1.4$, which we find for small $x$. The observation $\alpha / \mu_{m}-2 \approx 1$ implies $\alpha \approx \frac{6}{5}$. Together with the second, more direct, observation of $\alpha$, the data show that $\alpha \geq \frac{6}{5}$, and as we will see below, this is all we need to know.

The scaling exponents $\beta$ and $\rho$ are unknown, too. However, we let the scaling functions of this subsection cross over smoothly to the large deviations approximation of the link overlap distribution, where we have explicit expressions at hand to calculate $\left[\left(q_{L}^{13}\right)^{2}\right]-\left[q_{L}^{13}\right]^{2}$ and $\left[q_{L}^{13}\right]$, i.e. for $\epsilon \gg N^{-1 / 10}$ (to this end it is necessary to make the scaling functions $\mathcal{F}$ and $\mathcal{G}$ depend on $m$, denoted by the index $m$ in the equations (5.49) and (5.50)). This reveals scaling relations in the next section, which make the explicit values of the exponents of $\beta$ and $\rho$ irrelevant for the calculation of the free energy fluctuations.

\subsection{Calculation of the sample-to-sample Fluc- tuations}

It remains to calculate $\left[q^{n}\right]_{0}$ for $N^{-1 / 10} \ll \epsilon \ll \epsilon_{0}$, insert it into the equations (5.2) and (5.11) and adjust the scaling ansatz of the previous section in such a way to connect both regimes properly. We apply the saddle point method to write for $\epsilon$ with $N^{-1 / 10} \ll \epsilon \ll \epsilon_{0}$ (with $P_{\epsilon}^{0}(q)=\frac{1}{\mathcal{N}_{q}} e^{-\beta N m f_{\epsilon}(q)}$ and the normalization constant $\mathcal{N}_{q}$ )

$$
\left[q^{n}\right]_{0}=\frac{1}{\mathcal{N}_{q}} \int_{0}^{\infty} d q q^{n} e^{-N m c_{2} \epsilon^{4} q^{2}+\mathcal{O}\left(q^{4}\right)}
$$

Here, we set the upper bound from 1 to $\infty$, which introduces only exponentially small errors. Further, we neglect the term of order $q^{4}$ in the exponent, 
and get $\left(\right.$ with $\left.\mathcal{N}_{q}=\frac{\Gamma\left(\frac{1}{2}\right)}{2 \sqrt{N m c_{2} \epsilon^{4}}}\right)$

$$
\begin{aligned}
{\left[q^{n}\right]_{0} } & =\frac{1}{\Gamma\left(\frac{1}{2}\right)} \int_{0}^{\infty} d x x^{\frac{n-1}{2}}\left(N m c_{2} \epsilon^{4}\right)^{-n / 2} e^{-x} \\
& =\frac{\left(N m c_{2} \epsilon^{4}\right)^{-n / 2}}{\Gamma\left(\frac{1}{2}\right)} \Gamma\left(\frac{n+1}{2}\right) .
\end{aligned}
$$

This finally yields

$$
\begin{aligned}
{\left[q^{2}\right]_{0} } & =\frac{1}{2 N m c_{2} \epsilon^{4}} \\
{\left[q^{4}\right]_{0} } & =\frac{3}{\left(2 N m c_{2} \epsilon^{4}\right)^{2}} .
\end{aligned}
$$

Now, we can calculate the sample-to-sample-fluctuations through equations (5.2) and (5.11) by taking the leading order in $N$ of every integral into account. The first integral, $I_{21}$, (with $f_{2}(\epsilon)=2 \epsilon^{3}+\mathcal{O}\left(\epsilon^{5}\right)$ for small $\epsilon$ ) separates into three integration intervals corresponding to the three regimes of the probability distribution $P_{\epsilon}(q)$. We neglect the range of $\epsilon>\epsilon_{0}$, because it gives a contribution of order 1 which we are not interested in. For the part $\epsilon \ll N^{-1 / 10}$ we have the scaling ansatz for $\left[\left(q_{L}^{13}\right)^{2}\right]-\left[q_{L}^{13}\right]^{2}=m^{2}\left(\left[q^{4}\right]-\right.$ $\left.\left[q^{2}\right]^{2}\right)=m^{2} N^{-\alpha} \mathcal{F}_{m}\left(N^{\beta} \epsilon\right)$. This ansatz must match $m^{2}\left(\left[q^{4}\right]_{0}-\left[q^{2}\right]_{0}^{2}\right)$ from the neighbouring regime (the large deviations regime) at the crossover point $\epsilon=N^{-1 / 10}$. With this restriction we force the small deviations and the large deviations approximations to describe a single probability distribution $P_{\epsilon}(q)$. At the crossover point, in the large deviations regime, it goes as $N^{-2} \epsilon^{-8} \sim N^{-6 / 5}$ (see equations (5.53) and (5.54)). For the other regime, we let the scaling function $\mathcal{F}_{m}(x)$ decay as $x^{-\gamma} / m^{2}$ for $x \rightarrow \infty$ with an exponent $\gamma$. Inserting this ansatz, we have $m^{2}\left(\left[q^{4}\right]_{0}-\left[q^{2}\right]_{0}^{2}\right)=N^{-\alpha}\left(N^{\beta} N^{-1 / 10}\right)^{-\gamma}$ at the crossover point and the exponent $\gamma$ obeys $\gamma\left(\beta-\frac{1}{10}\right)=\frac{6}{5}-\alpha$, in order to match the scaling of $N^{-6 / 5}$ from the neighbouring regime.

For the integral $I_{21}$, this leads to

$$
\begin{aligned}
I_{21} & =\int_{0}^{N^{-1 / 10}} d \epsilon 2 \epsilon^{3} N^{-\alpha} \mathcal{F}_{m}\left(N^{\beta} \epsilon\right)+\int_{N^{-1 / 10}}^{\epsilon_{0}} d \epsilon 2 \epsilon^{3} \frac{1}{2\left(N c_{2} \epsilon^{4}\right)^{2}} \\
& \sim 2 N^{-\alpha-4 \beta} \int_{0}^{N^{\beta-1 / 10}} d x \mathcal{F}_{m}(x) x^{3}+N^{-2} \int_{N^{-1 / 10}}^{\epsilon_{0}} d \epsilon \frac{\epsilon^{-5}}{c_{2}^{2}}+\ldots \\
& \sim \text { const. }_{.} N^{-\alpha-\frac{4}{\gamma}\left(\frac{6}{5}-\alpha\right)-\frac{2}{5}}+\text { const. }_{2} N^{-8 / 5}
\end{aligned}
$$

as long as $\gamma>4$. If $\gamma<4$, this part of the free energy fluctuations is of order $\mathcal{O}\left(N^{-8 / 5}\right)$. Together with the leading prefactor $N^{2}$ of the first integral in equation 5.2 , we find that $\Delta F_{N}^{2}$ scales at least as $N^{2 / 5}$. 
We still have to estimate the leading scaling behaviour of the second integral of equation (5.2). For this purpose we apply the scaling ansatz $\frac{1}{m}\left[q_{L}^{13}\right]=\mathcal{G}_{m}\left(N^{\rho} \epsilon\right)$. The scaling function $\mathcal{G}_{m}(x)$ should have the properties $\mathcal{G}_{m}(x) \stackrel{x \rightarrow \infty}{\rightarrow} x^{-\eta} / m$ and $\mathcal{G}_{m}(x) \stackrel{x \rightarrow 0}{\rightarrow}$ const. and should scale as $N^{-1} \epsilon^{-4}$ for $\epsilon=N^{-\frac{1}{10}}$ in order to match the neighbouring regime, similar to above. This yields the scaling relation $\rho=\frac{3}{5 \eta}+\frac{1}{10}$ and contributes to the free energy fluctuations as (with $g_{2}(\epsilon)=\epsilon^{3}+\mathcal{O}\left(\epsilon^{5}\right)$ for small $\epsilon$ )

$$
\begin{aligned}
I_{22} & =\int_{0}^{N^{-1 / 10}} d \epsilon \epsilon^{3} \mathcal{G}_{m}\left(N^{\rho} \epsilon\right)+\int_{N^{-1 / 10}}^{\epsilon_{0}} d \epsilon \frac{\epsilon^{3}}{2 N c_{2} \epsilon^{4}}+\ldots \\
& =\text { const. }{ }_{3} N^{-\frac{12}{5 \eta}-\frac{2}{5}}+\text { const. } . \frac{\log N}{N},
\end{aligned}
$$

provided that $\eta>4$. If $\eta<4$ the first part of the integral is $\mathcal{O}\left(N^{-1}\right)$ instead. In the second route to chaos, $\beta^{2} \Delta F_{N}^{2}=\frac{(N-1)^{2} \beta^{4}}{16} I_{21}+\frac{(N-1) \beta^{2}}{4} I_{22}$, this integral has the leading prefactor $N$. Hence, the scaling exponent of the leading contribution of $\Delta F_{N}^{2}$ from this part is $\frac{3}{5}-\frac{12}{5 \max (\eta, 4)}$. Together with equation (5.55) we find the leading scaling exponent of the sample-to-samplefluctuations with the system size, $\Delta F_{N} \sim N^{\mu}$, to be

$$
\mu=\max \left[\frac{1}{5}, \frac{4}{5}-\frac{\alpha}{2}-\frac{2}{\max (\gamma, 4)}\left(\frac{6}{5}-\alpha\right), \frac{3}{10}-\frac{6}{5 \max (\eta, 4)}\right] .
$$

This equation looks not very promising at first. However, we can further restrict the range of possible values of $\mu$. The second part can be written as $\frac{1}{5}+\left(\frac{6}{5}-\alpha\right)\left(\frac{1}{2}-\frac{2}{\max (\gamma, 4)}\right)$. We see that it is $\leq \frac{1}{5}$ for $\alpha \geq \frac{6}{5}$, which we found numerically in the previous section. The third part is at most equal to $\frac{3}{10}$, for any finite value of $\eta$ it is smaller. Hence, we finally find $\frac{1}{5} \leq \mu \leq \frac{3}{10}$.

Further limitation of the exponents we use can be deduced from the first route to chaos. As described in section 5.1, it is similar to the second route such that we can apply the same scaling ansätze as above. However, there are two major differences. The first difference are the functions $f_{1}(\epsilon)$ and $g_{1}(\epsilon)$ (instead of $f_{2}(\epsilon)$ and $g_{2}(\epsilon)$ ), which can be approximated by $2 \pi \epsilon^{2}+\mathcal{O}\left(\epsilon^{4}\right)$, resp. $\pi+\mathcal{O}\left(\epsilon^{2}\right)$. The second difference is the minus sign in front of $I_{11}$. The integral itself is positive. However, as the fluctuations can not be negative, the leading order of $-\frac{(N-1)^{2} \beta^{4}}{16} I_{11}$ must be compensated by the leading order of the second part $\frac{(N-1) \beta^{2}}{4} I_{12}$. The calculations follow those from the second route to chaos. The contribution from $I_{11}$ is

$I_{11}=m^{2} \int_{0}^{\epsilon_{0}} d \epsilon f_{1}(\epsilon)\left(\left[q^{4}\right]-\left[q^{2}\right]^{2}\right) \sim$ const. ${ }_{5} N^{-\alpha-\frac{3}{\gamma}\left(\frac{6}{5}-\alpha\right)-\frac{3}{10}}+$ const. ${ }_{6} N^{-3 / 2}+\ldots$ 


\subsection{CALCULATE THE SAMPLE-TO-SAMPLE FLUCTUATIONS}

From $I_{12}$ we get

$$
I_{12}=m \int_{0}^{\epsilon_{0}} d \epsilon g_{1}(\epsilon)\left[q^{2}\right] \sim \text { const. } .7 N^{-\frac{3}{5 \eta}-\frac{1}{10}}+\text { const. }{ }_{8} N^{-7 / 10} .
$$

Comparing the leading orders of both integrals (and taking their leading prefactors $N^{2}$ and $N$ into account), we find that $\frac{9}{10}-\frac{3}{5 \eta}$ must be equal to $\max \left[\frac{17}{10}-\alpha-\frac{3}{\gamma}\left(\frac{6}{5}-\alpha\right), \frac{1}{2}\right]$ (the second part of $N I_{12}$ is definitely subdominant, since it can not cancel the leading term of $N^{2} I_{11}$, which is at least $N^{1 / 2}$ ). Hence $\eta$ is equal to or larger than $\frac{3}{2}$. This limitation shows no contradiction to the result of $\frac{1}{5} \leq \mu \leq \frac{3}{10}$ we found from the second route to chaos.

In summary, we find the sample-to-sample fluctuations of the free energy $F_{N}$ to scale with the system size as $N^{\mu}$, with $\frac{1}{5} \leq \mu \leq \frac{3}{10}$. The result is given in equation (5.57). There, we have found three different contributions to the scaling of the free energy fluctuations of which we are interested in the largest one. From a numerical investigation, using the Monte Carlo parallel tempering algorithm, we found $\alpha \geq \frac{6}{5}$ and can therefore neglect the second contribution. Even more, $\mu>\frac{1}{5}$ is only possible if $\eta>12$ (for $\eta \leq 12$ the third term on the right hand side of equation (5.57) is at most equal to $\frac{1}{5}$ ), which seems not very likely. In any case, the fluctuations are far from being trivial as they do not fall into any of the four known universality classes of extreme value statistics described in the introduction.

The result of this chapter was derived involving a variety of mathematical technicalities. However, it is based on a few assumptions. As the technicalities shall not prevent the reader from understanding the main assumptions leading to the result $\frac{1}{5} \leq \mu \leq \frac{3}{10}$, we repeat them in the following. First of all, we have adapted the exact connection between bond chaos and the sampleto-sample fluctuations of the free energy to the case of the $m$-component spin glass in the large- $m$ limit. This involved solely mathematically exact manipulations and the derivation can therefore not be contested. Second, bond chaos is calculated via a large deviations approximation (with the help of the replica method) for the free energy of two systems, constrained to have a certain link overlap. We saw that this approximation can not hold for small deviations, which we are not able to calculate directly. Third, we had to implement these small deviations for the region of small $\epsilon$. For this purpose, we resorted to the earlier result of the scaling of the small deviations for $\epsilon=0$ from Ref. [48], and deduced from it a crossover value of $\epsilon$ below which the small deviations dominate the probability distribution $P_{\epsilon}\left(q_{L}\right)$, while above the large deviations dominate. The small deviations regime was then covered by a scaling ansatz, which involves three different exponents. Assuming this ansatz to cross over smoothly to the large deviations regime, we had to 
introduce two additional scaling exponents. Supported by numerical results, we found $\mu$ to depend on only three of the five exponents. Finally, we could limit $\mu$ to the range of $\frac{1}{5} \leq \mu \leq \frac{3}{10}$, with $\mu>\frac{1}{5}$ only for $\eta>12$, which makes $\mu$ exactly equal to $\frac{1}{5}$ very likely. Nevertheless, to ensure $\mu=\frac{1}{5}$, it would be necessary to measure $\eta$, which should be feasible.

It should be mentioned that the exponent $\mu=\frac{1}{5}$ was earlier suggested in Ref. [45]. However, this derivation was footed on a wrong assumption, which was a gap in the eigenvalue spectrum of the inverse susceptibility matrix. This gap was later shown not to exist in Ref. [46]. The derivation in this chapter is completely different from the one in Ref. [45].

How does the result of $\mu=\frac{1}{5}$ relate to other spin glass models? First of all, the sample-to-sample fluctuations in the $m$-component model differ from those of the spherical model, which follows the Tracy-Widom distribution as $N^{1 / 3}$. A single universality class of replica symmetric mean-field spin glass models is therefore ruled out, and the difference of both models in the low temperature phase is further substantiated (though their free energies are equal in the thermodynamic limit [38, 41]). Furthermore, the fluctuations probably differ from those of the Ising spin glass as well, as they are favoured to scale as $N^{1 / 6}$. If they scaled as $N^{1 / 4}$, which is the alternatively favoured scaling behaviour, equal scaling of the $m$-component spin glass demands $\eta=24$, which is far from being likely. Hence, our computation of $\mu$ proves the $m$-component spin glass to be an interesting spin glass model in its own rights. 


\section{Chapter 6}

\section{Conclusion}

What have we learned after all? We can certainly say that the $m$-component spin glass shows a variety of astonishing and unique properties, which makes it a valuable member of the spin glass family. To begin with, it was shown how to apply or adjust succesful methods of statistical physics to the case of the $m$-component spin glass, like the Cavity method, the parallel tempering Monte Carlo algorithm, an analytical investigation of spin glasses on Bethe lattices reminiscent of the Cavity method and the calculation of bond chaos and the sample-to-sample fluctuations of the free energy.

Making use of the Cavity method by a self-consistent effective-field approach, we observed a phase transition which is as odd as interesting. It will be very exciting to see whether these results can contribute to a general picture describing the low temperature phase of vector spin glasses, admitting that most of the results are restricted to the vicinity of the phase transition. The common picture of the phase transition of the $m$-component spin glass on a Bethe lattice is a transition from a paramagnetic phase (with a value of zero of each of the different order parameters we have employed) into a low temperature phase (with a non-zero value of each of the different order parameters), for which there seems to be no freezing in time in contrast to the usual picture of spin glass phase transitions. Instead we find a dynamical configuration of spins, resp. of the local fields of the spins, which is subject to rotations in thermal equilibrium (explicitly not due to thermal fluctuations). As expected, the transition is accompanied by an unusual phase transition, the generalized Bose-Einstein condensation (for which the local fields use only a fraction of the available $m$ components and this fraction scales with the system size as $N^{\mu_{m}}$ ). However, there is an interesting temperature dependence of the effective number of components $m_{\mathrm{eff}}(T)$, which we find to be closely related to the rotations of the configuration of local fields. This relation stems from the fact that at the critical temperature there is either 
a one-dimensional configuration of local fields (which is frozen in time) or a two-dimensional configuration which rotates in the twodimensional plane it spans. For lower temperatures there occur more and more of these rotations resulting in a complex dynamical nature of the low temperature phase. It must be emphasized that it is the simulation of single Bethe lattices (developed in this thesis) which allows us to get rid of thermal fluctuations which usually prevent detecting such underlying mechanisms of the phase transition. A further result of this approach is the stability of replica symmetry in the large- $m$ limit.

In addition to these numerical results we have been able to confirm the stability of replica symmetry analytically in section 4.3 . There we presented a method how to expand the free energy of the $m$-component spin glass in terms of overlaps $q_{\alpha \beta}$ around the saddle point of the fully connected model with $k, m \rightarrow \infty$, in both a power series in $\frac{1}{k}$ and $\frac{1}{m}$. Furthermore we calculated the sample-to-sample fluctuations of the free energy of the fully connected version of our model and found that they are different from other prominent spin glass models and do not fall into any of the known universality classes of extreme value statistics. The final result for the scaling of the sample-tosample fluctuations is $F_{N} \sim N^{\mu}$ with $\frac{1}{5} \leq \mu \leq \frac{3}{10}$ and probably $\mu=\frac{1}{5}$. This scaling is different from finitely connected models which obey $F_{N} \sim N^{1 / 2}$ and from the spherical and the Ising spin glass. Even more, the fully connected $m$-component spin glass is not a member of any known universality class of extreme value statistics, see the discussion at the end of chapter 5 .

The general picture of the phase transition is completed by two additional observations. On the one hand, the spin-spin-correlations in the low temperature phase scale with the system size in a power law dependence with the scaling exponent $\mu_{m}$, which initially was found within the generalized Bose-Einstein phase transition. This property is reminiscent of the Kosterlitz-Thouless transition of the XY ferromagnet (for which there is no long range order and a vanishing order parameter), though we can define the order parameter of the $m$-component spin glass in such a way to make it finite in the thermodynamic limit (without loosing the scaling property). On the other hand we observe replica symmetry breaking if the effective number of components is found to be larger than the maximally available number of components $m$. To explain it the other way round, there is a large- $m$ limit for finite system sizes as long as the maximal number of components is larger than $m_{\text {eff }}(T)$, for which replica symmetry holds. In particular, replica symmetry breaking happens when the complex superposition of rotations needs more than the available $m$ components. Then, the displayed behaviour in $m$ dimensions is only the projection onto the $m$ dimensional space of more complex underlying dynamics, which happen in a higher dimensional space 
(and would be replica symmetric in that space). Unfortunately, we have not been able to confirm this picture of replica symmetry breaking by the analytical approach in section 4.3 , possibly because there is no replica symmetry breaking for an analytical continuation in a power series in $\frac{1}{m}$ around the $m \rightarrow \infty$-limit, or it does occur only for advanced breaking schemes such as 2-RSB or full RSB.

Is it possible to relate our findings to results from other publications? So far, the phase transition of the vector spin glass in the large- $m$ limit has not been intensively investigated. However, a phase transition of this model is observed on low dimensional lattices and for the fully connected SK model, accompanied by the power law scaling of the order parameter as $N^{-\mu_{m}}$ consistent with our results [47]. Replica symmetry breaking is generally expected to happen similarly to the scenario presented here (i.e. for $\left.m<m_{\mathrm{eff}}(T)\right)$, though the temperature dependence of $m_{\mathrm{eff}}(T)$ has so far not been observed [41, 45, 46, 47]. In fact, the technique of iterating the configuration of mean fields is the key to these results as it overcomes the influence of thermal fluctuations, which are usually still large enough to obscure the underlying nature of the transition in the vicinity of $T_{c}$. The observation of a very low number of effective components at the critical temperature relates the $m$-component spin glass closely to XY- and Heisenberg spin glasses, which have been investigated in low dimensions [32, 33, 34, 35, 90, 91. In Ref. [90, 91] it has been reported that the ground state of the XY spin glass on a square lattice with bimodally distributed bonds is unique up to the global rotation of all spins, similar to the observation of a rotating configuration of fields in this thesis (although the inherent dynamic implied by the results of this thesis has not been reported). The main question of interest in all of these publications was whether there is a spin glass phase transition at a finite temperature and whether there is a chiral glass transition decoupled from the spin glass transition. In this thesis we have not investigated a chiral glass transition, which is not directly applicable due to the inhomogeneous structure of Bethe lattices compared to square and cubic lattices. Therefore we can not contribute to the question of a spin-chirality decoupling. Even more, analysing configurations of effective fields on single low dimensional lattices exhibits a critical temperature far above $T_{c}$ found in the cited publications (we have done such a simulation, though the Cavity method is obviously not really appropriate for these low dimensional lattices as it is based on the assumption of large loops (resp. a locally tree like lattice structure); In low dimensional lattices, loops are small and do not scale with the system size). However, we suggest a possible relation between chiralities and the observed rotations in thermal equilibrium. For this purpose, we notice that the Kosterlitz-Thouless transition of the unfrustrated XY ferro- 
magnet (which we have found the phase transition of the $m$-component spin glass to be reminiscent of) is explained by the occurence of vortices, which are singular spin configurations in which one sums up a total angle of multiples of $2 \pi$ when going around a closed path containing the centre of the vortex, see figure 6.1. The vortices are bound in pairs in the low temperature phase, whereas they become unbounded above the phase transition. Without going into detail, it is this binding which is responsible for the phase transition [49, 53. In case of the frustrated XY spin glass, one sums up an angle of $2 \pi$ when going around an unfrustrated plaquette, whereas the total angle is $\pi$ in case of a frustrated plaquette [5]. This is precisely the basic consideration of the chirality picture. Therefore, in case of a frustrated spin system, we find half-vortices, which are expected to determine the physics in the low temperature phase, see Ref. [92] for an analytical description of the XY spin glass in two dimensions. Though we can not present a similar theory for the $m$-component spin glass, it is not far from being likely that these ordering phenomena result in a rotation of the local fields, possibly due to the inhomogeneous structure of the Bethe lattice concerning the size and distribution of loops. Be that as it may, this open point needs further investigation beyond this thesis.

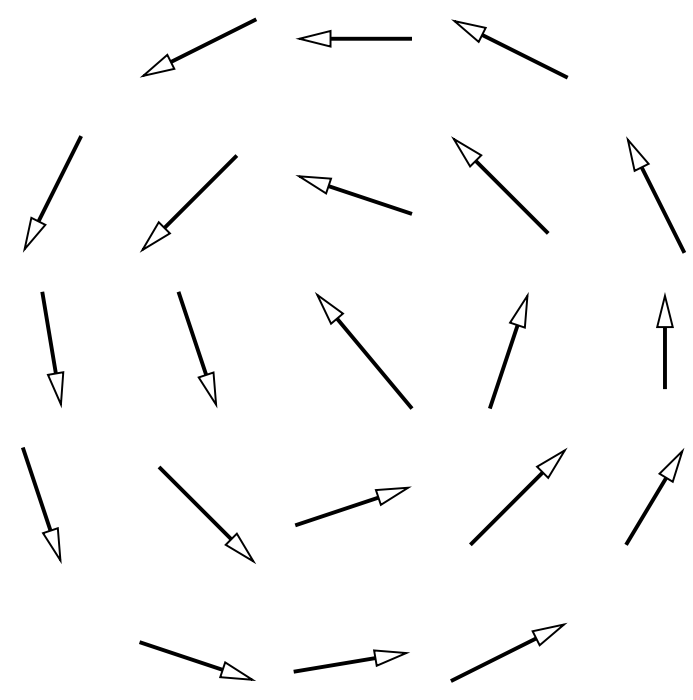

Figure 6.1: Schematic plot of a vortex for which the spins rotate once anticlockwise on travelling anticlockwise around the centre. In the low temperature phase, vortices are bound in pairs.

The manifestation of replica symmetry breaking of the $m$-component spin glass on a Bethe lattice as a complex rotation in a more than $m$ dimensional space is hardly comparable with the "peculiar" replica symmetry breaking of 
the threedimensional Heisenberg spin glass, reported in Ref. [93, 94. Besides the fact that the three-dimensional Heisenberg spin glass is suspicious of exhibiting a one step broken replica symmetric solution, i.e. a scenario of replica symmetry breaking different to the full hierarchical replica symmetry breaking of Parisi, the results found in publications on Heisenberg spin glasses do not allow for an interpretation in the sense of the results of this thesis. The predicted replica symmetric range close to $T_{c}$ has not been reported either.

The analytical investigation of section 4.3 has to be related to the publications of de Almeida et al., Ref. [41], and Viana, Ref. [44, 50]. In Ref. [41] it was shown that replica symmetry is broken in the fully connected model of the $m$-component spin glass for all finite $m$. We have found the $m$-component spin glass to be replica symmetric for $m \rightarrow \infty$, but have not been able to reproduce the breaking of replica symmetry for finite $m$ by expanding around the $m \rightarrow \infty$-limit in a power series in $\frac{1}{m}$. It might be possible that replica symmetry breaking occurs in higher orders or advanced breaking schemes. However, it might also be that it is not possible to continue the free energy from $m \rightarrow \infty$ to finite $m$ for the reason that the method only works for tree like lattices, which requires the limit $N \rightarrow \infty$. Alas, this limit requires $m \rightarrow \infty$ for replica symmetry as the necessary number of components to have a replica symmetric spin glass scales as $N^{\mu_{m}}$, such that the corrections in $m$ vanish for $N \rightarrow \infty$. Therefore it might be useful to extend the calculation by using finite $m$ as in Ref. [41] (and presented partially in appendix B.4) and to compare both starting points.

The situation is different in case of the expansion in $\frac{1}{k}$. There, we find coexistence of replica symmetry and broken replica symmetry up to the order $\frac{1}{k^{2}}$ of the free energy (which involves the overlaps $q_{\alpha \beta}$ up to order $\frac{1}{k}$ ). As already discussed at the end of this section, this discovery needs to be further investigated. On the one hand it is possible that replica symmetry is restored when calculating the overlaps in order $\frac{1}{k^{2}}$ (similar to the expansion in $\frac{1}{m}$ ). On the other hand, if coexistence really holds it gives a new starting point to analyze the breaking of replica symmetry of the $m$-component spin glass for finite connectivities and finite component numbers, regarding the possible phase transition from replica symmetry to broken replica symmetry presented in figure 4.7. Concerning the work of Viana, who initially found the upper critical dimension (above which mean field theory holds, i.e. replica symmetry in this case) and the lower critical dimension (below which Edwards-Anderson order vanishes) are, surprisingly, both equal to eight [44] (but later found $d_{\text {upper }}=14$ and suggested that it might be even higher, see Ref. [50]), our result suggests the upper critical dimension to be $\infty$. Regarding the lower critical dimension (where the phase transition vanishes), the 
Bethe lattice should be above any $d_{l}$ as its structure is in between the fully connected lattice and finite dimensional lattices.

Regarding different aspects of this thesis (especially the nature of the phase transition), it would be very interesting to go beyond the Bethe lattice approximation and, for instance, to introduce corrections in $\frac{1}{k}$ due to the loops of the lattice which lead to a feedback of a spin onto itself. Nevertheless, in this thesis, the $m$-component spin glass shows new and unique properties and in some aspects allows for more accurate statements compared to the Ising spin glass. Beyond that, this thesis provides some interesting observations and it will be exciting to see whether these can be confirmed in future studies. 


\section{Appendix A}

\section{Self-consistent effective-field approach}

In order to find a relation how to calculate the cavity field $\vec{h}_{0}$ from the cavity fields $\vec{h}_{1} \ldots \vec{h}_{k}$, we have to calculate two spin expectation values, resulting on the one hand from equation (2.6) and on the other hand from the partition function of a spin in a single field $\vec{h}_{0}$. The self-consistent effective field approach assumes that both spin expectation values are equal, i.e. that $\vec{h}_{0}$ produces the same expectation value $\left\langle\vec{s}_{0}\right\rangle_{c}$ (if $\vec{s}_{0}$ is solely subject to this field) as the cavity partition function (2.6). The generalized result of calculating the partition function of a single spin in a single field will be used frequently in this section. Therefore, we take an arbitrary vector field $\tilde{\vec{h}}$ instead of $\vec{h}_{0}$ and calculate the general partition function $\tilde{Z}$ of the spin $\tilde{\vec{s}}$ in this field

$$
\tilde{Z}=\int d^{m} s \delta\left(m-(\tilde{\vec{s}})^{2}\right) \exp \{\beta \tilde{\overrightarrow{\vec{s}}} \tilde{\vec{h}}\}
$$

Using the integral representation of the $\delta$-function with an integration parameter $z$, this becomes a Gaussian integral for $\tilde{\vec{s}}$. We introduce a positive constant $c$ to ensure convergence of this integral and get

$$
\begin{aligned}
\tilde{Z} & =\int_{-i \infty+c}^{i \infty+c} \frac{d z}{2 \pi} \int d^{m} \tilde{s} \exp \left\{\beta \tilde{\vec{h}} \tilde{\vec{s}}+z\left(m-\left(\tilde{\vec{s}}_{i}\right)^{2}\right)\right\} \\
& =\int_{-i \infty+c}^{i \infty+c} \frac{d z}{2 \pi} \exp \left\{\beta^{2} \tilde{\vec{h}}^{2} / 4 z+z m-\frac{m}{2} \ln \frac{z}{\pi}\right\} .
\end{aligned}
$$

We solve this integral by a saddle point approach with the large parameter $m$. To do so, we extract a factor $m$ from the first term in the exponent by substituting $\tilde{\vec{h}} / \sqrt{m} \rightarrow \tilde{\overrightarrow{h^{\prime}}}$ and solve the saddle point equation $\frac{\partial}{\partial z}\left(\beta^{2}\left(\tilde{\vec{h}}^{\prime}\right)^{2} / 4 z+\right.$ 
$\left.z-\frac{1}{2} \ln z\right)=0$ with the relevant solution $z=z^{0}=\frac{1}{4}\left(1+\sqrt{1+4 \beta^{2}\left(\tilde{\vec{h}}^{\prime}\right)^{2}}\right)$. Apart from irrelevant constants, the partition function then is

$$
\tilde{Z}=\exp \left\{m\left(\beta^{2}\left(\tilde{\vec{h}}^{\prime}\right)^{2} / 4 z^{0}+z^{0}-\frac{1}{2} \ln z^{0}\right)\right\} .
$$

We obtain the spin expectation value $\langle\tilde{\vec{s}}\rangle_{c}$ by differentiating $\tilde{Z}$ with respect to $\tilde{\vec{h}}$ (as it is the partition function of the spin $\tilde{\vec{s}}$ in this field)

$$
\langle\tilde{\vec{s}}\rangle_{c}=\frac{1}{\beta} \frac{\partial \ln \tilde{Z}}{\partial \tilde{\vec{h}}}=\frac{\beta \overrightarrow{\vec{h}}}{2 z^{0}}=\frac{2 \beta \tilde{\vec{h}}}{1+\sqrt{1+4 \beta^{2}\left(\tilde{\vec{h}}^{\prime}\right)^{2}}} .
$$

Substituting $\tilde{\vec{s}}$ and $\tilde{\vec{h}}$ back to the specific notation $\vec{s}_{0}$ and $\vec{h}_{0}$, we have found the first spin expectation value $\left\langle\vec{s}_{0}\right\rangle_{c}$ of the spin $\vec{s}_{0}$ in a single field $\vec{h}_{0}$.

To derive the spin expectation value from equation (2.6) resulting from the cavity approach, we have to calculate this partition function. It consists of an integral over $\vec{s}_{0}$ and of the product of the partition functions $Z_{i}$. As these have a similar structure as the partition function $(\mathrm{A} .1)$, which we have just calculated, we can use the result of equation $\sqrt{A .4}$ for the partition functions $Z_{i}$ by repeating this calculation with the substitution $\tilde{\vec{h}} \rightarrow J_{i} \vec{s}_{0}+$ $\vec{h}_{i}$ in equation (A.1). The solution of the saddle point equations for the parameter $z_{i}$ then is $z_{i}^{0}=\frac{1}{4}\left(1+\sqrt{1+4 \beta^{2}\left(J_{i} \overrightarrow{s_{0}^{\prime}}+\overrightarrow{h_{i}^{\prime}}\right)^{2}}\right)$ and the partition function $Z_{0}$ is

$$
Z_{0}=\int d^{m} s_{0} \delta\left(m-{\overrightarrow{s_{0}}}^{2}\right) \exp \left\{m \sum_{i=1}^{k}\left(\beta^{2}\left({\overrightarrow{h_{i}^{\prime}}}^{\prime}+{J_{i}}_{{\overrightarrow{s_{0}}}^{\prime}}\right)^{2} / 4 z_{i}^{0}+z_{i}^{0}-\frac{1}{2} \ln z_{i}^{0}\right)\right\},
$$

with $\overrightarrow{s_{0}}=\overrightarrow{s_{0}} / \sqrt{m}$. We see explicitly that the formulation solely in terms of cavity vector fields is not sufficient for $m$-component spins, as we do not produce a partition function of a spin in a single field, alike to $\tilde{Z} \sim \operatorname{Tr} \exp \{\beta \tilde{\vec{h}} \tilde{\vec{s}}\}$ from equation A.1. Therefore, we confine ourselves to reproduce the spin expectation value of equation (A.5). That is what we call the self-consistent effective field approach.

To find $\left\langle\vec{s}_{0}\right\rangle_{c}$ resulting from equation (A.6), we use again a saddle point approach to solve the integral for the parameter $z$ from the integral representation of the $\delta$-constraint $\delta\left(m-{\overrightarrow{s_{0}}}^{2}\right)$, and additionally for the $m$ components of the spin $\overrightarrow{s_{0}}$. However, there is a mathematical subtlety involved. As the 
number of integration variables is $m+1$ and therefore of the same size as the large parameter $m$, the saddle point method can not be used. Nevertheless, it is possible to circumvent this problem. As the spins only occur as ${\overrightarrow{s_{0}}}^{2}$ and as $\overrightarrow{h_{i}}{\overrightarrow{s_{0}}}^{\prime}$ in the terms $J_{i}^{2}+2 \overrightarrow{J_{i}} \overrightarrow{h_{i}^{\prime}}{\overrightarrow{s_{0}}}^{\prime}+{\overrightarrow{h_{i}}}^{\prime 2}$, we can apply an orthogonal transformation $\vec{t}=O \vec{s}_{0}$, which projects the spin into the eigenbasis of the space of the cavity fields $\vec{h}_{i}$. This space is at most $k$-dimensional (since there are only $k$ fields $\vec{h}_{i}$ involved) and the product $O \vec{h}_{i} \vec{t}$ reduces from $m$ components to $k$. As we can carry out the remaining Gaussian integrals of the redundant $m-k$ components of $\vec{s}_{0}$ resp. $\vec{t}$, the saddle point method is applicable afterwards and we get

$$
\begin{aligned}
Z_{0} & =\int_{-i \infty}^{i \infty} \frac{d z}{2 \pi} \int d^{k} t \exp \left(m \sum _ { i = 1 } ^ { k } \left[\beta^{2}\left(\left(\vec{h}_{i}^{\prime}\right)^{2}+2 J_{i}\left(O \vec{h}_{i}^{\prime}\right) \vec{t}^{\prime}+J_{i}^{2}\right) / 4 z_{i}^{0}\right.\right. \\
& \left.\left.+z_{i}^{0}-\frac{1}{2} \ln z_{i}^{0}+z\left(1-\left(\vec{t}^{\prime}\right)^{2}\right)\right]-\frac{m-k}{2} \ln \frac{z}{\pi}\right) .
\end{aligned}
$$

The saddle point equations are

$$
\begin{aligned}
z & =\frac{1}{2\left(1-\left(\overrightarrow{t^{\prime}}\right)^{2}\right)} \\
2 z \vec{t} & =\sum_{i=1}^{k} \frac{2 \beta^{2} J_{i} O \vec{h}_{i}}{4 z_{i}^{0}} .
\end{aligned}
$$

In both equations $(A .8)$ and $A .9$ we can use the inverse orthogonal transformation. Multiplying equation (A.8) with $2 \vec{s}_{0}$, we have

$$
\frac{\left\langle\vec{s}_{0}\right\rangle_{c}}{1-\left\langle\vec{s}_{0}\right\rangle_{c}^{2}}=\sum_{i=1}^{k} \frac{2 \beta^{2} J_{i} O \vec{h}_{i}}{4 z_{i}^{0}} .
$$

As the definition of a saddle point is to produce the dominating weight of the partition function, the saddle point value of $\vec{t}$, resp. $\vec{s}_{0}$ we have just found is indeed the expectation value of $\vec{s}_{0},\left\langle\vec{s}_{0}\right\rangle_{c}$. Therefore, we have substituted it in equation (A.10). Now, we can connect this result to the expectation value from equation (A.5) of the spin $\vec{s}_{0}$ in a single field $\vec{h}_{0}$. Equation $(\vec{A} .5)$ can be rewritten as $\beta \overrightarrow{h_{0}}=\frac{\left\langle\vec{s}_{0}\right\rangle_{c}}{1-\left\langle\vec{s}_{0}\right\rangle_{c}^{2}}$ and in combination with equation A.100 we find

$$
\vec{h}_{0}=\sum_{i=1}^{k} \frac{2 \beta J_{i} \vec{h}_{i}}{4 z_{i}^{0}}
$$

where we substitute the spin expectation value $\left\langle\vec{s}_{0}\right\rangle_{c}$ from equation A.5 as well in $z_{i}^{0}=\frac{1}{4}\left(1+\sqrt{1+4 \beta^{2}\left(J_{i}^{2}+2 J_{i}\left\langle\overrightarrow{s_{0}^{\prime}}\right\rangle_{c}{\overrightarrow{h_{i}^{\prime}}}_{i}+{\overrightarrow{h_{i}^{\prime}}}^{\prime 2}\right)}\right)$, which makes it a 
complicated implicit equation for $\vec{h}_{0}$. However, it finally reveals the effective cavity field $\vec{h}_{0}$, which produces the same spin expectation value as the cavity partition function from equation (2.6). 


\section{Appendix B}

\section{Appendix to Section 5.3}

\section{B.1 Additional expression for $g_{n}^{k}(\vec{\sigma})$}

A second useful expression for $g_{n}^{k}(\vec{\sigma})$ from equation $(4.9)$ is derived by expanding $\exp \left(k \ln g_{n}(\vec{\sigma})\right)$ with $g_{n}(\vec{\sigma})$ from equation (4.9) and separating the overlaps into the zeroth order contribution $q_{\alpha \beta}^{\mu \nu(0)}$ in $\frac{1}{k}$ and into the first order contribution $q_{\alpha \beta}^{\mu \nu(1)}$ as (and analoguously for the four-spin overlaps).

$$
\begin{aligned}
g_{n}^{k}(\vec{\sigma}) & =\exp \left(k \ln \left(1+\frac{\lambda^{2}}{2 k} \sum_{\substack{\alpha, \beta \\
\mu, \nu}} q_{\alpha \beta}^{\mu \nu} \sigma_{\alpha}^{\mu} \sigma_{\beta}^{\nu}+\frac{\lambda^{4}}{24 k^{2}} \sum_{\substack{\alpha, \beta, \gamma, \delta \\
\mu, \nu, \eta, \rho}} q_{\alpha \beta \gamma \delta}^{\mu \nu \eta \rho} \sigma_{\alpha}^{\mu} \sigma_{\beta}^{\nu} \sigma_{\gamma}^{\eta} \sigma_{\delta}^{\rho}\right)\right) \\
& =\exp \left[k \left(\frac{\lambda^{2}}{2 k} \sum_{\substack{\alpha, \beta \\
\mu, \nu}} q_{\alpha \beta}^{\mu \nu} \sigma_{\alpha}^{\mu} \sigma_{\beta}^{\nu}+\frac{\lambda^{4}}{24 k^{2}} \sum_{\substack{\alpha, \beta, \gamma, \delta \\
\mu, \nu, \eta, \rho}} q_{\alpha \beta \gamma \delta}^{\mu \nu \eta \rho} \sigma_{\alpha}^{\mu} \sigma_{\beta}^{\nu} \sigma_{\gamma}^{\eta} \sigma_{\delta}^{\rho}\right.\right. \\
& \left.\left.-\frac{1}{2}\left(\frac{\lambda^{2}}{2 k} \sum_{\substack{\alpha, \beta \\
\mu, \nu}} q_{\alpha \beta}^{\mu \nu} \sigma_{\alpha}^{\mu} \sigma_{\beta}^{\nu}+\frac{\lambda^{4}}{24 k^{2}} \sum_{\substack{\alpha, \beta, \gamma, \delta \\
\mu, \nu, \eta, \rho}} q_{\alpha \beta \gamma \delta}^{\mu \nu \eta \rho} \sigma_{\alpha}^{\mu} \sigma_{\beta}^{\nu} \sigma_{\gamma}^{\eta} \sigma_{\delta}^{\rho}\right)^{2}+\mathcal{O}\left(\frac{1}{k^{3}}\right)\right)\right] \\
& =\exp \left[k \left(\frac{\lambda^{2}}{2 k} \sum_{\substack{\alpha, \beta \\
\mu, \nu}}\left(q_{\alpha \beta}^{\mu \nu(0)}+\frac{1}{k} q_{\alpha \beta}^{\mu \nu(1)}\right) \sigma_{\alpha}^{\mu} \sigma_{\beta}^{\nu}+\frac{\lambda^{4}}{24 k^{2}} \sum_{\substack{\alpha, \beta, \gamma, \delta \\
\mu, \nu, \eta, \rho}} q_{\alpha \nu \beta \gamma \delta}^{\mu \nu \eta(0)} \sigma_{\alpha}^{\mu} \sigma_{\beta}^{\nu} \sigma_{\gamma}^{\eta} \sigma_{\delta}^{\rho}\right.\right. \\
& \left.\left.-\frac{1}{2}\left(\frac{\lambda^{2}}{2 k} \sum_{\substack{\alpha, \beta \\
\mu, \nu}} q_{\alpha \beta}^{\mu \nu(0)} \sigma_{\alpha}^{\mu} \sigma_{\beta}^{\nu}\right)^{2}++\mathcal{O}\left(\frac{1}{k^{3}}\right)\right)\right]
\end{aligned}
$$

We separate the first term, $\exp \left(\frac{\lambda^{2}}{2} \sum_{\mu, \nu}^{\alpha, \beta} q_{\alpha \beta}^{\mu \nu(0)} \sigma_{\alpha}^{\mu} \sigma_{\beta}^{\nu}\right)$, from the rest, for 
which we expand the exponential and get

$$
\begin{aligned}
= & \exp \left(\frac{\lambda^{2}}{2} \sum_{\substack{\alpha, \beta \\
\mu, \nu}} q_{\alpha \beta}^{\mu \nu(0)} \sigma_{\alpha}^{\mu} \sigma_{\beta}^{\nu}\right)\left(1+\frac{\lambda^{2}}{2 k} \sum_{\substack{\alpha, \beta \\
\mu, \nu}} q_{\alpha \beta}^{\mu \nu(1)} \sigma_{\alpha}^{\mu} \sigma_{\beta}^{\nu}\right. \\
& \left.+\frac{\lambda^{4}}{24 k} \sum_{\substack{\alpha, \beta, \gamma, \delta \\
\mu, \nu, \eta, \rho}} q_{\alpha \beta \gamma \delta}^{\mu \nu \eta \rho(0)} \sigma_{\alpha}^{\mu} \sigma_{\beta}^{\nu} \sigma_{\gamma}^{\eta} \sigma_{\delta}^{\rho}-\frac{\lambda^{4}}{8 k}\left(\sum_{\substack{\alpha, \beta \\
\mu, \nu}} q_{\alpha \beta}^{\mu \nu(0)} \sigma_{\alpha}^{\mu} \sigma_{\beta}^{\nu}\right)^{2}+\mathcal{O}\left(\frac{1}{k^{2}}\right)\right) .
\end{aligned}
$$

It is feasible to expand this calculation to higher orders in $\frac{1}{k}$.

\section{B.2 Convenient form of the free energy}

We show how to derive a convenient expression for the free energy of the $m$ component spin glass on a Bethe lattice in terms of $g_{n}$. To this end we separate the known site- and bond-contributions tof the free energy on the Bethe lattice (see section 2.5) into effective field contributions $\vec{n}_{i j}$, which denote the effective field onto the spin $\vec{s}_{i}$ solely due to its neighbour $\vec{s}_{j}$. The usual free energy on a Bethe lattice is

$$
\begin{aligned}
F & =-k \sum_{i} F_{i}^{(1)}+\sum_{\langle i j\rangle} F_{i j}^{(2)} \\
& =-N k\left(-\frac{1}{\beta} \overline{\ln \operatorname{Tr} \rho_{i}^{(1)}}\right)+N \frac{k+1}{2}\left(-\frac{1}{\beta} \overline{\ln \operatorname{Tr} \rho_{i j}^{(2)}}\right)
\end{aligned}
$$

where

$$
\begin{aligned}
& \rho_{i}^{(1)}=\exp \left(\beta \sum_{j=1}^{k+1} \vec{\eta}_{i j} \vec{\sigma}_{i}\right) \\
& \rho_{i j}^{(2)}=\exp \left(\beta \sum_{l=1}^{k} \vec{\eta}_{i l} \vec{\sigma}_{i}+\beta \sum_{l=1}^{k} \vec{\eta}_{j l} \vec{\sigma}_{j}+\beta J_{i j} \vec{\sigma}_{i} \vec{\sigma}_{j}\right)
\end{aligned}
$$

Analoguously to chapter 2 we define a cavity field by summing over the single contributions, $\vec{\eta}_{i j}$, of all neighbouring spins but one as $\sum_{j=1}^{k} \vec{\eta}_{i j}=\vec{h}_{i}$ and the local field $\vec{H}_{i}$ as $\sum_{j=1}^{k+1} \vec{\eta}_{i j}=\vec{H}_{i}$ within this notation. Their distributions will enter the derivation of equation 4.11). We denote the distributions by $P^{(k+1)}(\vec{h})=Q(\vec{H})$ and $P^{(k)}(\vec{h})=Q(\vec{h})$ resp. (the index $k+1$ or $k$ resp. denotes the number of neighbours involved to define the corresponding field). 
The free energy per spin can be written as

$$
\begin{aligned}
\beta f & =k \int d^{m} h P^{(k+1)}(\vec{h}) \ln \operatorname{Tr}_{\sigma} e^{\beta \vec{H} \vec{\sigma}} \\
& -\frac{k+1}{2} E_{J} \int d^{m} h_{1} d^{m} h_{2} P^{(k)}\left(\vec{h}_{1}\right) P^{(k)}\left(\vec{h}_{2}\right) \\
& \times \ln \operatorname{Tr}_{\sigma_{1}, \sigma_{2}} e^{\beta J \vec{\sigma}_{1} \vec{\sigma}_{2}+\vec{h}_{1} \vec{\sigma}_{1}+\vec{h}_{2} \vec{\sigma}_{2}} .
\end{aligned}
$$

We see that the free energy of a spin glass on a Bethe lattice can be expressed by the distribution of cavity fields. In the same way we can calculate the free energy with the replica technique (by regarding $n$ copies of the same spin glass and sending $n$ to zero in the end). For instance the site-contribution is

$$
\overline{\ln \operatorname{Tr} \rho_{i}^{(1)}} \rightarrow \lim _{n \rightarrow 0} \frac{1}{n}\left(\overline{\ln \operatorname{Tr}_{\sigma_{i}^{\alpha}} \exp \left(\beta \sum_{j} \sum_{\alpha} \vec{\eta}_{i j}^{\alpha} \vec{\sigma}_{i}^{\alpha}\right)}\right) .
$$

For the bond-contribution there is a similar formulation for the replicated system. We define the cavity- and local fields for every single replica $\left(\vec{h}_{i}^{\alpha}\right)$ and extend the joint distribution to $k \times n$ fields (which we denote by $P_{n}^{(k)}\left(h^{\alpha}\right)$ ). The free energy is then found to be

$$
\begin{aligned}
n \beta f & =k \ln \operatorname{Tr}_{\sigma} \int \prod_{\alpha} d^{m} h^{\alpha} P_{n}^{(k+1)}\left(\vec{h}^{\alpha}\right) e^{\beta \sum_{\alpha} \vec{H}^{\alpha} \vec{\sigma}^{\alpha}} \\
& -\frac{k+1}{2} \ln \operatorname{Tr}_{\sigma_{1}^{\alpha}, \sigma_{2}^{\alpha}} E_{J} \\
& \times \int \prod_{\alpha} d^{m} h_{1}^{\alpha} d^{m} h_{2}^{\alpha} P_{n}^{(k)}\left(\vec{h}_{1}^{\alpha}\right) P_{n}^{(k)}\left(\vec{h}_{2}^{\alpha}\right) e^{\beta J \sum_{\alpha} \vec{\sigma}_{1}^{\alpha} \vec{\sigma}_{2}^{\alpha}+\vec{h}_{1}^{\alpha} \vec{\sigma}_{1}^{\alpha}+\vec{h}_{2}^{\alpha} \vec{\sigma}_{2}^{\alpha}}
\end{aligned}
$$

So far we have only reformulated the usual expression of the Bethe lattice free energy. Now, we define the weight function $g_{n}(\vec{\sigma})$, which we have already derived in the previous section, in a different, but consistent way (to equation (4.3)

$$
g_{n}^{k}(\vec{\sigma})=\int \prod_{\alpha} d^{m} h^{\alpha} P_{n}^{(k)}\left(\vec{h}^{\alpha}\right) e^{\beta \sum_{\alpha} \vec{h}^{\alpha} \vec{\sigma}^{\alpha}} .
$$

To see the consistency of both definitions of $g_{n}(\vec{\sigma})$, we have to understand that the conditional distribution $Q_{(z)}\left(\vec{\sigma}_{0} \mid \vec{s}^{(l)}\right)$ of finding $\vec{\sigma}_{0}$ given $\vec{s}^{(l)}$ (which is one of the $k+1$ neighbours of $\vec{\sigma}_{0}$ ) corresponds to the probability of finding $\vec{\sigma}_{0}$ calculated with the partition function of $\vec{\sigma}_{0}$ being subject to the effective field $\vec{\eta}_{0 l}$ (The effective field $\vec{\eta}_{0 l}$ is the field on to $\vec{\sigma}_{0}$ solely produced by the 
interaction with $\left.\vec{s}^{(l)}\right)$. With this correspondence we find the definitions of both order parameters $g_{n}$ and $g_{(z)}$ (from equation(4.3) ) to be equal

$$
\begin{aligned}
g_{(z)}^{k}(\vec{\sigma}) & =E_{J} \prod_{l}^{k} \operatorname{Tr}_{s}(l) \alpha \\
& =\int \prod_{\alpha} d_{(z)}\left({\overrightarrow{\sigma_{0}}}^{\alpha} \mid \vec{s}^{(l) \alpha}\right) \\
& \left.=\int \prod_{\alpha} P_{n} d^{m} \vec{\eta}^{\alpha}\right) \exp \left(\beta \sum_{\alpha} \sum_{i=1}^{k}{\overrightarrow{\eta_{0 i}}}^{\alpha}\right) \exp \left(\beta \sum_{\alpha}{\overrightarrow{\sigma_{0}}}^{\alpha}\right)
\end{aligned}
$$

The final step that leads to equation 4.11) is to insert the expression for $g_{n}^{k}(\vec{\sigma})$ of equation $\left.\mathrm{B} .8\right)$ (and the corresponding expression for $g_{n}^{k+1}(\vec{\sigma})$ ) into equation (B.7). However, we will additionally use an alternative formulation of the free energy, which we derive by making use of equation 4.6

$$
\begin{aligned}
n \beta f & =k \ln \operatorname{Tr}_{\sigma} g_{n}^{k+1}(\vec{\sigma})-\frac{k+1}{2} \ln \left(\operatorname{Tr}_{\tau} g_{n}^{k}(\vec{\tau}) \operatorname{Tr}_{\sigma} g_{n}^{k}(\vec{\sigma})\right. \\
& \left.\times \int d J \rho(J) \operatorname{Tr}_{\tau} \exp \left(\beta J \sum_{\alpha} \vec{\sigma}^{\alpha} \vec{\tau}^{\alpha}\right) g_{n}^{k}(\vec{\tau})\left(\operatorname{Tr}_{\tau} g_{n}^{k}(\vec{\tau})\right)^{-1}\right) \\
& =k \ln \operatorname{Tr} g_{n}^{k+1}-\frac{k}{2}\left(\ln \operatorname{Tr} g_{n}^{k}+\ln \operatorname{Tr} g_{n}^{k+1}\right)-\frac{1}{2}\left(\ln \operatorname{Tr} g_{n}^{k}+\ln \operatorname{Tr} g_{n}^{k+1}\right) \\
& =\frac{k}{2}\left(\ln \operatorname{Tr} g_{n}^{k+1}-\ln \operatorname{Tr} g_{n}^{k}\right)-\frac{1}{2}\left(\ln \operatorname{Tr} g_{n}^{k}+\ln \operatorname{Tr} g_{n}^{k+1}\right) .
\end{aligned}
$$

\section{B.3 The free energy in terms of the overlaps}

To split the free energy, we use equation (4.8) first of all for the first term in equation (B.10)

$$
\begin{aligned}
& \frac{k}{2} \ln \left[\operatorname{Tr}\left(1+\frac{\lambda^{2}}{2 k} \sum_{\substack{\alpha, \beta \\
\mu, \nu}} q_{\alpha \beta}^{\mu \nu} \sigma_{\alpha}^{\mu} \sigma_{\beta}^{\nu}+\frac{\lambda^{4}}{24 k^{2}} \sum_{\substack{\alpha, \beta, \gamma, \delta \\
\mu, \nu, \eta, \rho}} q_{\alpha \beta \gamma \delta}^{\mu \nu \eta \rho} \sigma_{\alpha}^{\mu} \sigma_{\beta}^{\nu} \sigma_{\gamma}^{\eta} \sigma_{\delta}^{\rho}\right) g_{n}^{k} / \operatorname{Tr} g_{n}^{k}\right] \\
= & \frac{k}{2} \ln \left(1+\frac{\lambda^{2}}{2 k} \sum_{\substack{\alpha, \beta \\
\mu, \nu}}\left(q_{\alpha \beta}^{\mu \nu}\right)^{2}+\frac{\lambda^{4}}{24 k^{2}} \sum_{\substack{\alpha, \beta, \gamma, \delta \\
\mu, \nu, \eta, \rho}}\left(q_{\alpha \beta \gamma \delta}^{\mu \nu \eta \rho}\right)^{2}\right) \\
= & \frac{\lambda^{2}}{4} \sum_{\substack{\alpha, \beta \\
\mu, \nu}}\left(\left(q_{\alpha \beta}^{\mu \nu(0)}\right)^{2}+\frac{2}{k} q_{\alpha \beta}^{\mu \nu(0)} q_{\alpha \beta}^{\mu \nu(1)}\right)+\frac{\lambda^{4}}{48 k} \sum_{\substack{\alpha, \beta, \gamma, \delta \\
\mu, \nu, \eta, \rho}}\left(q_{\alpha \beta \gamma \delta}^{\mu \nu \eta \rho(0)}\right)^{2}
\end{aligned}
$$

Further terms are of higher order either in $\frac{1}{k}$ or in $n$ (for the replica limit, we only need to know the leading order in $n$ ). For the second term in equation 
B.10 we get:

$$
\begin{aligned}
& -\frac{1}{2} \ln \left(\operatorname{Tr} g_{n}^{k}\right)-\frac{1}{2}\left(\ln \left(\operatorname{Tr} g_{n}^{k}\right)+\ln \left(\frac{\operatorname{Tr} g_{n}^{k+1}}{\operatorname{Tr} g_{n}^{k}}\right)\right) \\
= & -\ln \left(\operatorname{Tr} g_{n}^{k}\right)-\frac{\lambda^{4}}{4 k} \sum_{\substack{\alpha, \beta \\
\mu, \nu}}\left(q_{\alpha \beta}^{\mu \nu(0)}\right)^{2},
\end{aligned}
$$

where the third term in the first line corresponds to equation (B.11), but we only account for the zeroth order term in $\frac{1}{k}$ of it, as there is no prefactor $k$ as in the derivation of equation (B.11). The logarithm of the Trace of $g_{n}^{k}$ is evaluated using equation (4.10) and equation (4.8) (with $g_{n}^{k} \propto$ $\exp \left(\frac{\lambda^{2}}{2} \sum_{\substack{\alpha, \beta \\, \mu \nu}}^{\mu \nu(0)} q_{\alpha}^{\mu} \sigma_{\beta}^{\nu}\right)$ in zeroth order $)$

$$
\begin{aligned}
& \ln \left(\operatorname{Tr} g_{n}^{k}\right)=\ln \left[\operatorname { T r } \operatorname { e x p } ( \frac { \lambda ^ { 2 } } { 2 } \sum _ { \substack { \alpha , \beta \\
\mu , \nu } } q _ { \alpha \beta } ^ { \mu \nu ( 0 ) } \sigma _ { \alpha } ^ { \mu } \sigma _ { \beta } ^ { \nu } ) \left(1+\frac{\lambda^{2}}{2 k} \sum_{\substack{\alpha, \beta \\
\mu, \nu}} q_{\alpha \beta}^{\mu \nu(1)} \sigma_{\alpha}^{\mu} \sigma_{\beta}^{\nu}\right.\right. \\
& \left.\left.+\frac{\lambda^{4}}{24 k} \sum_{\substack{\alpha, \beta, \gamma, \delta \\
\mu, \nu, \eta, \rho}} q_{\alpha \beta \gamma \delta}^{\mu \nu \eta \rho(0)} \sigma_{\alpha}^{\mu} \sigma_{\beta}^{\nu} \sigma_{\gamma}^{\eta} \sigma_{\delta}^{\rho}-\frac{\lambda^{4}}{8 k}\left(\sum_{\substack{\alpha, \beta \\
\mu, \nu}} q_{\alpha \beta}^{\mu \nu(0)} \sigma_{\alpha}^{\mu} \sigma_{\beta}^{\nu}\right)^{2}\right)\right] \\
& =\ln \left[\operatorname{Tr} \exp \left(\frac{\lambda^{2}}{2} \sum_{\substack{\alpha, \beta \\
\mu, \nu}} q_{\alpha \beta}^{\mu \nu(0)} \sigma_{\alpha}^{\mu} \sigma_{\beta}^{\nu}\right)\right]+\ln \left[\operatorname{Tr} \exp \left(\frac{\lambda^{2}}{2} \sum_{\substack{\alpha, \beta \\
\mu, \nu}} q_{\alpha \beta}^{\mu \nu(0)} \sigma_{\alpha}^{\mu} \sigma_{\beta}^{\nu}\right)\right. \\
& \times\left(1+\frac{\lambda^{2}}{2 k} \sum_{\substack{\alpha, \beta \\
\mu, \nu}} q_{\alpha \beta}^{\mu \nu(1)} \sigma_{\alpha}^{\mu} \sigma_{\beta}^{\nu}+\frac{\lambda^{4}}{24 k} \sum_{\substack{\alpha, \beta, \gamma, \delta \\
\mu, \nu, \eta, \rho}} q_{\alpha \beta \gamma \delta}^{\mu \nu \eta \rho(0)} \sigma_{\alpha}^{\mu} \sigma_{\beta}^{\nu} \sigma_{\gamma}^{\eta} \sigma_{\delta}^{\rho}\right. \\
& \left.\left.-\frac{\lambda^{4}}{8 k}\left(\sum_{\substack{\alpha, \beta \\
\mu, \nu}} q_{\alpha \beta}^{\mu \nu(0)} \sigma_{\alpha}^{\mu} \sigma_{\beta}^{\nu}\right)^{2}\right) /\left(\operatorname{Tr} \exp \left(\frac{\lambda^{2}}{2} \sum_{\substack{\alpha, \beta \\
\mu, \nu}} q_{\alpha \beta}^{\mu \nu(0)} \sigma_{\alpha}^{\mu} \sigma_{\beta}^{\nu}\right)\right)\right] \\
& =\ln \left[\operatorname{Tr} \exp \left(\frac{\lambda^{2}}{2} \sum_{\substack{\alpha, \beta \\
\mu, \nu}} q_{\alpha \beta}^{\mu \nu(0)} \sigma_{\alpha}^{\mu} \sigma_{\beta}^{\nu}\right)\right]+\ln \left[1+\frac{\lambda^{2}}{2 k} \sum_{\substack{\alpha, \beta \\
\mu, \nu}}\left(q_{\alpha \beta}^{\mu \nu(0)} q_{\alpha \beta}^{\mu \nu(1)}\right)\right. \\
& \left.+\frac{\lambda^{4}}{24 k} \sum_{\substack{\alpha, \beta, \gamma, \delta \\
\mu, \nu, \eta, \rho}}\left(q_{\alpha \beta \gamma \delta}^{\mu \nu \eta \rho(0)}\right)^{2}-\frac{\lambda^{4}}{8 k} \sum_{\substack{\alpha, \beta, \gamma, \delta \\
\mu, \nu, \eta, \rho}} q_{\alpha \beta \gamma \delta}^{\mu \nu \eta \rho(0)} q_{\alpha \beta}^{\mu \nu(0)} q_{\gamma \delta}^{\eta \rho(0)}\right]
\end{aligned}
$$

We insert the resulting terms, separate them according to their order in $\frac{1}{k}$ and get 


$$
\begin{aligned}
m n \beta f_{0} & =\frac{\lambda^{2}}{4} \sum_{\substack{\alpha, \beta \\
\mu, \nu}}\left(q_{\alpha \beta}^{\mu \nu(0)}\right)^{2}-\ln \operatorname{Tr} \exp \left(\frac{\lambda^{2}}{2} \sum_{\substack{\alpha, \beta \\
\mu, \nu}} q_{\alpha \beta}^{\mu \nu(0)} \sigma_{\alpha}^{\mu} \sigma_{\beta}^{\nu}\right) \\
m n \beta f_{1} & =-\frac{\lambda^{2}}{4} \sum_{\substack{\alpha, \beta \\
\mu, \nu}}\left(q_{\alpha \beta}^{\mu \nu(0)}\right)^{2}-\frac{\lambda^{4}}{48} \sum_{\substack{\alpha, \beta, \gamma, \delta \\
\mu, \nu, \eta, \rho}}\left(q_{\alpha \beta \gamma \delta}^{\mu \nu \eta \rho(0)}\right)^{2} \\
& +\frac{\lambda^{4}}{8} \sum_{\substack{\alpha, \beta, \gamma, \delta \\
\mu, \nu, \eta, \rho}} q_{\alpha \beta}^{\mu \nu(0)} q_{\gamma \delta}^{\eta \rho(0)} q_{\alpha \beta \gamma \delta}^{\mu \nu \eta \rho(0)}\left(\delta_{\alpha \beta \gamma \delta}^{\mu \nu \eta \rho}+\ldots\right)
\end{aligned}
$$

This computation can be extended to higher orders. We have calculated the free energy in order $\frac{1}{k^{2}}$ (which we use in section 4.3.3)

$$
\begin{aligned}
& m n \beta f_{2}=\frac{\lambda^{2}}{4} \sum_{\substack{\alpha, \beta \\
\mu, \nu}}\left(q_{\alpha \beta}^{\mu \nu(1)}\right)^{2}-\frac{\lambda^{4}}{48} \sum_{\substack{\alpha, \beta, \gamma, \delta \\
\mu, \nu, \eta, \rho}}\left(q_{\alpha \beta \gamma \delta}^{\mu \nu \eta \rho(0)}\right)^{2} \\
& -\frac{\lambda^{6}}{1440} \sum_{\substack{\alpha, \beta, \gamma, \delta, \theta, \phi \\
\mu, \nu, \eta, \rho, \Theta, \Phi}}\left(q_{\alpha \beta \gamma \delta \theta \phi}^{\mu \nu \eta \rho \Theta \Phi(0)}\right)^{2}-\frac{\lambda^{2}}{2} \sum_{\substack{\alpha, \beta \\
\mu, \nu}}\left(q_{\alpha \beta}^{\mu \nu(0)} q_{\alpha \beta}^{\mu \nu(1)}\right) \\
& +\frac{\lambda^{4}}{4}\left[\sum_{\substack{\alpha, \beta, \gamma, \delta \\
\mu, \nu, \eta, \rho}} q_{\alpha \beta}^{\mu \nu(0)} q_{\gamma \delta}^{\eta \rho(1)} q_{\alpha \beta \gamma \delta}^{\mu \nu \eta \rho(0)}+\frac{\lambda^{2}}{12} \sum_{\substack{\alpha, \beta, \gamma, \delta, \theta, \phi \\
\mu, \nu, \eta, \rho, \Theta, \Phi}} q_{\alpha \beta}^{\mu \nu(0)} q_{\gamma \delta \theta \phi}^{\eta \rho \Theta \Phi(0)} q_{\alpha \beta \gamma \delta \theta \phi}^{\mu \nu \eta \rho \Theta(0)}\right] \\
& -\frac{\lambda^{6}}{24} \sum_{\substack{\alpha, \beta, \gamma, \delta, \theta, \phi \\
\mu, \nu, \eta, \rho, \Theta, \Phi}} q_{\alpha \beta}^{\mu \nu(0)} q_{\gamma \delta}^{\eta \rho(0)} q_{\theta \phi}^{\Theta \Phi(0)} q_{\alpha \beta \gamma \delta \theta \phi}^{\mu \nu \eta \rho \Theta(0)} \\
& -\frac{1}{2}\left[\frac{\lambda^{4}}{4} \sum_{\substack{\alpha, \beta, \gamma, \delta \\
\mu, \nu, \eta, \rho}} q_{\alpha \beta}^{\mu \nu(1)} q_{\gamma \delta}^{\eta \rho(1)} q_{\alpha \beta \gamma \delta}^{\mu \nu \eta \rho(0)}+\frac{\lambda^{6}}{24} \sum_{\substack{\alpha, \beta, \gamma, \delta, \theta, \phi \\
\mu, \nu, \eta, \rho, \Theta, \Phi}} q_{\alpha \beta}^{\mu \nu(1)} q_{\gamma \delta \theta \phi}^{\eta \rho \Theta \Phi(0)} q_{\alpha \beta \gamma \delta \theta \phi}^{\mu \nu \eta \rho \Theta \Phi(0)}\right. \\
& -\frac{\lambda^{6}}{8} \sum_{\substack{\alpha, \beta, \gamma, \delta, \theta, \phi \\
\mu, \nu, \eta, \rho, \Theta, \Phi}} q_{\alpha \beta}^{\mu \nu(1)} q_{\gamma \delta}^{\eta \rho(0)} q_{\theta \phi}^{\Theta \Phi(0)} q_{\alpha \beta \gamma \delta \theta \phi}^{\mu \nu \eta \rho \Theta \Phi(0)}+\frac{\lambda^{8}}{576} \sum_{\substack{\alpha, \beta, \gamma, \delta, \theta, \phi, i, j \\
\mu, \nu, \eta, \rho, \Theta, \Phi, s, t}} q_{\alpha \beta \gamma \delta}^{\mu \nu \eta \rho(0)} q_{\theta \phi i j}^{\Theta \Phi s t(0)} q_{\alpha \beta \gamma \delta \theta \phi i j}^{\mu \nu \eta \rho \Theta \Phi s t(0)} \\
& +\frac{\lambda^{8}}{96} \sum_{\substack{\alpha, \beta, \gamma, \delta, \theta, \phi, i, j \\
\mu, \nu, \eta, \rho, \Theta, \Phi, s, t}} q_{\alpha \beta \gamma \delta}^{\mu \nu \eta \rho(0)} q_{\theta \phi}^{\Theta \Phi(0)} q_{i j}^{s t(0)} q_{\alpha \beta \gamma \delta \theta \phi i j}^{\mu \nu \eta \rho \Theta \Phi s t(0)} \\
& \left.+\frac{\lambda^{8}}{64} \sum_{\substack{\alpha, \beta, \gamma, \delta, \theta, \phi, i, j \\
\mu, \nu, \eta, \rho, \Theta, \Phi, s, t}} q_{\alpha \beta}^{\mu \nu(0)} q_{\gamma \delta}^{\eta \rho(0)} q_{\theta \phi}^{\Theta \Phi(0)} q_{i j}^{s t(0)} q_{\alpha \beta \gamma \delta \theta \phi i j}^{\mu \nu \eta \rho \Theta \Phi s(0)}\right] .
\end{aligned}
$$




\section{B.3.1 Computation for $m, k \rightarrow \infty$ and corrections in $\frac{1}{m}$}

First of all, we evaluate the Trace of $T$. To this end, we introduce the single vector $\vec{\sigma}$, which is a list of the spin components $\mu=1, \cdots, m$ of every single spin $\vec{\sigma}_{\alpha}, \alpha=1, \cdots, n$. Afterwards, we write the $\delta$-constraint in evaluating the Trace in $T$ in its integral representation $\delta\left(\left(\vec{\sigma}_{\alpha}\right)^{2}-m\right)=\int \frac{d z_{\alpha}}{2 \pi} e^{z_{\alpha}\left(\left(\vec{\sigma}_{\alpha}\right)^{2}-m\right)}$, and introduce a parameter $\epsilon$ to ensure convergence of the following Gaussian integrals. Then, we can calculate $T$ as (with $M_{\alpha \beta}^{\mu \nu}=2\left(\epsilon-z_{\alpha}\right) \delta_{\alpha \beta}-\lambda^{2} q_{\alpha \beta}^{\mu \nu}$ )

$$
\begin{aligned}
T & =\int\left(\prod_{\alpha=1}^{n} \frac{d z_{\alpha}}{\sqrt{2 \pi}}\right) e^{m \sum_{\alpha}\left(\epsilon-z_{\alpha}\right)} \int d^{m n} \sigma \exp \left(-\frac{1}{2} \sigma^{T} M\left(z_{\alpha}\right) \sigma\right) \\
& =\int\left(\prod_{\alpha} \frac{d z_{\alpha}}{\sqrt{2 \pi}}\right) e^{m \sum_{\alpha}\left(\epsilon-z_{\alpha}\right)} \frac{\sqrt{2 \pi}^{m n}}{\sqrt{\operatorname{det} M\left(z_{\alpha}\right)}}
\end{aligned}
$$

So far the calculation is not restricted to any special case. The integrals are solved by sadddle point methods. To evaluate corrections for finite component numbers, we are going to expand around the saddle point of $T$ in a power series in $\frac{1}{m}$. An expansion of the matrix term yields (with $z_{\alpha}=z+\Delta z_{\alpha}$, $M\left(z_{\alpha}\right)=M_{0}(z)+\Delta M\left(\Delta z_{\alpha}\right)=M_{0}+\Delta M\left(z_{\alpha}\right)$ and omitting the explicit dependence of $M_{0}$ on $\left.z\right)$

$$
\begin{aligned}
\ln \operatorname{det} M\left(z_{\alpha}\right) & =\operatorname{Tr} \ln M\left(z_{\alpha}\right) \\
& =\operatorname{Tr} \ln \left(M_{0}+\Delta M\left(z_{\alpha}\right)\right)=\operatorname{Tr} \ln \left(M_{0}\left(\mathbb{1}+M_{0}^{-1} \Delta M\left(z_{\alpha}\right)\right)\right) \\
& =\operatorname{Tr} \ln \left(M_{0}\right)+\operatorname{Tr} \ln \left(\mathbb{1}+M_{0}^{-1} \Delta M\left(z_{\alpha}\right)\right) \\
& =\operatorname{Tr} \ln \left(M_{0}\right)+\operatorname{Tr} \sum_{k=1}^{\infty} \frac{(-1)^{k+1}}{k}\left(M_{0}^{-1} \Delta M\left(z_{\alpha}\right)\right)^{k}
\end{aligned}
$$

With it, we get

$$
\begin{aligned}
& \operatorname{Tr} \exp \left(\frac{\lambda^{2}}{2} \sum_{\substack{\alpha, \beta \\
\mu, \nu}} q_{\alpha \beta}^{\mu \nu(0)} \sigma_{\alpha}^{\mu} \sigma_{\beta}^{\nu}\right)=\int\left(\prod_{\alpha=1}^{n} \frac{d z_{\alpha}}{\sqrt{2 \pi}}\right) e^{m \sum_{\alpha}\left(\epsilon-\left(z+\Delta z_{\alpha}\right)\right)} \\
& \times \exp \left(-\frac{m}{2} \operatorname{Tr} \ln \left(M_{0}\right)-\frac{m}{2} \operatorname{Tr} M_{0}^{-1} \Delta M\left(z_{\alpha}\right)\right. \\
& \left.+\frac{m}{4} \operatorname{Tr}\left(M_{0}^{-1} \Delta M\left(z_{\alpha}\right)\right)^{2}+\frac{m}{2} \sum_{k=3}^{\infty} \frac{(-1)^{k+1}}{k} \operatorname{Tr}\left(M_{0}^{-1} \Delta M\left(z_{\alpha}\right)\right)^{k}\right) .
\end{aligned}
$$

The saddle point equation for the parameter $z$ is

$$
1-\operatorname{Tr} M_{0}^{-1}(z)=0
$$


which cancels the first order terms in $\Delta z_{\alpha}, m \sum \Delta z_{\alpha}$ and $-\frac{m}{2} \operatorname{Tr} M_{0}^{-1} \Delta M\left(z_{\alpha}\right)$ in equation (B.18). Then, equation (B.18) consists solely of Gaussian integrals in $\exp \left(\frac{m}{4} \operatorname{Tr}\left(M_{0}^{-1} \Delta M\left(z_{\alpha}\right)\right)^{2}\right)$. To see this, we note that the term $\Delta M\left(z_{\alpha}\right)$ is a matrix with the entries $2 \Delta z_{\alpha}$ on its diagonal. We expand the exponential $\exp \left[\frac{m}{2} \sum_{k=3}^{\infty} \frac{(-1)^{k+1}}{k} \operatorname{Tr}\left(M_{0}^{-1} \Delta M\left(z_{\alpha}\right)\right)^{k}\right]$ into its power series. This gives us the corrections in order $\frac{1}{\mathrm{~m}^{2}}$ and higher after solving the Gaussian integrals with the substitution $\Delta s_{\alpha}=\sqrt{m} \Delta M\left(z_{\alpha}\right)$ (which yields $\exp \left(\frac{m}{4} \operatorname{Tr}\left(M_{0}^{-1} \Delta M\left(z_{\alpha}\right)\right)^{2}\right) \rightarrow$ $\exp \left(\frac{1}{4} \operatorname{Tr}\left(M_{0}^{-1}\right)^{2} \Delta s_{\alpha}^{2}\right)$ such that it is clearly a Gaussian integral). The resulting contributions to $\ln T$ (up to fourth order) are

$$
\begin{aligned}
\ln T=m n\left(\epsilon-z-\frac{1}{2 n} \operatorname{Tr} \ln \left(M_{0}\right)\right. & -\frac{1}{2 n m} \ln \operatorname{Tr}\left(M_{0}^{-1}\right)^{2}+\frac{1}{n m^{2}} \ln T_{2} \\
& \left.+\frac{1}{n m^{3}} \ln T_{3}+\frac{1}{n m^{4}} \ln T_{4}+\ldots\right),
\end{aligned}
$$

with the corresponding expression for $T_{2}, T_{3}$ and $T_{4}$ given in the thesis.

\section{B.4 Calculating $q_{\alpha \beta}^{\mu \nu(0)}$ in spherical coordinates}

To prove that the overlap $q_{\alpha \beta}^{\mu \nu(0)}$ is different from zero only if $\mu=\nu$, we calculate $q_{\alpha^{\prime} \alpha^{\prime}}^{\mu \nu(0)}$ using spherical coordinates (for the calculation of $q_{\alpha \beta}^{(0)}$ see Ref. [41]). We align the $z$-axis along the spin $\vec{\sigma}_{\alpha^{\prime}}$. The single components of this spin are $\sigma_{\alpha^{\prime}}^{\mu}=\sqrt{m} \cos \Theta_{\alpha^{\prime}, m-2}$ and $\sigma_{\alpha^{\prime}}^{\nu}=\sqrt{m} \cos \Theta_{\alpha^{\prime}, m-3} \sin \Theta_{\alpha^{\prime}, m-2}$. The Jacobian of the transfomation into spherical coordinates is $\prod_{\alpha} \sin \Theta_{\alpha, 1}\left(\sin \Theta_{\alpha, 2}\right)^{2} \cdots\left(\sin \Theta_{\alpha, m-2}\right)^{m-2}$. The transformation makes the product $\vec{\sigma}_{\alpha^{\prime}} \vec{\sigma}_{\beta}$ to be equal to $\cos \Theta_{\beta, m-2}$, such that the exponent in the following calculation is independent of the angles 
$\Theta$ of replica $\alpha^{\prime}$. We then have

$$
\begin{aligned}
& q_{\alpha^{\prime} \alpha^{\prime}}^{\mu \nu}=\int d^{m n} \sigma \sigma_{\alpha^{\prime}}^{\mu} \sigma_{\alpha^{\prime}}^{\nu} e^{\frac{\lambda^{2}}{2} \sum q_{\alpha \beta} \vec{\sigma}_{\alpha} \vec{\sigma}_{\beta}}\left(\operatorname{Tr} \exp \left(\frac{\lambda^{2}}{2} \sum q_{\alpha \beta} \vec{\sigma}_{\alpha} \vec{\sigma}_{\beta}\right)\right)^{-1} \\
& =\int d^{n} r \prod_{\alpha}\left(\sqrt{m}^{m-1}\right) \delta\left(r^{2}-m\right) \int d^{n} \phi \int d^{n} \Theta_{1} \prod_{\alpha}\left(\sin \Theta_{\alpha, 1}\right) \cdots d^{n} \Theta_{m-2} \prod_{\alpha}\left(\left(\sin \Theta_{\alpha, m-2}\right)^{m-2}\right)
\end{aligned}
$$

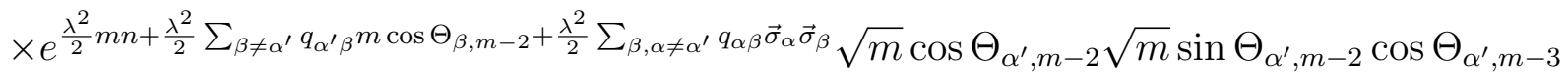

$$
\begin{aligned}
& \times\left(\int d^{n} r \prod_{\alpha}\left(\sqrt{m}^{m-1}\right) \delta\left(r^{2}-m\right) \int d^{n} \phi \int d^{n} \Theta_{1} \prod_{\alpha}\left(\sin \Theta_{\alpha, 1}\right) \cdots d^{n} \Theta_{m-2} \prod_{\alpha}\left(\left(\sin \Theta_{\alpha, m-2}\right)^{m-2}\right)\right.
\end{aligned}
$$

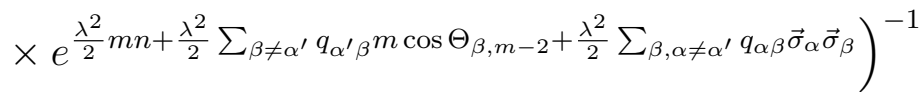

$$
\begin{aligned}
& =m \int d^{n-1} \Theta \prod_{\beta \neq \alpha^{\prime}}\left(\sin \Theta_{\beta, m-2}\right)^{m-2} e^{\frac{\lambda^{2}}{2} \sum_{\beta \neq \alpha^{\prime}} q_{\alpha^{\prime} \beta} m \cos \Theta_{\beta, m-2}} \\
& \times \int d \Theta_{\alpha^{\prime}, m-2}\left(\sin \Theta_{\alpha^{\prime}, m-2}\right)^{m-2} \cos \Theta_{\alpha^{\prime}, m-2} \sin \Theta_{\alpha^{\prime}, m-2} \int d \Theta_{\alpha^{\prime}, m-3}\left(\sin \Theta_{\alpha^{\prime}, m-3}\right)^{m-3} \cos \Theta_{\alpha^{\prime}, m-3} \\
& \times\left(\int d^{n-1} \Theta \prod_{\beta \neq \alpha^{\prime}}\left(\sin \Theta_{\beta, m-2}\right)^{m-2} e^{\frac{\lambda^{2}}{2} \sum_{\beta \neq \alpha^{\prime}} q_{\alpha^{\prime} \beta} m \cos \Theta_{\beta, m-2}}\right. \\
& \left.\times \int d \Theta_{\alpha^{\prime}, m-2}\left(\sin \Theta_{\alpha^{\prime}, m-2}\right)^{m-2} \int d \Theta_{\alpha^{\prime}, m-3}\left(\sin \Theta_{\alpha^{\prime}, m-3}\right)^{m-3}\right)^{-1} \\
& =0 \text {. }
\end{aligned}
$$

The last line follows after evaluating the integral for $\Theta_{\alpha^{\prime}, m-3}$. The same is true for four-spin overlaps which contain a single component different to the other ones. To show how the calculation of four-spin overlaps works we calculate the simplest instances, $q_{4}=\left\langle\left(\sigma_{\alpha}^{\mu}\right)^{4}\right\rangle$ and $q_{4}=\left\langle\left(\sigma_{\alpha}^{\mu}\right)^{2}\left(\sigma_{\alpha}^{\nu}\right)^{2}\right\rangle$. 


$$
\begin{aligned}
& q_{4}=\left\langle\left(\sigma_{\alpha^{\prime}}^{\mu}\right)^{4}\right\rangle \\
& =\int d^{n} r \prod_{\alpha}\left(\sqrt{m}^{m-1}\right) \delta\left(r^{2}-m\right) \int d^{n} \phi \int d^{n} \Theta_{1} \prod_{\alpha}\left(\sin \Theta_{\alpha, 1}\right) \cdots d^{n} \Theta_{m-2} \prod_{\alpha}\left(\left(\sin \Theta_{\alpha, m-2}\right)^{m-2}\right)
\end{aligned}
$$

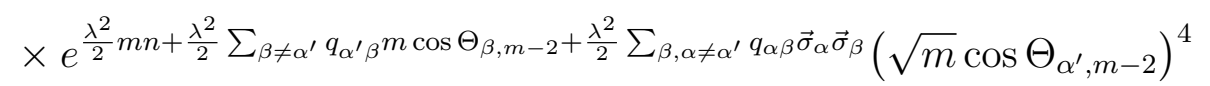

$$
\begin{aligned}
& \times\left(\int d^{n} r \prod_{\alpha}\left(\sqrt{m}^{m-1}\right) \delta\left(r^{2}-m\right) \int d^{n} \phi \int d^{n} \Theta_{1} \prod_{\alpha}\left(\sin \Theta_{\alpha, 1}\right) \cdots d^{n} \Theta_{m-2} \prod_{\alpha}\left(\left(\sin \Theta_{\alpha, m-2}\right)^{m-2}\right)\right. \\
& \times e^{\left.\frac{\lambda^{2}}{2} m n+\frac{\lambda^{2}}{2} \sum_{\beta \neq \alpha^{\prime}} q_{\alpha^{\prime} \beta} m \cos \Theta_{\beta, m-2}+\frac{\lambda^{2}}{2} \sum_{\beta, \alpha \neq \alpha^{\prime}} q_{\alpha \beta} \vec{\sigma}_{\alpha} \vec{\sigma}_{\beta}\right)^{-1}} \\
& =m^{2} \int d^{n-1} \Theta \prod_{\beta \neq \alpha^{\prime}}\left(\sin \Theta_{\beta, m-2}\right)^{m-2} e^{\frac{\lambda^{2}}{2} \sum_{\beta \neq \alpha^{\prime}} q_{\alpha^{\prime} \beta} m \cos \Theta_{\beta, m-2}} \\
& \times \int d \Theta_{\alpha^{\prime}, m-2}\left(\sin \Theta_{\alpha^{\prime}, m-2}\right)^{m-2}\left(\cos \Theta_{\alpha^{\prime}, m-2}\right)^{4} \\
& \times\left(\int d^{n-1} \Theta \prod_{\beta \neq \alpha^{\prime}}\left(\sin \Theta_{\beta, m-2}\right)^{m-2} e^{\frac{\lambda^{2}}{2} \sum_{\beta \neq \alpha^{\prime}} q_{\alpha^{\prime} \beta} m \cos \Theta_{\beta, m-2}}\right. \\
& \left.\times \int d \Theta_{\alpha^{\prime}, m-2}\left(\sin \Theta_{\alpha^{\prime}, m-2}\right)^{m-2}\right)^{-1} \\
& =\frac{3 m^{2}}{m+2} \frac{\int d \Theta_{\alpha^{\prime}, m-2}\left(\sin \Theta_{\alpha^{\prime}, m-2}\right)^{m-2}\left(\cos \Theta_{\alpha^{\prime}, m-2}\right)^{2}}{\int d \Theta_{\alpha^{\prime}, m-2}\left(\sin \Theta_{\alpha^{\prime}, m-2}\right)^{m-2}}=\frac{3 m^{2}}{m+2} .
\end{aligned}
$$




$$
\begin{gathered}
p_{4}=\left\langle\left(\sigma_{\alpha^{\prime}}^{\mu}\right)^{2}\left(\sigma_{\alpha^{\prime}}^{\nu}\right)^{2}\right\rangle \\
=\int d^{n} r \prod_{\alpha}\left(\sqrt{m}^{m-1}\right) \delta\left(r^{2}-m\right) \int d^{n} \phi \int d^{n} \Theta_{1} \prod_{\alpha}\left(\sin \Theta_{\alpha, 1}\right) \cdots d^{n} \Theta_{m-2} \prod_{\alpha}\left(\left(\sin \Theta_{\alpha, m-2}\right)^{m-2}\right) \\
\times\left(\sqrt{m} \cos \Theta_{\alpha^{\prime}, m-2}\right)^{2}\left(\sqrt{m} \cos \Theta_{\alpha^{\prime}, m-3} \sin \Theta_{\alpha^{\prime}, m-2}\right)^{2} \\
\quad \times e^{\frac{\lambda^{2}}{2} m n+\frac{\lambda^{2}}{2} \sum_{\beta \neq \alpha^{\prime}} q_{\alpha^{\prime} \beta} m \cos \Theta_{\beta, m-2}+\frac{\lambda^{2}}{2} \sum_{\beta, \alpha \neq \alpha^{\prime}} q_{\alpha \beta} \vec{\sigma}_{\alpha} \vec{\sigma}_{\beta}} \\
\times\left(\int d^{n} r \prod_{\alpha}\left(\sqrt{m}^{m-1}\right) \delta\left(r^{2}-m\right) \int d^{n} \phi \int d^{n} \Theta_{1} \prod_{\alpha}\left(\sin \Theta_{\alpha, 1}\right) \cdots d^{n} \Theta_{m-2} \prod_{\alpha}\left(\left(\sin \Theta_{\alpha, m-2}\right)^{m-2}\right)\right. \\
\quad \times e^{\left.\frac{\lambda^{2}}{2} m n+\frac{\lambda^{2}}{2} \sum_{\beta \neq \alpha^{\prime}} q_{\alpha^{\prime} \beta} m \cos \Theta_{\beta, m-2}+\frac{\lambda^{2}}{2} \sum_{\beta, \alpha \neq \alpha^{\prime}} q_{\alpha \beta} \vec{\sigma}_{\alpha} \vec{\sigma}_{\beta}\right)^{-1}} \\
=m^{2} \int d \Theta_{\alpha^{\prime}, m-2}\left(\sin \Theta_{\alpha^{\prime}, m-2}\right)^{m-2}\left(\cos \Theta_{\alpha^{\prime}, m-2} \sin \Theta_{\alpha^{\prime}, m-2}\right)^{2} \\
\times \int d \Theta_{\alpha^{\prime}, m-3}\left(\sin \Theta_{\alpha^{\prime}, m-3}\right)^{m-3}\left(\cos \Theta_{\alpha^{\prime}, m-3}\right)^{2} \\
\times\left(\int d \Theta_{\alpha^{\prime}, m-2}\left(\sin \Theta_{\alpha^{\prime}, m-2}\right)^{m-2} \int d \Theta_{\alpha^{\prime}, m-3}\left(\sin \Theta_{\alpha^{\prime}, m-3}\right)^{m-3}\right)^{-1} \\
=\frac{m^{2}}{m+2} \frac{\int d \Theta_{\alpha^{\prime}, m-2}\left(\sin \Theta_{\alpha^{\prime}, m-2}\right)^{m-2}\left(\cos \Theta_{\alpha^{\prime}, m-2}\right)^{2} d \Theta_{\alpha^{\prime}, m-3}\left(\sin \Theta_{\alpha^{\prime}, m-3}\right)^{m-3}}{\int d \Theta_{\alpha^{\prime}, m-2}\left(\sin \Theta_{\alpha^{\prime}, m-2}\right)^{m-2} \int d \Theta_{\alpha^{\prime}, m-3}\left(\sin \Theta_{\alpha^{\prime}, m-3}\right)^{m-3}} \frac{m^{2}}{m+2} .
\end{gathered}
$$

\section{B.5 Wick's theorem}

In this section we apply Wick's theorem to the case of the $m$-component spin glass in the limit $m \rightarrow \infty$. The theorem allows for the calculation of expectation values of higher order correlation functions of identically distributed Gaussian variables $\left(x_{a}, x_{b}, x_{c}, x_{d}\right)$ in terms of their two-point correlation functions, as $\left\langle x_{a} x_{b} x_{c} x_{d}\right\rangle=\left\langle x_{a} x_{b}\right\rangle\left\langle x_{c} x_{d}\right\rangle+\left\langle x_{a} x_{c}\right\rangle\left\langle x_{b} x_{d}\right\rangle+\left\langle x_{a} x_{d}\right\rangle\left\langle x_{b} x_{c}\right\rangle$, by taking all possible combinations into account.

In case of the $m$-component spin glass we want to use the theorem for the computation of four-spin overlaps. However they are not defined as Gaussian random variables. Therefore we can not directly use it for the 


\section{B.5. WICK'S THEOREM}

four-spin overlaps as for instance for $q_{4}=\left\langle\left(\sigma_{\alpha^{\prime}}^{\mu^{\prime}}\right)^{4}\right\rangle$

$$
\begin{aligned}
q_{4} & =\int d^{m n} \sigma \prod_{\alpha} \delta\left(\left(\vec{\sigma}_{\alpha}\right)^{2}-m\right)\left(\sigma_{\alpha^{\prime}}^{\mu^{\prime}}\right)^{4} e^{\frac{\lambda^{2}}{2} \sum q_{\alpha \beta} \vec{\sigma}_{\alpha} \vec{\sigma}_{\beta}} /\left(\operatorname{Tr} e^{\frac{\lambda^{2}}{2} \sum q_{\alpha \beta} \vec{\sigma}_{\alpha} \vec{\sigma}_{\beta}}\right) \\
& =\int \frac{d^{n} z}{(2 \pi)^{n}} \int d^{m n} \sigma e^{-\sum_{\alpha} z_{\alpha}\left(\left(\vec{\sigma}_{\alpha}\right)^{2}-m\right)}\left(\sigma_{\alpha^{\prime}}^{\mu^{\prime}}\right)^{4} e^{\frac{\lambda^{2}}{2} \sum q_{\alpha \beta} \vec{\sigma}_{\alpha} \vec{\sigma}_{\beta}} /\left(\operatorname{Tr} e^{\frac{\lambda^{2}}{2} \sum q_{\alpha \beta} \vec{\sigma}_{\alpha} \vec{\sigma}_{\beta}}\right) .
\end{aligned}
$$

At this point we can rewrite this expression in order to evaluate the fourspin overlap with a Gaussian weight, as $\left\langle\left(\sigma_{\alpha^{\prime}}^{\mu^{\prime}}\right)^{4}\right\rangle_{G, x}=\frac{\int d^{m n} \sigma\left(\sigma_{\alpha^{\prime}}^{\mu^{\prime}}\right)^{4} e^{-\sum z_{\alpha}\left(\vec{\sigma}_{\alpha}\right)^{2}+\frac{\lambda^{2}}{2} \sum q_{\alpha \beta} \vec{\sigma}_{\alpha} \vec{\sigma}_{\beta}}}{\int d^{m n} \sigma e^{-\sum z \alpha\left(\vec{\sigma}_{\alpha}\right)^{2}+\frac{\lambda^{2}}{2} \sum q_{\alpha \beta} \vec{\sigma}_{\alpha} \vec{\sigma}_{\beta}}}$ (the indices refer to a Gaussian expectation value depending on the saddle point value of $x_{\alpha}=2 z_{\alpha}-\lambda^{2} q_{2}+\lambda^{2} q_{11}$, omitting to explicitly introduce a factor $\epsilon$ for convergence of the Gaussian integrals)

$$
q_{4}=\int \frac{d^{n} z_{\alpha}}{(2 \pi)^{n}} e^{m \sum z_{\alpha}}\left\langle\left(\sigma_{\alpha^{\prime}}^{\mu^{\prime}}\right)^{4}\right\rangle_{G, x} \frac{e^{-\sum z_{\alpha}\left(\vec{\sigma}_{\alpha}\right)^{2}+\frac{\lambda^{2}}{2} \sum q_{\alpha \beta} \vec{\sigma}_{\alpha} \vec{\sigma}_{\beta}}}{\operatorname{Tr} e^{\frac{\lambda^{2}}{2} \sum q_{\alpha \beta} \vec{\sigma}_{\alpha} \vec{\sigma}_{\beta}}} .
$$

We apply Wick's theorem for $\left\langle\left(\sigma_{\alpha^{\prime}}^{\mu^{\prime}}\right)^{4}\right\rangle_{G, x}=3\left\langle\left(\sigma_{\alpha^{\prime}}^{\mu^{\prime}}\right)^{2}\right\rangle_{G, x}^{2}$ (note that the combinatorics require a closer look for the other four-spin overlaps, for instance for $\left.q_{211}\right)$. The expectation values of each two-spin overlap is known, it is $\left\langle\left(\sigma_{\alpha^{\prime}}^{\mu^{\prime}}\right)^{2}\right\rangle_{G, x}=q_{2}$ and $\left\langle\sigma_{\alpha^{\prime}}^{\mu^{\prime}} \sigma_{\beta^{\prime}}^{\mu^{\prime}}\right\rangle_{G, x}=q_{11}$ (which are finite). However, these overlaps do not contribute to the saddle point equation for $z_{\alpha}$, resp. $x_{\alpha}$ (as they are not of the order $e^{m n \cdots}$ ). Therefore the saddle point equation is the same for both the nominator and the denominator in equation B.25). It is $\frac{d}{d x_{\alpha}}\left(x_{\alpha}+\lambda^{2} q_{2}-\lambda^{2} q_{11}-\frac{1}{2} \ln \operatorname{det} M\left(x_{\alpha}\right)\right)=0$ with the solution $x_{0}=\frac{1}{2}+\sqrt{\frac{1}{4}+\lambda^{2} q_{11}}$, see the calculation of $q_{\alpha \beta}$ within the thesis. Finally these contributions cancel and we get

$$
q_{4}=3 q_{2}^{2}
$$

The results for other overlaps are reported in equation 4.20). They hold as long as using the saddle point method for $m \rightarrow \infty$ in this context is correct (i.e. that the terms not regarded to the saddle point never become too large), and our result is the same as if we had used Wick's theorem directly. This procedure is supported by the findings of the previous section that $q_{4}=\frac{3 m}{m+2}$ and $p_{4}=\frac{m}{m+2}$, which simplifies to $3=3 q_{2}^{2}$ (from $\left\langle\left(\sigma_{\alpha}^{\mu}\right)^{4}\right\rangle=3\left\langle\left(\sigma_{\alpha}^{\mu}\right)^{2}\right\rangle^{2}$ ) resp. $1=q 2^{2}\left(\left\langle\left(\sigma_{\alpha}^{\mu}\right)^{2}\left(\sigma_{\alpha}^{\nu}\right)^{2}\right\rangle=\left\langle\left(\sigma_{\alpha}^{\mu}\right)^{2}\right\rangle^{2}\right.$, as the overlap $q_{\alpha \beta}^{\mu \nu}$ is only different from zero for $\mu=\nu)$ in the limit $m \rightarrow \infty$. 


\section{B.6 Diagonalisation and inverse of $M_{0}$}

\section{B.6.1 Replica Symmetry}

We illustrate the matrices $M_{0}$ and $M_{0}^{-1}$ in a short hand notation with the simplification $n=2$ and $m=2$. It is

$$
M_{0}=\left(\begin{array}{cccc}
x-\lambda^{2} q_{11} & 0 & -\lambda^{2} q_{11} & 0 \\
0 & x-\lambda^{2} q_{11} & 0 & -\lambda^{2} q_{11} \\
-\lambda^{2} q_{11} & 0 & x-\lambda^{2} q_{11} & 0 \\
0 & -\lambda^{2} q_{11} & 0 & x-\lambda^{2} q_{11}
\end{array}\right)
$$

and

$$
M_{0}^{-1}=\left(\begin{array}{cccc}
\frac{1}{x}+\frac{\lambda^{2} q_{11}}{x^{2}} & 0 & \frac{\lambda^{2} q_{11}}{x^{2}} & 0 \\
0 & \frac{1}{x}+\frac{\lambda^{2} q_{11}}{x^{2}} & 0 & \frac{\lambda^{2} q_{11}}{x^{2}} \\
\frac{\lambda^{2} q_{11}}{x^{2}} & 0 & \frac{1}{x}+\frac{\lambda^{2} q_{11}}{x^{2}} & 0 \\
0 & \frac{\lambda^{2} q_{11}}{x^{2}} & 0 & \frac{1}{x}+\frac{\lambda^{2} q_{11}}{x^{2}}
\end{array}\right)
$$

To set $n$ to two is an oversimplification. To confirm that $\left(M_{0}\right)\left(M_{0}\right)^{-1}=\mathbb{1}$, we have to be careful to take the replica limit $n \rightarrow 0$. For instance we find $\left(M_{0}\right)\left(M_{0}\right)_{11}^{-1}=\left(x-\lambda^{2} q_{11}\right)\left(\frac{1}{x}+\frac{\lambda^{2} q_{11}}{x^{2}}\right)-(n-1) \lambda^{2} q_{11} \frac{\lambda^{2} q_{11}}{x^{2}}=1$ and $\left(M_{0}\right)\left(M_{0}\right)_{13}^{-1}=$ $\left(x-\lambda^{2} q_{11}\right) \frac{\lambda^{2} q_{11}}{x^{2}}-\lambda^{2} q_{11}\left(\frac{1}{x}+\frac{\lambda^{2} q_{11}}{x^{2}}\right)-(n-2) \lambda^{2} q_{11} \frac{\lambda^{2} q_{11}}{x^{2}}=0$

The diagonalisation is done by finding the eigenvalues $x$ and $x-n \lambda^{2} q_{11}^{(0)}$ with the orthonormal eigenvectors (each of the vectors has degeneracy $m$ and the vertical dots represent $m-3$ entries which are zero such that there is a 
single non-zero entry for each replica)

$$
\left(\begin{array}{c}
\frac{1}{\sqrt{n}} \\
0 \\
\vdots \\
0 \\
\frac{1}{\sqrt{n}} \\
0 \\
\vdots \\
0 \\
\frac{1}{\sqrt{n}} \\
\vdots
\end{array}\right), \quad \text { and }\left(\begin{array}{c}
\frac{1}{\sqrt{2}} \\
0 \\
\vdots \\
0 \\
-\frac{1}{\sqrt{2}} \\
0 \\
\vdots \\
0 \\
0 \\
\vdots
\end{array}\right) \text { and }\left(\begin{array}{c}
\frac{1}{\sqrt{2^{2}+2}} \\
0 \\
\vdots \\
0 \\
\frac{1}{\sqrt{2^{2}+2}} \\
0 \\
\vdots \\
0 \\
-\frac{2}{\sqrt{2^{2}+2}} \\
0 \\
\vdots \\
0 \\
0 \\
\vdots
\end{array}\right) \quad \ldots \quad\left(\begin{array}{c}
\frac{1}{\sqrt{(n-1)^{2}+n-1}} \\
0 \\
\vdots \\
0 \\
\frac{1}{\sqrt{(n-1)^{2}+n-1}} \\
\vdots \\
\vdots \\
\frac{n-1}{\sqrt{(n-1)^{2}+n-1}} \\
0 \\
\vdots \\
0
\end{array}\right) .
$$

The first $m$ eigenvectors belong to the eigenvalue $\propto x-n \lambda^{2} q_{11}^{(0)}$ and the other $m(n-1)$ vectors to the eigenvalue $\propto x$. The inverse of $M_{0}$ is calculated by the orthogonal transformation $M_{0}^{-1}=U D^{-1} U^{T}$ with $D$ being the diagonal matrix of $M_{0}$ and $U$ being the orthonormal matrix consisting of columns which correspond to the normalized eigenvectors of $M_{0}$. As can be seen from equation (4.14) and 4.22 we have to calculate various powers $t$ of $\operatorname{Tr}\left(M_{0}^{-1}\right)^{t}$ and their derivations with respect to $q_{11}^{(0)}$. The explicit expressions of this terms are not very enlightening and we omit to present them explicitly.

\section{B.6.2 1-RSB}

In case of 1-RSB the diagonalisation of $M_{0}$ is done similar to above (with $\left.x \rightarrow y=2(\epsilon-z)-\lambda^{2} q_{2,2}-\lambda^{2} q_{11,2}\right)$. An example for $M_{0}$ with $n=4, m=1$ and $r=2$ is

$$
M_{0}=\left(\begin{array}{cccc}
y-\lambda^{2} q_{11,2} & -\lambda^{2} q_{11,2} & -\lambda^{2} q_{11,11} & -\lambda^{2} q_{11,11} \\
-\lambda^{2} q_{11,2} & y-\lambda^{2} q_{11,2} & -\lambda^{2} q_{11,11} & -\lambda^{2} q_{11,11} \\
-\lambda^{2} q_{11,11} & y-\lambda^{2} q_{11,11} & y-\lambda^{2} q_{11,2} & \lambda^{2} q_{11,2} \\
-\lambda^{2} q_{11,11} & y-\lambda^{2} q_{11,11} & -\lambda^{2} q_{11,2} & y-\lambda^{2} q_{11,2}
\end{array}\right) .
$$

Additionally to the eigenvalues $\propto y$ (with the first $m$ eigenvectors in eq. (B.31) and $\propto y-r \lambda^{2}\left(q_{11,2}-q_{11,11}\right)-n \lambda^{2} q_{11,11}$ (with the other (only!) $m(r-1) \frac{n}{r}$ 
eigenvectors) from the replica symmetric matrix in equation (B.31), we find the eigenvectors

$$
\left(\begin{array}{c}
\frac{1}{\sqrt{2}} \\
0 \\
\vdots \\
0 \\
-\frac{1}{\sqrt{2}} \\
0 \\
\vdots \\
0 \\
0 \\
\vdots
\end{array}\right) \quad \text { and }\left(\begin{array}{c}
\frac{1}{\sqrt{2^{2}+2}} \\
0 \\
\vdots \\
0 \\
\frac{1}{\sqrt{2^{2}+2}} \\
0 \\
\vdots \\
0 \\
-\frac{2}{\sqrt{2^{2}+2}} \\
0 \\
\vdots \\
0 \\
0 \\
\vdots
\end{array}\right) \quad \cdots\left(\begin{array}{c}
\frac{1}{\sqrt{(n / r-1)^{2}+n / r-1}} \\
0 \\
\vdots \\
0 \\
\frac{1}{\sqrt{(n / r-1)^{2}+n / r-1}} \\
\vdots \\
\vdots \\
-\frac{n / r-1}{\sqrt{(n / r-1)^{2}+n / r-1}} \\
0 \\
\vdots \\
0
\end{array}\right)
$$

to the eigenvalue $\propto y-r \lambda^{2}\left(q_{11,2}-q_{11,11}\right)$ with degeneracy $m\left(\frac{n}{r}-1\right)$ (The vertical dots represent $m r-3$ entries which are zero such that there is a single non-zero entry for each block of replicas). The inverse matrix is (again depicted for $n=4, m=1$ and $r=2$ )

$M_{0}^{-1}=\left(\begin{array}{cccc}\frac{r-1}{y r}+\frac{n / r-1}{x_{2} n}+\frac{1}{x_{3} n} & -\frac{1}{y r}+\frac{n / r-1}{x_{2} n}+\frac{1}{x_{3} n} & -\frac{1}{x_{2} n}+\frac{1}{x_{3} n} & -\frac{1}{x_{2} n}+\frac{1}{x_{3} n} \\ -\frac{1}{y r}+\frac{n / r-1}{x_{2} n}+\frac{1}{x_{3} n} & \frac{r-1}{y r}+\frac{n / r-1}{x_{2} n}+\frac{1}{x_{3} n} & -\frac{1}{x_{2} n}+\frac{1}{x_{3} n} & -\frac{1}{x_{2} n}+\frac{1}{x_{3} n} \\ -\frac{1}{x_{2} n}+\frac{1}{x_{3} n} & -\frac{1}{x_{2} n}+\frac{1}{x_{3} n} & \frac{r-1}{y r}+\frac{n / r-1}{x_{2} n}+\frac{1}{x_{3} n} & -\frac{1}{y r}+\frac{n / r-1}{x_{2} n}+\frac{1}{x_{3} n} \\ -\frac{1}{x_{2} n}+\frac{1}{x_{3} n} & -\frac{1}{x_{2} n}+\frac{1}{x_{3} n} & -\frac{1}{y r}+\frac{n / r-1}{x_{2} n}+\frac{1}{x_{3} n} & \frac{r-1}{y r}+\frac{n / r-1}{x_{2} n}+\frac{1}{x_{3} n}\end{array}\right)$.

We again omit the explicit expressions of the various powers of $M_{0}^{-1}$ and its derivations with respect to $q_{11,2}^{(0)}, q_{11,11}^{(0)}$ and $y_{0}$ ( $y_{0}$ is an additional variable in the 1-RSB case), which we have to use in the corresponding equations to (4.14) and (4.22) that determine $q_{11,2}^{(0)}, q_{11,11}^{(0)}$ and $y_{0}$ up to fourth order in $\frac{1}{m}$ in the $1-\mathrm{RSB}$ case. 


\section{B.6.3 One step broken replica symmetry solution}

We exemplify the computations in case of 1-RSB by the determining equation for the overlap $q_{11,2}^{(00)}$ in zeroth order

$$
\begin{aligned}
\frac{\lambda^{2}}{2} m n(r-1) q_{11,2}^{(00)} & =\left.\frac{d}{d q_{11,2}^{(0)}} \ln T_{0}^{1 R S B}\right|_{q_{11, x}^{(0)}=q_{11, x}^{(00)}} \\
& =\frac{m n}{2}\left(-\lambda^{2}+\frac{\lambda^{2}}{y_{0}-r \lambda^{2} \Delta q}+\frac{r \lambda^{4} q_{11,11}^{(0)}}{\left(y_{0}-r \lambda^{2} \Delta q\right)^{2}}\right. \\
& \left.+\mathcal{O}_{0}\left(\frac{d y_{0}}{d q_{11,2}^{(0)}}\right)\left(1-\frac{1}{y_{0}}+\frac{1}{r y_{0}}-\frac{1}{r\left(y_{0}-r \lambda^{2} \Delta q\right)}-\frac{\lambda^{2} q_{11,11}^{(0)}}{\left(y_{0}-r \lambda^{2} \Delta q\right)^{2}}\right)\right)\left.\right|_{q_{11, x}^{(0)}=q_{11, x}^{(00)}} \\
\Rightarrow & (r-1) q_{11,2}^{(00)}=-1+\frac{1}{y_{00}-r \lambda^{2} \Delta q_{0}}+\frac{r \lambda^{2} q_{11,11}^{(00)}}{\left(y_{00}-r \lambda^{2} \Delta q_{0}\right)^{2}} .
\end{aligned}
$$

The penultimate line is the determining equation for all higher orders in $\frac{1}{m}$ besides that we have to add higher order terms of $\ln T$ as in equation (4.22) of the replica symmetric calculation. For $q_{11,11}^{(0)}$ this equation is equation 4.29) and for $y_{0}$ it is equation 4.26) $\left(\operatorname{saddle}_{y}\left(q_{11,2}^{(0)}, q_{11,11}^{(0)}\right)\right)$.

\section{B.7 Maximizing the free energies with respect to $r$}

The free energy in terms of the overlaps $q_{\alpha \beta}^{\mu \nu}$ is written down in equation (5.24). Since we have used the equation of "motion" (4.8) to write down $f_{1}$ in this way, this part of the free energy is no longer stationary with respect to the $q$ 's in the 1-RSB case. Nevertheless it still gives the correct value for the free energy. Therefore it is only necessary to minimize $f_{0}$ with respect to $r$ and $\alpha_{2}$. To do so, we use the following equation

$$
\begin{gathered}
m n \beta f_{0}=m n \frac{\lambda^{2}}{4}\left(\left(q_{2,2}\right)^{2}+(r-1)\left(q_{11,2}\right)^{2}+(n-r)\left(q_{11,11}\right)^{2}\right)-\frac{n}{2} \ln \left(\frac{2}{m}\right) \\
-\frac{m n}{2}\left(y_{0}+\lambda^{2} q_{2,2}-\lambda^{2} q_{11,2}\right) \\
+\frac{m}{2} \operatorname{Tr} \ln M_{0}+\frac{n}{2} \operatorname{Tr}\left(M_{0}^{-1}\right)^{2}+\frac{1}{m}\left(\frac{3 \operatorname{Tr}\left(M_{0}^{-1}\right)^{4}}{\left(\operatorname{Tr}\left(M_{0}^{-1}\right)^{2}\right)^{2}}+\frac{10\left(\operatorname{Tr}\left(M_{0}^{-1}\right)^{3}\right)^{2}}{3\left(\operatorname{Tr}\left(M_{0}^{-1}\right)^{2}\right)^{3}}\right) .
\end{gathered}
$$

We explicitly calculate the derivative of the free energy in order $\frac{1}{m^{2}}$ to see that the free energy is independent of $r$. If we insert the known dependence 
on $q_{11,2}, q_{11,11}$ and $y_{0}$ of the terms depending themselves on $M_{0}$, we can differentiate the free energy both with respect to $r$ and $\alpha$. With $\frac{d y}{d r}=0$ and the only implicit dependence on $r$ through $\frac{d q_{11,11}^{02}}{d r}$ it follows:

$$
\begin{aligned}
& m n \frac{d \beta f_{0}}{d r}=m n \frac{\lambda^{2}}{4}\left[\left(q_{11,2}^{00}+\frac{1}{m} q_{11,2}^{01}+\frac{1}{m^{2}} q_{11,2}^{02}\right)^{2}-\left(q_{11,11}^{00}+\frac{1}{m} q_{11,11}^{01}+\frac{1}{m^{2}} q_{11,11}^{02}\right)^{2}\right. \\
&\left.+2(r-1) q_{11,2}^{00} \frac{1}{m^{2}} \frac{d q_{11,2}^{02}}{d r}-2 r q_{11,11}^{00} \frac{1}{m^{2}} \frac{d q_{11,11}^{02}}{d r}\right] \\
&+\frac{m n}{2}\left[\frac{1}{y_{00} m^{2}} \frac{d y_{02}}{d r}-\frac{1}{y_{00} m^{2} r} \frac{d y_{02}}{d r}+\frac{1}{\left(y_{00}-r \lambda^{2} \Delta q_{00}\right) m^{2} r}\left(\frac{d y_{02}}{d r}-\lambda^{2} \Delta q_{02}-r \lambda^{2} \frac{d \Delta q_{02}}{d r}\right)\right. \\
&+\frac{1}{r^{2}} \ln (y)-\frac{1}{r^{2}} \ln \left(y-r \lambda^{2} \Delta q\right)-\frac{\lambda^{2}}{\left(y_{00}-r \lambda^{2} \Delta q_{00}\right) m^{2}} \frac{d q_{11,11}^{02}}{d r} \\
&\left.+\frac{\lambda^{2} q_{11,11}^{00}}{\left(y_{00}-r \lambda^{2} \Delta q_{00}\right)^{2} m^{2}}\left(\frac{d y_{02}}{d r}-\lambda^{2} \Delta q_{02}-r \lambda^{2} \frac{d \Delta q_{02}}{d r}\right)\right] \\
&+\frac{n}{2}\left[\frac{1}{\left(y_{00}\right)^{2} r^{2}}-\frac{1}{\left(x_{2}^{00}\right)^{2} r^{2}}-\frac{2}{y_{00}^{3} m^{2}} \frac{d y_{02}}{d r}\right. \\
&\left.+\frac{2}{r y_{00}^{3} m^{2}} \frac{d y_{02}}{d r}-\frac{2}{r\left(x_{2}^{00}\right)^{3} m^{2}} \frac{d x_{2}^{02}}{d r}+\frac{2 \lambda^{2}}{m^{2}\left(x_{2}^{00}\right)^{3}} \frac{d q_{11,11}^{02}}{d r}-\frac{6 \lambda^{2} q_{11,11}^{00}}{\left(y_{00}-r \lambda^{2} \Delta q_{00}\right)^{4} m^{2}} \frac{d x_{2}^{02}}{d r}\right] \\
&-\frac{1}{m} \frac{d}{d r}\left(\frac{3 \operatorname{Tr}\left(M_{0}^{-1}\right)^{4}}{\left(\operatorname{Tr}\left(M_{0}^{-1}\right)^{2}\right)^{2}}+\frac{10\left(\operatorname{Tr}\left(M_{0}^{-1}\right)^{3}\right)^{2}}{3\left(\operatorname{Tr}\left(M_{0}^{-1}\right)^{2}\right)^{3}}\right) . \\
&(\mathrm{B.35})
\end{aligned}
$$

In second order (which is the order in which replica symmetry is broken with full-BSB) we find

$$
\begin{aligned}
\frac{d}{d r} \beta f_{02} & =\frac{\lambda^{2}}{4}\left[2 q_{11}^{00} \Delta q_{02}-2 r q_{11}^{00} \frac{d q_{11,11}^{02}}{d r}\right] \\
& +\frac{1}{2}\left[\frac{\lambda}{r} \Delta q_{02}+\frac{\lambda^{2}}{r}\left(-\frac{d q_{11,11}^{02}}{d r}-r \Delta q_{01}\right)-\lambda \frac{d q_{11,11^{02}}}{d r}\right] \\
& +[0]-\mathcal{O}_{00} \frac{d}{d r}\left(\frac{3 \operatorname{Tr}\left(M_{0}^{-1}\right)^{4}}{\left(\operatorname{Tr}\left(M_{0}^{-1}\right)^{2}\right)^{2}}+\frac{10\left(\operatorname{Tr}\left(M_{0}^{-1}\right)^{3}\right)^{2}}{3\left(\operatorname{Tr}\left(M_{0}^{-1}\right)^{2}\right)^{3}}\right) \\
& =0 .
\end{aligned}
$$

We used $y_{00}=\lambda, \Delta q_{00}=0$ and $q_{11,2}^{00}=q_{11,11}^{00}=1-\frac{1}{\lambda}$ and counted only those terms, which contribute to the regarding order, for example only the terms of order $\frac{1}{m}$ of the saddle point corrections, which are itself already of order $\frac{1}{m}$. This result does not enable us to distinguish between stability and breaking of replica symmetry. 


\section{B.7. MAXIMIZING THE FREE ENERGIES WITH RESPECT TO $R$}

\section{B.7.1 Maximizing $f_{2}$}

In order $\frac{1}{k^{2}}$ the free energy of the broken replica symmetry ansatz with bonds distributed according to the \pm -J model (where $q_{11}^{(1)}=0$ ) can be written as

$$
\begin{aligned}
& \quad \beta f_{2}^{1-R S B}=\beta f_{2}^{R S}+\frac{\lambda^{2}}{4} \sum_{\substack{\alpha, \beta \\
\mu, \nu}}\left(q_{\alpha \beta}^{\mu \nu(1)}\right)^{2}-\frac{\lambda^{4}}{8} \sum_{\substack{\alpha, \beta, \gamma, \delta \\
\mu, \nu, \gamma, \rho}} q_{\alpha \beta}^{\mu \nu(1)} q_{\gamma \delta}^{\eta \rho(1)} q_{\alpha \beta \gamma \delta}^{\mu \nu \eta \rho(0)} \\
& =\frac{\lambda^{2}}{4}\left((r-1)\left(q_{11,2}^{(1)}\right)^{2}+(n-r)\left(q_{11,11}^{(1)}\right)^{2}\right) \\
& -\frac{\lambda^{4}}{8} q_{11,2}^{(1)}\left[2(r-1) q_{11,2}^{(1)} q_{22}^{(0)}+4(r-1)(r-2) q_{11,2}^{(1)} q_{211}^{(0)}+(r-1)(r-2)(r-3) q_{11,2}^{(1)} q_{1111}^{(0)}\right. \\
& \left.+4(r-1)(n-r) q_{11,11}^{(1)} q_{211}^{(0)}+2(r-1)(r-2)(n-r) q_{11,11}^{(1)} q_{1111}^{(0)}+(r-1)(n-r)(n-2 r) q_{11,11}^{(1)} q_{1111}^{(0)}\right] \\
& -\frac{\lambda^{4}}{8} q_{11,11}^{(1)}\left[4(r-1)(n-r) q_{11,2}^{(1)} q_{211}^{(0)}+2(r-1)(r-2)(n-r) q_{11,2}^{(1)} q_{1111}^{(0)}+(r-1)(n-r)(n-2 r) q_{11,2}^{(1)} q_{1111}^{(0)}\right. \\
& +2(n-r) q_{11,11}^{(1)} q_{22}^{(0)}+4(r-1)(n-r) q_{11,11}^{(1)} q_{211}^{(0)}+4(n-r)(n-2 r) q_{11,11}^{(1)} q_{211}^{(0)}+2(r-1)^{2}(n-r) q_{11,11}^{(1)} q_{1111}^{(0)} \\
& \left.+4(r-1)(n-r)(n-2 r) q_{11,11}^{(1)} q_{1111}^{(0)}+(n-r)(n-2 r)(n-3 r) q_{11,11}^{(1)} q_{1111}^{(0)}\right] .
\end{aligned}
$$

The result in order $\frac{1}{k^{2}}$ is $(r-1) q_{11,2}^{(1)}=r q_{11,11}^{(1)}$ (see within the thesis). If we plug it into the free energy we find $\beta f_{2}^{1-R S B}=\beta f_{2}^{R S}$.

In case of Gaussian bonds we find $q_{11}^{(1)}=c(\lambda)$, with the free energy $\beta f_{2}^{R S \text {,gauss }}$. The general solution for broken replica symmetry is $(r-1) q_{11,2}^{(1)}=$ $r q_{11,11}^{(1)}-c(\lambda)$. As pointed out in the thesis, the contributions of all sums depending linearly on the first order overlaps are the same for replica symmetry and 1-RSB. When we calculate the contributions from the first line in equation B.37, we find

$$
\frac{\lambda^{2}}{4} \sum_{\substack{\alpha, \beta \\ \mu, \nu}}\left(q_{\alpha \beta}^{\mu \nu(1)}\right)^{2}-\frac{\lambda^{4}}{8} \sum_{\substack{\alpha, \beta, \gamma, \delta \\ \mu, \nu, \eta, \rho}} q_{\alpha \beta}^{\mu \nu(1)} q_{\gamma \delta}^{\eta \rho(1)} q_{\alpha \beta \gamma \delta}^{\mu \nu \eta \rho(0)}=-\frac{c(\lambda)^{2} \lambda^{2}}{2}(\lambda-1),
$$

for both ansätze. We see that the free energy is the same for the replica symmetric ansatz and the 1-RSB ansatz as it does not depend on $r$, even in case of Gaussian bonds. 


\section{Appendix C}

\section{Introducing bond chaos}

We want to derive equation 5.7 in detail. To this end, we note that $\frac{\partial}{\partial t} \log Z_{t}$ can be rewritten in terms of derivatives of $J_{i j}$ and $J_{i j}^{\prime}$ as

$$
\begin{aligned}
\frac{\partial}{\partial t} \log Z_{t} & =\frac{\partial}{\partial t} \log \operatorname{Tr} e^{-\beta \mathcal{H}_{t}} \\
& =\frac{1}{Z_{t}} \operatorname{Tr}\left(\frac{\beta}{2 \sqrt{t N}} \sum_{i<j} J_{i j}^{\prime} \vec{s}_{i} \vec{s}_{j}-\frac{\beta}{2 \sqrt{(1-t) N}} \sum_{i<j} J_{i j} \vec{s}_{i} \vec{s}_{j}\right) \\
& =\frac{1}{2 t} \sum_{i<j} J_{i j}^{\prime} \frac{\partial}{\partial J_{i j}^{\prime}} \log Z_{t}-\frac{1}{2(1-t)} \sum_{i<j} J_{i j} \frac{\partial}{\partial J_{i j}} \log Z_{t},
\end{aligned}
$$

and analoguously for $\frac{\partial}{\partial \tau} \log Z_{\tau}^{\prime}$. This gives

$$
\begin{aligned}
& E_{J} \frac{\partial}{\partial t} \log Z_{t} \frac{\partial}{\partial \tau} \log Z_{\tau}^{\prime} \\
& E_{J} \sum_{i<j, k<l}\left(\frac{1}{2 t} \sum_{i<j} J_{i j}^{\prime} \frac{\partial}{\partial J_{i j}^{\prime}} \log Z_{t}-\frac{1}{2(1-t)} \sum_{i<j} J_{i j} \frac{\partial}{\partial J_{i j}} \log Z_{t}\right) \\
& \quad \times\left(\frac{1}{2 \tau} \sum_{i<j} J_{k l}^{\prime \prime} \frac{\partial}{\partial J_{k l}^{\prime \prime}} \log Z_{\tau}-\frac{1}{2(1-\tau)} \sum_{i<j} J_{k l} \frac{\partial}{\partial J_{k l}} \log Z_{\tau}\right) .
\end{aligned}
$$

We can further manipulate the right hand side by an integration by parts (with the short hand notation of $o$ for the corresponding terms of equa- 
tion (C.2) ) by making use of the independence of the different sets of bonds

$$
\begin{aligned}
E_{J} J_{i j}^{\prime} J_{k l}^{\prime \prime} O & =\int \cdots d J_{i j}^{\prime} e^{-J_{i j}^{\prime}{ }^{2} / 2} J_{i j}^{\prime} J_{k l}^{\prime \prime} O \\
& =\int \cdots d J_{i j}^{\prime} e^{-J_{i j}^{\prime}{ }^{2} / 2} \frac{\partial}{\partial J_{i j}^{\prime}} J_{k l}^{\prime \prime} O \\
& =E_{J} \frac{\partial}{\partial J_{i j}^{\prime}} J_{k l}^{\prime \prime} O
\end{aligned}
$$

followed by a second integration by parts for the second integration variable

$$
\begin{aligned}
E_{J} J_{k l}^{\prime \prime} \frac{\partial}{\partial J_{i j}^{\prime}} o & =\int \cdots d J_{k l}^{\prime \prime} e^{-J_{k l}^{\prime \prime 2} / 2} J_{k l}^{\prime \prime} \frac{\partial}{\partial J_{i j}^{\prime}} o \\
& =E_{J} \frac{\partial}{\partial J_{k l}^{\prime \prime}} \frac{\partial}{\partial J_{i j}^{\prime}} o .
\end{aligned}
$$

In case of the term $E_{J} J_{i j} J_{k l} O$ we get

$$
E_{J} J_{i j} J_{k l} O=E_{J}\left(\delta_{(i j),(k l)}+\frac{\partial}{\partial J_{k l}} \frac{\partial}{\partial J_{i j}} o\right)
$$

With these manipulations equation $\mathrm{C.2}$ is

$$
\begin{gathered}
E_{J} \frac{\partial}{\partial t} \log Z_{t} \frac{\partial}{\partial \tau} \log Z_{\tau}^{\prime}=E_{J} \sum_{i<j, k<l}\left[\frac{1}{4 t \tau} \frac{\partial}{\partial J_{k l}^{\prime \prime}} \frac{\partial}{\partial J_{i j}^{\prime}} \frac{\partial \log Z_{\tau}^{\prime}}{\partial J_{k l}^{\prime \prime}} \frac{\partial \log Z_{t}}{\partial J_{i j}^{\prime}}\right. \\
-\frac{1}{4 t(1-\tau)} \frac{\partial}{\partial J_{k l}} \frac{\partial}{\partial J_{i j}^{\prime}} \frac{\partial \log Z_{\tau}^{\prime}}{\partial J_{k l}} \frac{\partial \log Z_{t}}{\partial J_{i j}^{\prime}}-\frac{1}{4(1-t) \tau} \frac{\partial}{\partial J_{k l}^{\prime \prime}} \frac{\partial}{\partial J_{i j}} \frac{\partial \log Z_{\tau}^{\prime}}{\partial J_{k l}^{\prime \prime}} \frac{\partial \log Z_{t}}{\partial J_{i j}} \\
\left.+\frac{1}{4(1-t)(1-\tau)} \frac{\partial}{\partial J_{k l}} \frac{\partial}{\partial J_{i j}} \frac{\partial \log Z_{\tau}^{\prime}}{\partial J_{k l}} \frac{\partial \log Z_{t}}{\partial J_{i j}}\right]+E_{J} \sum_{i<j} \frac{\partial \log Z_{\tau}^{\prime}}{\partial J_{i j}} \frac{\partial \log Z_{t}}{\partial J_{i j}}
\end{gathered}
$$

In the last term of the penultimate line the links $J_{i j}$ and $J_{k l}$ are different, since the case $(i j)=(k l)$ is represented in the last line. The single terms of the right hand side are nothing but thermal averages of the link between two 
spins. To see this, we present this transformation for two instances:

$$
\begin{aligned}
\frac{\partial \log Z_{t}}{\partial J_{i j}} & =\frac{\partial \log \operatorname{Tr} e^{-\beta \mathcal{H}_{t}}}{\partial J_{i j}}=\frac{\beta \sqrt{\frac{1-t}{N}} \operatorname{Tr}\left(\vec{s}_{i} \vec{s}_{j}\right) e^{-\beta \mathcal{H}_{t}}}{\operatorname{Tr} e^{-\beta \mathcal{H}_{t}}}=\beta \sqrt{\frac{1-t}{N}}\left\langle\vec{s}_{i} \vec{s}_{j}\right\rangle_{t} \\
\frac{\partial^{2} \log Z_{t}}{\partial J_{i j} \partial J_{k l}^{\prime}} & =\frac{\partial}{\partial J_{k l}^{\prime}} \frac{\beta \sqrt{\frac{1-t}{N}} \operatorname{Tr}\left(\vec{s}_{i} \vec{s}_{j}\right) e^{-\beta \mathcal{H}_{t}}}{\operatorname{Tr} e^{-\beta \mathcal{H}_{t}}} \\
= & \beta \sqrt{\frac{1-t}{N}} \beta \sqrt{\frac{t}{N}} \frac{\operatorname{Tr}\left(\vec{s}_{i} \vec{s}_{j}\right)\left(\vec{s}_{k} \vec{s}_{l}\right) e^{-\beta \mathcal{H}_{t}} \operatorname{Tr} e^{-\beta \mathcal{H}_{t}}-\operatorname{Tr}\left(\vec{s}_{i} \vec{s}_{j}\right) e^{-\beta \mathcal{H}_{t}} \operatorname{Tr}\left(\vec{s}_{k} \vec{s}_{l}\right) e^{-\beta \mathcal{H}_{t}}}{\left(\operatorname{Tr} e^{-\beta \mathcal{H}_{t}}\right)^{2}} \\
= & \frac{\beta^{2}}{N} \sqrt{(1-t) t}\left(\left\langle\left(\vec{s}_{i} \vec{s}_{j}\right)\left(\vec{s}_{k} \vec{s}_{l}\right)\right\rangle_{t}-\left\langle\left(\vec{s}_{i} \vec{s}_{j}\right)\right\rangle_{t}\left\langle\left(\vec{s}_{k} \vec{s}_{l}\right)\right\rangle_{t}\right)
\end{aligned}
$$

Each derivative with respect to $J_{i j}, J_{i j}^{\prime}$ or $J_{i j}^{\prime \prime}$ generates spin averages taken with respect to the interpolation parameter $t$ or $\tau$ and further gives a prefactor of $\beta \sqrt{\frac{1-t}{N}}, \beta \sqrt{\frac{t}{N}}, \beta \sqrt{\frac{1-\tau}{N}}$ or $\beta \sqrt{\frac{\tau}{N}}$ (depending on the partition function $Z_{t}$ or $Z_{\tau}^{\prime}$ the derivative is taken of). In principle, we have to take not only two, but up to four derivatives in each term of equation (C.6). Fortunately, terms with three and four derivatives cancel each other (we omit this lengthy calculation), such that $E_{J} \frac{\partial}{\partial t} \log Z_{t} \frac{\partial}{\partial \tau} \log Z_{\tau}^{\prime}$ becomes

$$
\begin{aligned}
E_{J} \frac{\partial}{\partial t} \log Z_{t} \frac{\partial}{\partial \tau} \log Z_{\tau}^{\prime} & =\frac{\beta^{2}}{4 N^{2}} E_{J} \sum_{i<j, k<l}\left(\left\langle\left(\vec{s}_{i} \vec{s}_{j}\right)\left(\vec{s}_{k} \vec{s}_{l}\right)\right\rangle_{t}-\left\langle\left(\vec{s}_{i} \vec{s}_{j}\right)\right\rangle_{t}\left\langle\left(\vec{s}_{k} \vec{s}_{l}\right)\right\rangle_{t}\right) \\
& \times\left(\left\langle\left(\vec{s}_{i} \vec{s}_{j}\right)\left(\vec{s}_{k} \vec{s}_{l}\right)\right\rangle_{\tau}-\left\langle\left(\vec{s}_{i} \vec{s}_{j}\right)\right\rangle_{\tau}\left\langle\left(\vec{s}_{k} \vec{s}_{l}\right)\right\rangle_{\tau}\right) \\
& \left.+\frac{\beta^{2}}{4 N^{2}} \frac{1}{\sqrt{1-t} \sqrt{1-\tau}} E_{J} \sum_{i<j}\left\langle\left(\vec{s}_{i} \vec{s}_{j}\right)\right\rangle_{t}\left\langle\left(\vec{s}_{i} \vec{s}_{j}\right)\right\rangle_{\tau}, \quad \text { C. } 8\right)
\end{aligned}
$$

which is equal to equation (5.7), when changing the indices of the sum to $i, j, k, l$.

Additionally to the derivation of equation (5.7) we want to describe the manipulations leading to equation (5.9), in which the spin averages have been expressed by link overlaps. There, we shift the thermal averaging over partition functions with interpolating parameters $t$ and $\tau$ to a single common symbol $(\langle\cdot\rangle)$ and introduce four different replicas, labelled by numbers 1 to 4 and the interpolating parameter according to which they are averaged, where the replicas 1 and 2 have interpolating parameter $t$ and replica 3 and 4 have parameter $\tau$. For instance, for $\left\langle\left(\vec{s}_{i} \vec{s}_{j}\right)\left(\vec{s}_{k} \vec{s}_{l}\right)\right\rangle_{t}$ we write $\left\langle\left(\vec{s}_{i}^{1 t}{\overrightarrow{s_{j}}}^{1 t}\right)\left({\overrightarrow{s_{k}}}^{1 t}{\overrightarrow{s_{l}}}^{1 t}\right)\right\rangle$ (as both links are averaged commonly with respect to the same parameter $t$ ) and for $\left\langle\left(\vec{s}_{i} \vec{s}_{j}\right)\right\rangle_{t}\left\langle\left(\vec{s}_{k} \vec{s}_{l}\right)\right\rangle_{t}$ we write $\left\langle\left({\overrightarrow{s_{i}}}^{1 t}{\overrightarrow{s_{j}}}^{1 t}\right)\left({\overrightarrow{s_{k}}}^{2 t}{\overrightarrow{s_{l}}}^{2 t}\right)\right\rangle$ (as both links are averaged 
separately but with the same parameter $t)$. Equation (5.7) then becomes

$$
\begin{gathered}
E_{J} \frac{\partial}{\partial t} \log Z_{t} \frac{\partial}{\partial \tau} \log Z_{\tau}^{\prime}=\frac{\beta^{4}}{16 N^{2}} E_{J} \sum_{i j k l}\left(\left\langle\left({\overrightarrow{s_{i}}}^{1 t}{\overrightarrow{s_{j}}}^{1 t}\right)\left({\overrightarrow{s_{k}}}^{1 t}{\overrightarrow{s_{l}}}^{1 t}\right)\left({\overrightarrow{s_{i}}}^{3 \tau}{\overrightarrow{s_{j}}}^{3 \tau}\right)\left({\overrightarrow{s_{k}}}^{3 \tau}{\overrightarrow{s_{l}}}^{3 \tau}\right)\right\rangle\right. \\
-\left\langle\left({\overrightarrow{s_{i}}}^{1 t}{\overrightarrow{s_{j}}}^{1 t}\right)\left({\overrightarrow{s_{k}}}^{2 t}{\overrightarrow{s_{l}}}^{2 t}\right)\left({\overrightarrow{s_{i}}}^{3 \tau}{\overrightarrow{s_{j}}}^{3 \tau}\right)\left({\overrightarrow{s_{k}}}^{3 \tau}{\overrightarrow{s_{l}}}^{3 \tau}\right)\right\rangle \\
\left.-\left\langle\left({\overrightarrow{s_{i}}}^{1 t}{\overrightarrow{s_{j}}}^{1 t}\right)\left({\overrightarrow{s_{k}}}^{1 t}{\overrightarrow{s_{l}}}^{1 t}\right)\left({\overrightarrow{s_{i}}}^{4 \tau}{\overrightarrow{s_{j}}}^{4 \tau}\right)\left({\overrightarrow{s_{k}}}^{3 \tau}{\overrightarrow{s_{l}}}^{3 \tau}\right)\right\rangle+\left\langle\left({\overrightarrow{s_{i}}}^{1 t}{\overrightarrow{s_{j}}}^{1 t}\right)\left({\overrightarrow{s_{k}}}^{2 t}{\overrightarrow{s_{l}}}^{2 t}\right)\left({\overrightarrow{s_{i}}}^{4 \tau}{\overrightarrow{s_{j}}}^{4 \tau}\right)\left({\overrightarrow{s_{k}}}^{3 \tau}{\overrightarrow{s_{l}}}^{3 \tau}\right)\right\rangle\right) \\
+\frac{\beta^{2}}{8 N} \frac{1}{\sqrt{1-t} \sqrt{1-\tau}} E_{J} \sum_{i j}\left(\left\langle\left({\overrightarrow{s_{i}}}^{1 t}{\overrightarrow{s_{j}}}^{1 t}\right)\left({\overrightarrow{s_{i}}}^{3 \tau}{\overrightarrow{s_{j}}}^{3 \tau}\right)\right\rangle-m^{2} N\right) \quad \text { (C.9) }
\end{gathered}
$$

Now we introduce the link overlap as, for instance in case of the two replicas 1 and $3, q_{L}^{13}=\frac{2}{N(N-1)} \sum_{i<j}\left\langle\vec{s}_{i} \vec{s}_{j}\right\rangle_{t}\left\langle\vec{s}_{i} \vec{s}_{j}\right\rangle_{\tau}=\frac{1}{N(N-1)}\left(\sum_{i j}\left\langle\left(\vec{s}_{i}^{1 t} \vec{s}_{j}^{1 t}\right)\left(\vec{s}_{i}^{3 \tau}{\overrightarrow{s_{j}}}^{3 \tau}\right)\right\rangle-\right.$ $\left.m^{2} N\right)$. Expressed in terms of link overlaps and separating the sum over $i j k l$, a single term in equation (C.9) as $\sum_{i j k l}\left({\overrightarrow{s_{i}}}^{1 t}{\overrightarrow{s_{j}}}^{1 t}\right)\left({\overrightarrow{s_{k}}}^{1 t}{\overrightarrow{s_{l}}}^{1 t}\right)\left({\overrightarrow{s_{i}}}^{4 \tau}{\overrightarrow{s_{j}}}^{4 \tau}\right)\left({\overrightarrow{s_{k}}}^{3 \tau}{\overrightarrow{s_{l}}}^{3 \tau}\right)=$ $\sum_{i j}\left(\vec{s}_{i}^{1 t}{\overrightarrow{s_{j}}}^{1 t}\right)\left({\overrightarrow{s_{i}}}^{4 \tau}{\overrightarrow{s_{j}}}^{4 \tau}\right) \sum_{k l}\left({\overrightarrow{s_{k}}}^{1 t}{\overrightarrow{s_{l}}}^{1 t}\right)\left({\overrightarrow{s_{k}}}^{3 \tau}{\overrightarrow{s_{l}}}^{3 \tau}\right)$ becomes $N^{2}(N-1)^{2}\left(q_{L}^{14}+N m^{2}\right)\left(q_{L}^{13}+\right.$ $N m^{2}$ ). When we insert these transformations into equation (C.9) the contributions involving the term $\mathrm{Nm}^{2}$ cancel and we find

$$
\begin{aligned}
E_{J} \frac{\partial}{\partial t} \log Z_{t} \frac{\partial}{\partial \tau} \log Z_{\tau}^{\prime} & =\frac{\beta^{4}(N-1)^{2}}{16} E_{J}\left\langle\left(q_{L}^{13}\right)^{2}-q_{L}^{13} q_{L}^{23}-q_{L}^{14} q_{L}^{13}+q_{L}^{14} q_{L}^{23}\right\rangle \\
& +\frac{\beta^{2}(N-1)}{8} \frac{1}{\sqrt{1-t} \sqrt{1-\tau}} E_{J}\left\langle q_{L}^{13}\right\rangle
\end{aligned}
$$

Assuming replica symmetry in the large- $m$ limit, we end up with equation (5.9). 


\section{Appendix D}

\section{Replica calculation for two constrained copies}

In this appendix we present the basic steps of the calculation of the $n$-times replicated partition function of two copies of the same spin glass (with possibly different sets of bonds), which are constrained to have a mutual link overlap $q_{L}$. The $n$-times replicated partition function of this system is

$$
\begin{aligned}
& E_{J} Z_{\epsilon, J}^{n}\left(q_{L}\right)=E_{J} \operatorname{Tr}_{\vec{s}_{\alpha}} \prod_{\alpha=1}^{n}\left(\delta\left(q_{L}-\frac{1}{N(N-1)} \sum_{\mu \nu}\left(\sum_{i} s_{i \mu, \alpha}^{(10)} s_{i \nu, \alpha}^{(3 \epsilon)}\right)^{2}-\frac{m^{2}}{N-1}\right)\right) \\
& \times \exp \left(\frac{\beta}{\sqrt{N}} \sum_{\alpha} \sum_{i<j} K_{i j}(0) \vec{s}_{i \alpha}^{(10)} \vec{s}_{j \alpha}^{(10)}+\frac{\beta}{\sqrt{N}} \sum_{\alpha} \sum_{i<j} K_{i j}(\epsilon) \vec{s}_{i \alpha}^{(3 \epsilon)} \vec{s}_{j \alpha}^{(3 \epsilon)}\right) . \quad(\mathrm{D} .1)
\end{aligned}
$$

First we integrate over the disorder, i.e. over the basic independent sets of bonds of $K_{i j}(\epsilon), K_{i j}^{0}$ and $K_{i j}^{\prime}$ from equation (5.10), which we take be Gaussian with unit variance. The Gaussian integrals yield (omitting irrelevant prefactors)

$$
\begin{aligned}
& E_{J} Z_{\epsilon, J}^{n}\left(q_{L}\right) \propto \operatorname{Tr}_{\vec{s}_{\alpha}} \prod_{\alpha=1}^{n}\left(\delta\left(q_{L}-\frac{1}{N(N-1)} \sum_{\mu \nu}\left(\sum_{i} s_{i \mu, \alpha}^{(10)} s_{i \nu, \alpha}^{(3 \epsilon)}\right)^{2}-\frac{m^{2}}{N-1}\right)\right) \\
& \times \exp \left(\frac{\beta^{2}}{N} \sum_{\langle i j\rangle}\left[\left(\vec{s}_{i \alpha}^{(10)} \vec{s}_{j \alpha}^{(10)}+\frac{1}{\sqrt{1+\epsilon^{2}}} \vec{s}_{i \alpha}^{(3 \epsilon)} \vec{s}_{j \alpha}^{(3 \epsilon)}\right)^{2}+\left(\frac{1}{\sqrt{1+\epsilon^{2}}} \vec{s}_{i \alpha}^{(3 \epsilon)} \vec{s}_{j \alpha}^{(3 \epsilon)}\right)^{2}\right]\right) .
\end{aligned}
$$

We prepare a Hubbard-Stratonovich transformation by reordering the sums over the spins (with $\mu, \nu$ denoting the spin components and $\alpha, \beta$ the replica 
indices) and get

$$
\begin{gathered}
E_{J} Z_{\epsilon, J}^{n}\left(q_{L}\right) \propto \operatorname{Tr}_{\vec{s}_{\alpha}} \prod_{\alpha=1}^{n}\left(\delta\left(q_{L}-\frac{1}{N(N-1)} \sum_{\mu \nu}\left(\sum_{i} s_{i \mu, \alpha}^{(10)} s_{i \nu, \alpha}^{(3 \epsilon)}\right)^{2}-\frac{m^{2}}{N-1}\right)\right) \\
\times \exp \left(\frac { \beta ^ { 2 } } { 4 N } \left[\sum_{\substack{\alpha, \beta \\
\mu, \nu}}\left(\sum_{i} s_{i \mu, \alpha}^{(10)} s_{i \nu, \beta}^{(10)}\right)^{2}+\frac{2}{\sqrt{1+\epsilon^{2}}} \sum_{\substack{\alpha, \beta \\
\mu, \nu}}\left(\sum_{i} s_{i \mu, \alpha}^{(10)} s_{i \nu, \beta}^{(3 \epsilon)}\right)^{2}\right.\right. \\
\left.\left.\left.+\frac{1+\epsilon^{2}}{1+\epsilon^{2}} \sum_{\substack{\alpha, \beta \\
\mu, \nu}}\left(\sum_{i} s_{i \mu, \alpha}^{(3 \epsilon)} s_{i \nu, \beta}^{(3 \epsilon)}\right)^{2}\right]\right) . \quad \text { D. } 3\right)
\end{gathered}
$$

We write the $\delta$-constraint in its integral representation with the parameters $z_{\alpha}^{\prime}$ as $\prod_{\alpha=1}^{n}\left(\delta\left(q_{L}-\frac{1}{N(N-1)} \sum_{\mu \nu}\left(\sum_{i} s_{i \mu, \alpha}^{(10)} s_{i \nu, \alpha}^{(3 \epsilon)}\right)^{2}-\frac{m^{2}}{N-1}\right)\right)=\int_{-i \infty}^{i \infty}\left(\prod_{\alpha} \frac{d z_{\alpha}^{\prime}}{2 \pi}\right) \exp (-$ $\left.q_{L} \sum_{\alpha} z_{\alpha}^{\prime}+\sum_{\alpha, \mu, \nu} z_{\alpha}^{\prime} \frac{1}{N(N-1)}\left(\sum_{i} s_{i \mu, \alpha}^{(10)} s_{i \nu, \alpha}^{(3 \epsilon)}\right)^{2}-\sum_{\alpha} z_{\alpha}^{\prime} \frac{m^{2}}{N-1}\right)$. With the substitution $z_{\alpha}=\frac{z_{\alpha}^{\prime}}{N-1}$ we find

$$
\begin{gathered}
E_{J} Z_{\epsilon, J}^{n}\left(q_{L}\right) \propto \int\left(\prod_{\alpha} \frac{(N-1) d z_{\alpha}}{2 \pi}\right) e^{-(N-1) \sum_{\alpha} q_{L} z_{\alpha}-m^{2} \sum_{\alpha} z_{\alpha}} \\
\times \operatorname{Tr}_{\vec{s}_{\alpha}} \exp \left(\frac { \beta ^ { 2 } } { 4 N } \left[\sum_{\substack{\alpha, \beta \\
\mu, \nu}}\left(\sum_{i} s_{i \mu, \alpha}^{(10)} s_{i \nu, \beta}^{(10)}\right)^{2}+\sum_{\alpha, \mu, \nu}\left(\frac{4}{\beta^{2}} z_{\alpha}+\frac{2}{\sqrt{1+\epsilon^{2}}}\right)\left(\sum_{i} s_{i \mu, \alpha}^{(10)} s_{i \nu, \beta}^{(3 \epsilon)}\right)^{2}\right.\right. \\
\left.\left.+\frac{2}{\sqrt{1+\epsilon^{2}}} \sum_{\substack{\alpha \neq \beta \\
\mu, \nu}}\left(\sum_{i} s_{i \mu, \alpha}^{(10)} s_{i \nu, \beta}^{(3 \epsilon)}\right)^{2}+\sum_{\substack{\alpha, \beta \\
\mu, \nu}}\left(\sum_{i} s_{i \mu, \alpha}^{(3 \epsilon)} s_{i \nu, \beta}^{(3 \epsilon)}\right)^{2}\right]\right) . \quad \text { (D.4) }
\end{gathered}
$$

In the next step we introduce the new integration variables $Q_{\alpha \beta}^{\mu \nu(10)}, Q_{\alpha \beta}^{\mu \nu(3 \epsilon)}$ and $R_{\alpha \beta}^{\mu \nu}$ by the Hubbard-Stratonovich transformation, which reduces the quadratic dependence on spin component products as $\left(\sum_{i} s_{i \mu, \alpha}^{(10)} s_{i \nu, \beta}^{(10)}\right)^{2}$ to a linear dependence. For instance, the transformation yields

$$
\begin{aligned}
e^{\frac{\beta^{2}}{2 N} \sum_{\substack{\alpha<\beta \\
\mu, \nu}}\left(\sum_{i} s_{i \mu, \alpha}^{(10)} s_{i \nu, \beta}^{(10)}\right)^{2}}=\int\left(\prod_{\substack{\alpha, \beta \\
\mu, \nu}} Q_{\alpha \beta}^{\mu \nu}\right) e^{-\frac{N}{2 \beta^{2}} \sum_{\substack{\alpha<\beta \\
\mu, \nu}}\left(Q_{\alpha \beta}^{\mu \nu}\right)^{2}+\sum_{\substack{\alpha<\beta \\
\mu, \nu}} Q_{\alpha \beta}^{\mu \nu} \sum_{i} s_{i \mu, \alpha}^{(10)} s_{i \nu, \beta}^{(10)}} \\
e^{\frac{\beta^{2}}{2 N} \sum_{\alpha, \mu, \nu}\left(\sum_{i} s_{i \mu, \alpha}^{(10)} s_{i \nu, \alpha}^{(2 \epsilon)}\right)^{2}}=\int\left(\prod_{\alpha, \mu, \nu} R_{\alpha \alpha}^{\mu \nu}\right) e^{-\frac{N}{2 \beta^{2}} \sum_{\alpha, \mu, \nu}\left(\frac{2}{\beta^{2}} z_{\alpha}+\frac{1}{\sqrt{1+\epsilon^{2}}}\right)^{-1}\left(R_{\alpha \alpha}^{\mu \nu}\right)^{2}+\sum_{\alpha, \mu, \nu} R_{\alpha \alpha}^{\mu \nu} \sum_{i} s_{i \mu, \alpha}^{(10)} s_{i \nu, \alpha}^{(2 \epsilon)}}
\end{aligned}
$$

and analoguously for $Q_{\alpha \beta}^{\mu \nu(3 \epsilon)}$ and $R_{\alpha \beta}^{\mu \nu}$ with $\alpha \neq \beta$. Furthermore we split the tensor $Q_{\alpha \beta}^{\mu \nu}$ into its diagonal and off-diagonal part $\sum_{\substack{\alpha, \beta \\ \mu, \nu}} Q_{\alpha \beta}^{\mu \nu}=2 \sum_{\substack{\alpha<\beta \\ \mu, \nu}} Q_{\alpha \beta}^{\mu \nu}+$ 
$2 \sum_{\alpha, \mu<\nu} T_{\alpha}^{\mu \nu}+\sum_{\alpha, \mu}\left(Q_{\alpha \alpha}+T_{\alpha}^{\mu \mu}\right)$ (for both $Q^{(10)}$ and $\left.Q^{(3 \epsilon)}\right)$. There, $T_{\alpha}^{\mu \nu}$ is a traceless tensor, i.e. $\sum_{\mu} T_{\alpha}^{\mu \mu}=0$. Now, the Trace over the spins can be carried out. To this end we note that the sum over the sites $i=1 \cdots N$ in the exponential, which includes the dependence of the spins (denoted as the external exponent), in fact gives $N$ times the same integral. Therefore, we expand this integral into its power series and write the constraint $\delta(m-$ $\vec{s}^{2}$ ) in its integral representation, which includes the term $e^{-z \vec{s}^{2}}$. We see that evaluating the Trace reduces to solving Gaussian integrals and only the combinations including quadratic terms in $s_{\alpha \mu}^{(10)}$ and $s_{\alpha \mu}^{(2 \epsilon)}$ survive. However, we find it more appropriate to carry out the Trace using spherical coordinates. For the various cases (with $x, y, z$ denoting different spins and $i, j, k$ denoting different components) we get

$$
\begin{aligned}
\left(x_{i}\right)^{2}\left(y_{j}\right)^{2} & =S_{m}^{2 n} \\
\left(x_{i}\right)^{2}\left(x_{j}\right)^{2} & =\frac{m}{m+2} S_{m}^{2 n} \\
\left(x_{i}\right)^{2}\left(y_{j}\right)^{2}\left(z_{k}\right)^{2} & =S_{m}^{2 n} \\
\left(x_{i}\right)^{2}\left(x_{j}\right)^{2}\left(y_{k}\right)^{2} & =\frac{m}{m+2} S_{m}^{2 n} \\
\left(x_{i}\right)^{2}\left(x_{j}\right)^{2}\left(x_{k}\right)^{2} & =\frac{m^{2}}{(m+2)(m+4)} S_{m}^{2 n} \\
\left(x_{i}\right)^{4}\left(y_{j}\right)^{2} & =\frac{3 m}{m+2} S_{m}^{2 n}
\end{aligned}
$$

where $S_{m}=\frac{2 \sqrt{\pi}^{m} \sqrt{m}^{m-1}}{\Gamma\left(\frac{m}{2}\right)}$ is the surface of the $m$-dimensional sphere with radius $\sqrt{m}$. Afterwards we reexponentiate the resulting terms in $Q_{\alpha \beta}^{\mu \nu(10)}$, $Q_{\alpha \beta}^{\mu \nu(3 \epsilon)}$ and $R_{\alpha \beta}^{\mu \nu}$. Together with the first part exponential of the HubbardStratonovich transformation, which was independent of evaluating the Trace over the spins, we finally end up in equation (5.15). 


\section{List of Figures}

1.1 Example of a frustrated loop of four Ising spins. . . . . . . . . 3

1.2 Distribution of the overlap in three different cases. Left: Replica symmetry, a single overlap at $q_{E A}$; Middle: 2-RSB, three different overlaps are possible; Right: Full RSB, the distribution has non-vanishing support between 0 and $q_{E A}$. . . . . . . . . 10

1.3 Spin configurations on frustrated plaquettes. The signs inside the plaquettes denote the sign of the local chirality. The different configurations can be generated from each other by a global reflection of the spins, but not by a global rotation. . . 15

2.1 Two examples of six spins on a Bethe lattice with fixed connectivity of three. . . . . . . . . . . 23

2.2 Left: The three spins $\vec{s}_{1}, \vec{s}_{2}$ and $\vec{s}_{3}$ and their back branches are merged onto spin $\vec{s}_{0}$ with coupling constants $J_{1}, J_{2}$ and $J_{3}$ in this example with $k=3$. In the next step of the iteration, $\vec{s}_{0}$ and two other branches will be merged onto the spin $\vec{s}_{4}$. Right: In contrast, the true local field $\vec{H}_{0}$ onto the spin $\vec{s}_{0}$ is the sum of the interaction with all $k+1$, here 4 , of its neighbours. See text for further explanation. . . . . . . . . . . . . . . . . 24

2.3 Results for 96 spins with 8 components on a Bethe lattice with connectivity equal to eight. The order parameter is plotted against the temperature for four different Bethe lattices. When $(\Delta h)^{2}$ becomes larger than zero, the phase transition happens. . . . . . . . . . . . . . . . . 38

2.4 Critical scaling of the order parameter for 96 spins with 8 components on a Bethe lattice with connectivity equal to eight. The critical exponent is close to the 1 , the exponent of the Ising universality class. . . . . . . . . . . . . . . . . . . . . . 39

2.5 Phase transition for 256 spins with 8 components on four different Bethe lattices with connectivity equal to 4. . . . . . . . 39 
2.6 Critical scaling of the order parameter for 256 spins with 8 components on a Bethe lattice with connectivity equal 4. The critical exponent is comparable to that of the Ising universality class. . . . . . . . . . . . . . . . . 40

2.7 Phase transition for 256 spins with 8 components on four different Bethe lattices with connectivity equal to 8. . . . . . . . 40

2.8 Critical scaling of the order parameter for 256 spins with 8 components on a Bethe lattice with connectivity equal 8. The critical exponent is close to 1. . . . . . . . . . . . . 41

2.9 Phase transition for 512 spins with 4 components on four different Bethe lattices with connectivity equal to 6. . . . . . . . 41

2.10 Critical scaling of the order parameter for 512 spins with 4 components on a Bethe lattice with connectivity equal 6 . The critical exponent is close to 1. . . . . . . . . . . . . . 42

2.11 Histogram of the probability distribution of the link overlap for the four lowest temperatures simulated. . . . . . . . . . . . 49

2.12 Histogram of the probability distribution of the link overlap for four temperatures covering a range including $T_{c} . \quad \ldots$. . . . 50

2.13 Histogram of the probability distribution of the link overlap for four temperatures above $T_{c} . \ldots \ldots \ldots \ldots$

2.14 Histogram of the probability distribution of the link overlap for four temperatures above $T_{c} . \ldots \ldots \ldots \ldots \ldots$

2.15 Histogram of the probability distribution of the link overlap for the four highest temperatures. . . . . . . . . . . . 51

2.16 Scaling plot of the order parameter $q^{2}$ for a connectivity of eight and six components. See text for explanation. . . . . . . 53

2.17 Double logarithmic scaling plot of $q^{2}$ in the low temperature phase with $k+1=8$ and $m=6$. The scaling function matches a scaling of $|x|^{\gamma^{\prime}}$ with $\gamma^{\prime} \approx 1.1$ (additionally plotted). . . . . . . 54

2.18 Scaling plot of $q^{2}$, with $k+1=8$ and $m=4$. . . . . . . . . . 54

2.19 Double logarithmic scaling plot of $q^{2}$ with $k+1=8$ and $m=4$. Additionally plotted: $|x|^{1.1} \ldots \ldots \ldots \ldots \ldots \ldots 5$

2.20 Scaling plot of $q^{2}$, with $k+1=8$ and $m=3$. . . . . . . . . . 55

2.21 Double logarithmic scaling plot of $q^{2}$ with $k+1=8$ and $m=3$. Additionally plotted: $|x|^{1.1} \ldots \ldots \ldots \ldots \ldots 6$

2.22 Scaling plot of $q^{2}$, with $k+1=8$ and $m=2$. . . . . . . . . . 56

2.23 Double logarithmic scaling plot of $q^{2}$ with $k+1=8$ and $m=2$. Additionally plotted: $|x|^{1.1} \ldots \ldots \ldots \ldots \ldots . \ldots \ldots$

2.24 Two neighbouring spins $s_{i}$ and $s_{j}$ connected by $J_{i j}$ and the cavity fields onto them (indicated by the rectangular loops), not taking into account $J_{i j} \ldots \ldots \ldots \ldots \ldots$ 


\section{LIST OF FIGURES}

3.1 Downward shift of the semicircular eigenvalue density of the fully connected $m$-component spin glass: $m_{0}$ of the smallest eigenvalues (left, shaded) become null eigenvalues (deltapeak,

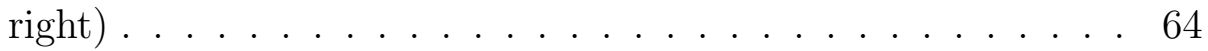

3.2 Ground state energy per spin and component for $k+1=8$ as a function of $N^{-y}$ with $y$ calculated from the scaling relation equation (3.3). The straight line extrapolates to the ground state energy $e_{0}$ in the limit $N \rightarrow \infty$. . . . . . . . . . . 66

3.3 Ground state energy per spin and component for the fully connected model. The straight line has slope $-2 / 5$. The error bars are smaller than the point size. . . . . . . . . . . 67

3.4 Eigenvalue spectra for four different Bethe lattices of 64 spins with $k+1=8$. Different colours represent different temperatures, decreasing from blue to green to pink. The larger circle has a radius of one. When the first eigenvalue crosses this circle, the phase transition happens. We see that some lattices have large real eigenvalues (lower left and upper right) and some have solely complex eigenvalues (lower right and upper

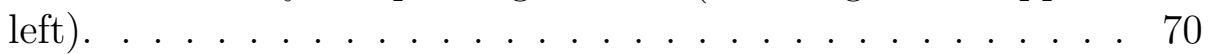

3.5 Effective number of components $m_{\mathrm{eff}}(T)(+)$ for 256 spins with 8 components on different Bethe lattices with $k+1=8$. Ad\begin{tabular}{ll|l|l|l}
\hline ditionally plotted is the order parameter from section & 2.3 & 72
\end{tabular}

3.6 Effective number of components $m_{\text {eff }}(T)(+)$ and the order parameter from section $2.3 \mid(\times)$ for 96 spins with 8 components

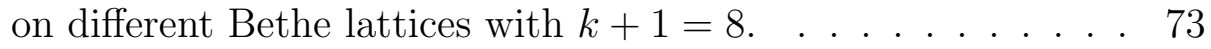

3.7 Effective number of components $m_{\text {eff }}(T)(+)$ and the order parameter from section $2.3 \mid(\times)$ for 256 spins with 8 components on different Bethe lattices with $k+1=4.6$. . . . . . . . . 73

3.8 Effective number of components $m_{\text {eff }}(T)(+)$ and the order pa-

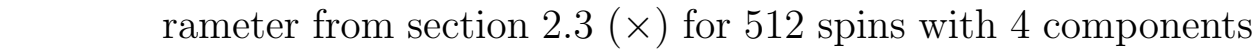

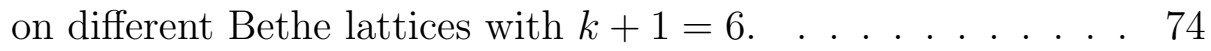

3.9 Three-dimensional plot of a configuration of local fields (128 spins with three components) in the low temperature phase before (blue) and after (pink) a single iteration step. Each configuration is only two-dimensional for this lattice at the chosen temperature, i.e. the large- $m$ limit holds. . . . . . . . . 75

3.10 Different view at plot 3.9 . The rotation of the iteration step takes place in the two-dimensional plane each configuration spans. . . . . . . . . . . . . . . . . . . . 75

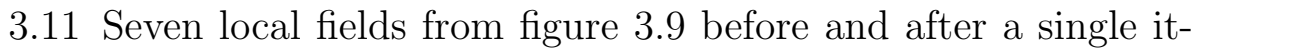
eration step. Each field is rotated by the same angle. . . . . . 76 
3.12 Three-dimensional plot of a configuration of local fields (128 spins with three components) in the low temperature phase before (blue) and after (pink) a single iteration step (the pink configuration is shifted slightly upwards $(+0.01)$ to distinguish it from the blue one). In contrast to figure 3.9 each configuration is one-dimensional and there is no rotation for this lattice at the chosen temperature. . . . . . . . . . . . 76

3.13 First and second component of a single effective cavity field for four different Bethe lattices of 64 spins with $k+1=8$. Different colours represent different temperatures, decreasing from red to green to blue to pink. The plot presents the different possible scenarios: The field is frozen in time (lower left plot and single red or green cross in upper right plot), rotations (ellipses, e.g. lower and upper right plot) or a mixture of rotations and fixed contributions, possibly rotating in a space the size of which is larger than the available $m$ components (pink boxes in upper left plot). . . . . . . . . . . 77

3.14 Unscaled effective component number $m_{\mathrm{eff}}(T)$ for different system sizes. . . . . . . . . . . . . . . . 79

3.15 Scaling plot for $N^{-\mu_{m}}\left(m_{\mathrm{eff}}(T)-c\right)$ against the scaling function

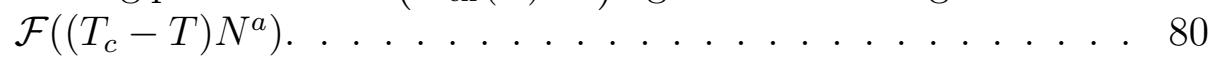

4.1 Plot of $m_{\text {eff }}(T)$ (left axis, + ) and $(\Delta h)^{2}(\times)$ with error bars corresponding to $\operatorname{var}_{\Delta h}$ that indicate the failing of convergence (right axis) for $N=256, k+1=8$ and $m=8$. Replica symmetry is broken slightly below the temperature where $m_{\mathrm{eff}}(T)$ crosses the maximal number of components available, $m$. . . . 84

\begin{tabular}{|ll||l||l|}
\hline 4.2 & Corresponding plot 256 spins with eight components
\end{tabular} on Bethe lattices with connectivity equal to four. . . . . . . . 84

\begin{tabular}{|ll||l||l}
\hline 4.3 & Corresponding plot to & 4.1 & for 96 spins with eight components
\end{tabular} on Bethe lattices with connectivity equal to eight. . . . . . . . 85

\begin{tabular}{|ll||l||l|l}
\hline 4.4 & Corresponding plot to & 4.1 & for spins with four components
\end{tabular} on Bethe lattices with connectivity equal to six. . . . . . . . . 85

\begin{tabular}{|ll||l||l|}
\hline 4.5 & Corresponding 128 spins with three components
\end{tabular} on Bethe lattices with connectivity equal to seven. . . . . . . . 86

4.6 Finite size scaling plot (doubled logarithmic) of the variance

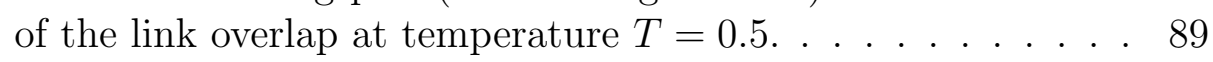


4.7 Possible "phase diagram" of spin glasses for general $m$-component vector spins concerning the question of stable or broken replica symmetry (depending on the number of components as $\frac{1}{m}$ and the dimension of the underlying lattice as $\frac{1}{d}$ ). The line of separation between RSB and RS is motivated in the text. . . . 113

5.1 Finite size scaling plot of the variance of the link overlap at temperature $T=0.6$. See text for explanation. . . . . . . . . 135

6.1 Schematic plot of a vortex for which the spins rotate once anticlockwise on travelling anticlockwise around the centre.

In the low temperature phase, vortices are bound in pairs. . . 146 


\section{List of Tables}

2.1 Critical temperature from the self-consistent effective field ap-

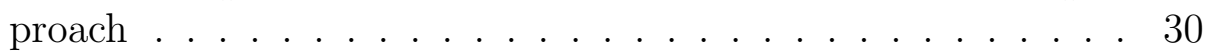

3.1 Results for $\mu_{m}$ for different connectivities $k+1$. . . . . . . 63

3.2 Results for the exponents $\mu_{m}, \nu, x$ and $y$ and the ground state energy per spin and component for different connectivities $k+$ 


\section{Bibliography}

[1] S. F. Edwards and P. W. Anderson. Theory of spin glasses. J. Phys. F, 5:965, 1975.

[2] D. Sherrington and S. Kirkpatrick. Solvable model of a spin-glass. Phys. Rev. Lett., 35:1792, 1975.

[3] J. A. Mydosh. Spin Glasses. An experimental introduction. Taylor \& Francis, London, 1993.

[4] M. Mézard, G. Parisi, and M.A. Virasoro. Spin Glass Theory and Beyond. World Scientific, Singapore, 1987.

[5] K. H. Fischer and J. A. Hertz. Spin Glasses. Cambridge University Press, 1991.

[6] J. R. L. de Almeida and D. J. Thouless. Stability of the sherringtonkirkpatrick solution of a spin glass model. J. Phys. A, 11:983, 1978.

[7] A. J. Bray and M. A. Moore. Replica symmetry and massless modes in the Ising spin glass. J. Phys. C, 12:79-104, 1979.

[8] G. Parisi. Infinite number of order parameters for spin-glasses. Phys. Rev. Lett., 43(23):1754-1757, 1979.

[9] G. Parisi. The order parameter for spin glasses: A function on the interval $0-1$. J. Phys. A, 13:L115, 1980.

[10] G. Parisi. A sequence of approximated solutions to the S-K model for spin glasses. J. Phys. A, 13:L115-L121, 1980.

[11] M. Talagrand. The generalized parisi formula. Comptes Rendus Math., 337(2):111-114, 2003.

[12] C. M. Newman and D. L. Stein. Ordering and broken symmetry in short-ranged spin glasses. J. Phys. Cond. Mat., 15:R1319-R1364, 2003. 
[13] D. S. Fisher and D. A. Huse. Ordered phase of short-range Ising spinglasses. Phys. Rev. Lett., 56(15):1601-1604, 1986.

[14] A. J. Bray and M. A. Moore. Scaling theory of the ordered phase of spin glasses. In L. Van Hemmen and I. Morgenstern, editors, Heidelberg Colloquium on Glassy Dynamics, volume 257 of Lecture Notes in Physics, Heidelberg, 1987. Springer Verlag.

[15] D. S. Fisher and D. A. Huse. Equilibrium behavior of the spin-glass ordered phase. Phys. Rev. B, 38(1):386-411, 1988.

[16] K. Jonason, E. Vincent, J. Hammann, J.-P. Bouchaud, and P. Nordblad. Memory and chaos effects in spin glasses. Phys. Rev. Lett., 81(15):32433246, 1998.

[17] V. Dupuis, E. Vincent, J.-P. Bouchaud, J. Hammann, A. Ito, and H. Aruga Katori. Aging, rejuvenation, and memory effects in Ising and Heisenberg spin glasses. Phys. Rev. B, 64:174204, 2001.

[18] P. E. Jönsson, R. Mathieu, and P. Nordblad. Nonequilibrium dynamics of spin glasses: examination of the ghost domain scenario. Phys. Rev. $B, 70(174402), 2004$.

[19] Florent Krzakala and Federico Ricci-Tersenghi. Aging, memory and rejuvenation: some lessons from simple models. Journal of Physics: Conference Series, 40(1):42, 2006.

[20] M. Mézard and G. Parisi. The bethe lattice spin glass revisited. Eur. Phys. J. B, 20:217-233, 2001.

[21] A. Braunstein, M. Mézard, and R. Zecchina. Survey propagation: an algorithm for satisfiability. Random Structures and Algorithms, 27:201226, 2005.

[22] E. Marinari and G. Parisi. Simulated tempering: A new monte carlo scheme. EPL (Europhysics Letters), 19(6):451, 1992.

[23] H. Nishimori. Statistical Physics of Spin Glasses and Information Processing. Oxford University Press, New York, 2001.

[24] N. Sourlas. Spin glass models as error-correcting codes. Nature, 339:693695, 1989. 
[25] Andreas Martin Lisewski and Olivier Lichtarge. Untangling complex networks: Risk minimization in financial markets through accessible spin glass ground states. Physica A: Statistical Mechanics and its Applications, 389(16):3250 - 3253, 2010.

[26] Jun ichi Maskawa. Multivariate markov chain modeling for stock markets. Physica A: Statistical Mechanics and its Applications, 324(1-2):317 - 322, 2003. Proceedings of the International Econophysics Conference.

[27] Stefano Galluccio, Jean-Philippe Bouchaud, and Marc Potters. Rational decisions, random matrices and spin glasses. Physica A: Statistical and Theoretical Physics, 259(3-4):449 - 456, 1998.

[28] C. De Dominicis, I. Kondor, and T. Temesvári. Beyond the SherringtonKirkpatrick model. In A. P. Young, editor, Spin glasses and random fields, volume 12 of Series on Directions in Condensed Matter Physics, pages 119-160, Singapore, 1998. World Scientific.

[29] M. A. Moore and A. J. Bray. Disappearance of the de Almeida-Thouless line in six dimensions. ArXiv e-prints, February 2011.

[30] J Villain. Two-level systems in a spin-glass model. i. general formalism and two-dimensional model. Journal of Physics C: Solid State Physics, 10(23):4793, 1977.

[31] J Villain. Two-level systems in a spin-glass model: Ii. three-dimensional model and effect of a magnetic field. Journal of Physics C: Solid State Physics, 11(4):745, 1978.

[32] Koji Hukushima and Hikaru Kawamura. Monte carlo simulations of the phase transition of the three-dimensional isotropic heisenberg spin glass. Phys. Rev. B, 72(14):144416, Oct 2005.

[33] Hikaru Kawamura. Chirality scenario of the spin-glass ordering. Journal of the Physical Society of Japan, 79(1):011007, 2010.

[34] L. W. Lee and A. P. Young. Large-scale monte carlo simulations of the isotropic three-dimensional heisenberg spin glass. Phys. Rev. B, 76(2):024405, Jul 2007.

[35] J. H. Pixley and A. P. Young. Large-scale monte carlo simulations of the three-dimensional $x y$ spin glass. Phys. Rev. B, 78(1):014419, Jul 2008. 
[36] L. A. Fernandez, V. Martin-Mayor, S. Perez-Gaviro, A. Tarancon, and A. P. Young. Phase transition in the three dimensional heisenberg spin glass: Finite-size scaling analysis. Phys. Rev. B, 80(2):024422, Jul 2009.

[37] T. H. Berlin and M. Kac. The spherical model of a ferromagnet. Phys. Rev., 86:821, 1952.

[38] J. M. Kosterlitz, D. J. Thouless, and R. C. Jones. Spherical model of a spin-glass. Phys. Rev. Lett., 36:1217, 1976.

[39] M. L. Mehta and Eugen Merzbacher. Random matrices and the statistical theory of energy levels. Physics Today, 22(4):101-103, 1969.

[40] S F Edwards and R C Jones. The eigenvalue spectrum of a large symmetric random matrix. Journal of Physics A: Mathematical and General, 9(10):1595, 1976.

[41] J. R. L. de Almeida, R. C. Jones, J. M. Kosterlitz, and D. J. Thouless. The infinite-ranged spin glass with $m$-component spins. J. Phys. $C$, 11:L871, 1978.

[42] A. J. Bray and M. A. Moore. Replica symmetry and massless modes in spin glasses: Ii. non-ising spins. J. Phys. C, 12:1349, 1979.

[43] J. E. Green, A. J. Bray, and M. A. Moore. Critical behaviour of an $m$-vector spin glass for $m=\infty$. J. Phys. A, 15:2307-2314, 1982.

[44] L. Viana. Infinite-component spin-glass model in the low-temperature phase. J. Phys. A, 21:803-813, 1988.

[45] M. B. Hastings. Ground state and spin-glass phase of the large-n infiniterange spin glass via supersymmetry. J. Stat. Phys., 99:171, 2000.

[46] T. Aspelmeier and M. A. Moore. Generalized Bose-Einstein phase transition in large- $m$ component spin glasses. Phys. Rev. Lett., 92:077201, 2004.

[47] L. W. Lee, A. Dhar, and A. P. Young. Spin glasses in the limit of an infinite number of spin components. Phys. Rev. E, 71:036146, 2005.

[48] A. Braun and T. Aspelmeier. The $m$-component spin glass on a Bethe lattice. Phys. Rev. B, 74(14):144205, 2006.

[49] J M Kosterlitz and D J Thouless. Ordering, metastability and phase transitions in two-dimensional systems. Journal of Physics C: Solid State Physics, 6(7):1181, 1973. 
[50] L. Viana and C. Villarreal. The infinite component EA spin-glass model revisited. J. Phys. A, 26:2873-2881, 1993.

[51] L. W. Lee and A. P. Young. Defect energy of infinite-component vector spin glasses. Phys. Rev. E, 72:036124, 2005.

[52] B. M. Morris, S. G. Colborne, A. J. Bray, M. A. Moore, and J. Canisius. Zero-temperature critical behaviour of vector spin glasses. J. Phys. C, 19(8):1157-1171, 1986.

[53] N. Goldenfeld. Lectures on Phase Transitions and the Renormalization Group. Westview Press, 1992.

[54] T. Aspelmeier, A. Billoire, E. Marinari, and M. A. Moore. Finite size corrections in the Sherrington-Kirkpatrick model. J. Phys. A, 41:324008, 2008.

[55] Giorgio Parisi and Tommaso Rizzo. Phase diagram and large deviations in the free energy of mean-field spin glasses. Phys. Rev. B, 79:134205, 2009 .

[56] G. Parisi and T. Rizzo. Large deviations of the free energy in diluted mean-field spin-glass. J. Phys. A: Math. Gen., 43(4):045001, 2010.

[57] T. Aspelmeier and A. Braun. Sample-to-sample fluctuations and bond chaos in the $m$-component spin glass. Phys. Rev. B, 81(9):094439, 2010.

[58] J.-P. Bouchaud, F. Krzakala, and O. C. Martin. Energy exponents and corrections to scaling in Ising spin glasses. Phys. Rev. B, 68:224404, 2003.

[59] A. Andreanov, F. Barbieri, and O. C. Martin. Large deviations in spinglass ground-state energies. Eur. Phys. J. B, 41(3):365-375, 2004.

[60] S. Boettcher and T. M. Kott. Exact enumeration of ground states in the Sherrington-Kirkpatrick spin glass. Phys. Rev. B, 72:212408, 2005.

[61] B. Derrida. Random-energy model: An exactly solvable model of disordered systems. Phys. Rev. B, 24(5):2613-2626, 1981.

[62] B. Derrida and G. Toulouse. Sample to sample fluctuations in the random energy-model. J. Phys. Lett. (Paris), 46(6):L223-L228, 1985.

[63] J.-P. Bouchaud and M. Mézard. Universality classes for extreme-value statistics. J. Phys. A, 30(23):7997-8015, 1997. 
[64] M. Mézard and G. Parisi. The cavity method at zero temperature. J. Stat. Phys., 111:1, 2003.

[65] Nikos S. Skantzos, Isaac Pérez Castillo, and Jonathan P. L. Hatchett. Cavity approach for real variables on diluted graphs and application to synchronization in small-world lattices. Phys. Rev. E, 72(6):066127, Dec 2005.

[66] A. C. C. Coolen, N. S. Skantzos, I. Pérez Castillo, C. J. Pérez Vicente, J. P. L. Hatchett, B. Wemmenhove, and T. Nikoletopoulos. Finitely connected vector spin systems with random matrix interactions. J. Phys. A: Math. Gen., 38:8289-8317, 2005.

[67] A. Braun. Das $m$-Komponenten Spinglas auf dem Bethe-Gitter. Diploma thesis, Universität Göttingen, 2005.

[68] C. M. Bender and S. A. Orszag. Advanced Mathematical Methods for Scientists and Engineers. Springer Verlag, New York, 1999.

[69] A. J. Bray and M. A. Moore. Metastable states, internal field distributions and magnetic excitations in spin glasses. J. Phys. C, 14:2629, 1981.

[70] A. J. Bray and M. A. Moore. On the eigenvalue spectrum of the susceptibility matrix for random spin systems. J. Phys. C, 15:L765, 1982.

[71] W. H. Press, S. A. Teukolsky, W. T. Vetterling, and B. P. Flannery. Numerical Recipes in $\mathrm{C}++$. Cambridge University Press, Cambridge, 2 edition, 2002.

[72] Y. Y. Goldschmidt and C. De Dominicis. Replica symmetry breaking in the spin-glass model on lattices with finite connectivity: application to graph partitioning. Phys. Rev. B, 41(4):2184-2197, 1990.

[73] A. Crisanti, G. Paladin, H.-J. Sommers, and A. Vulpiani. Replica trick and fluctuations in disordered systems. J. Phys. I France, 2:1325-1332, 1992.

[74] G. Parisi and T. Rizzo. Universality and deviations in disordered systems. Phys. Rev. B, 81:094201, 2010.

[75] T. Aspelmeier and M. A. Moore. Free energy fluctuations in Ising spin glasses. Phys. Rev. Lett., 90(17):177201, 2003. 
[76] T. Aspelmeier. Free-energy fluctuations and chaos in the SherringtonKirkpatrick model. Phys. Rev. Lett., 100(11):117205, 2008.

[77] T. Aspelmeier. An exact relation between free energy fluctuations and bond chaos in the Sherrington-Kirkpatrick model. J. Stat. Mech., page P04018, 2008.

[78] T. Aspelmeier. Bond chaos in the Sherrington-Kirkpatrick model. J. Phys. A, 41:205005, 2008.

[79] G. Parisi, F. Ritort, and F. Slanina. Critical finite-size corrections for the Sherrington-Kirkpatrick spin glass. J. Phys. A, 26:247-259, 1993.

[80] G. Biroli, J.-P. Bouchaud, and M. Potters. Extreme value problems in random matrix theory and other disordered systems. J. Stat. Mech., 2007:P07019, 2007.

[81] C. A. Tracy and H. Widom. The distribution of the largest eigenvalue in the Gaussian ensembles: $\beta=1,2,4$. In J. F. van Diejen and L. Vinet, editors, Calogero-Moser-Sutherland models, number 4 in CRM Series in Mathematical Physics, pages 461-472, New York, 2000. Springer Verlag.

[82] I. Kondor. Parisi's mean-field solution for spin glasses as an analytic continuation in the replica number. J. Phys. A, 16:L127-L131, 1983.

[83] J. Wehr and M. Aizenman. Fluctuations of extensive functions of quenched random couplings. J. Stat. Phys., 60(3-4):287, 1990.

[84] Boettcher, S. Numerical results for spin glass ground states on bethe lattices: Gaussian bonds. Eur. Phys. J. B, 74(3):363-371, 2010.

[85] T. Rizzo. Against chaos in temperature in mean-field spin glasses. $J$. Phys. A, 34(27):5531-5549, 2001.

[86] T. Rizzo and A. Crisanti. Chaos in temperature in the SherringtonKirkpatrick model. Phys. Rev. Lett., 90:137201, 2003.

[87] Giorgio Parisi and Tommaso Rizzo. Chaos in temperature in diluted mean-field spin-glass. Journal of Physics A: Mathematical and Theoretical, 43(23):235003, 2010.

[88] F. Guerra and F. L. Toninelli. The thermodynamic limit in mean field spin glass models. Commun. Math. Phys., 230:71-79, 2002. 
[89] S. Franz, G. Parisi, and M. A. Virasoro. The replica method on and off equilibrium. J. Physique I, 2:1869, 1992.

[90] Martin Weigel and Michel J. P. Gingras. Ground states and defect energies of the two-dimensional $x y$ spin glass from a quasiexact algorithm. Phys. Rev. Lett., 96(9):097206, Mar 2006.

[91] Martin Weigel and Michel J. P. Gingras. Zero-temperature phase of the $x y$ spin glass in two dimensions: Genetic embedded matching heuristic. Phys. Rev. B, 77(10):104437, Mar 2008.

[92] H S Bokil and A P Young. Study of chirality in the two-dimensional xy spin glass. Journal of Physics A: Mathematical and General, 29(5):L89, 1996.

[93] Dao Xuan Viet and Hikaru Kawamura. Monte carlo studies of chiral and spin ordering of the three-dimensional heisenberg spin glass. Phys. Rev. B, 80(6):064418, Aug 2009.

[94] K. Hukushima and H. Kawamura. Chiral-glass transition and replica symmetry breaking of a three-dimensional heisenberg spin glass. Phys. Rev. E, 61(2):R1008-R1011, Feb 2000. 


\section{Danksagung}

Mein Dank gilt in erster Linie Timo Aspelmeier, von dem ich viel lernen durfte und mit dem ich sehr gerne zusammengearbeitet habe.

Ein großer Dank geht an Prof. Dr. Annette Zippelius, für die Möglichkeit, bei ihr zu promovieren und die Unterstützung in all den Jahren.

Ich danke ebenfalls der Deutschen Forschungsgemeinschaft, durch die diese Arbeit unter der Fördernummer AS 136/2-1 finanziert wurde.

Außerdem bedanke ich mich ganz herzlich bei Fr. Schubert, Fr. Glormann, Fr. Mesecke und Fr. Lütge-Hampe. Es war immer gut zu wissen, dass man Sie jederzeit um Hilfe bitten konnte.

Vielen Dank an Martin, Jonathan und Christoph. Ohne euch hätte sicher viel gefehlt!

Vielen Dank auch an all die anderen Kollegen, die mich auf meinem Weg (zum Ende der Arbeit und zum Mittagessen) begleitet haben, wie Stefan F., Garrit, Henning, Christian, Peter, Richard, Timo, Andrea, Alice, Till und Stefan U..

Wie jeder weiß, der eine solch aufwändige Arbeit geschrieben hat, kann diese einen ziemlich vereinnahmen, sowohl zeitlich als auch emotional. Da ist es schön, wenn man Menschen hat, die einem immer wieder zeigen, daß es im Leben doch noch mehr gibt. Für eben diese Menschen sind diese letzte Zeilen.

Ein besonderer Dank geht an meine Familie! Mama, Papa, Tatti und Jochen, ich weiss, daß ihr immer für mich da seid.

Danke Jan-Moritz und Donnie für euer durchgehendes Interesse und die Motivation, tatsächlich zu einem Ende zu kommen.

Danke Claudi, für alles. 



\section{Curriculum Vitae}

Axel Braun

Huttenstraße 3

34119 Kassel

E-mail: braun-axel@gmx.de

\section{Persönliche Daten}

Geburtsdatum: $\quad 25.01 .1980$

Geburtsort: Kassel, Hessen

Staatsangehörigkeit: deutsch

Familienstand: ledig, ein Kind (Luis, geb. 31.3.2009)

\section{Wissenschaftlicher Werdegang}

1999 Abitur am Georg-Christoph-Lichtenberg Gymnasium, Kassel

2000 Beginn des Studiums der Physik an der Georg-August-Universität Göttingen

2002 Vordiplom an der Fakultät für Physik der Universität Göttingen

2005 Diplom, Diplomarbeit am Institut für Theoretische Physik der Universität Göttingen mit dem Titel "Das $m$-Komponenten Spinglas auf dem Bethegitter"

2006 Beginn der Promotion am Institut für Theoretische Physik der Universität Göttingen

\section{Publikationen:}

- A. Braun and T. Aspelmeier. The m-component spin glass on a Bethe lattice. Phys. Rev. B, 74(14):144205, 2006

- T. Aspelmeier and A. Braun. Sample-to-sample fluctuations and bond chaos in the m-component spin glass. Phys. Rev. B, 81(9):094439, 2010

Göttingen, den 25.5.2011 